
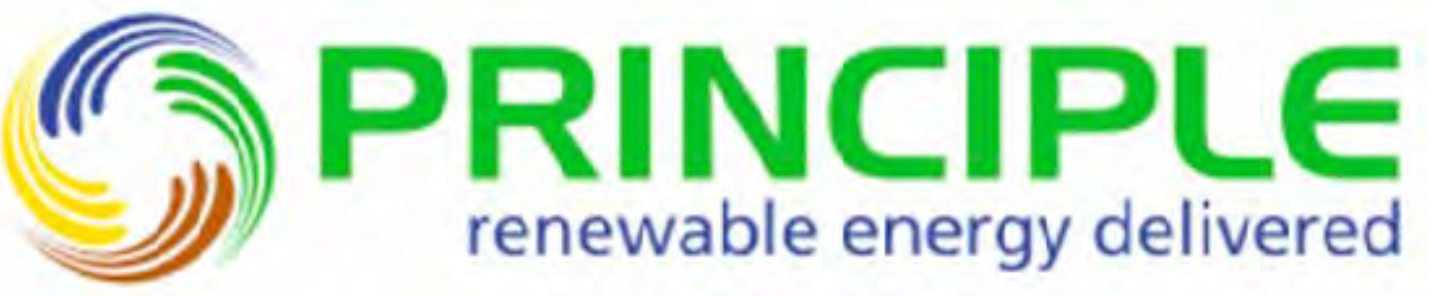

DOE Grant to Support the Development of Advanced Water Power Technologies

\title{
WindWaveFloat (WWF): \\ Final Scientific Report
}

DOE Grant DE-EE0002651

Recipient:

Principle Power, Inc. (PPI)

Consortium Members: National Renewable Energy Laboratory (NREL) Marine Innovation \& Technology (MI\&T)

Project Contact(s): $\quad$ Alla Weinstein, CEO PPI Karen Ho, Controller PPI

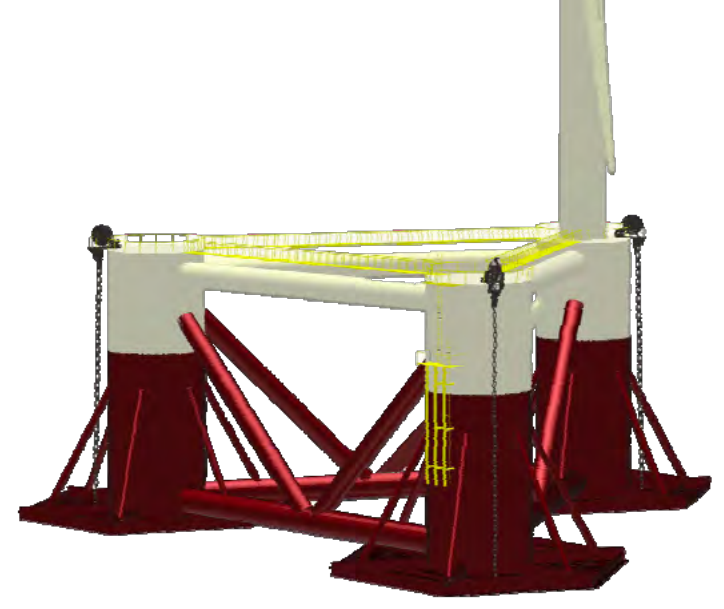




\section{Table of Contents}

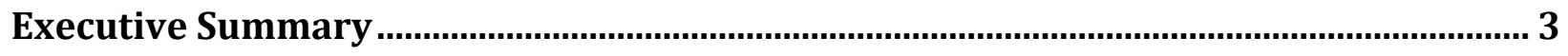

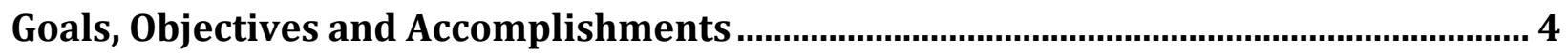

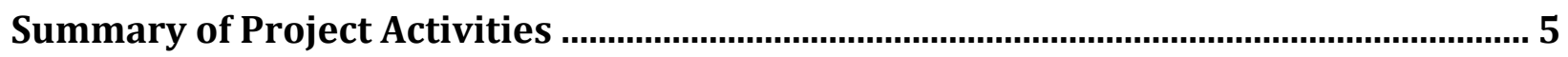

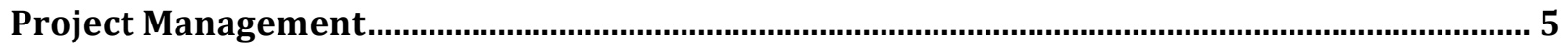

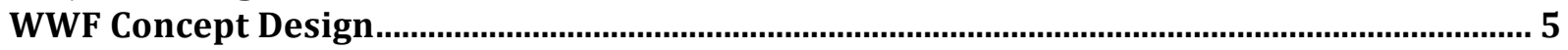

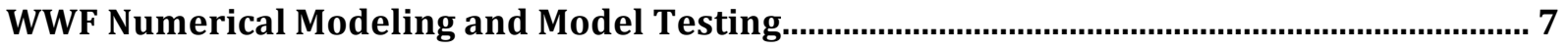

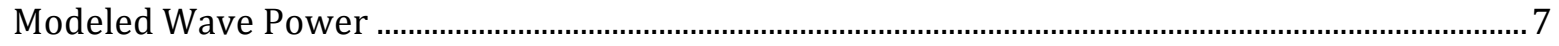

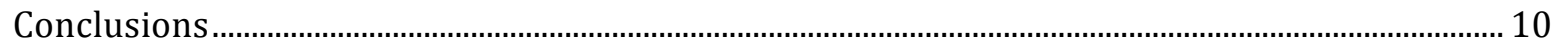

Permitting and Grid Integration.........................................................................................10

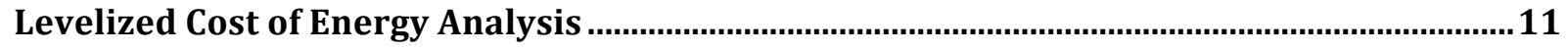

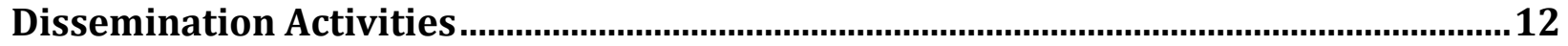




\section{Executive Summary}

The main challenge for offshore floating renewable energy devices remains to build a structure capable of withstanding the highly energetic ocean environment in such a way that costs are competitive in global energy markets. Due to technological similarities to onshore wind energy and continually growing demand for better wind resources close to load, in certain parts of the world the offshore wind industry is developing quickly. Principle Power, Inc.'s (PPI) WindFloat is a floating foundation for multi- megawatt wind turbines. The WindFloat is a three-column semi-submersible platform designed to harvest wind energy in water depths of 45 meters and deeper, where fixed offshore turbines cease being economical. The present WindFloat deploys an industry standard horizontal-axis wind turbine at the top of a tower, on one of the three columns and is suitable to accept any other wind turbines configurations as maybe chosen by an end user. In this configuration, the WindFloat has enough deck space for additional equipment.

Principle Power Inc. and National Renewable Energy Lab (NREL) have completed a contract to assess the technical and economic feasibility of integrating wave energy converters into the WindFloat, resulting in a new concept called the WindWaveFloat (WWF) under the DOE Grant DE-EE0002651.

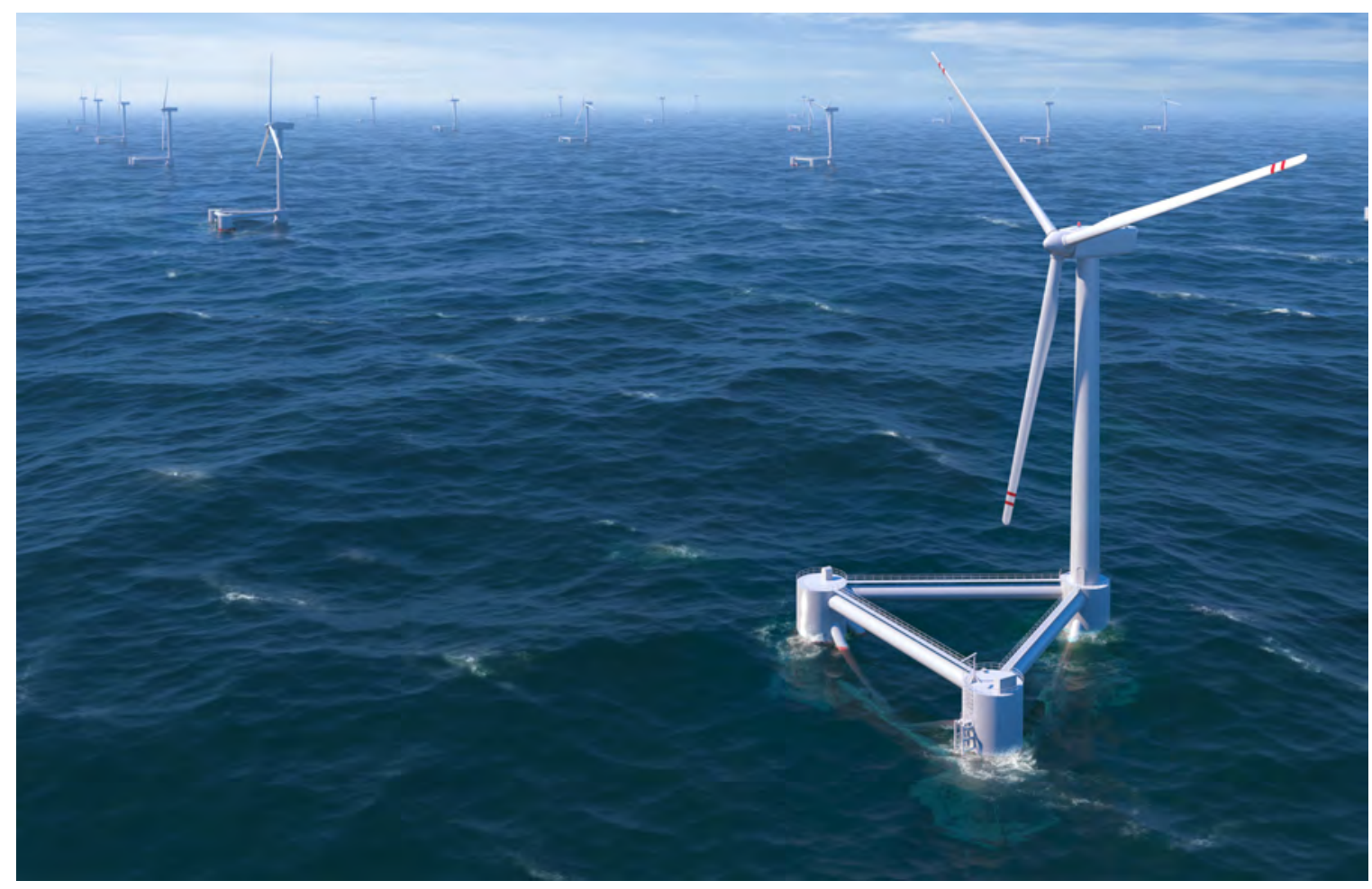

Figure 1: Artistic rendering of a WindFloat wind farm.

The concentration of several devices on one platform could offer a potential for both economic and operational advantages. Wind and wave energy converters can share the electrical cable and power transfer equipment to transport the electricity to shore. Access 
to multiple generation devices could be simplified, resulting in cost saving at the operational level. Overall capital costs may also be reduced, provided that the design of the foundation can be adapted to multiple devices with minimum modifications. Finally, the WindWaveFloat confers the ability to increase energy production from individual floating support structures, potentially leading to a reduction in levelized energy costs, an increase in the overall capacity factor, and greater stability of the electrical power delivered to the grid.

The research conducted under this grant investigated the integration of several wave energy device types into the WindFloat platform. Several of the resulting system designs demonstrated technical feasibility, but the size and design constraints of the wave energy converters (technical and economic) make the WindWaveFloat concept economically unfeasible at this time. Not enough additional generation could be produced to make the additional expense associated with wave energy conversion integration into the WindFloat worthwhile.

\section{Goals, Objectives and Accomplishments}

The goal of the project was to assess technical and economic viability of integrating a number of wave energy PTO mechanisms with the floating support structure in order to share infrastructure and maximize power output, potentially leading to a reduction in the levelized cost of energy (LCOE). Validated numerical models, wave tank testing results, and fabrication specifications developed for PPI's proprietary WindFloat were used as the basis for WWF development. The ultimate LCOE and cost/benefit analysis were to be performed on a commercially sized WWF offshore farm of 150MW capacity, producing energy from offshore wind and waves.

The project included engineering studies, numerical model development, validation of numerical tools through scaled physical models and wave tank testing, performance verification, cost/benefit analysis, and optimization studies to increase energy production. The project was concluded with a technical and economic feasibility analysis of the WWF device, final design configuration, projected LCOE calculations, and follow-on steps towards commercial project development at a specified site offshore Tillamook County, Oregon.

The use of structure hybrid, wind/wave combined system, leads to a number of benefits, including reduction of environmental impact due to shared mooring, and more flexible siting in water depths greater than 150-250 feet. These water depths have previously been considered a maximum for wave energy devices because of the significantly increased cost of mooring in deeper waters. In addition wave and offshore wind resources are intermittent, a disadvantage common to many renewable resources. It was anticipated that a combined wind/wave system could offer a potential to deliver a more consistent and valuable energy product; for example, wave energy conversion continues even in storm conditions, when wind turbines may be shut down. 
The WaveWindFloat project therefore had two main objectives:

- Objective I: to confirm the technical and economic feasibility of an innovative wave-wind floating support structure, the WWF, through the incorporation of a number of wave and wind energy PTO mechanisms.

- Objective II: to assess the LCOE of the WWF as compared with the WindFloat in a commercial scale.

In response to these objectives, several wave energy PTOs were identified and their integration into the floating support structure investigated. A series of numerical models were developed to understand the aero/hydrodynamics of a system that would be subjected to both wind and wave loads. Scale models were developed, built and tested at the wave tank of UC Berkeley, California. The permitting process that would be required for the deployment of a hybrid wind/wave system in United States' waters was investigated.

Data derived from the project enabled project proponents to conclude that the integration of wave energy devices into a stable floating platform designed primarily for the production of wind energy is technically feasible and none of the tested PTOs had impacts on the motions of the WindFloat. However, though technically feasible and possible, a wind and wave hybrid system, did not result in a reduction of LCOE. This stems from a relatively low amount of energy that can be generated by wave energy PTOs (maximum available approximately $250 \mathrm{~kW}$ per each PTO), in comparison to the anticipated costs associated with their development and integration. A top-level assessment of the resulting LCOE confirmed that the WWF, unfortunately, does not reduce the LCOE, as the added energy generation is hindered by an increase in CAPX and OPX.

\section{Summary of Project Activities}

\section{Project Management}

Principle Power performed overall project management, including planning, administrative activities and coordination with and quarterly reporting to the Department of Energy (DOE).

\section{WWF Concept Design}

The project was framed around understanding the technical and economic impacts of adding additional energy generation to a WindFloat, since mooring, electrical infrastructure and other elements of the deployment would be shared.

It was originally thought that incorporating existing PTO concepts by partnering with wave energy specific technology companies would provide the quickest, most effective means to select viable technologies. This lead to the development and commissioning of the wave 
energy PTO State-of-the-Art report, a global survey of wave energy technology companies, products and product technical readiness. The compiled report is included as an appendix.

However, during the development of the report, it became clear that commercial interests, rather than technological advancements, were dominating initial discussions. Multiple companies are working on similar concepts, and each of these companies, with a vested interest in promoting its own technology(ies), was more focused on intellectual property protection than on the incorporation of their PTOs. It was therefore decided to study generic types of wave energy PTOs and focus on the technical integration at this stage of the work.

Primarily, three types of PTO's were studied within the scope of this work. Each was integrated independently into a base case WindFloat, designed to support the generic NREL $5 \mathrm{MW}$ wind turbine. Each wave energy PTO represents a different mechanism and was designed to reflect current developments in the industry. The different technologies primarily investigated were:

Oscillating Water Column. In an oscillating water column (OWC), water enters through a subsurface opening into a chamber that contains air. The wave action causes the captured water column to rise and fall like a piston, compressing and decompressing the air. This air is then channeled through an opening connected to a bi-directional turbine. In this case, the chamber was built externally around the two columns not supporting the turbine.

Single Point Energy absorber. A point absorber is a floating system that absorbs energy from all directions through its movements at the water surface. A point absorber is usually designed to resonate so that its harnessed power is maximized. In this generic study, we looked at a sphere placed in the middle of the WindFloat triangle. The sphere was chosen because it responds well in heave, with very little pitch motion.

Flaps or Oscillating Wave Surge Converter (OWSC). The OWSC comprises a nearsurface collecting system mounted on a pivoting arm installed on the seabed. The arm oscillates like an inverted pendulum in response to the surrounding waves. In this case the plates were mounted on the structure main beams, outside of the water. This presented the advantageous ability to completely remove the flaps from wave actions during large storms, as the beams are designed to be dry and the flaps should be able to be locked in a horizontal position. 


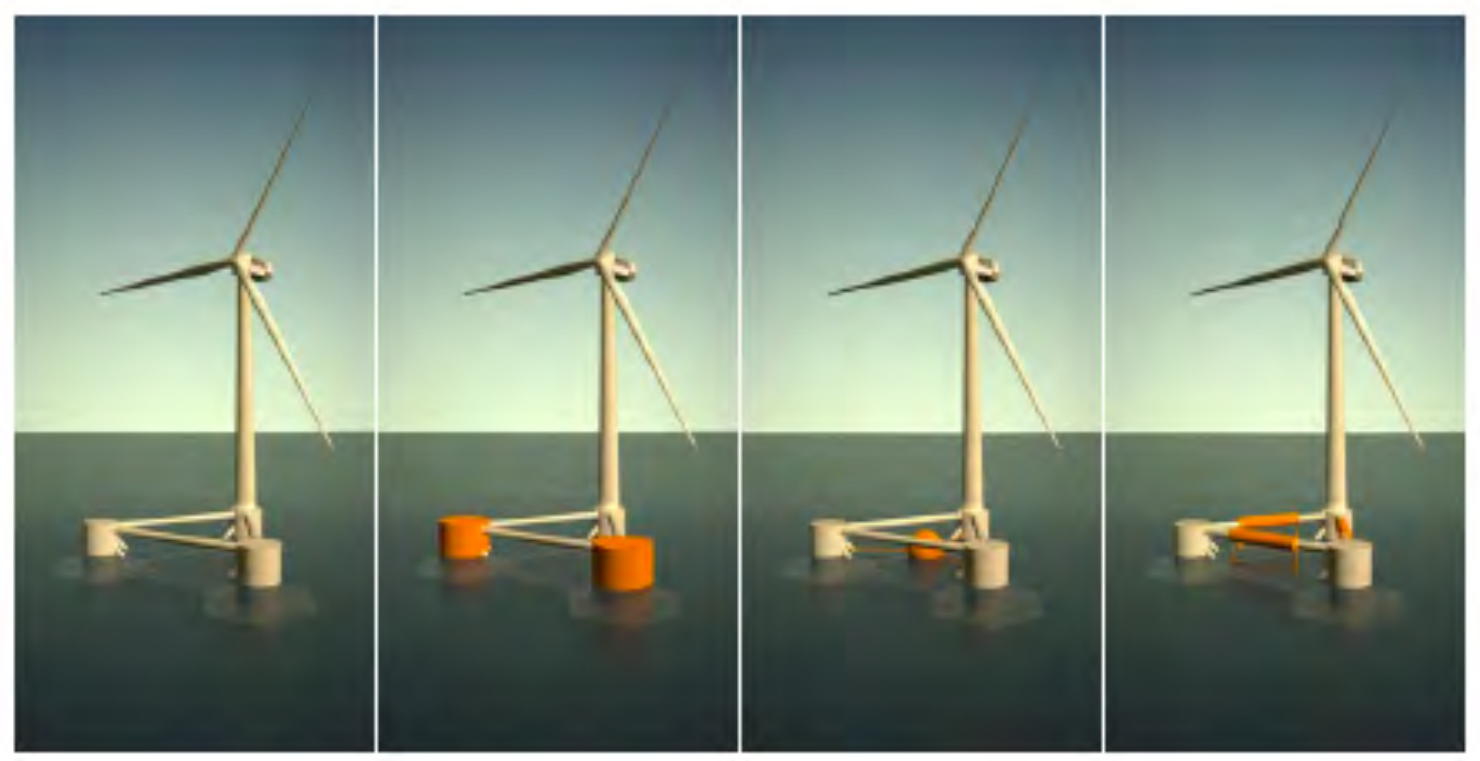

Figure 2: The base case WindFloat; oscillating water column, single point absorber and oscillating plates.

\section{WWF Numerical Modeling and Model Testing}

A report was produced that summarizes the theory and results behind the modeling that was performed to incorporate Wave Energy Converters (WEC) into the WindFloat. Models were developed using the diffraction-radiation code WAMIT and the time domain code ORCAFLEX. In this report, the modeling is first described, then the motion Response Amplitude Operators (RAOs) are computed for each device analyzed. From these motion responses, the theoretical power available was calculated and then confirmed through wave tank model testing. The forces on each device are dependent on the interference between the device and the hull, the mooring, and the non-linear effects, which are challenging to model. Therefore, these forces were approximated using a Morrison-type formulation. The empirical values for drag coefficients, damping and stiffnesses in this report were based on our "best engineering judgment" and can be seen as a "first pass", used to validate that the numerical models were working.

For full discussion of the numerical modeling, anticipated power curves and model testing activities funded under this grant, please refer to the appendices.

\section{Modeled Wave Power}

In the framework of the WindWaveFloat project, a generic 5MW WindFloat platform was used as the structure supporting different wave energy devices. For each of the different generic designs of the WindWaveFloat, numerical and experimental results of the systems were presented in regular wave conditions. In order to study the performance of these WindWaveFloat concepts, the primary objective of this work was to validate the numerical models created both in OrcaFlex and WAMIT (depending on the WEC) using experimental results obtained in the wave tank at model scale. 
After the numerical models were validated, the impact of the WECs on the WindFloat platform motions, and the conversion of wave energy into mechanical energy (or pneumatic energy in the case of the OWC), were evaluated for several stiffness and damping coefficients, modeling potential PTO characteristics in each case. The capture width was also computed and the optimal operating point was deduced by varying the PTO stiffness and damping coefficients for each of the generic WECs.

Finally, a power and/or capture width matrix was derived at the optimal PTO operating point to estimate the average power output that could be harnessed in different regular waves. Discrepancies between numerical models and experimental results can occur because of the presence of non-linear effects. It was important to understand these effects, often linked to the quadratic damping of the system, in order to calibrate the numerical models and accurately estimate the performance of each of the devices.

\section{Oscillating Water Column}

A model was developed to incorporate the specificities of the WindFloat platform in the calculation of the power converted by the turbine. The current numerical model neglects non-linear loads on the platform, as well as turbine damping on the internal water free surface.

- Maximum power of about $139 \mathrm{~kW}$ of average mechanical power would be harnessed with a regular wave height of $2 \mathrm{~m}$.

- The optimal point occurs for a wave of $5 \mathrm{~s}$, a stiffness coefficient of $200 \mathrm{kN} / \mathrm{s}$ and damping coefficient of 200 tonnes/s.

The pneumatic efficiency of the turbine was approximated with a modeled function of flow ratio, which is meant to represent the Wells turbine. This model shows that turbine characteristics are essential in the smoothing and optimization of the power output. Different turbine parameters could be tested to optimize the design. Losses in the turbine rotor and generator were also neglected. The PTO stiffness and damping coefficients were varied for different wave periods (at a given wave slope, thus at a given wave amplitude) to spot the optimal wave power extraction point, which corresponds to the wave and PTO characteristics generating as much mechanical power as possible and as efficiently as possible.

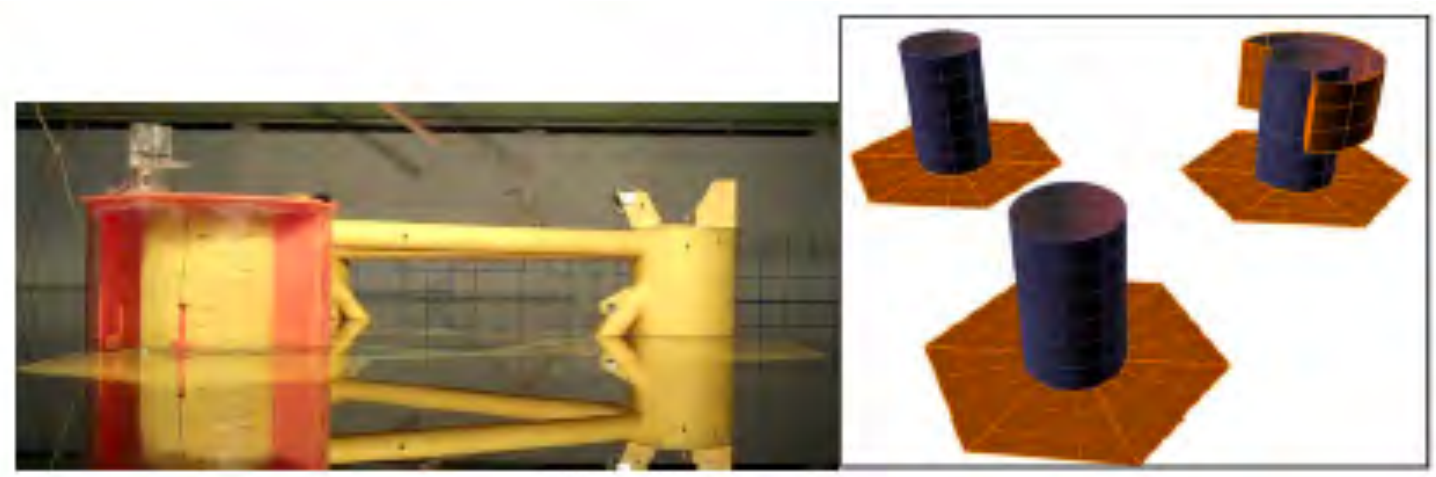

Figure 3: Oscillating water column in wave tank and modeled. 


\section{Single Point Absorber}

- The maximum power of about $50 \mathrm{~kW} / \mathrm{m} 2$ is obtained for a damping of $200 \mathrm{kN.s} / \mathrm{m}$ at a $3 \mathrm{~s}$ wave period, and for a damping of $1000 \mathrm{kN} . \mathrm{s} / \mathrm{m}$ at a $5 \mathrm{~s}$ wave period.

- The surge period of resonance at $3 \mathrm{~s}$ impacts the average power significantly for smaller wave amplitudes.

- The higher the wave amplitude is, the more the heave resonance period of $4-5 \mathrm{~s}$ comes into play.

This can also be due to the following effect: the stiffness of the lines decreases when the pitch motion amplitude increases (for higher wave amplitudes), and thus the resonance period increases.

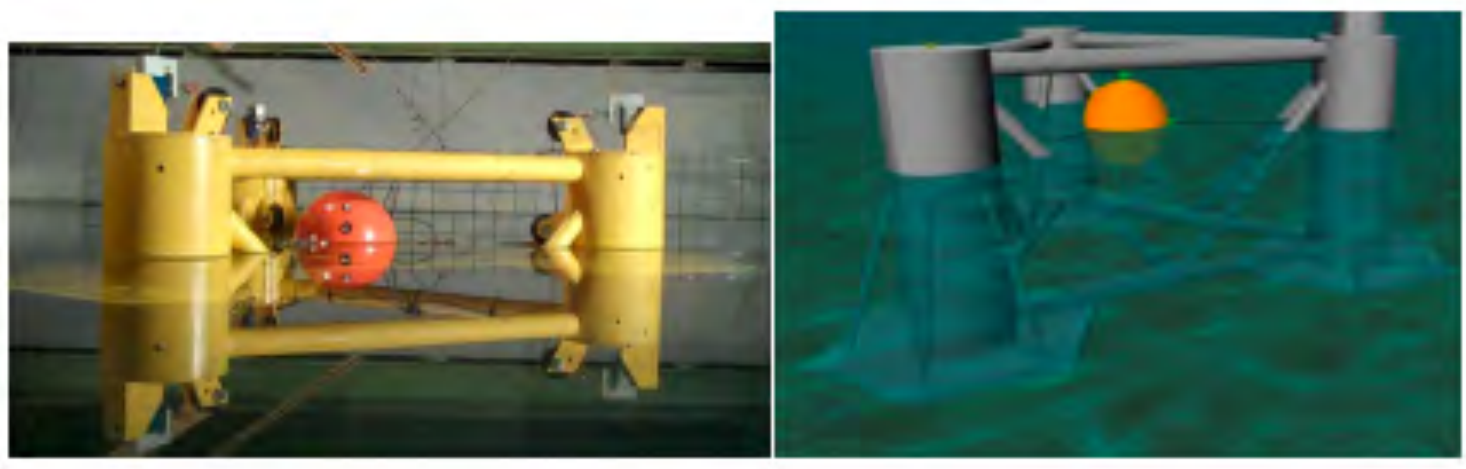

Figure 4: Single point absorber in wave tank and modeled.

\section{Oscillating Plates}

- The maximum power of about $150 \mathrm{~kW} / \mathrm{m} 2$ is always obtained at a wave period of 5$6 \mathrm{~s}$, which corresponds to the flap pitch resonance period.

- The downwave flap, directly perpendicular to the incoming waves, has power performances usually higher than the upwave flaps.

- The higher the wave amplitude is, the more the heave resonance period of $6 \mathrm{~s}$ comes into play.

This can also be due to the following effect: the stiffness of the lines decreases when the pitch motion amplitude increases (for higher wave amplitudes), and thus the resonance period increases. 


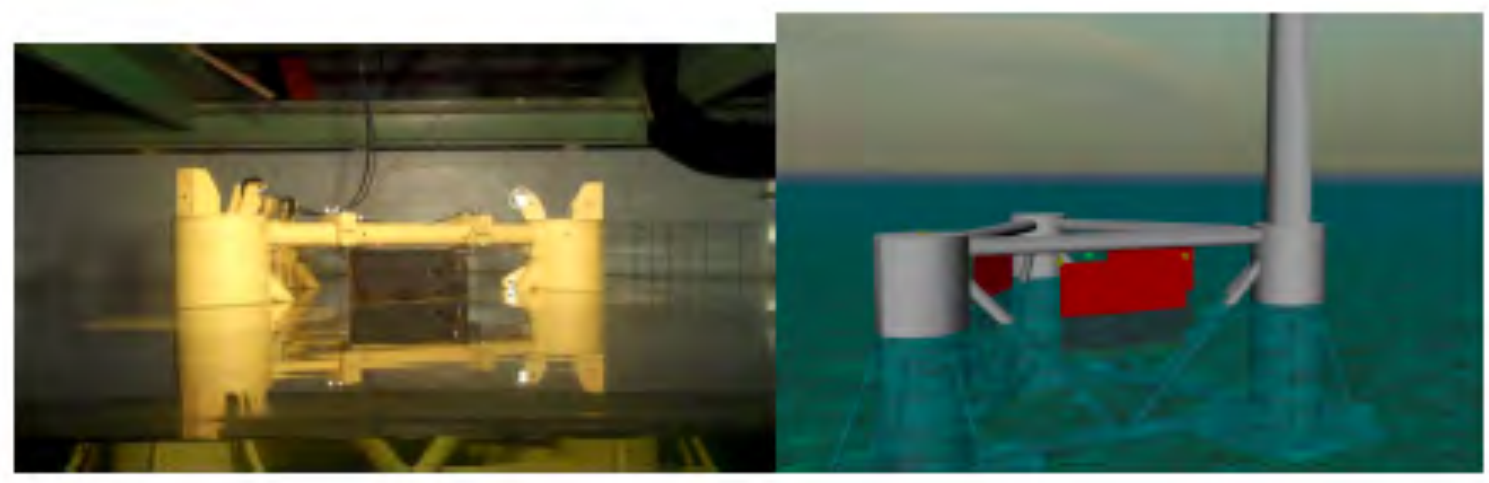

Figure 5: Oscillating plates in wave tank and modeled.

\section{Conclusions}

The different modeled designs only minimally affected the WindFloat platform motion. Oscillating plates had the most significant impact by increasing the surge motion amplitude of the WindFloat platform. Structurally, the effect of the loading of the wave device on the WindFloat hull would still need to be investigated.

Yet calculations show that a maximum average power per wave amplitude squared of only $150 \mathrm{~kW} / \mathrm{m} 2$ would be potentially harnessed in these configurations. These performances occur with regular sinusoidal incoming waves at a zero degree heading, and might decrease substantially with irregular incoming waves. These performances are most likely lower in reality, since shielding effects, radiation effects, and hydrodynamic interferences are not modeled here. Moreover, losses in the PTO systems were not taken into account.

These results led the investigatory team to conclude that the integration of wave energy conversion systems into the WindFloat would not result in an abundantly high addition to the overall energy production from the WindFloat, and, hence economic benefit relative to the WindFloat itself, and that the WindWaveFloat was not an economically viable concept.

\section{Permitting and Grid Integration}

At the time of the report's writing, there was no specialized regulatory path defined for the development of hybrid wind/wave energy system projects in federal waters. While the agencies overseeing the development of these resources appeared to be willing partners, and were reportedly working together to develop a more specialized process, the current prescribed path is convoluted and inefficient. In essence, the process as it stands is an amalgamation of the requirements defined for the exploitation of both wind and wave resources in the ocean.

Any changes that occur to the current set of regulations will be subject to process that, one way or the other, will allow for the public to contribute to the discussion. In the case of prospective changes to the jurisdictions of the agencies, an unlikely outcome, legislated changes would be required. For changes in the way the agencies' existing authorities are administered a rulemaking would be required; this has public comment periods built into the process. A fuller discussion of the potential for, and process required, to create a 
specialized regulatory system for hybrid wind/wave energy devices is beyond the scope of this paper.

At the same time, no commercial projects have yet been proposed, and the technologies are still relatively immature. Hopefully, in this case, the regulatory environment for these technologies will be developed in such a way as to encourage commercial activity.

Over the course of the project, discussions were conducted with Tillamook PUD, the utility at the proposed project location. Tillamook PUD has plans to build a new substation and transmission lines with approximately $200 \mathrm{MW}$ of capacity near Oceanside, OR, near the proposed project site. It was determined that, should a project be developed offshore the area, this would most likely be the preferred point for interconnection to the grid. Accordingly, the interconnection would follow the established protocols for the introduction of new generation onto the Northwest grid at any location. These protocols include the feasibility, system impact and facility studies typically required by the grid operator, NEPA and other regulations. Because the substation and line would be new, there were no anticipated problems associated with interconnecting at the site.

\section{Levelized Cost of Energy Analysis}

To understand the cost of energy impact of the incorporation of wave energy conversion into the floating wind structure, a cost assessment was first required for the WindFloat itself. Having a baseline costs for the WindFloat, allows determination of the relative costs of wind and wave generation as compared to the baseline costs, leading to the determination of the viability of most economic configuration. To establish the baseline costs, the WindFloat's costs were calculated against the conventional, monopile foundations for deep or intermediate water depth offshore wind installations. To achieve this, a cost assessment for the WindFloat Concept and for a conventional substructure was undertaken for two characteristic $40 \mathrm{~m}$ to $50 \mathrm{~m}$ water depth sites, based on a $5 \mathrm{MW}$ WTG. The assessment was commissioned to GL-GH to ensure an independent costs analysis based on actual projects experience.

Table 1: Methodology used in WindFloat LCOE report.

Required capital expenditures (CapEx) and Cost of Energy

of conventional offshore wind and WindFloat concepts

were studied for:

1. a near shore site

2. a far shore site,

in combination with:

1. an optimistic marine equipment day rate scenario

2. an pessimistic marine equipment day rate

scenario,

and in combination with:

1. lower boundary steel prices

2. upper boundary steel prices. 
The conclusion of the report is that the CapEx for the WindFloat concept is in most cases similar to that of a conventional seabed mounted structure. The tendency for near shore is similar to far shore sites and the same applies to the procurement costs for the WTG.

The WindFloat concept clearly shows the benefits of limited marine spread costs, but the material and manufacturing costs of the hull have a noticeable effect on the economic viability of the concept. WindFloat can be regarded as an economically viable competitor against conventional concepts at sites with water depths ranging from 40 to $50 \mathrm{~m}$.

Depending on choice regarding life-extension and eventual decommissioning strategy, additional benefits may be realized for the WindFloat by extending the lifetime of the unit. It must be noted that these conclusions strongly depend on the assumptions with respect to steel price and the day rate of the marine equipment.

The results from the study were encouraging for the WindFloat, particularly because of technical and cost related improvements that can be incorporated into future designs. However, the disappointing results for energy generation from wave power modeling tasks combined with management's understanding of the costs associated wave energy conversion systems, indicated that addition of wave energy conversion PTOs will not improve the LCOE, but could actually make it worse due to increased CAPEx and OPEx. The investigatory team concluded that the WindWaveFloat was not an economically viable concept.

\section{Dissemination Activities}

Dissemination activities for the project included journal submissions and paper presentations at OMAE and EWTEC; these papers are included in the Appendix. Preliminary results have also been presented at numerous conferences, including those hosted by EnergyOcean, the Oregon Wave Energy Trust, AWEA and at the DOE Peer Review. 
Appendix A: Wave Energy PTO Systems State of the Art Report 


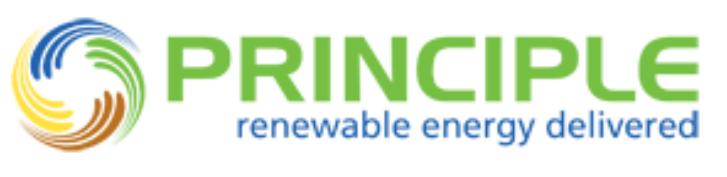

DOE Grant to Support the Development of Advanced Water Power Technologies

\section{WindWaveFloat (WWF)}

\section{WWF CONCEPT DESIGN DELIVERABLE \#1 \\ Wave-Energy PTO Systems \\ State-of-the-Art Report}

\section{$\mathbf{1}^{\text {st }}$ Quarter 2010}




\section{TABLE OF CONTENTS}

I. SCOPE OF THE REPORT.

II. LISTING OF WORLWIDE WAVE-ENERGY TECHNOLOGIES........................................4

III. CHARACTERISTICS OF THESE TECHNOLOGIES......................................................5

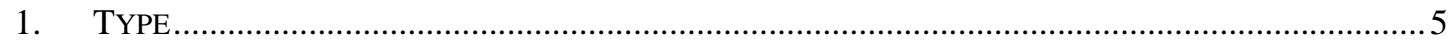

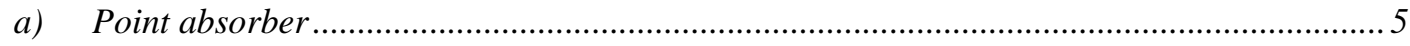

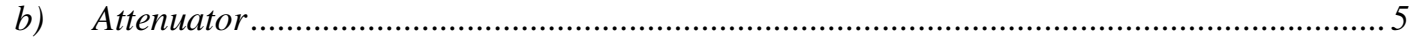

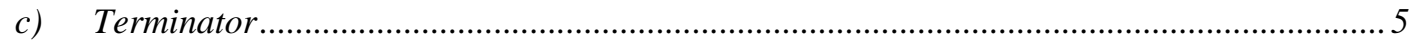

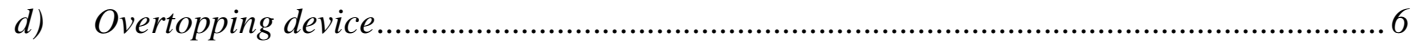

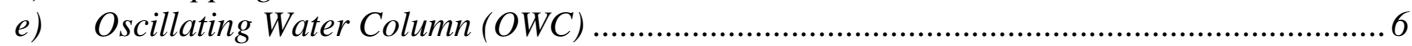

f) Oscillating Wave Surge Converter (OWSC) or Pitching/Surging/Heaving/Sway (PSHS) device.

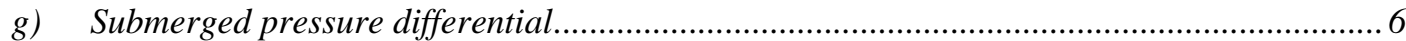

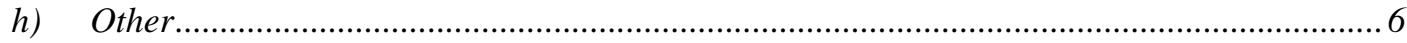

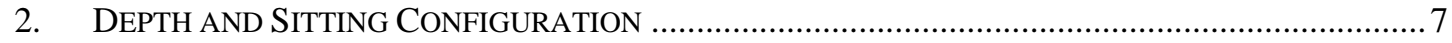

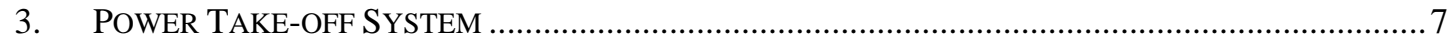

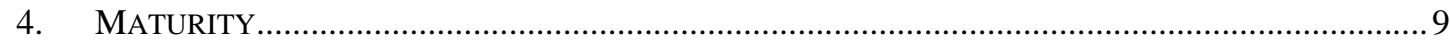

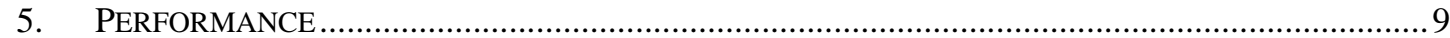

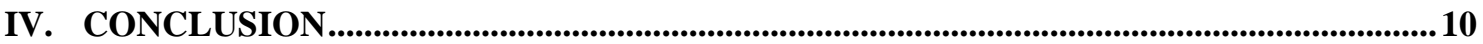

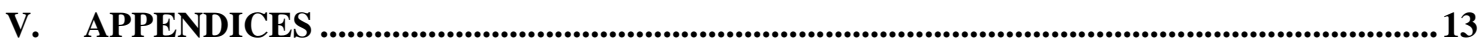

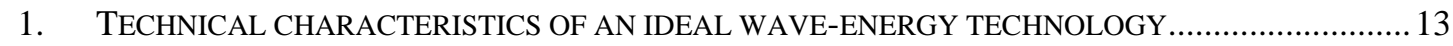

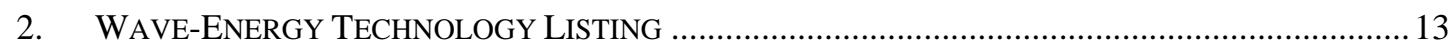




\section{SCOPE OF THE REPORT}

The WWF project is part of the Department of Energy (DOE) Grant to Support the Development of Advanced Water Power Technologies. The goal of the proposed project is to assess feasibility of integrating wave-energy conversion power take-off (PTO) mechanisms with a floating offshore wind support structure - the WindFloat - in order to a) maximize power output, b) share infrastructure, and c) reduce levelized energy cost as compared to the use of the floating support structure only for offshore wind.

This report is the first deliverable of the WWF project. It performs an overview and assessment of the available PTOs worldwide to identify the state of the art. Figure 1 presents the scope of this report within the framework of the approach that leads to the determination of a WWF design concept. The assessment of available wave-energy power take-off systems represents the first step in that determination (red dotted circle).

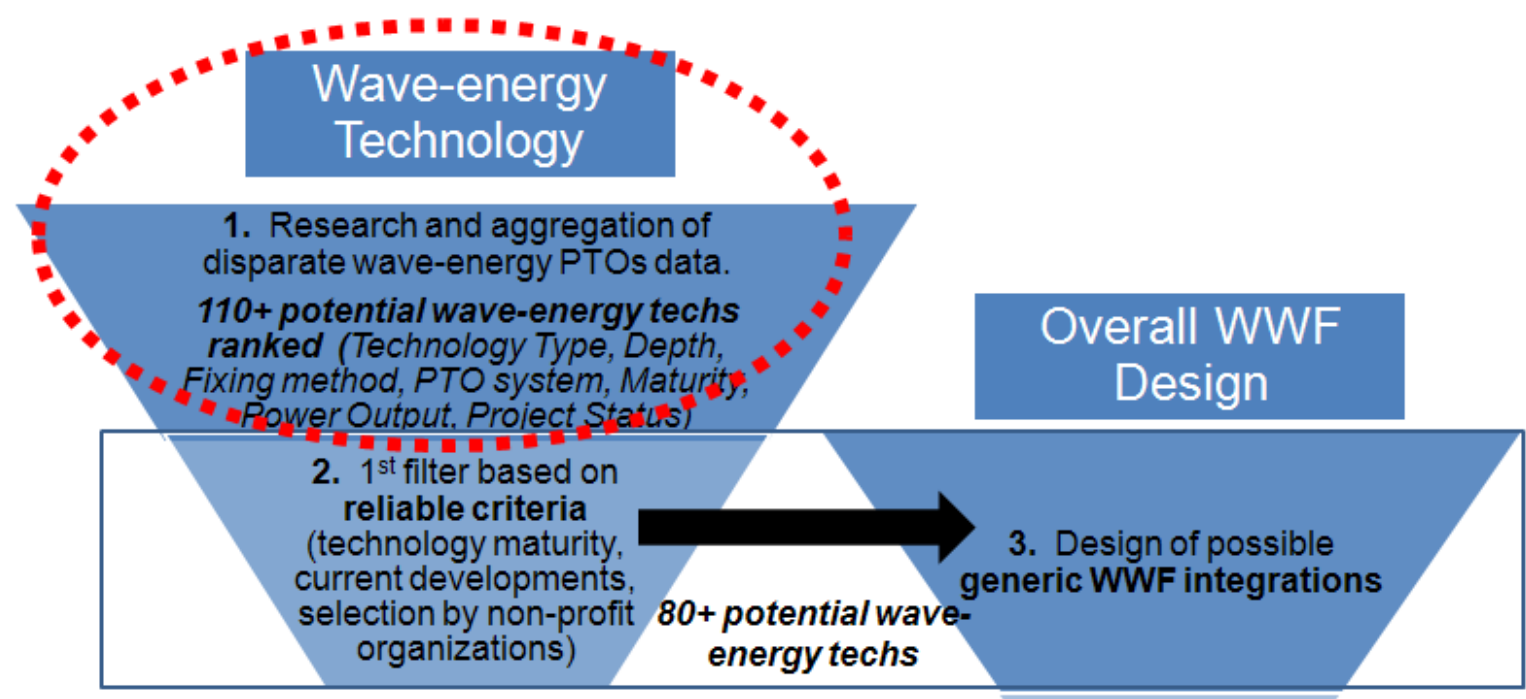

$$
\begin{gathered}
\text { 4. } 2^{\text {nd }} \text { filter based on } \\
\text { WWF suitability, } \\
\text { using feasible generic } \\
\text { integrations. } \\
\text { 20+ wave-energy techs applicable to } \\
\text { each generic design }
\end{gathered}
$$

\section{Figure 1: Scope of the report in the WWF concept design determination process}

The first section of the report presents a complete listing of existing wave-energy technologies on the market. The next sections outline the current status of the wave-energy industry by providing a description and assessment of different wave-energy technology characteristics. The last section concludes on the current status of ocean wave-energy conversion. 


\section{LISTING OF WORLWIDE WAVE-ENERGY TECHNOLOGIES}

Firstly, an exhaustive inventory of existing wave-energy technologies is realized based on the following websites and reports:

- Ocean Energy: Global Technology Development Status prepared by Powertech Labs Inc. for the IEA-OES in March $2009^{1}$.

- $\quad$ Review and analysis of ocean energy systems development and supporting policies, a report by AEA Energy \& Environment on the behalf of Sustainable Energy Ireland for the IEA's Implementing Agreement on Ocean Energy Systems, 28th June $2006^{2}$.

- Ocean Energy, State of the Art, November 2009, prepared by the European Ocean Energy Association ${ }^{3}$.

- Marine Renewable (Wave and Tidal) Opportunity Review ${ }^{4}$, by Scottish Enterprise, December 2005.

- The International Energy Agency Implementing Agreement on Ocean Energy Systems, annual report $2008^{5}$ and 2009.

- $\quad$ The European Marine Energy Centre Ltd Wave Developers .

- Marine and Hydrokinetic Technology Listings from the US Department of Energy ${ }^{7}$.

- Ocean Wave Energy - PESWiki ${ }^{8}$.

- Technology/Company website.

Eventually, more than $\mathbf{1 1 0}$ wave-energy technologies appear in this exhaustive listing (see the Wave-Energy Technology Listing, page 13). In order to compare these wave-energy technologies and understand the status of the industry, the following characteristics are gathered and analyzed for each technology appearing in the listing:

- Technology Type.

- Depth and Sitting configuration

- Power Take-off System.

- Technology Maturity.

- Performance.

These different characteristics are then detailed in each subsection.

\footnotetext{
${ }_{1}^{1}$ IEA-OES Document No. T0104 http://www.iea-oceans.org/_fich/6/ANNEX_1_Doc_T0104.pdf

${ }^{2}$ http://www.iea-oceans.org/_fich/6/Review_Policies_on_OES_2.pdf

${ }^{3}$ Report prepared by Ana Brito e Melo, WAVE ENERGY CENTRE

${ }^{4} \mathrm{http}: / /$ www.oceanrenewable.com/wp-content/uploads/2007/03/oregreport.pdf

${ }^{5}$ http://www.iea-oceans.org/_fich/6/Annual_Report_2008_(1).pdf

${ }^{6}$ http://www.emec.org.uk/wave_energy_developers.asp

${ }^{7}$ http://www1.eere.energy.gov/windandhydro/hydrokinetic/listings.aspx?type=Tech

${ }^{8}$ http://peswiki.com/index.php/Directory:Ocean_Wave_Energy
} 


\section{CHARACTERISTICS OF THESE TECHNOLOGIES}

A wave-energy technology aims at converting the motion of ocean waves into electricity. The process comprises three considerations: wave-energy extraction (converting the wave energy into mechanical energy), its conversion (transforming that mechanical energy into electricity) and the transmission of this electricity from the site to the grid.

Wave-energy converters fall into two main categories:

- Most of the wave-energy technologies use a system that interacts and moves with the wave, and a system that resists this motion to create forces that are then converted to electricity through a power take-off system. This type of device is sometimes called a reacting device.

- Other technologies directly use the motion of the wave to push either water or air through the power take-off system (that is usually a turbine in this case).

These two categories are often undifferentiated when wave-energy technologies are classified by type. The next paragraph summarizes the different types of technologies that exist and are currently used to harness wave power.

1. Type

The most comprehensive classification ${ }^{9}$ of wave-energy technology types is as follows:

\section{a) Point absorber}

A point absorber is a floating system that absorbs energy in all direction through its movements at the water surface. A point absorber is usually designed to resonate so that its harnessed power is maximized.

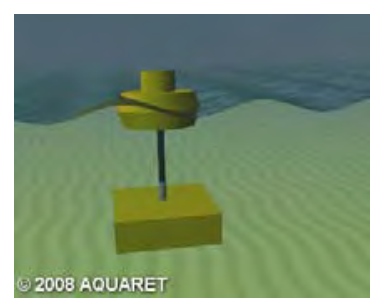

\section{b) Attenuator}

An attenuator is a floating system aligned in parallel with wave direction. Movements along its length can be selectively constrained to produce energy.

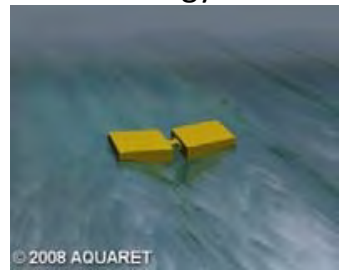

\section{c) Terminator}

A terminator is a floating system that extends perpendicularly to the wave direction, restraining waves as they arrive.

\footnotetext{
${ }^{9}$ As defined in the Review and analysis of ocean energy systems development and supporting policies, a report by AEA Energy \& Environment on the behalf of Sustainable Energy Ireland for the IEA's Implementing Agreement on Ocean Energy Systems, 28th June 2006 (http://www.iea-oceans.org/_fich/6/Review_Policies_on_OES_2.pdf) Images source: http://www.wavec.org/index.php/17/technologies/
} 


\section{d) Overtopping device}

An overtopping device contains a wall over which waves topple into a storage reservoir. The reservoir creates a head of water, which is released through hydro turbines as the water flows back out to sea. An overtopping device may use collectors to concentrate the wave energy.

\section{e) Oscillating Water Column (OWC)}

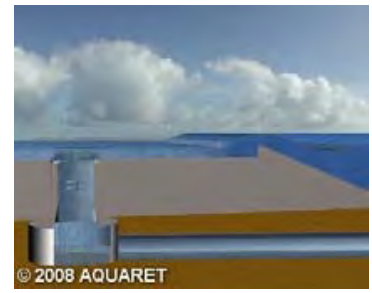

In an oscillating water column (OWC), water enters through a subsurface opening into a chamber that contains air. The wave action causes the captured water column to rise and fall like a piston, compressing and decompressing the air. This air is then channeled through an opening connected to a bi-directional turbine.

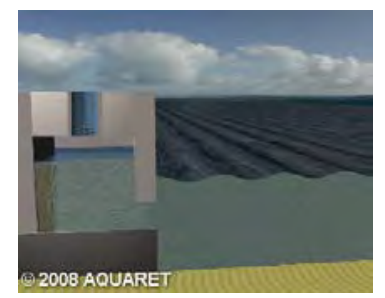

\section{f) Oscillating Wave Surge Converter (OWSC) or Pitching/Surging/Heaving/Sway (PSHS) device}

An OWSC comprises a near-surface collecting system mounted on a pivoting arm installed on the seabed. The arm oscillates like an inverted pendulum in response to the surrounding waves.

\section{g) Submerged pressure differential}

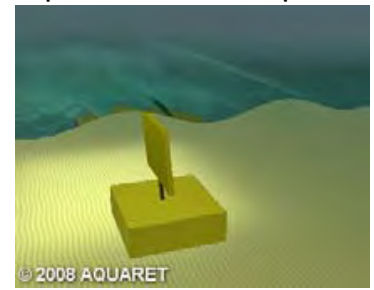

A submerged pressure differential is similar to a submerged point absorber. The passing wave induces a pressure differential within the device.

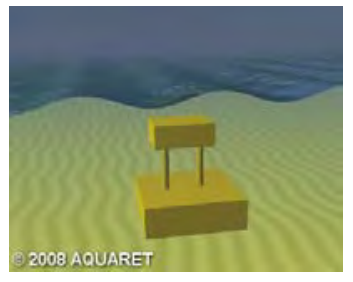

\section{h) Other}

This paragraph covers devices with a unique and very different design to the more well-established types of technology or if information on the device's characteristics could not be determined.

The following pie chart shows that the most commonly-developed technologies are point absorbers and oscillating water columns. 


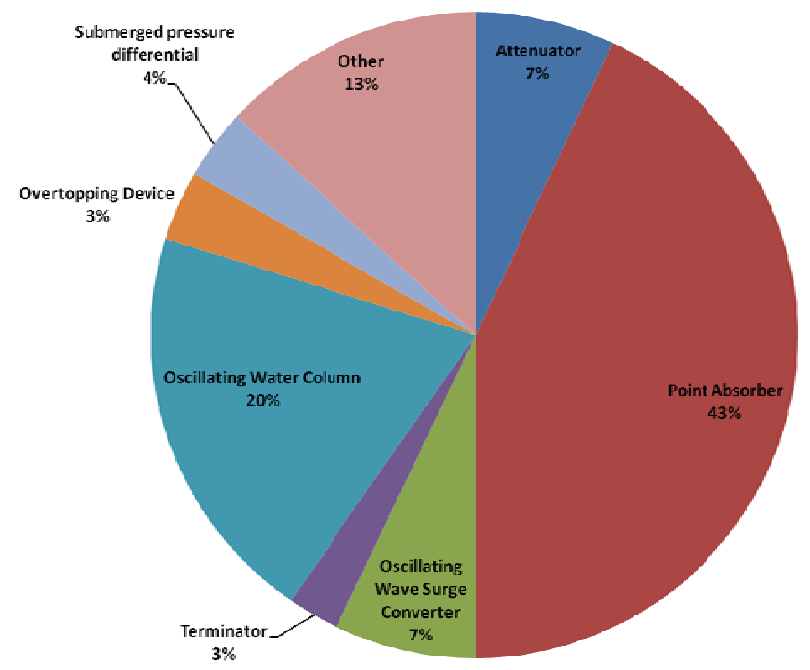

Figure 2: Technology types among wave-energy technologies

\section{Depth and Sitting Configuration}

Wave-energy devices may be installed onshore, near shore, or offshore. The distinction between near shore and onshore is not made in this analysis as the WindFloat platform is an offshore platform.

The sitting configuration, regarding the moving component of the converter, can be decomposed into four categories:

a) Floating, meaning that a component is tethered via mooring lines to the seabed, allowing freedom of movement.

b) Submerged, meaning that the device sits underwater.

c) Pile mounted, meaning that the device is attached to a pole penetrating the ocean floor.

d) Fixed, meaning that the device is attached to another fixed component or is directly fixed to the seabed.

\section{Power Take-off System}

The PTO systems in use are highly diverse. Six types of power take-off systems have been observed to convert wave energy into electricity.

\section{a) Pressurized hydraulics with water turbine or hydraulic motor.}

Pressurized hydraulics is the most common solution. The movements of wave-energy devices can directly drive a hydraulic piston in a pump or a hydraulic ram. Accumulators can also be used for energy storage. Electricity is generated by draining the pressurized accumulator via a hydraulic motor. Pressurized seawater can also be sent directly through a water turbine.

\begin{tabular}{|l|l|}
\hline \multicolumn{1}{|c|}{ ADVANTAGES } & \multicolumn{1}{c|}{ DRAWBACKS } \\
\hline Directly suitable for certain movements & Amount of hardware required (maintenance) \\
\hline Energy Storage (accumulator) & Pressure losses \\
\hline Flexibility & Environmental impacts \\
$-\quad$ water turbine if seawater is employed & $-\quad$ detrimental fluids \\
$-\quad$ single accumulator and generator for & $-\quad$ risks of leaks \\
\hline
\end{tabular}




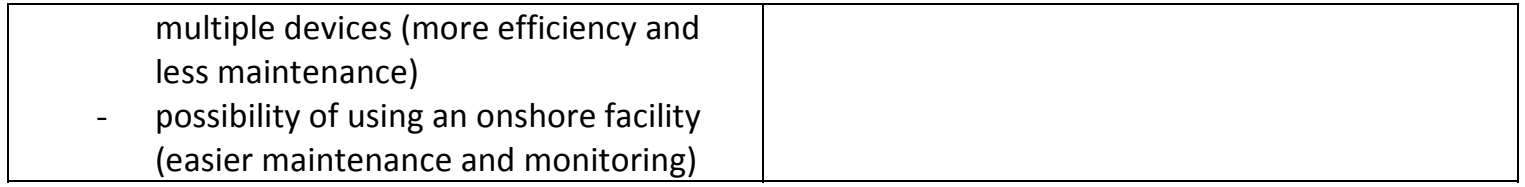

b) Mechanical

Mechanical power take-off systems come in different shapes and sizes, including worm gears or rack-and-pinion type systems for converting vertical motion into rotation, as well as clutch-flywheel or rectifying systems that convert oscillating rotation into unidirectional rotation. Conventional rotary-motion generators convert this rotational motion into electricity.

\begin{tabular}{|l|l|}
\hline \multicolumn{1}{|c|}{ ADVANTAGES } & \multicolumn{1}{c|}{ DRAWBACKS } \\
\hline High conversion efficiency & $\begin{array}{l}\text { Amount of moving parts (maintenance and } \\
\text { survivability) }\end{array}$ \\
\hline Simple rotary-motion generator & \\
\hline $\begin{array}{l}\text { Possibility to provide filtered power output (with } \\
\text { flywheels) }\end{array}$ & \\
\hline
\end{tabular}

\section{c) Linear generator}

A linear generator comprises a moving rotor containing a set of permanent magnets and a fixed stator consisting of coils arranged in a certain form.

\begin{tabular}{|l|l|}
\hline \multicolumn{1}{|c|}{ ADVANTAGES } & \multicolumn{1}{c|}{ DRAWBACKS } \\
\hline Direct conversion of movements of devices & No filtered power output \\
\hline Simple system & No storage \\
\hline $\begin{array}{l}\text { Amount of moving parts (maintenance and } \\
\text { survivability) }\end{array}$ & \\
\hline
\end{tabular}

d) Air Turbine. Usually, air turbines are used in oscillating water columns (OWC) where the air flow direction has no influence on the direction of turbine rotation.

e) Water turbine only. Usually, low-head water turbines are used in overtopping devices.

f) Others. A flexible structure, made out of materials with special characteristics (magnetostrictive or electroactive materials for instance), that changes shape or volume to generate electricity can be part of the PTO system.

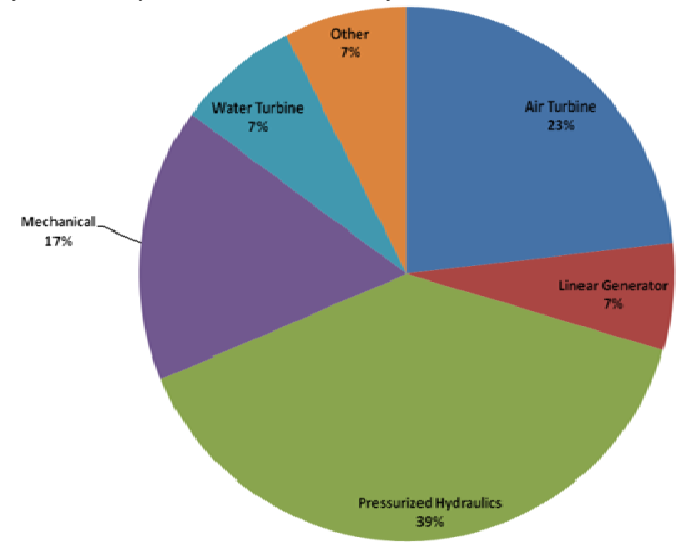

Figure 3: Power take-off system usage among wave-energy technologies 


\section{Maturity}

In general, the Technology Readiness Levels (TRLS), considered a standard measure of maturity, are used to assess the maturity level of a technology. For wave-energy technologies, all at different stages, a simplified classification including the following activities ${ }^{10}$ has been chosen in order to determine the overall status:
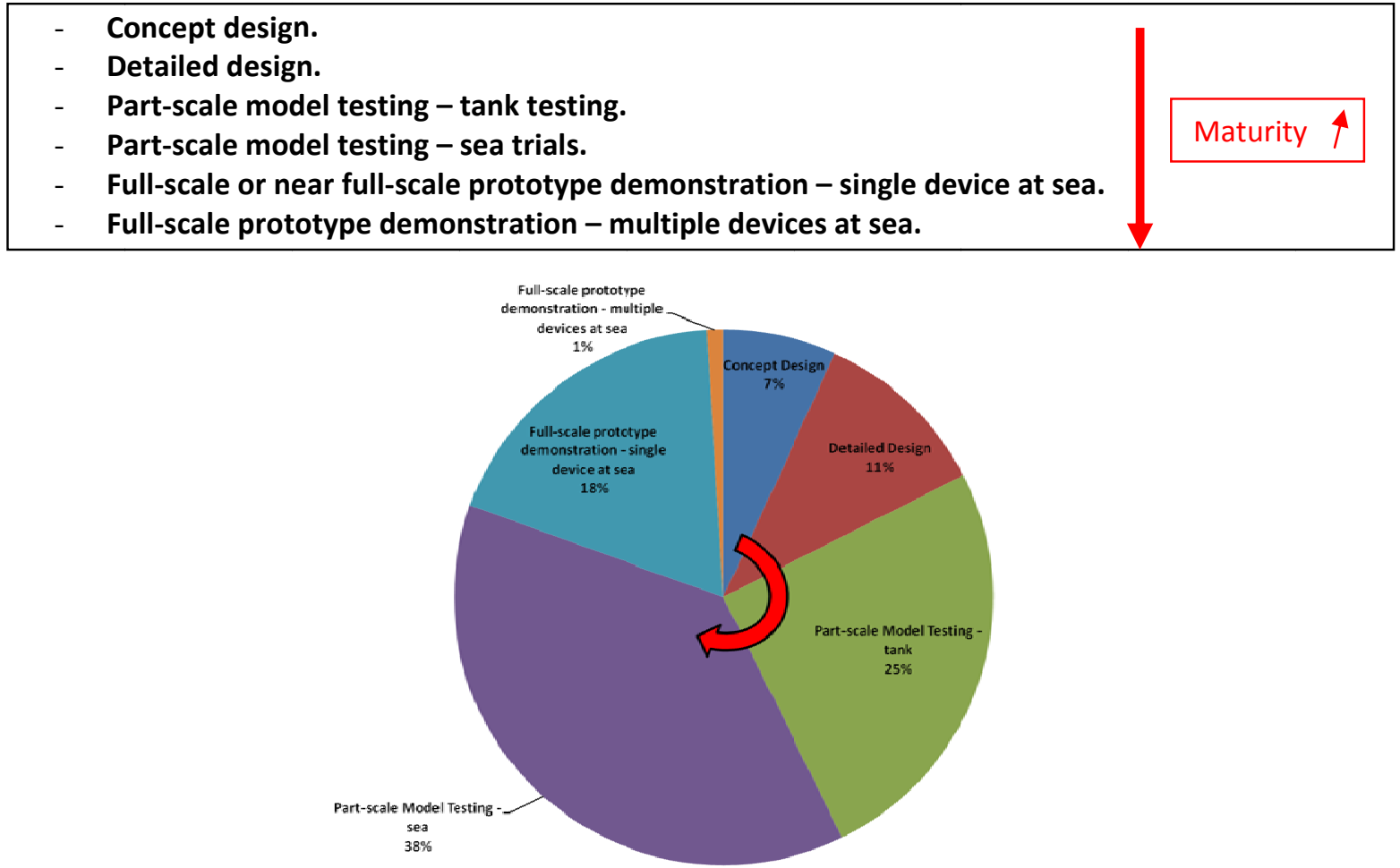

Figure 4: Percentage of technologies in each maturity category

\section{Performance}

When a wave-energy technology is assessed, its power output and efficiency is the most difficult to interpret, since it really depends on its definition and method of computation. Moreover, with the diversity of technology types and power take-off systems, it is complicated to compare the performance of one technology versus another. Standards that address diverse subjects including performances are being developed by the International Electrotechnical Commission (IEC) ${ }^{11}$. In the listing of technologies (see APPENDICES) performance metrics are reported as given by the technology developer.

\footnotetext{
${ }^{10}$ As defined in the Review and analysis of ocean energy systems development and supporting policies, a report by AEA Energy \& Environment on the behalf of Sustainable Energy Ireland for the IEA's Implementing Agreement on Ocean Energy Systems, 28th June 2006

${ }^{11}$ The IEC established the Committee IEC/TC 114 and plan to release the 62600 series standards including technical specifications, notably the technical specification 62600-100 on Performance Assessment of Wave Energy Converters.
} 


\section{CONCLUSION}

On the next page, the flowchart summarizes the current state-of-the-art regarding the development of wave-energy conversion technologies. All existing technologies are aggregated and classified by dominant categories. Each category is composed of:

- a type,

- a depth - sitting configuration,

- a PTO system,

- the highest maturity level and an illustration of the most mature wave-energy technology/ies for the category.

At present, the wave-energy industry comprises a multitude of technologies at different maturity levels. These technologies, designed for both offshore or near shore applications, have strong commonalities. Eight technology types and six power take-off systems were identified in this research. Point absorbers outnumber any other type of device, and pressurized hydraulics is used on the majority of the concepts as the PTO mechanism. Almost $2 / 3$ of the companies have already performed part-scale model testing of their technologies in the tank.

Based on the findings developed in this report, the approach for the selection of wave-energy technologies has deviated from its original course. Because of the short timeline of the project, and to take advantage of the technological knowledge and expertise in the industry, the first idea was to select and adapt an existing mature wave-energy technology. However, choosing a specific mature technology to integrate on the WindFloat is not feasible within the broad scope of this project. Instead, several generic integration designs will be conceived based on popular device types and PTO mechanisms. The assessment will include the platform constraints to make sure all generic designs are suitable for the WindFloat and meet the project objectives. 


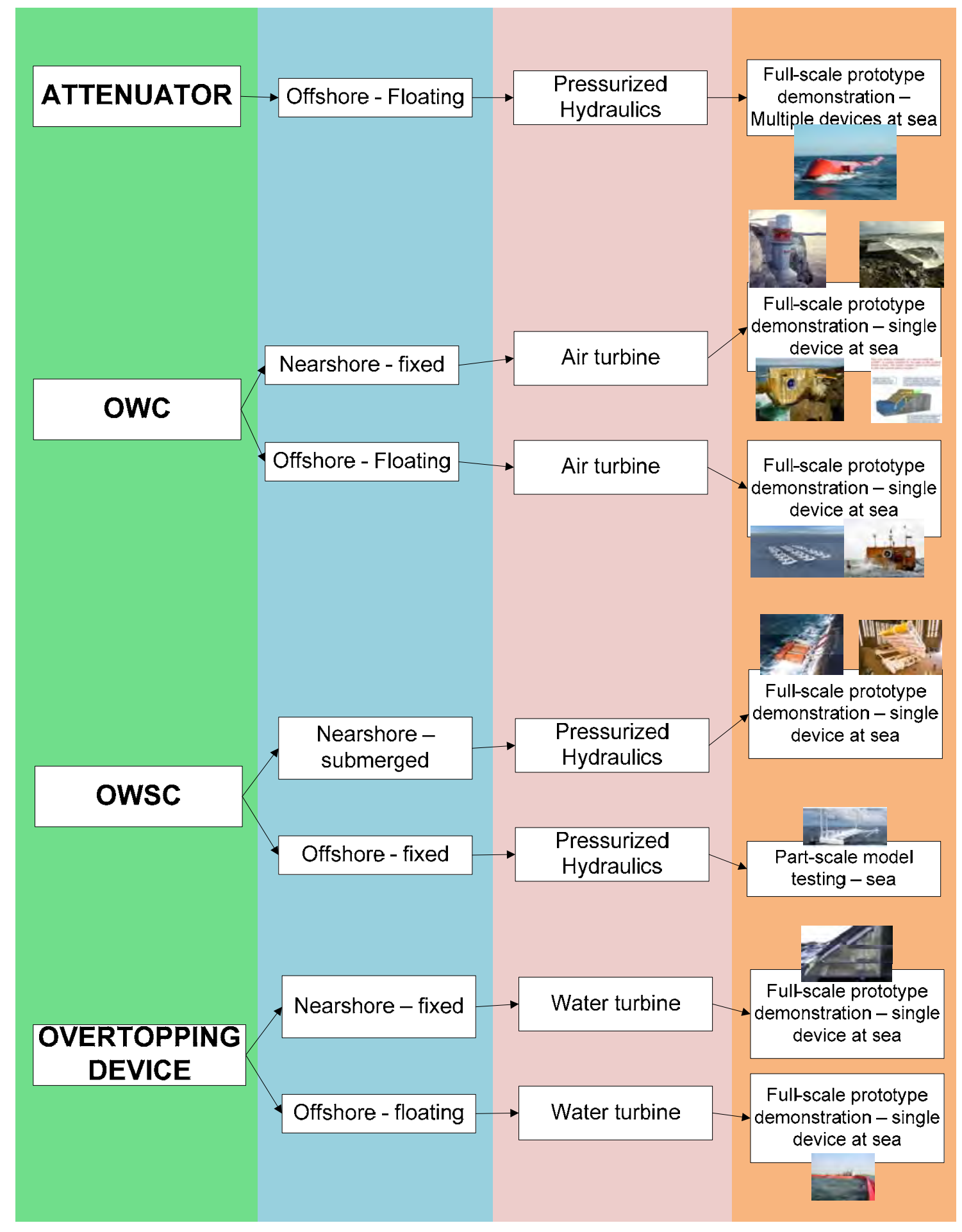

WWF - Wave-Energy PTOs State of the Art $11 / 21$ 


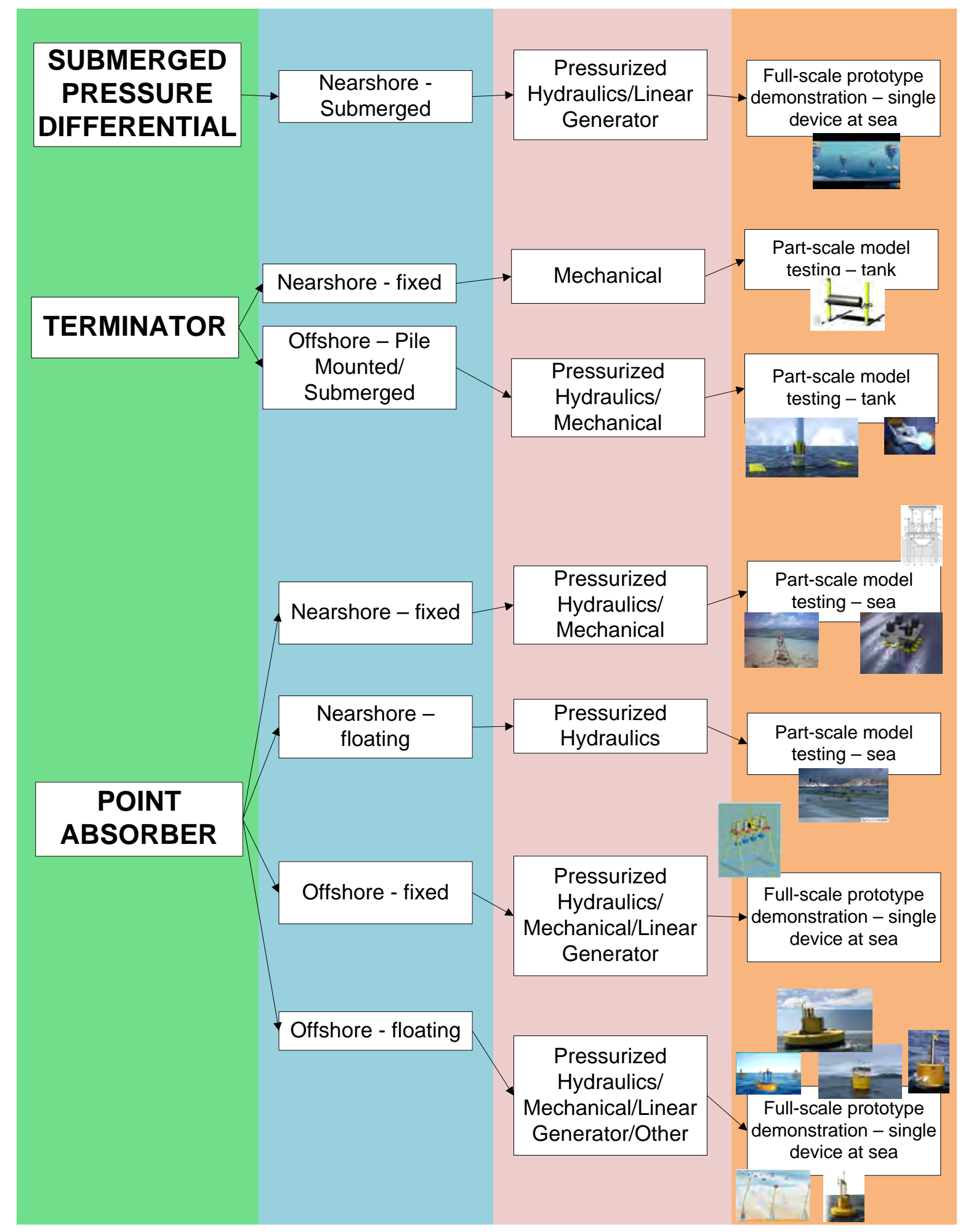

WWF - Wave-Energy PTOs State of the Art $12 / 21$ 


\section{APPENDICES}

1. Technical characteristics of an ideal wave-energy technology

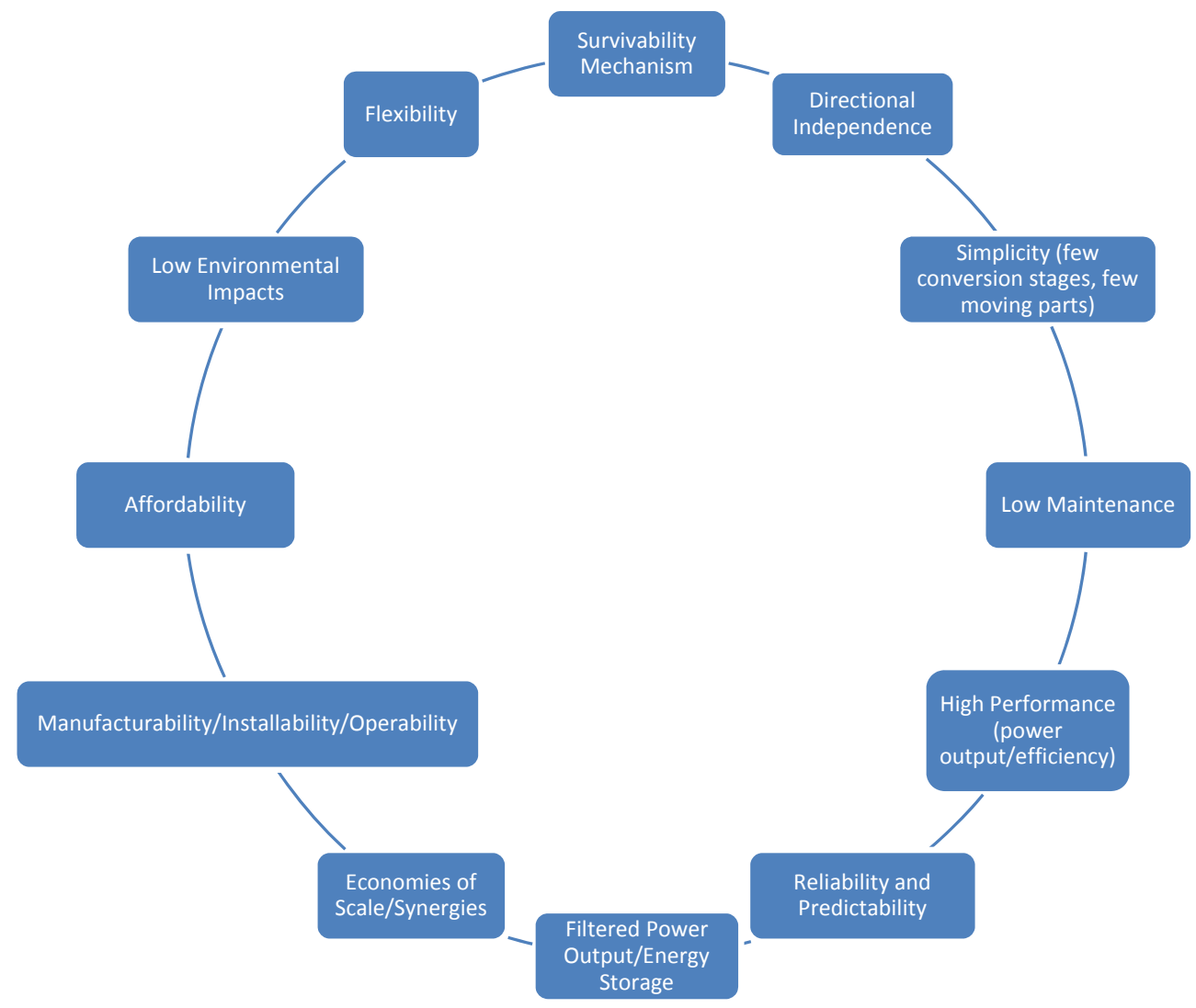

2. Wave-Energy Technology Listing

This table presents an exhaustive list of worldwide wave-energy technologies currently in development. 


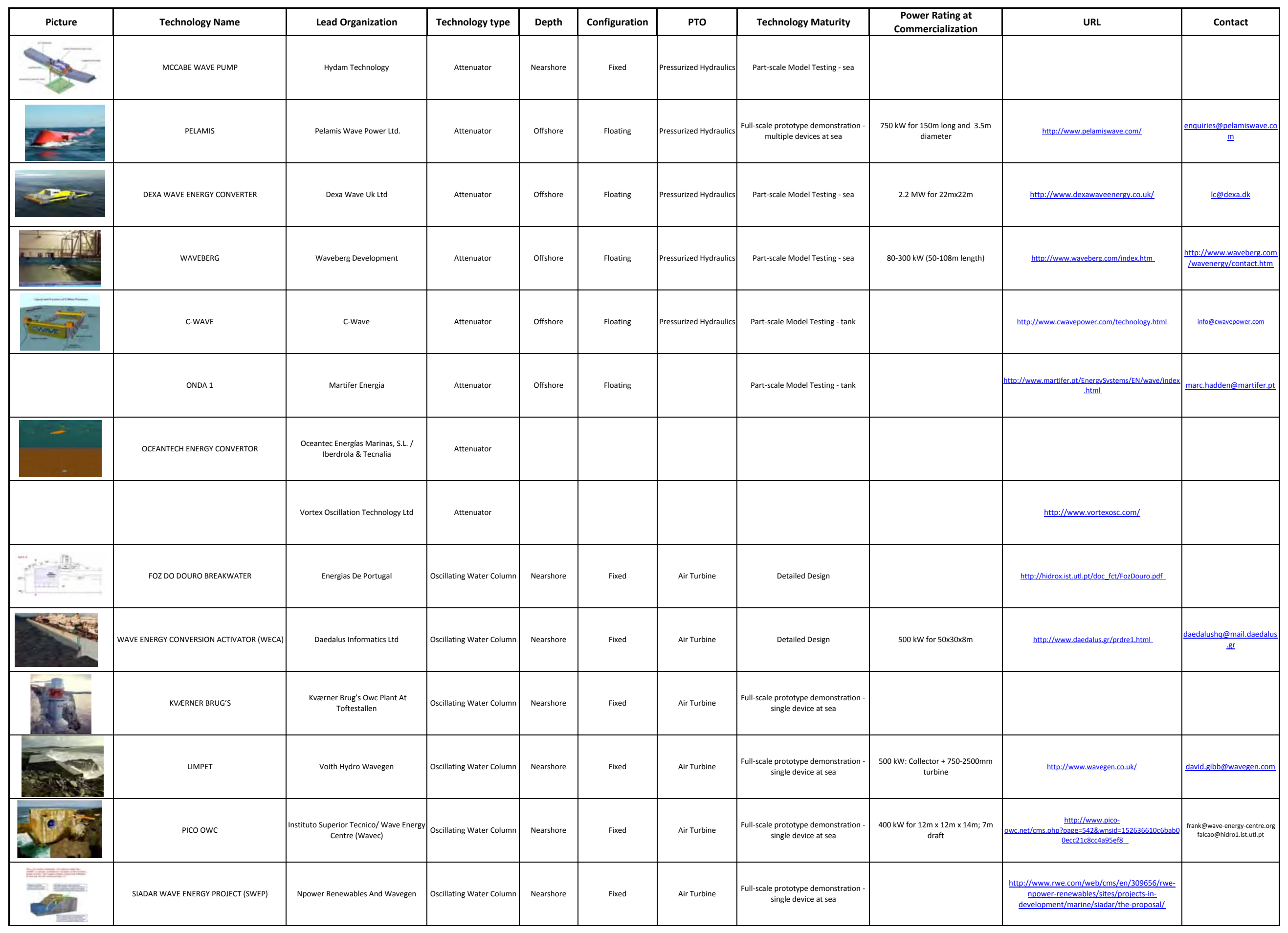




\begin{tabular}{|c|c|c|c|c|c|c|c|c|c|c|}
\hline 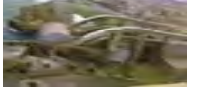 & ONSHORE OWC & $\begin{array}{l}\text { Guangzhou Institute Of Energy } \\
\text { Conversion (Giec) }\end{array}$ & Oscillating Water Column & Nearshore & Fixed & Air Turbine & Part-scale Model Testing - sea & & & \\
\hline$\Leftrightarrow$ & THE SAKATA OWC & Japanese Ministry of Transport & Oscillating Water Column & Nearshore & Fixed & Air Turbine & Part-scale Model Testing - sea & & & \\
\hline & VIZHINJAM OWC & \begin{tabular}{|l} 
National Institute of Ocean Technology \\
(Niot)
\end{tabular} & Oscillating Water Column & Nearshore & Fixed & Air Turbine & Part-scale Model Testing - sea & & & \\
\hline & Tunneled Wave Energy Converter (TWEC) & Sewave Ltd & Oscillating Water Column & Nearshore & Fixed & Air Turbine & Part-scale Model Testing - tank & & http: //www.sewave.fo/defautt.asp?sida=1380 & og@sewave.fo \\
\hline & $\begin{array}{l}\text { WIND AND OCEAN SWELL POWER (WOSP) or } \\
\text { OSPREY }\end{array}$ & Wavegen & Oscillating Water Column & Nearshore & Fixed & Air Turbine & & up to $2 \mathrm{MW}$ & 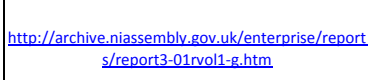 & \\
\hline & CAISSON OWC & Saga University & Oscillating Water Column & Nearshore & Fixed & Air Turbine & & & & \\
\hline & SANZE SHORELINE GULLY & Sanze Shoreline Gully & Oscillating Water Column & Nearshore & Fixed & Air Turbine & & & & \\
\hline & MK3 DENNISS-AULD TURBINE & Ocean Linx (Formerly Energetech) & Oscillating Water Column & Nearshore & Floating & Air Turbine & $\begin{array}{l}\text { Full-scale prototype demonstration - } \\
\text { single device at sea }\end{array}$ & $0.45 \mathrm{MW} ; 1.5 \mathrm{MW}$ & http://www.oceanlinx.com/ & $\begin{array}{l}\text { http://www.oceanlinx.com } \\
\text { index.php/contact-us }\end{array}$ \\
\hline & OCEAN ENERGY BUOY & Ocean Energy Ltd & Oscillating Water Column & Offshore & Floating & Air Turbine & $\begin{array}{l}\text { Full-scale prototype demonstration - } \\
\text { single device at sea }\end{array}$ & & http://www.oceanenergy.ie/ & info@oceanenergy.ie \\
\hline & BACKWARDS BENT DUCK BUOY & $\begin{array}{l}\text { Guangzhou Institute Of Energy } \\
\text { Conversion (Giec) }\end{array}$ & Oscillating Water Column & Offshore & Floating & Air Turbine & Part-scale Model Testing - sea & & & \\
\hline & MIGHTY WHALE & Jamstec & Oscillating Water Column & offshore & Floating & Air Turbine & Part-scale Model Testing - sea & & http:///www.jamstec.go.jp/jiamstec/MTD/Whale/ & washioy@jamstec.go.jp \\
\hline & MRC (MULTI RESONANT CHAMBER) & Orecon & Oscillating Water Column & Offshore & Floating & Air Turbine & Part-scale Model Testing - sea & $1.5 \mathrm{Mw}$ & http://www.orecon.com/en/the-technology// & contact@orecon.com \\
\hline & $\begin{array}{l}\text { MULTI ABSORBING WAVE ENERGY CONVERTER } \\
\text { (MAWEC) }\end{array}$ & Leancon Wave Energy & Oscillating Water Column & Offshore & Floating & Air Turbine & Part-scale Model Testing - sea & & http://www.leancon.com/technology.htm & \\
\hline & NAUTILUS & Advanced Wave Power & Oscillating Water Column & Offshore & Floating & Air Turbine & Part-scale Model Testing - sea & & http://www.advancedwavepower.com/ & \\
\hline to & PNEUMATICALLY STABILIZED PLATFORM & Float Inc. & Oscillating Water Column & Offshore & Floating & Air Turbine & Part-scale Model Testing - sea & & http://www.floatinc.com/ & \\
\hline
\end{tabular}




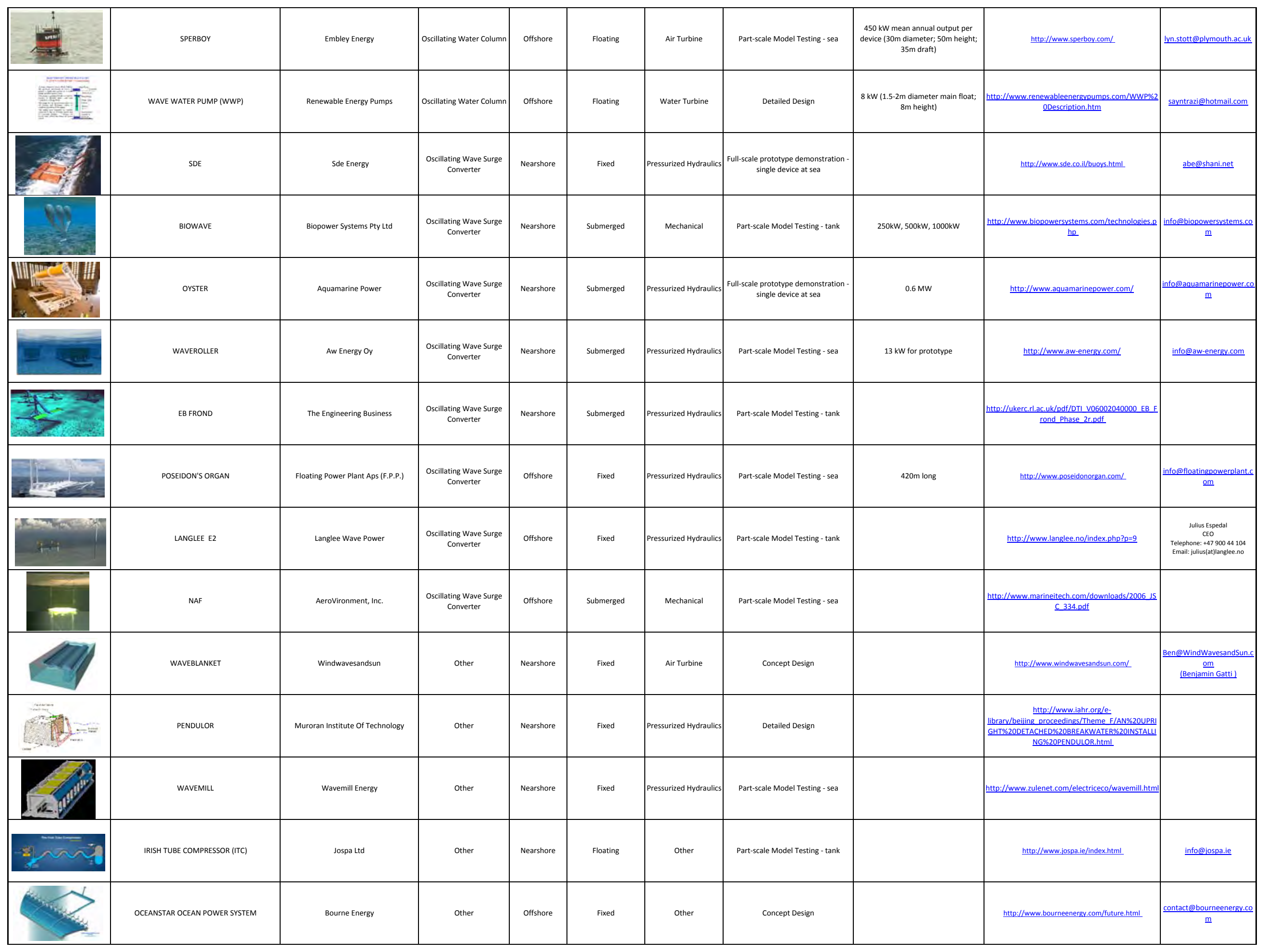




\begin{tabular}{|c|c|c|c|c|c|c|c|c|c|c|}
\hline & OWEL ENERGY CONVERTER & Offshore Wave Energy Ltd & Other & Offshore & Floating & Air Turbine & Part-scale Model Testing - sea & $10 \mathrm{MW}$ & http://owel.co.uk/print/paper2.htm & owel@svcamore.org.uk \\
\hline & WAVE CATCHER & Offshore Islands Limited & Other & Offshore & Floating & Mechanical & Concept Design & & $\frac{\text { http://www.offshoreislandslimited.com/offshore\%20isl }}{\text { ands\%20limited 006.htm }}$ & $\frac{\text { sales@offshoreislandslimit }}{\text { ed.com }}$ \\
\hline 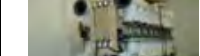 & $\begin{array}{l}\text { MHD (magnetohydrodynamics) WAVE ENERGY } \\
\text { CONVERSION (MWEC) }\end{array}$ & Sara Ltd & other & Offshore & Floating & Other & Part-scale Model Testing - tank & $100 \mathrm{~kW}$ & http://www.sara.com/RAE/ocean_wave.html & info@sara.com \\
\hline & TEtRON & Joules Energy Efficiency Services Ltd & Other & Offshore & Floating & \begin{tabular}{|l} 
Pressurized Hydraulics \\
\end{tabular} & Part-scale Model Testing - tank & & 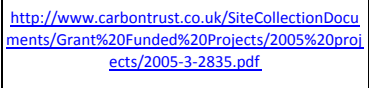 & nwells@joules.com \\
\hline & WAVEPLANE & $\begin{array}{l}\text { WavePlane Production A/S, } \\
\text { Caley Ocean Systems Ltd }\end{array}$ & Other & Offshore & Floating & Water Turbine & $\begin{array}{l}\text { Full-scale prototype demonstration - } \\
\text { single device at sea }\end{array}$ & & httpp://www.waveplane.com/ & es@waveplane.com \\
\hline & WAVE ROTOR & Ecofys & other & Offshore & Pile Mounted & Water Turbine & Part-scale Model Testing - sea & $30 \mathrm{~kW}$ for $5 \mathrm{~m}$ rotor diameter & http://www.ecofys.com/com.htm & info@ecofyss.co.uk \\
\hline & GYROWAVEGEN & Gyrowavegen & Other & Offshore & Submerged & Other & & & $\frac{\text { http://www.peswikic.com/index.php/Directory:Gyrowa }}{\text { veGen(tm) }}$ & $\frac{\text { gyrowavegen@sbcglobal.n }}{\underline{\text { et. }}}$ \\
\hline & MHD NEPTUNE & Neptune Systems & Other & Offshore & Submerged & Other & & & $\frac{\text { ftp://ftp.cordis.europa.eu/pub/sustdev/docs/energ }}{\text { y/roman_yvette.pdf }}$ & $\frac{\text { roman@neptunesystems.n }}{\underline{\text { et }}}$ \\
\hline & ANACONDA & Checkmate Seaenergy Uk Ltd. & Other & Offshore & Submerged & Pressurized Hydraulics & Part-scale Model Testing - tank & $\begin{array}{l}1 \mathrm{MW} \text { for a diameter of } 7 \mathrm{~m} \text { and } 200 \mathrm{~m} \\
\text { long }\end{array}$ & http://www.checkmateuk.com/seaenergy/system.html & $\frac{\text { info@checkmateseaenergy. }}{\underline{\text { com }}}$ \\
\hline & SEAHEART & Oceanic Power & Other & & & & & & & \\
\hline & $\begin{array}{l}\text { SSG-CONCEPT (SEA-WAVE SLOT-CONE } \\
\text { GENERATOR CONCEPT) }\end{array}$ & WAVEenergy AS, Fred Olsen & Overtopping Device & Nearshore & Fixed & Water Turbine & $\begin{array}{l}\text { Full-scale prototype demonstration - } \\
\text { single device at sea }\end{array}$ & $10 \times 22 \times 9 \mathrm{~m}$ & hittp://waveenergy.no/technology_innovation/ & $\frac{\text { espen.osaland@waveenerg }}{\underline{y . n o}}$ \\
\hline & TAPChAN & Norwave As & Overtopping Device & Nearshore & Fixed & Water Turbine & & & & \\
\hline & FLOATING WAVE POWER VESSEL & Sea Power International Ab & Overtopping Device & Nearshore & Floating & Water Turbine & Part-scale Model Testing - sea & & http://www.seapowerinternational.se/FWPV.html & contact@seapower.se \\
\hline & WAVE DRAGON & Wave Dragon & Overtopping Device & Offshore & Floating & Water Turbine & $\begin{array}{l}\text { Full-scale prototype demonstration - } \\
\text { single device at sea }\end{array}$ & $\begin{array}{l}11 \mathrm{MW} \text { for } 220 \times 390 \times 19 \mathrm{~m} ; 4,000 \mathrm{~m} 3 \\
\text { (reservoir volume) }\end{array}$ & http://www.wavedragon.net & info@wavedragon.net \\
\hline & TWPEG & $\begin{array}{l}\text { Balkee Tide And Wave Electricity } \\
\text { Generator }\end{array}$ & Point Absorber & Nearshore & Fixed & Mechanical & Part-scale Model Testing - sea & & $\frac{\text { http:///www.peswikicom/index.php/Directory:Balkee_T }}{\text { ide and Wave Electricity Generator }}$ & r.balkee@vahoo.com \\
\hline
\end{tabular}




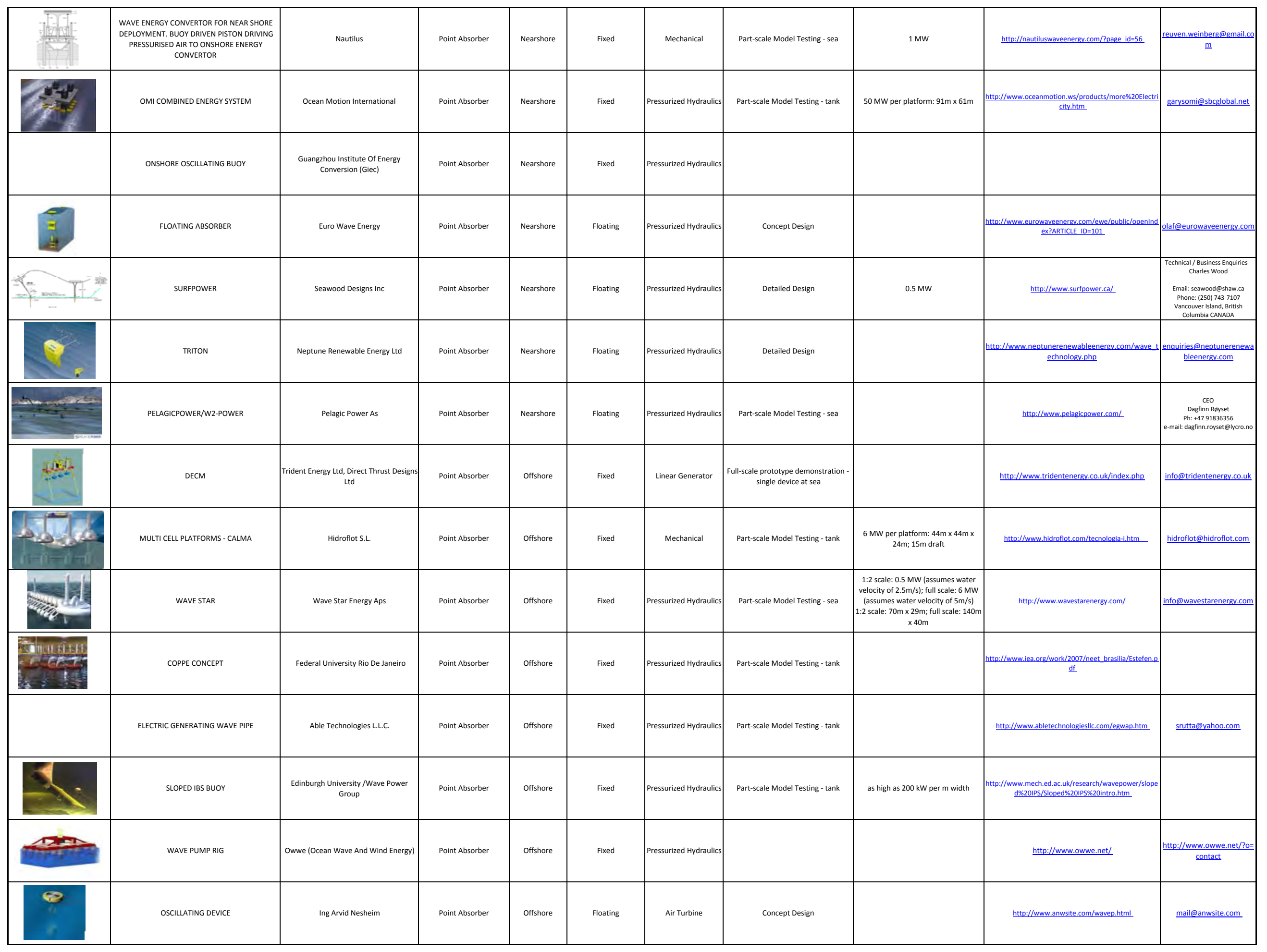




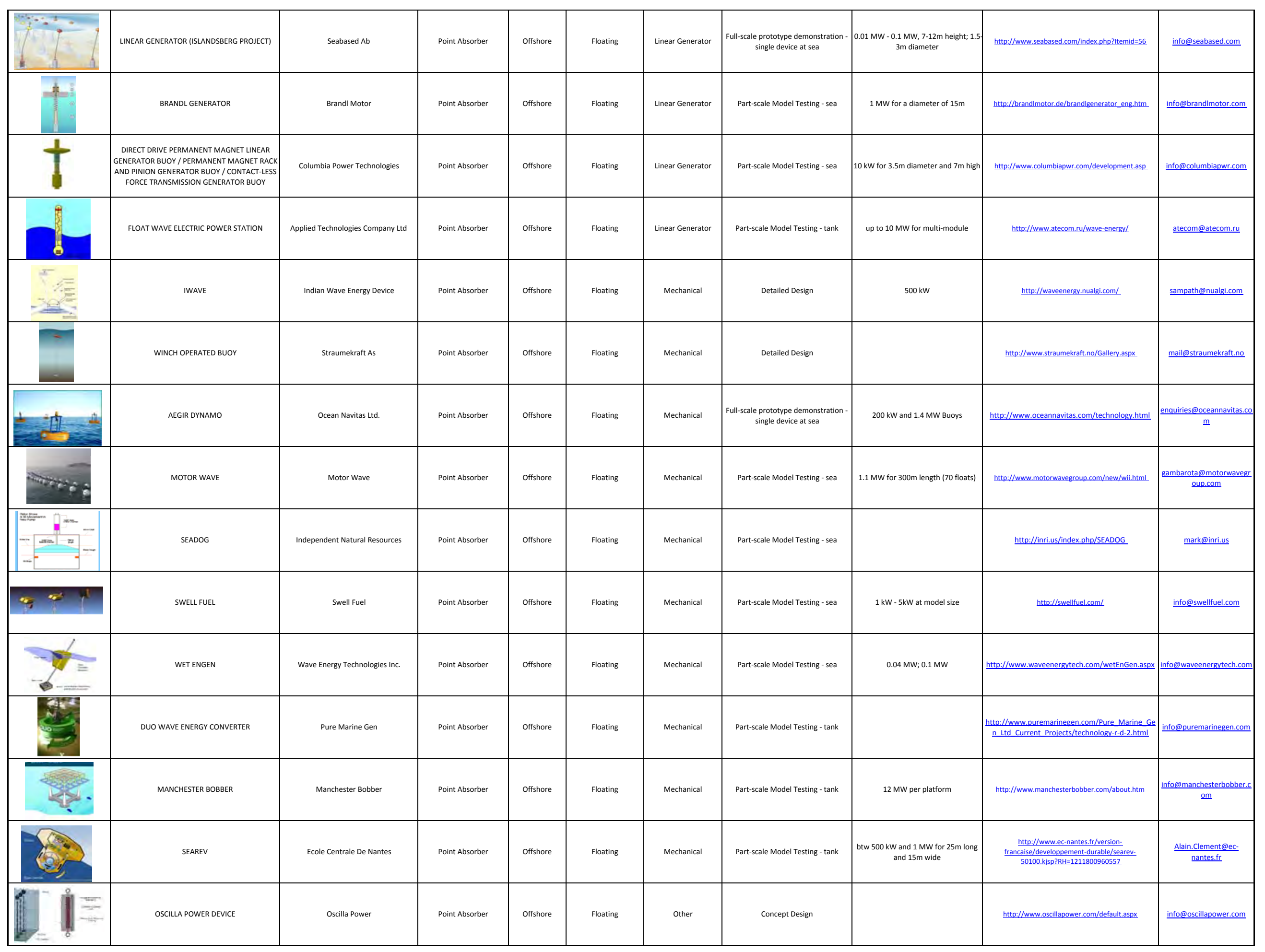




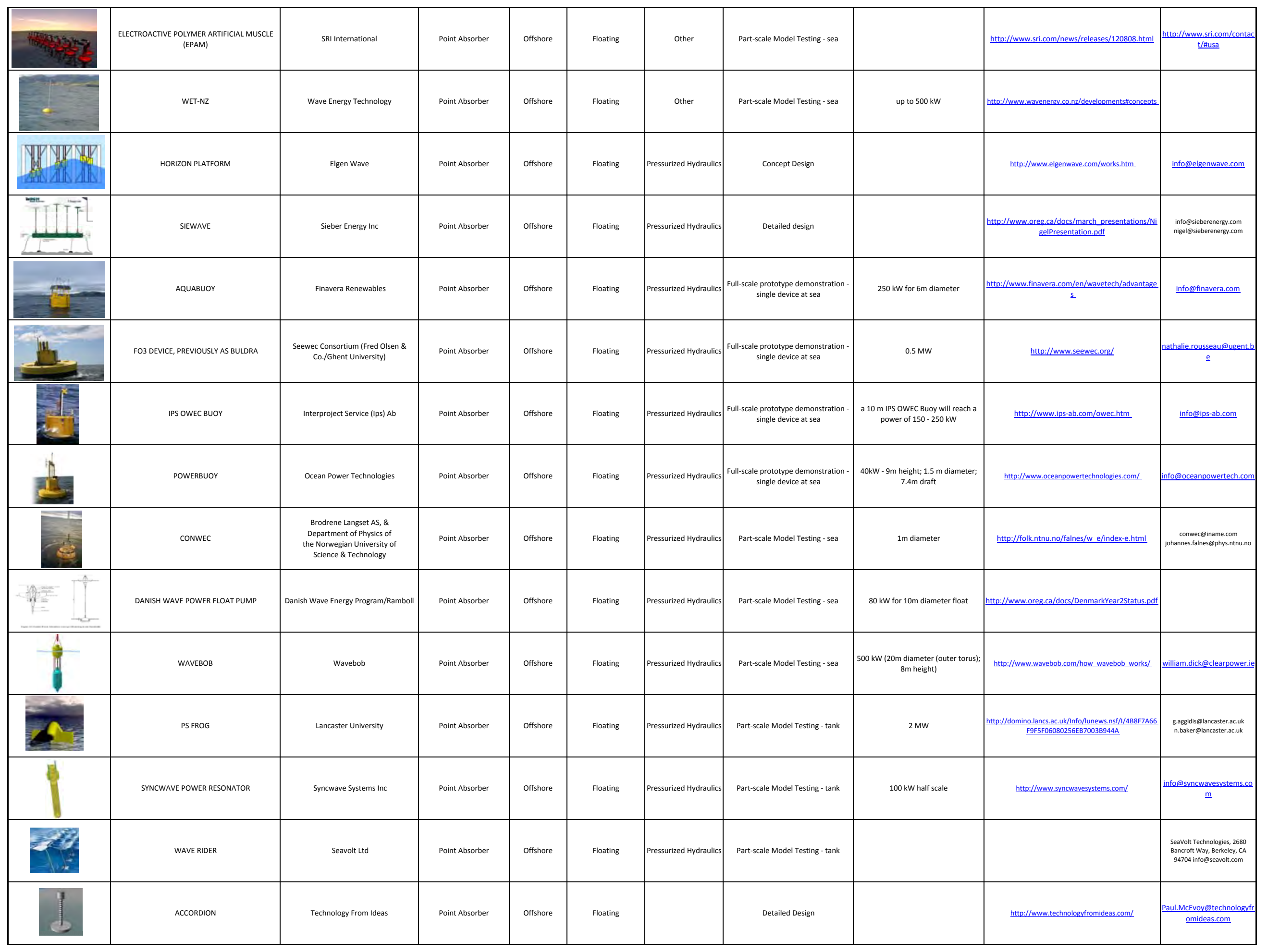




\begin{tabular}{|c|c|c|c|c|c|c|c|c|c|c|}
\hline & RESOLUTE WEC & Resolute Marine Energy, Inc. & Point Absorber & Offshore & Floating & & Part-scale Model Testing - sea & $\begin{array}{c}0.001 \mathrm{MW}-0.01 \mathrm{MW}(9 \times 3 \mathrm{~m} \text { (float } \\
\text { fully extended)) }\end{array}$ & http://web.mit.edu/alam/www/Wave Energy.htm & $\frac{\text { wstaby@resolute-marine- }}{\text { energy.com }}$ \\
\hline & SYPHON WAVE GENERATOR & Green Wave Energy Corp. & $\begin{array}{l}\text { Submerged pressure } \\
\text { differential }\end{array}$ & Nearshore & Fixed & Pressurized Hydraulics & Detailed Design & & http://www.gweconline.com/wave energy.html & \\
\hline & ARCHIMEDES WAVE SWING & Aws Ocean Energy & $\begin{array}{l}\text { Submerged pressure } \\
\text { differential }\end{array}$ & Nearshore & submerged & Linear Generator & $\begin{array}{l}\text { Full-scale prototype demonstration - } \\
\text { single device at sea }\end{array}$ & $250 \mathrm{~kW} 48 \mathrm{~m}^{*} 28 \mathrm{~m} * 38 \mathrm{~m}$ & http://www.awsocean.com/technology.aspx & info@awsocean.com \\
\hline & OWEC & Ocean Wave Energy Company & $\begin{array}{l}\text { Submerged pressure } \\
\text { differential }\end{array}$ & Nearshore & Submerged & Linear Generator & Part-scale Model Testing - sea & 5.5m high and $4 \mathrm{~m}$ wide & http://www.owec.,com/development.html & foerd@owec.com \\
\hline & WAVE POWERED PUMP & College of The North Atlantic & $\begin{array}{l}\text { Submerged pressure } \\
\text { differential }\end{array}$ & Nearshore & submerged & Pressurized Hydraulics & Part-scale Model Testing - sea & & $\frac{\text { http://www.cna.nl.ca/news/newsletters/Fall\%202006.p }}{\text { df }}$ & \\
\hline & СЕТО & Renewable Energy Holdings & $\begin{array}{l}\text { Submerged pressure } \\
\text { differential }\end{array}$ & Nearshore & Submerged & Pressurized Hydraulics & Part-scale Model Testing - sea & $190 \mathrm{~kW}$ & http://www.carnegiecorp.com.au/ & $\frac{\frac{\text { enquiries@carnegiewave.c }}{\text { om }}}{}$ \\
\hline & WAVE MASTER & Ocean Wavemaster Ltd & $\begin{array}{l}\text { Submerged pressure } \\
\text { differential }\end{array}$ & Offshore & Floating & Water Turbine & Part-scale Model Testing - tank & & & $\frac{\text { enquiries@oceanwavemast }}{\underline{\text { er.com }}}$ \\
\hline & WAVE TURBINE & Greencat Renewables & Terminator & Nearshore & Fixed & Mechanical & Part-scale Model Testing - tank & $\begin{array}{l}4 \mathrm{MW} \text { for } 50 \mathrm{~m} \text { long and } 16 \mathrm{~m} \\
\text { diameter }\end{array}$ & $\frac{\text { http://www.greencatrenewables.co.uk/waveenergy.ht }}{\mathrm{ml}}$ & $\frac{\text { info@greencatrenewables. }}{\underline{\text { co.uk }}}$ \\
\hline & OCEAN TREADER WEC & Green Ocean Energy Ltd & Terminator & Offshore & Pile Mounted & Pressurized Hydraulics & Part-scale Model Testing - tank & $500 \mathrm{~kW}$ for $50 \mathrm{~m}$ long and $20 \mathrm{~m}$ beam & $\frac{\text { http://www.greenoceanenergy.com/index.php/wave. }}{\text { treader }}$ & $\frac{\text { info@greenoceanenergy.cc }}{\underline{m}}$ \\
\hline & FLOATING WAVE GENERATOR & Glen Edward Cook & Terminator & offshore & Submerged & Mechanical & Part-scale Model Testing - tank & $250 \mathrm{~kW}$ for $20^{\prime} \times 46^{\prime} \times 8^{\prime}$ & http:///www.gedwardcook.com/ & G@GEdwardCook.com \\
\hline
\end{tabular}


Appendix B: Numerical Modeling of Wave Devices 


\section{(73) $P$ renewable energy delvered}

DOE Grant to Support the Development of Advanced Water Power Technologies

\section{WindWaveFloat (WWF)}

WWF CONCEPT DESIGN DELIVERABLE \#2

Numerical Modeling of Wave Devices

\section{DOE GRANT DE-EE0002652 \\ $2^{\text {nd }}$ Quarter Reporting}

July 2010 
Table of Contents

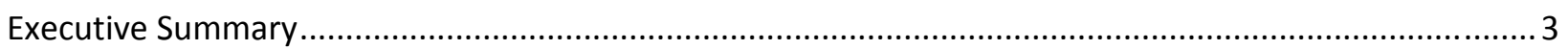

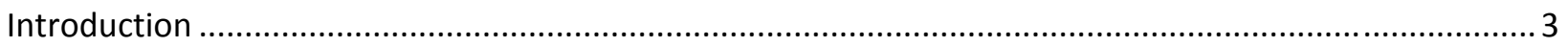

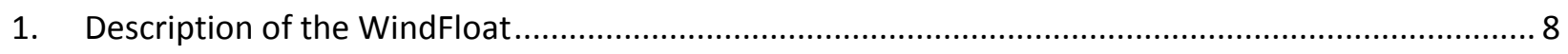

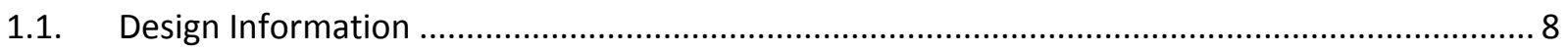

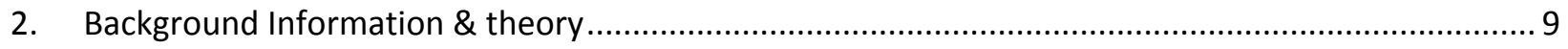

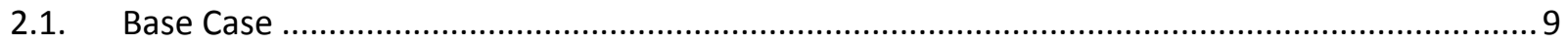

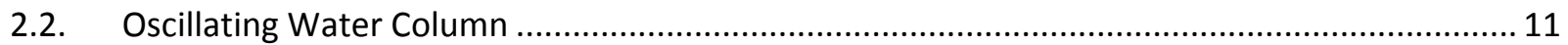

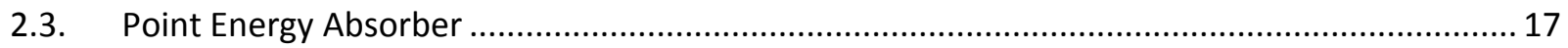

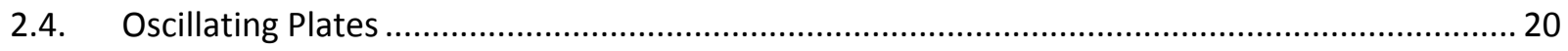

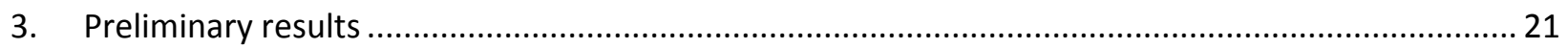

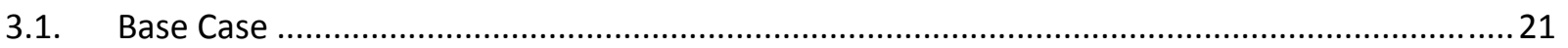

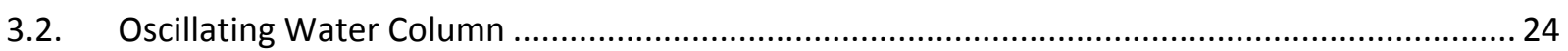

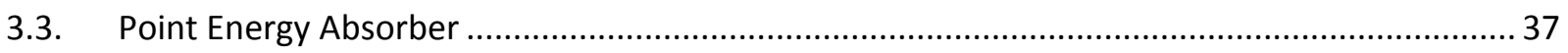

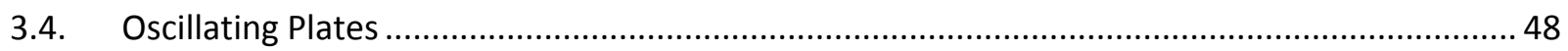

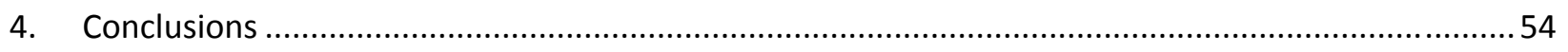

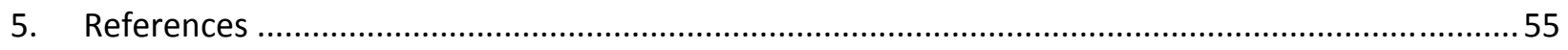

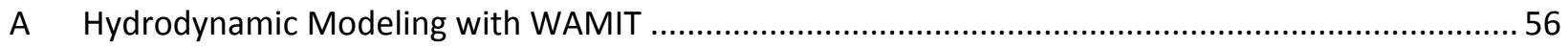

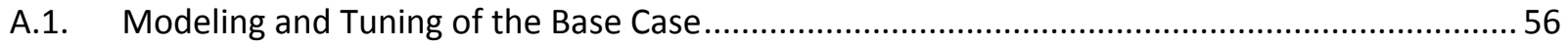

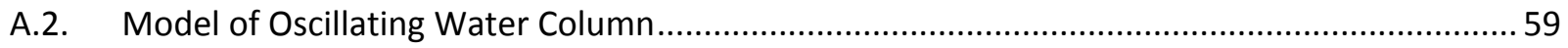




\section{Executive Summary}

This report summarizes the theory behind the modeling that was performed to incorporate Wave energy Converters (WEC) into the WindFloat. Models were developed using the diffraction-radiation code WAMIT and the time domain code ORCAFLEX. For each device analyzed, the modeling is first described, then the motion Response Amplitude Operators (RAOs) are computed. From these motion responses, the theoretical power available is calculated. The power values depend on some empirical coefficients that need to be confirmed through model testing in the lab. The forces on each device are often dependent on the interference between the device and the hull, the mooring, and the non-linear effects which are challenging to model. Therefore, these forces are approximated using a Morrison-type formulation. The empirical values for drag coefficients, damping and stiffnesses in this report are based on our "best engineering judgment" and can be seen as a "first pass", used to validate that the numerical models are working.

The next phase of this work is to perform experimental model tests, to validate the numerical models presented herein. Once that task is performed and completed, it will be possible to assess the amount of power that each device can produce. In the meanwhile, it is strongly suggested not to draw any hasty conclusions based on the power values presented in the result section of this report.

\section{Introduction}

Under DOE award DE-EE0002652, Principle Power is studying the effect of adding Wave energy device(s) to the WindFloat hull. The study is framed around understanding the economic impact of adding an additional source of energy production, at a lower cost than in their stand-alone forms, since mooring, electrical infrastructure and other components are being shared with the existing WindFloat.

It was originally thought that incorporating existing technologies by partnering with specific companies and developing their concepts would be a good avenue for this upcoming phase. However, during the development of the Wave energy PTO state-of-the-art report [1], it became clear that commercial interests, rather than technological advancements, were dominating initial discussions. There are multiple companies working on similar concepts, and each of these companies, with a vested interest in promoting their own technologies were more focused on IP protection than on the incorporation of the PTOs. It was therefore decided to study generic types of wave energy PTOs and focus on the technical integration at this stage of the work. Five types of PTO's are studied with the scope of this work. Each is integrated independently into a base case WindFloat, designed to support the generic NREL $5 \mathrm{MW}$ wind turbine [3] for worldwide environments. Figure 1 shows an artistic rendering of the WindFloat $5 \mathrm{MW}$ prototype base case. The modeling efforts of the integration of the first four PTOs are reported here. The independent effort of the integration of the $5^{\text {th }}$ device is the M.S. topic of a UC Berkeley graduate student, which is partly funded under this DOE grant. His efforts are not part of this report. Each wave technology represents a different mechanism and is designed to reflect current developments in the industry. The different technologies investigated here are: 
1. Oscillating Water Column. In an oscillating water column (OWC), water enters through a subsurface opening into a chamber that contains air. The wave action causes the captured water column to rise and fall like a piston, compressing and decompressing the air. This air is then channeled through an opening connected to a bi-directional turbine. In this case, the chamber is built externally around the two columns not supporting the turbine, as shown in Figure 2.

2. Single Point Energy absorber. A point absorber is a floating system that absorbs energy in all direction through its movements at the water surface. A point absorber is usually designed to resonate so that its harnessed power is maximized. In this generic study, we look at a sphere placed in the middle of the WindFloat triangle. The sphere was chosen because it responds well in heave, with very little pitch motion. It is shown in Figure 3.

3. Multiple point energy absorbers. A significant question with point absorber technology integration is the influence of device size vs. quantity. Is it better to have multiple smaller units, or one bigger one? Therefore 3 devices whose total displacement is the same as option 2 are being investigated, and the structure is shown in Figure 4. The results can be used to optimize the number of devices.

4. Flaps or Oscillating Wave Surge Converter (OWSC). The OWSC comprises a near-surface collecting system mounted on a pivoting arm installed on the seabed. The arm oscillates like an inverted pendulum in response to the surrounding waves. In this case the plates are mounted on the structure main beams, outside of the water. This has the significant advantage of having the possibility of being completely removed from any wave actions during large storms, as the beams are designed to be dry and the flaps should be able to be locked in an horizontal position. A rendering is shown in Figure 5. 


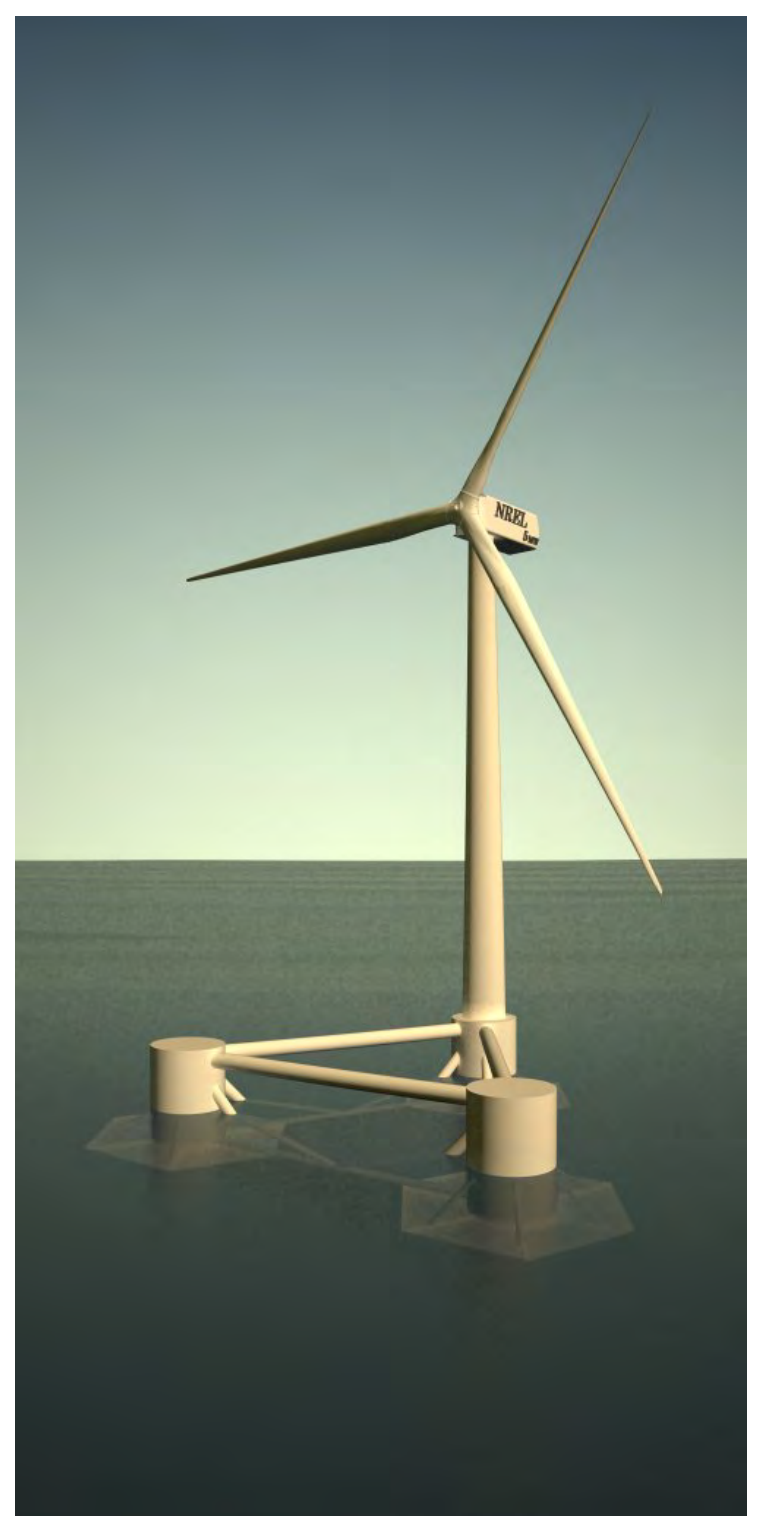

Figure 1: General View of the Generic WindFloat used as a Base Case 


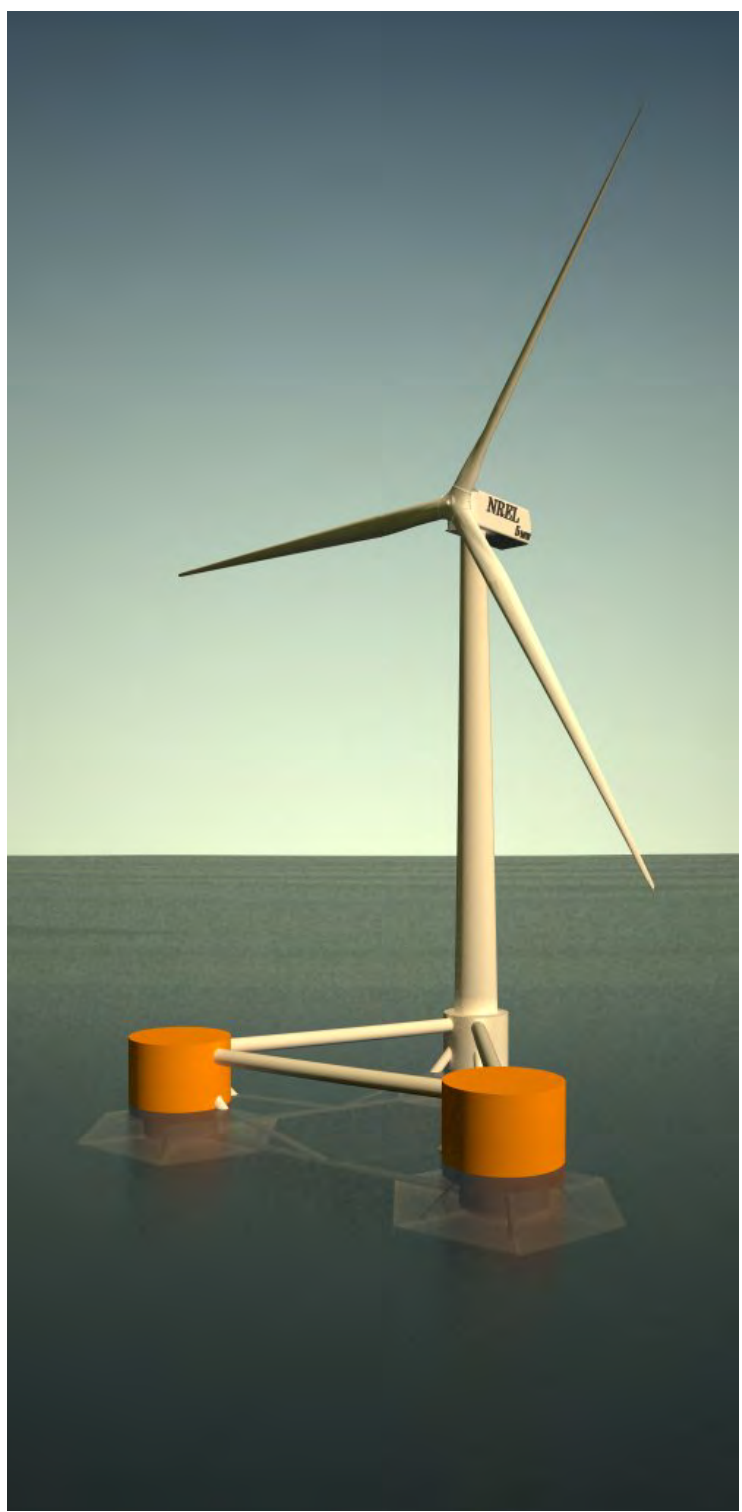

Figure 2: WindWaveFloat configuration 1: Oscillating Water column

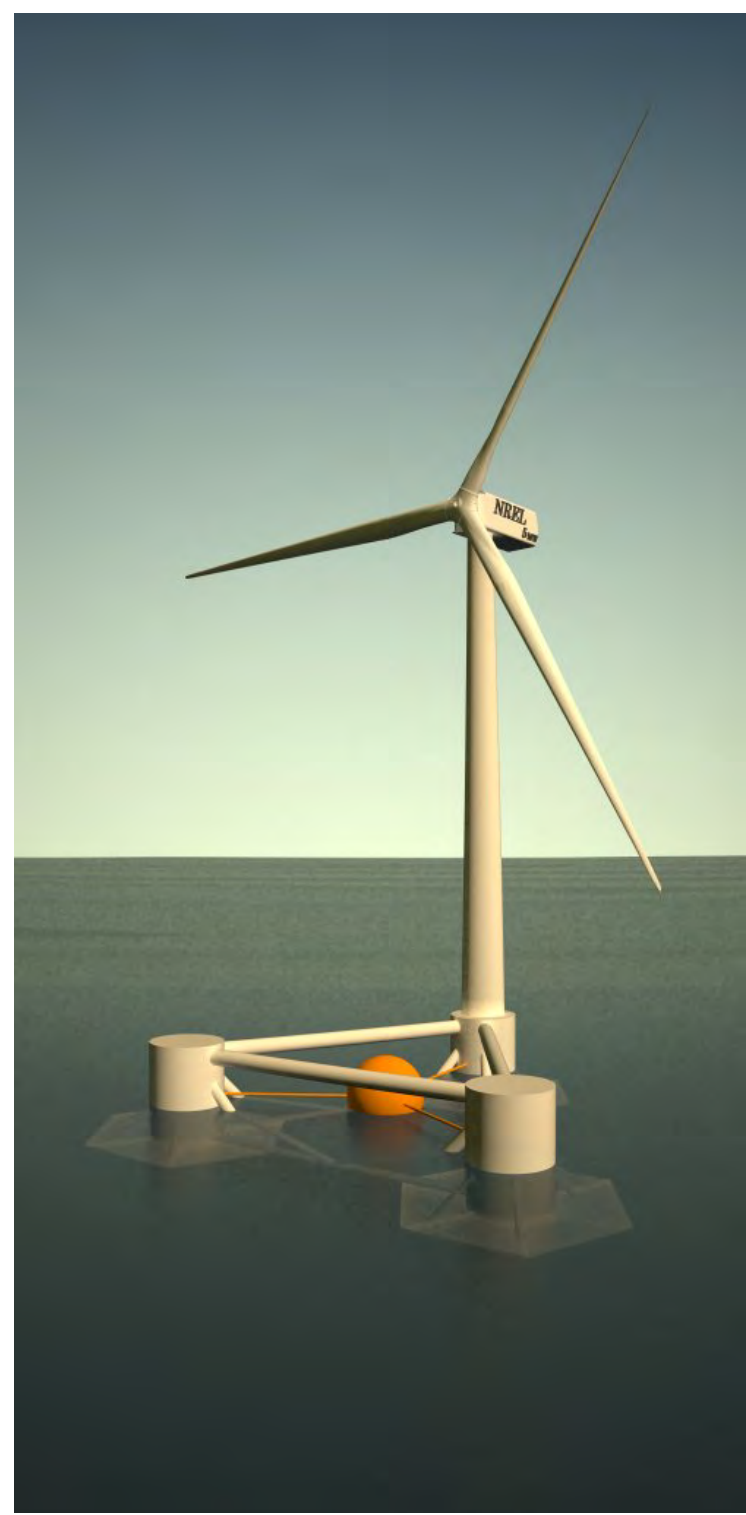

Figure 3: WindWaveFloat configuration 2, single point absorber (SWEDE) 


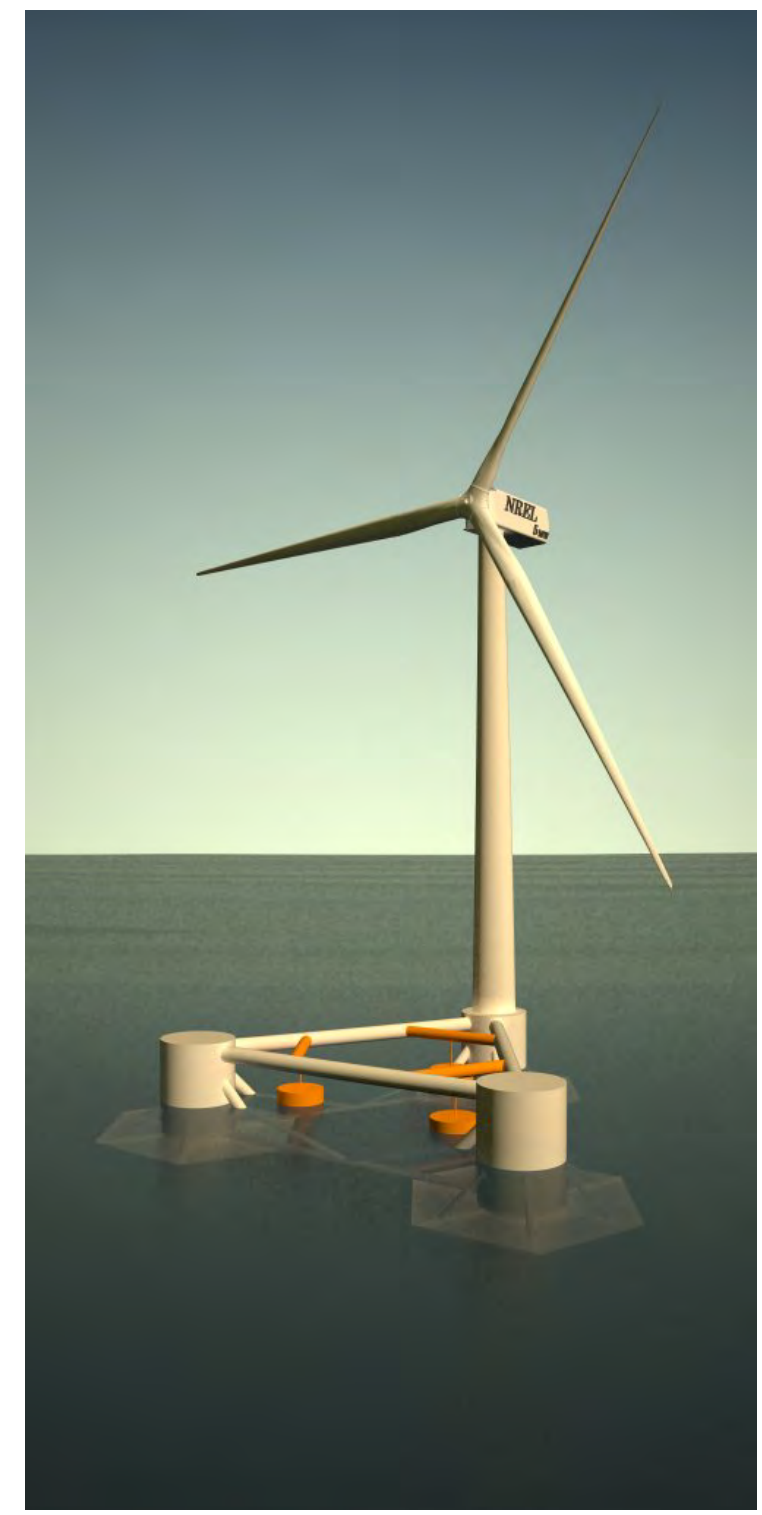

Figure 4: WindWaveFloat configuration 3, multiple point absorber.

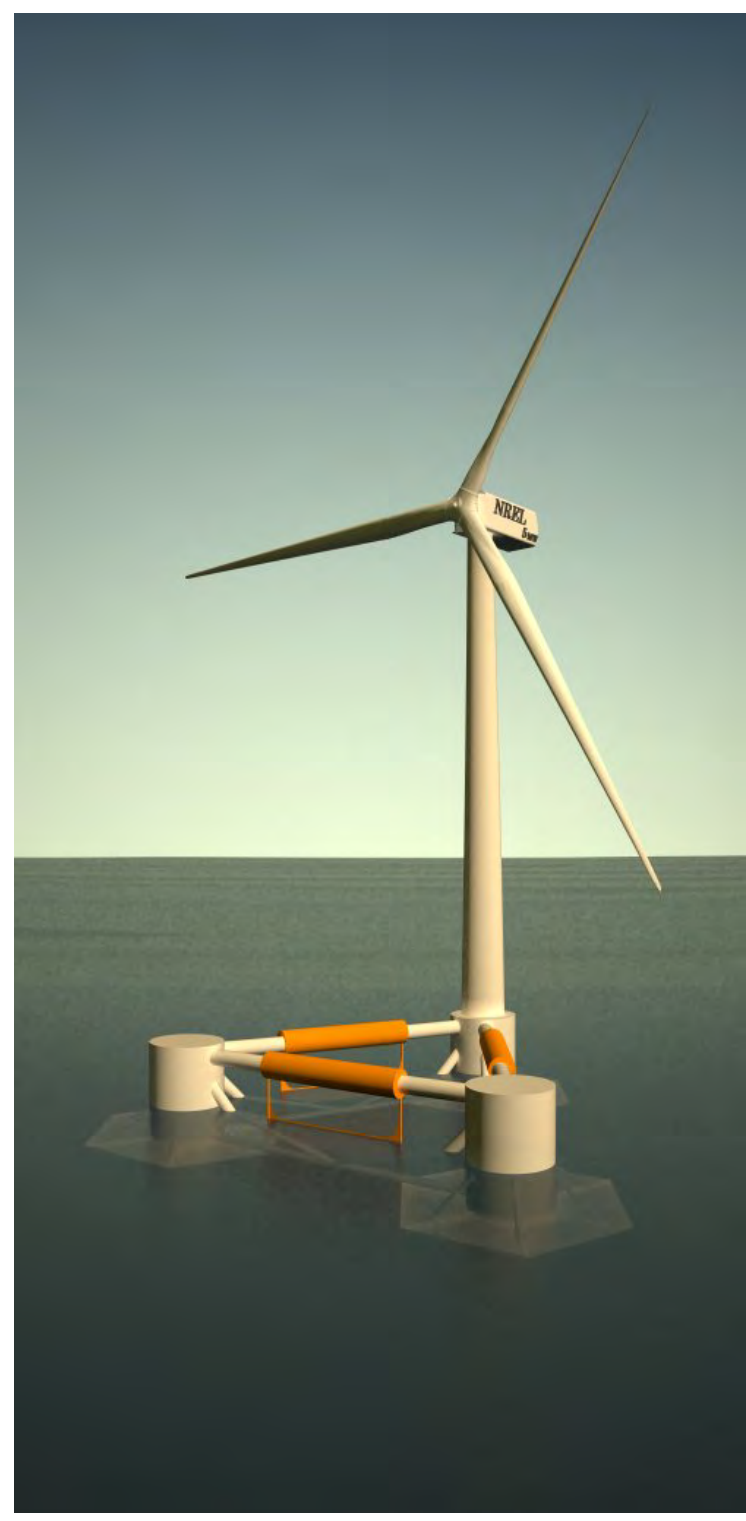

Figure 5: WindWaveFloat configuration 4, vertical oscillating plates.

This report presents the theory behind the numerical modeling, describes the models and shows some preliminary findings and results. It must be noted that all these models have some empirical components that need to be validated by experimental tests, which are planned for the next quarter. It is therefore important not to take the power prediction at face value, but focus more on the global behavior of each device. 


\section{Description of the WindFloat}

\subsection{Design Information}

The WindFloat 5MW base case hull used in this report is a generic WindFloat, developed by Principle Power Inc. (PPI), based on the experience of PPI's various projects. This generic $5 \mathrm{MW}$ WindFloat supports the publicly available NREL $5 \mathrm{MW}$ turbine (a very realistic but never built turbine) described in [3]. This base case version of the WindFloat used in the study is also being used by NREL in the OC-IV modeling work, with the same 5 MW NREL wind turbine.

NREL is leading a multinational effort on the validation of tools for the design and engineering of offshore wind. This program, [2] Offshore Code Comparison Collaboration within IEA Wind Task 23, referred to as OC-III, looked at a spar floating structure. The next phase, OC-IV will investigate a semisubmersible (WindFloat), which is more complicated to model due to the hydrodynamic radiation diffraction effects.

Extensive development efforts have been done on the WindFloat. A summary of the engineering and design of the first $5 \mathrm{MW}$ version of the WindFloat is described in [4]. Similar work was performed for this version.

\subsubsection{WindWaveFloat characteristics}

The following table (Table 1) presents the platform main dimensions. This generic WindFloat is designed to support the NREL $5 \mathrm{MW}$ wind turbine, whose properties can be found in [3] .

Table 1: WindWaveFloat Prototype dimensions

\begin{tabular}{|lll|} 
WindFloat Main Dimensions & & \\
column diameter & 10 & $\mathrm{~m}$ \\
length of water entrapment plate edge & 15 & $\mathrm{~m}$ \\
column center to center & 46 & $\mathrm{~m}$ \\
pontoon diameter & 2.1 & $\mathrm{~m}$ \\
operating draft & 17 & $\mathrm{~m}$ \\
airgap & 10 & $\mathrm{~m}$ \\
bracing diameter & 1.5 & $\mathrm{~m}$ \\
DISPLACEMENT & 4832 & tonnes \\
\hline
\end{tabular}

The WindWaveFloat is positioned with a catenary mooring, which consists of 4 mooring lines, two on column 1, which carries the turbine, and one on each other column. Each line is made of 3 sections: 3 inch chain at the fairlead, 5-inch polyester, and 3-inch chain to the anchor at the bottom. A clump weight is placed between the upper chain section and the polyester rope to control the tension. The pretension on the mooring lines is $535 \mathrm{kN}$. The displacement of the platform is 4832 metric tonnes. Mass properties are summarized below. 
Table 2: Mass Properties of the WindFloat

\begin{tabular}{|l|c|c|l|}
\hline Mass & $\mathbf{m}$ & $4.64 \mathrm{E}+06$ & $\mathrm{Kg}$ \\
\hline \multirow{3}{*}{$\begin{array}{l}\text { Coordinates } \\
\text { of the Center } \\
\text { of Gravity }\end{array}$} & $\mathbf{X g}$ & -0.278 & $\mathrm{M}$ \\
\cline { 2 - 4 } & $\mathbf{Y g}$ & 0.000 & $\mathrm{M}$ \\
\cline { 2 - 4 } Gyradii & $\mathbf{Z g}$ & 3.728 & $\mathrm{M}$ \\
\cline { 2 - 4 } & $\mathbf{R x}$ & 34.900 & $\mathrm{M}$ \\
\cline { 2 - 4 } & $\mathbf{R y}$ & 34.700 & $\mathrm{M}$ \\
\hline
\end{tabular}

\subsubsection{Site location}

The Base Case WindFloat is designed for conditions similar to the OC-III and OC-IV design basis. In the OC-III work, the generic water-depth was $325 \mathrm{~m}$. For simplicity purposes, the WindWaveFloat mooring system is designed for this same water depth. The NREL OC-III and IV do not have a specific geographical location, and their design basis assumes looking at monochromatic waves of different wave period and height. The largest waves roughly coincide with Oregon type conditions as shown in Table 3, which is the WindWaveFloat chosen location for this study.

Table 3: 100-year storm design values

\begin{tabular}{|l|l|}
\hline Sea state & 100 year storm \\
\hline Significant wave height & $44.25 \mathrm{ft}(13.5 \mathrm{~m})$ \\
\hline Peak period & $17 \mathrm{~s}$ \\
\hline Wind speed at $10 \mathrm{~m}$ elevation & $85 \mathrm{ft} / \mathrm{s}(25.9 \mathrm{~m} / \mathrm{s})$ \\
\hline Current speed & $2.6 \mathrm{ft} / \mathrm{s}(0.8 \mathrm{~m} / \mathrm{s})$ \\
\hline
\end{tabular}

\section{Background Information \& theory}

\subsection{Base Case}

\subsubsection{Two equivalent numerical models}

Two numerical models of the base case are developed for use with the different types of wave energy device.

- In WAMIT, a frequency domain diffraction-radiation program, the behavior of the platform in incident waves is determined based on linear theory.

A high order representation of the geometry is used. The water entrapment plates are thin plates represented with dipoles. The submerged columns and main beams are modeled up to the waterline.

The mass matrix is based on the platform mass properties as defined in Section 1.1.1. An equivalent damping and stiffness matrix are used to model respectively the effect of viscous damping on the water entrapment plates and the effect of mooring. These matrices are adjusted based on the Orcaflex model 
described below, and have been validated by multiple model tests. The matching between the two models is described in Appendix A.1.

- A model of the WindFloat is also generated in Orcaflex, a time-domain solver for the 6-degree of freedom equations. The response of the system to wave dynamics is obtained from WAMIT. Dynamic coefficients, such as added mass, damping and hydrostatic stiffness are transferred from the WAMIT output files, as well as diffraction forces and quadratic drift coefficients. Non-linear forces are added to the 6 degree of freedom equations as needed. Mooring components are modeled in 3D. The effect of viscosity on the columns and water entrapment plates is represented with Morison's formulas. A $C_{d}$ of 1 is used on the columns and a $C_{d}$ of 7.5 is assigned to the water entrapment plates. These values are based on previous WindFloat model tests.

\subsubsection{Coordinate System}

The system of coordinates for both models is based on the WAMIT nomenclature.

Its origin is at the waterline, at the center of the tripod formed by the three columns as illustrated in Figure 6. The axis $z$ is vertical oriented upward.

The wave heading is the direction toward which the wave is moving. It is expressed in degrees, counter clockwise from the $x$-axis direction. 0 degree wave headings only are considered in this preliminary analysis.
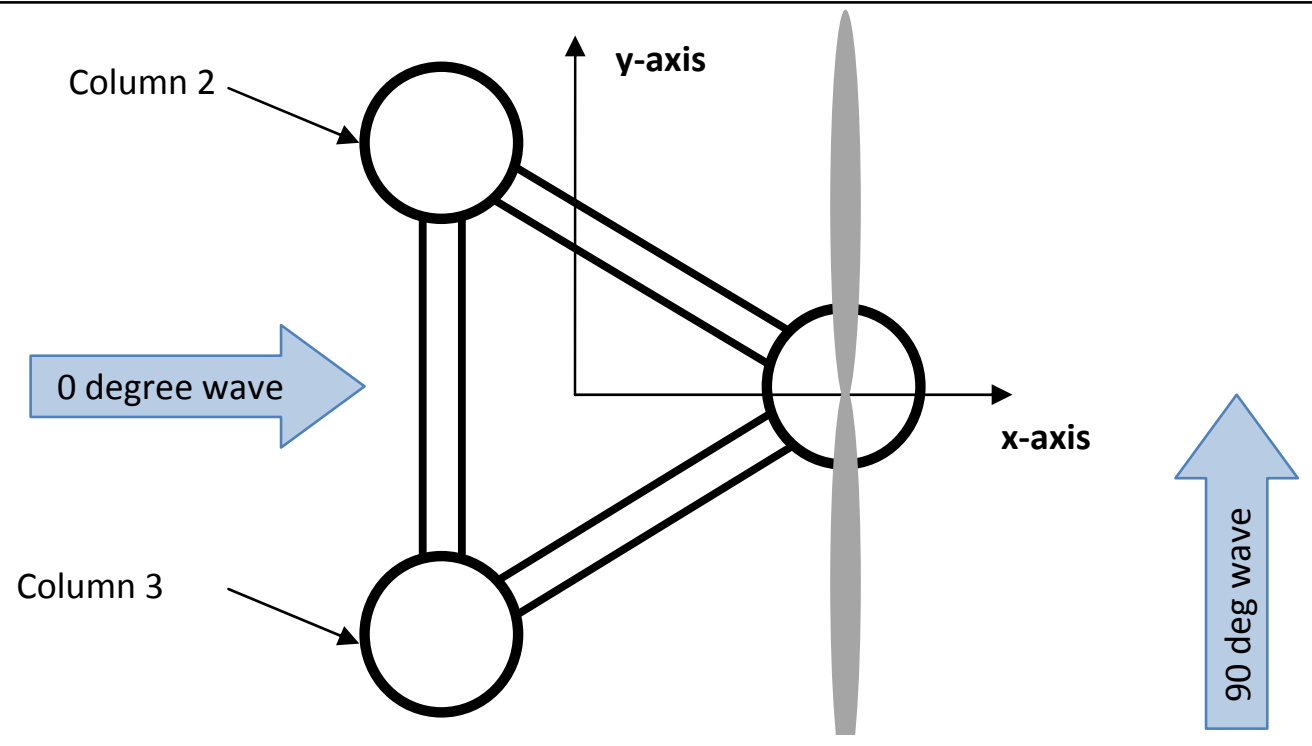

Figure 6: Definition of Coordinate System 


\subsection{Oscillating Water Column}

\subsubsection{Description}

Oscillating Water Columns harvest energy by transferring the energy due to oscillating wave elevation into pneumatic energy of a column of air inside a chamber.

On the WindFloat, an oscillating water column is fitted on each platform column without turbine (referred to as columns 2 and 3 ).

The chamber of the oscillating water column (OWC) is an annulus enclosed by the column shell and a cylindrical outer shell. Figure 7 shows the OWC chambers on columns 2 and 3.

The OWC is characterized by the following parameters:

- Diameter $\mathrm{D}_{\text {owc }}$ of the outer shell, which ranges between 14 and $20 \mathrm{~m}$ in the present analysis,

- Draft Towc of the outer shell, which ranges between 1 and $9 \mathrm{~m}$ herein,

- $\theta_{\mathrm{w} 1}$ and $\theta_{\mathrm{w} 2}$, the angles of the vertical walls that compartment the chamber. The angles are defined with respect to the $\mathrm{x}$-axis - as described in Section 2.1. For this analysis, $\theta_{\mathrm{w} 1}$ is set to 90 degrees and $\theta_{\mathrm{w} 2}$ is set to 180 degrees.

These geometric parameters are illustrated in Figure 8. $A_{C}$ is the total horizontal cross section of the OWC chamber. $V_{C}(t)$ is the time dependent volume of air in the chamber and $V_{c 0}$ is the volume of air when the system is at rest.

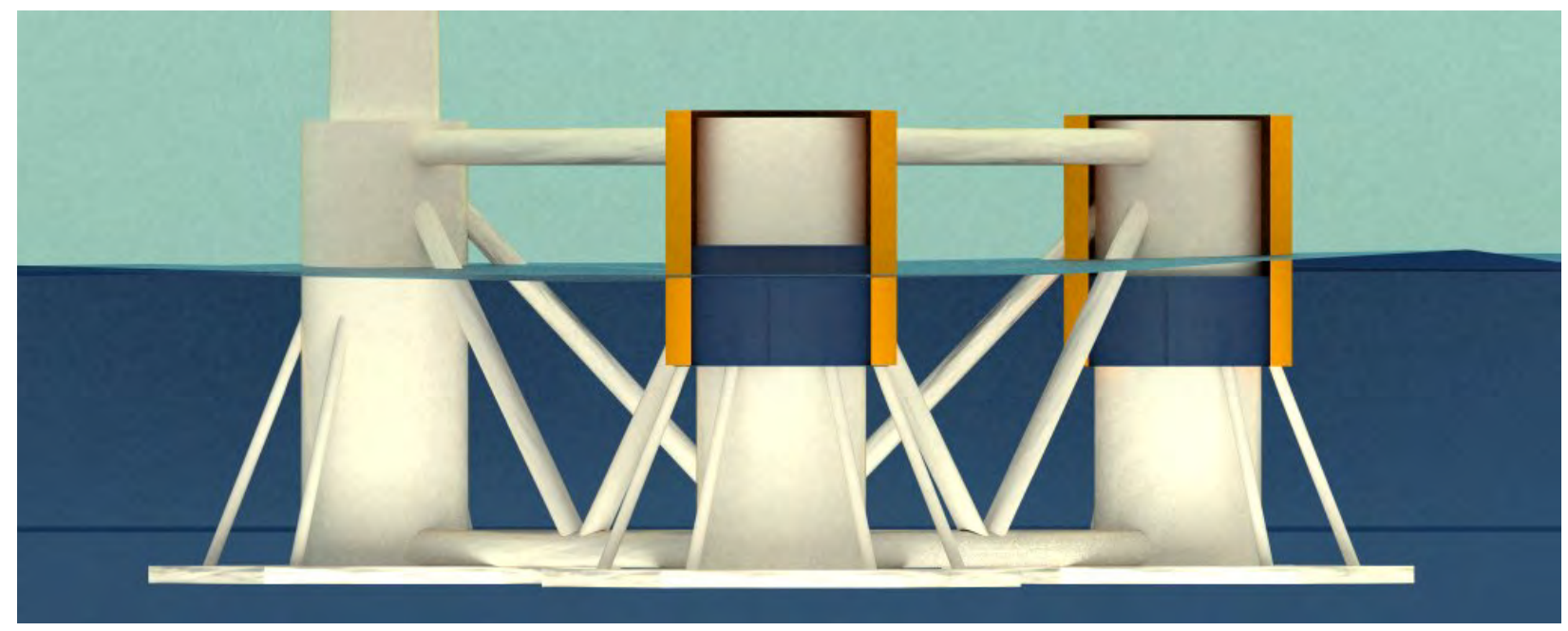

Figure 7: Side View of OWC Chambers on WindFloat 


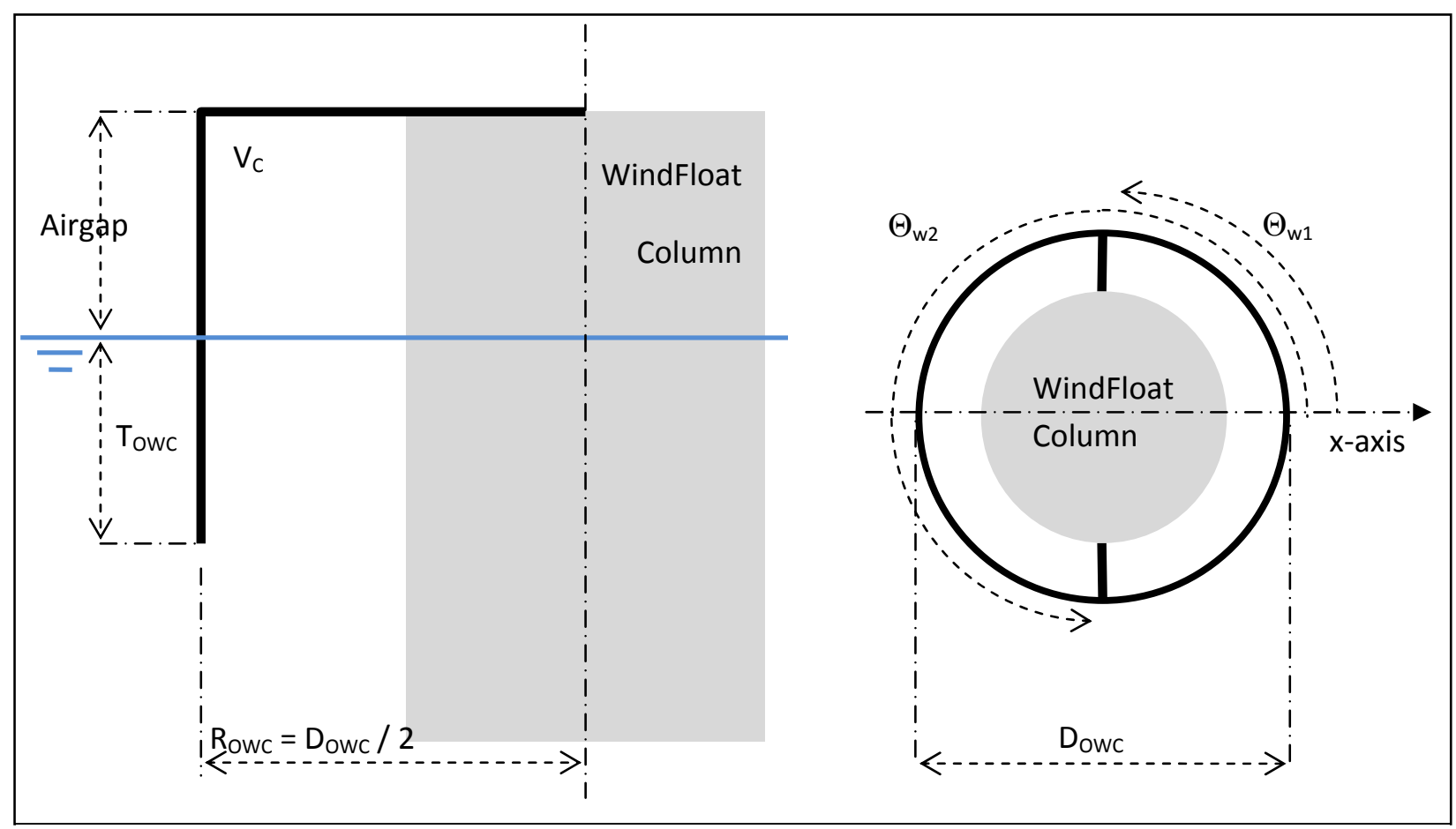

Figure 8: Cross Section of the OWC Chamber - Left: side view; Right: top view

The water elevation inside the chamber oscillates and drives the air in the upper section into a turbine shaft.

A Wells turbine will be used to extract the power from the air chamber. Other turbines such as airimpulse turbines have been considered for such applications in recent studies. But the Wells turbine is a low pressure air turbine with symmetrical airfoils which rotates in the same direction regardless of the direction of the incident airflow. Prof. A.A. Wells developed this turbine specifically for directionchanging airflows such as the wave motion induced airflow in an OWC. Extensive research has been done on the behavior and design of the Wells turbine. Some details can be found in Gato \& Falcăo [5] and Ragunathan [10]. It is used at a number of OWC power plants worldwide, including at the LIMPET $500 \mathrm{~kW}$ plant on the Island of Islay, in Scotland and at the European OWC Wave Power Plant, on the Island of Pico in the Azores.

The Wells turbine rotates at constant speed $\Omega$ and is defined by geometric properties, such as:

- $\quad$ Solidity S, the total blade area to turbine swept area ratio

- $\quad$ Radius $\mathrm{R}$, the outer radius of the blades

- $\quad$ Swept blade area $A_{T}$

The analysis of the turbine behavior and efficiency is outside of the scope of this report. Instead, characteristics of existing turbines will be used to assess the effectiveness of the design. 


\subsubsection{Theory}

The purpose of this analysis is to develop the numerical tools to model and analyze various configurations of the WindWaveFloat OWC. The turbine characteristics are fixed. Several combinations of draft and diameter are considered for the chamber to study the effect of chamber geometry on power output and global motion response. A single OWC is considered in the following analysis.

\section{- $\quad$ Global Hydrodynamic Response:}

The oscillating water columns on each side of the WindFloat are rigidly connected to the platform. The global response of the system can be predicted by single-body analysis of wave-induced motion.

This is done with WAMIT ${ }^{\circledR}$ diffraction-radiation program, which is based on linear potential theory. The thin walls of the Oscillating Water Column are represented in WAMIT using dipoles. The modeled geometry is represented in Figure 9 for a draft $T_{\text {owc }}$ of $3 \mathrm{~m}$ and an OWC diameter $\mathrm{D}_{\text {owc }}$ of $14 \mathrm{~m}$.

The setup and validation of the WAMIT model is discussed in Appendix. The same platform model is used as that described in Section 2.1. WAMIT provides the following results:

- Hydrodynamic Coefficients and Diffraction Forces,

- Response Amplitude Operators,

- Wave elevation coefficient in the chamber.
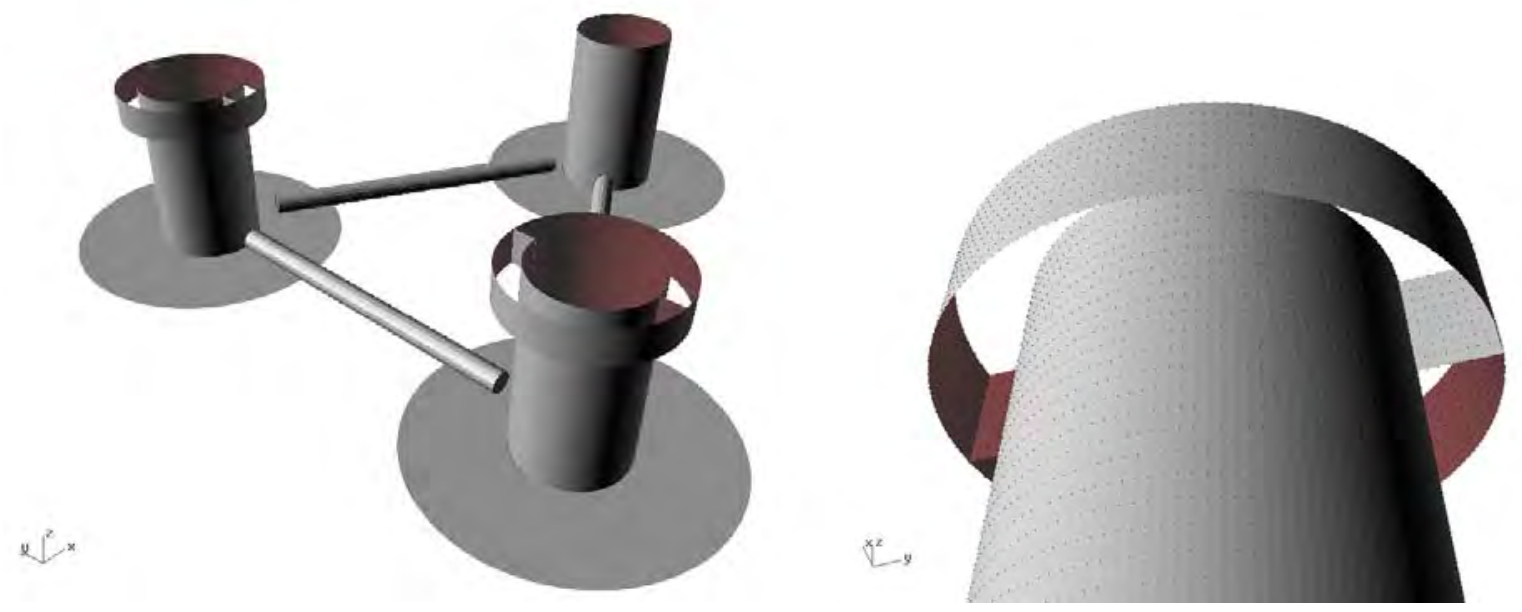

Figure 9 : Rhino3D Representation of WWF Submerged hull with OWC for WAMIT model

\section{- $\quad$ Power Output:}

It is shown in this section that the time dependent power converted by the turbine can be expressed as a quadratic function of volumetric flow rate through the OWC: 
$P=e(U) \frac{\alpha}{A_{T}} Q_{C}{ }^{2}$

where $\alpha$ and $A_{T}$ are turbine characteristics, $Q_{C}$ is the volumetric flow of air outside the OWC chamber and $e$ is the efficiency of the turbine which varies with the incident axial flow velocity $U$.

The airflow in the OWC and turbine chambers is assumed incompressible. This assumption is possible in the OWC chamber due to the relatively slow variations of volume. In a recent analysis Martins-Rivas [7] has included the effect of air compressibility. He remarks that it results in a loss of extracted power.

Losses in the Turbine-Generator system - including mechanical losses and electrical losses - are also neglected.

The power extracted by the turbine is the product of the rate of mass flux of air through the turbine by the work per unit mass of air provided to the turbine:

$P=\frac{d\left(\rho_{\text {air }} V\right)}{d t} \times \frac{\Delta p_{0}}{\rho_{\text {air }}}=\Delta p_{0} \times Q$

where $\Delta \mathrm{p}_{0}$ is the static pressure drop of the turbine and $Q=\frac{d V}{d t}$ is the volumetric air flow through the turbine.

Results by Gato and Falcăo [8], Ragunathan [10] and by Curran et al. [9] show that, when all turbine characteristics are fixed, the static pressure drop $\Delta p_{0}$ can be expressed as a linear function of $U$, the average axial velocity across the turbine cross section:

$\Delta p_{0}=\alpha U$ where $\alpha$ is a function of turbine solidity $S$, turbine rotational velocity $\Omega$, turbine radius $\mathrm{R}$, turbine loss coefficient and blade geometry.

$U$ is in turn related to the volumetric flow rate $Q$ :

$U=\frac{Q}{A_{T}}$

Applying the conservation of mass on an incompressible fluid and irrotational air flow between the chambers, the flow velocity in the turbine chamber $U$ can be related to the average flow velocity $U_{c}$ at the top of the OWC chamber:

$A_{C} U_{C}=A_{T} U$

Finally, $\mathrm{U}_{\mathrm{C}}$ can be expressed as a function of the volumetric flow rate in the OWC chamber: 


$$
U_{C}=\frac{1}{A_{C}} \frac{d V_{C}}{d t}
$$

The pneumatic extracted power can be approximated by the following formula:

$$
P=\frac{\alpha}{A_{T}}\left(\frac{d V_{C}}{d t}\right)^{2}=\frac{\alpha}{A_{T}}{\overline{Q_{C}}}^{2}
$$

Converted power is lower than pneumatic power. Typically the efficiency of a Wells turbine varies between 0.2 and 0.7 , depending on turbine characteristics. It can be assumed constant when the flow ratio $U / \Omega R$ is within an operational range. When flow ratio exceeds a certain value (typically 0.2 ), the efficiency of the turbine drops. If the flow ratio drops below a lower limit (typically 0.05), the turbine stalls and the efficiency is null. These are known limitations of current Wells turbines. As mentioned by Curran et al. [9], future design may incorporate control capabilities for the rotational speed and blade behavior to overcome this limitation. Guide vanes are also known to increase the efficiency of the turbine.

For this analysis, the efficiency is assumed to vary according to Figure 11 . The total converted power is equal to the product of $\mathrm{P}$ and turbine efficiency.

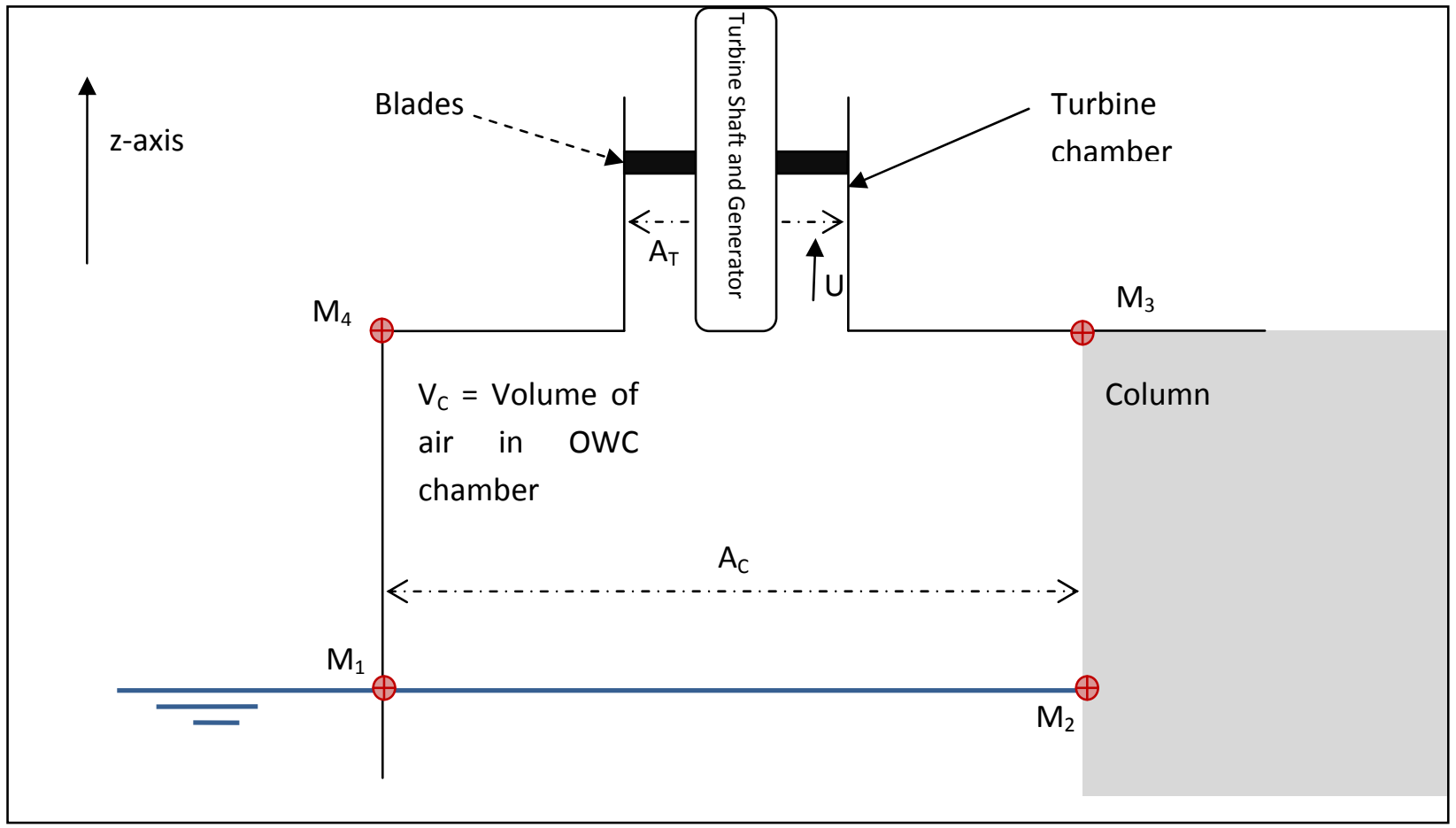

Figure 10 : Schematics of Power Extraction in OWC 


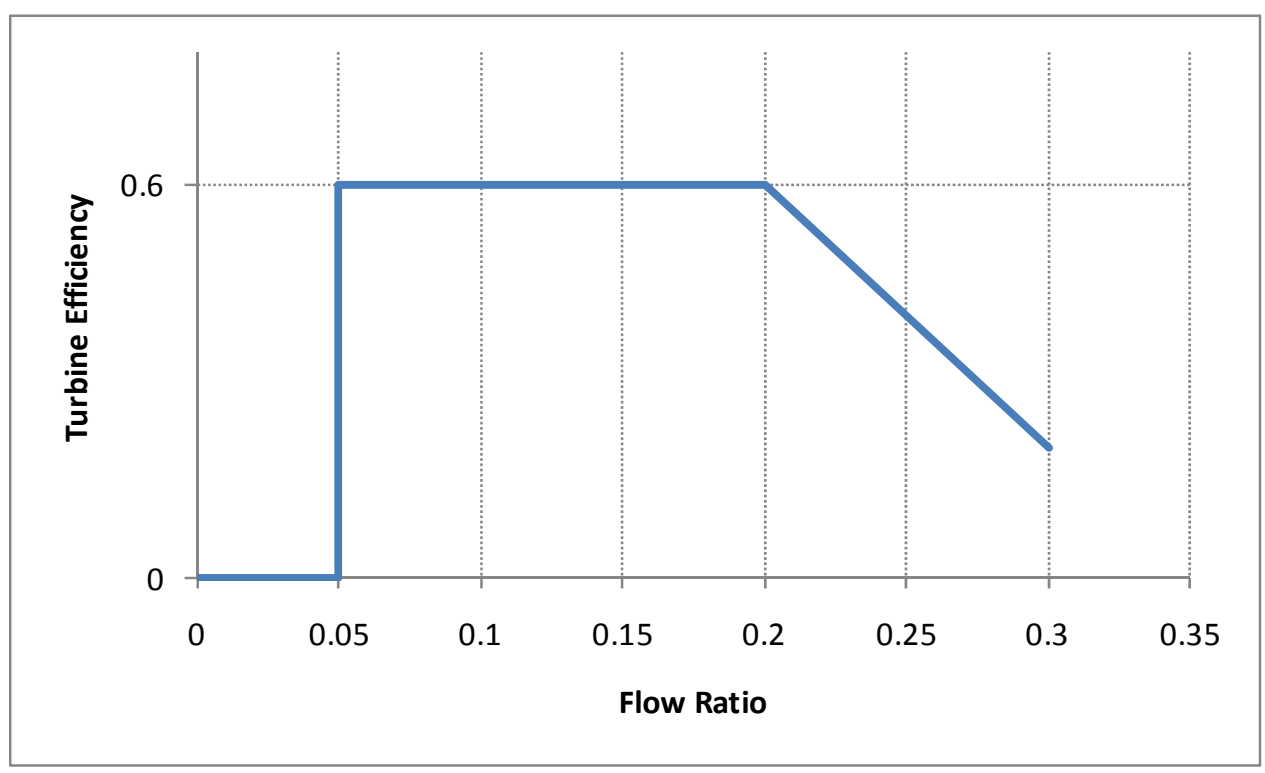

Figure 11 : Simplified model of turbine efficiency for OWC WindFloat

To compute the converted power, the variation of volume inside the OWC chamber is calculated.

The time dependent volume $V_{C}$ of the OWC chamber is:

$V_{C}(t)=V_{r a d}(t)-\int_{A_{C}} \eta(x, y, t) d S$

$\mathrm{V}_{\mathrm{rad}}$ is the time dependent enclosed volume of air assuming that the internal water surface is at rest. It is defined in 2D by the quadrilateral $\Gamma=M_{1} M_{2} M_{3} M_{4}$ in Figure 10. Since the OWC chamber is cylindrical, the enclosed volume $V_{C}$ is the integral of $A_{\Gamma}$, the surface area of $\Gamma$ from $\theta_{1}$ to $\theta_{2}$ :

$V_{\text {rad }}(t)=\int_{\theta_{1}}^{\theta_{2}} A_{\Gamma}(\theta, t) d \theta$

$A_{\Gamma}$ can be calculated for each cross section based on the RAOs of motion of the platform and a regular incident wave of amplitude $A$, period $T$ and phase $\varphi$. The RAOs are computed with WAMIT at the platform origin, as defined in Section 2.1. They are used to calculate the time dependent position of $M_{1}$, $M_{2}, M_{3}$ and $M_{4}$ at each angular position in the chamber, which yield $A_{\Gamma}$.

$\eta(x, y, t)=A \bar{\eta}(x, y) \cos (\omega t+\varphi+\phi(x, y))$ is the time dependent free surface wave elevation. The wave elevation coefficients $(\bar{\eta}, \phi)$ are obtained at a set of field points on the internal free surface using WAMIT. The effect of wave radiation and wave diffraction are included as well as the incident wave. In this approach, it is assumed that the action of the compressed air in the chamber on the internal water free surface in the OWC chamber is negligible. 
The volumetric flow rate $Q_{c}$ is the sum of a motion-induced term and of a free-surface term. Based on the above equations:

$$
Q_{C}=\frac{d V_{r a d}}{d t}-\int_{A_{C}} \dot{\eta} d S=\frac{V_{r a d}(t+d t)-V_{r a d}(t)}{d t}+A \omega \int_{A_{C}} \bar{\eta}(x, y) \sin (\omega t+\varphi+\phi(x, y)) d S
$$

The calculations of converted power $\mathrm{P}_{\text {converted }}$ and volumetric flow rate $\mathrm{Q}_{c}$ are implemented in Matlab. The WAMIT RAOs and wave elevation coefficients are input, as well as turbine parameter $\alpha$, OWC geometric parameters and incident wave properties. Numerical results are presented herein.

\subsection{Point Energy Absorber}

The point absorbers are modeled with Orcaflex and WAMIT. The following paragraph gives an overview of the numerical models used for the two generic designs.

\subsubsection{Single Device (SWEDE)}

The single device SWEDE is a spherical floater installed in the center of the WindFloat platform. The floater is attached to the column of the WindFloat by using three lines representing the power take-off system (unknown at this stage). Figure 12 presents the configuration of the SWEDE model. The sphere is colored in orange, and the three lines are colored in black.

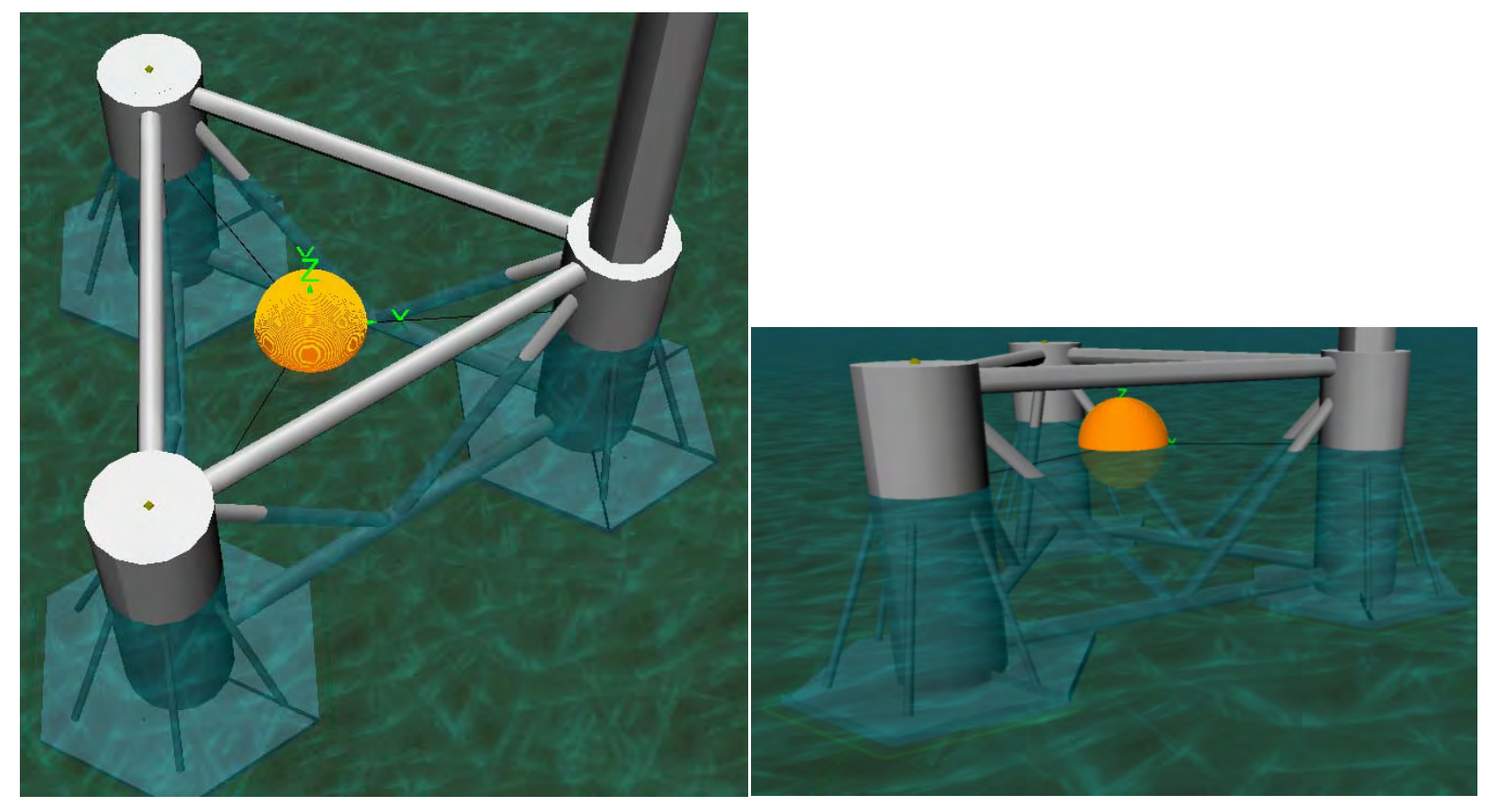

Figure 12 a, b: The SWEDE viewed from the top and the side. 
The sphere is modeled as a series of 100 co-axial cylinders mounted end-to-end along the local z-axis, with gradually increasing or diminishing diameters. The characteristics of the spherical floater are given in Table 4.

Table 4: Characteristics of the SWEDE

\begin{tabular}{|l|rc|}
\hline Sphere radius & 5.00 & $\mathrm{~m}$ \\
\hline Sphere draft & 5.00 & $\mathrm{~m}$ \\
\hline Sphere mass/displacement & 261.80 & tonnes \\
\hline Sphere wetted volume & 261.80 & $\mathrm{~m}^{3}$ \\
\hline Lines' pretension & 2,337 & $\mathrm{kN}$ \\
\hline Lines' stiffness & 1,500 & $\mathrm{kN} / \mathrm{m}$ \\
\hline
\end{tabular}

The lines are modeled as combined spring and independent damper units. The spring can take both compression and tension, and has a linear length-force relationship. The damper velocity-force relationship is also linear. The line characteristics, given in Table 4, are determined using the 100-year storm (see Table 3 ) to assure that the floater never hits the WindFloat columns or beams, and so that the tensions in the lines stay within their design values.

Hydrodynamic loads on the floaters are calculated using Morison's equation. Added mass and drag forces are applied only to those parts of the floater which are in the water at the time for which the force is calculated. The six degree-of-freedom added-mass coefficients, damping coefficients, and waveexciting loads of the floater are obtained with WAMIT using a single body analysis. Hydrodynamic coupling is neglected.

Regular sinusoidal waves of amplitudes $1 \mathrm{~m}, 3 \mathrm{~m}$, and $5 \mathrm{~m}$ are chosen. The period of the waves is varied from $2 \mathrm{~s}$ to $15 \mathrm{~s}$, and the damping of the lines is varied from 0 to $1,000 \mathrm{kN} . \mathrm{s} / \mathrm{m}$. Preliminary results are presented in section 3.3.1.

\subsubsection{Multiple Devices (Vertically constrained)}

The Multiple Devices concept is composed of three cylindrical floaters installed under the deck of the WindFloat platform. Each floater is attached to the beams of the WindFloat by using two lines representing the power take-off system (unknown at this stage but probably in the linear generator category). Figure 13 presents the configuration of the Multiple Devices model. The cylinders are colored in orange, and the six lines are colored in blue. 


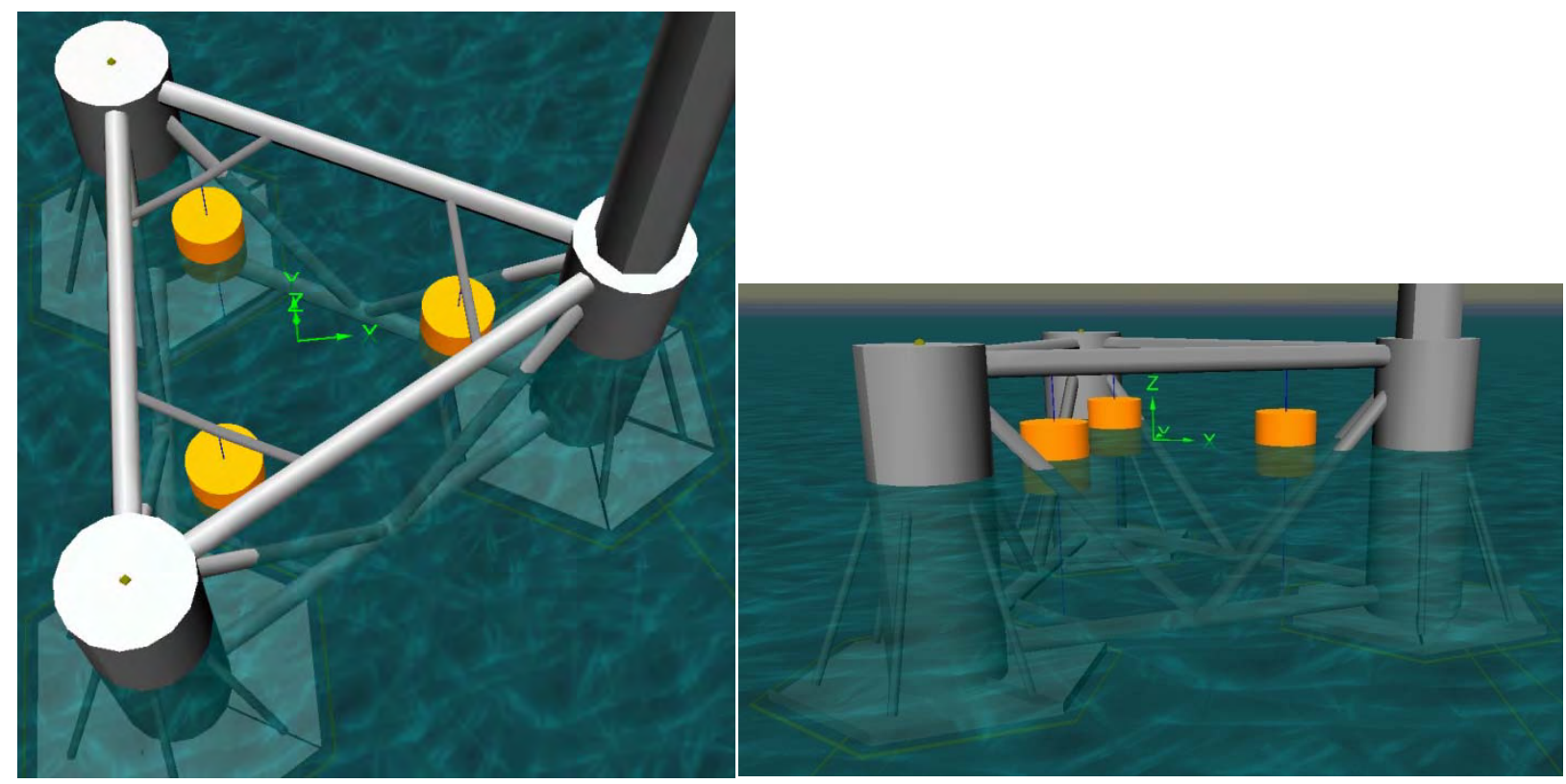

Figure 13a, b: The Multiple Devices viewed from the top and the side.

Each cylindrical floater is modeled as an axi-symmetric buoy whose z-axis is normally vertical. In reality, the cylinders are supposed to be guided vertically inside of the fixed part of the power take-off system. Therefore, the motion of the floaters is constrained in the vertical heave direction. This is achieved by inserting an infinite mass moment of inertia around the $x, y$, and $z$ local axes of each floater to set the angular motions to zero ( roll, pitch and yaw), and by setting all but the heave wave loads to zero. To provide a basis for comparative purposes, the wetted volume of the SWEDE is equal to the combined wetted volume of the three cylinders. The characteristics of the cylindrical floaters are given in Table 5.

Table 5: Characteristics of the cylindrical floaters

\begin{tabular}{|l|rc|}
\hline Cylinder radius & 3.00 & $\mathrm{~m}$ \\
\hline Cylinder height & 6.00 & $\mathrm{~m}$ \\
\hline Cylinder draft & 3.00 & $\mathrm{~m}$ \\
\hline Cylinder mass/displacement & 84.82 & tonnes \\
\hline Cylinder wetted volume & 84.82 & $\mathrm{~m}^{3}$ \\
\hline Lines' pretension & 500 & $\mathrm{kN}$ \\
\hline Lines' stiffness & 500 & $\mathrm{kN} / \mathrm{m}$ \\
\hline
\end{tabular}

Similar to the SWEDE, the lines are modeled as combined spring and independent damper units. The spring can take both compression and tension, and has a linear length-force relationship. The damper velocity-force relationship is also linear. The line characteristics, given in Table 5, are determined using the 100-year storm.

Similarly to the SWEDE, hydrodynamic loads on the floaters are calculated using Morison's equation. The six degree-of-freedom added-mass coefficients, damping coefficients, and wave-exciting loads of the floater are obtained with WAMIT. 
Regular sinusoidal waves of amplitudes of $1 \mathrm{~m}, 3 \mathrm{~m}$, and $5 \mathrm{~m}$ are chosen. The period of the waves is varied from $2 \mathrm{~s}$ to $15 \mathrm{~s}$, and the damping of the lines is varied from 0 to $1,000 \mathrm{kN} . \mathrm{s} / \mathrm{m}$. Preliminary results are presented in section 0 .

\subsection{Oscillating Plates}

The oscillating plates are also modeled with Orcaflex and WAMIT.

The Oscillating Plates concept consists of three rectangular flaps (flat stiffened vertical plates) hinged on the three top main beams of the WindFloat platform. The flaps oscillate back and forth as the waves hit them. The lower edge of each flap is attached to two lines representing the power take-off system (unknown at this stage but either in the hydraulic or electrical motor category) mounted on the WindFloat platform. Figure 14 presents the configuration of the Oscillating Plates model. The flaps are colored in orange, and the six lines are colored in blue. The hinge mechanism is not presented on the figure.
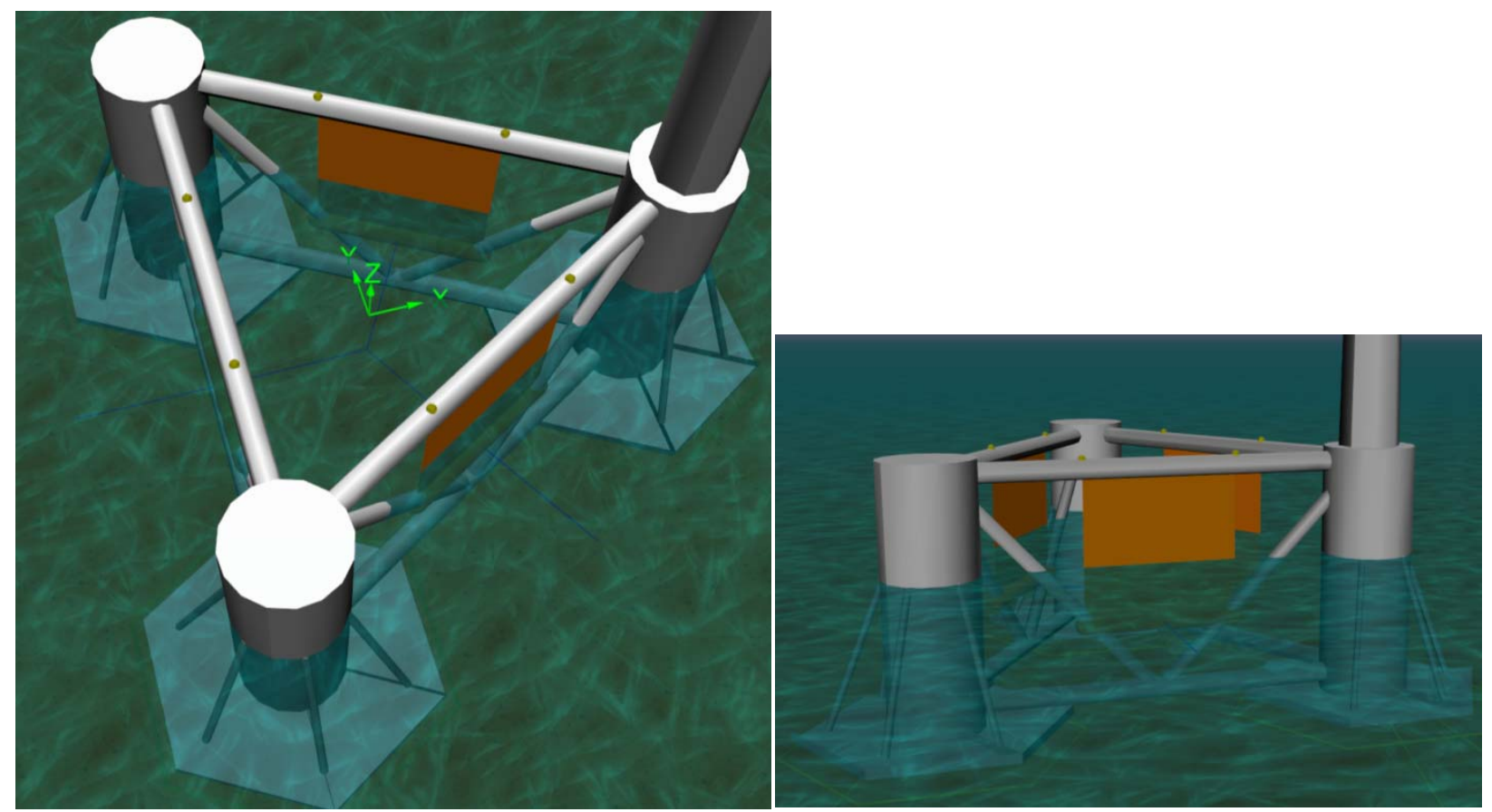

Figure 14a, b: The Oscillating Plates viewed from the top and the side.

The hinge mechanism is , modeled by connecting the flaps to line members attached to the WindFloat platform, with an infinite bending stiffness at the flap-line connection in all directions. 
Table 6: Characteristics of the flaps

\begin{tabular}{|l|rc|}
\hline Flap length & 16.00 & $\mathrm{~m}$ \\
\hline Flap width & 16.00 & $\mathrm{~m}$ \\
\hline Flap thickness & 0.01 & $\mathrm{~m}$ \\
\hline Flap draft & 6.00 & $\mathrm{~m}$ \\
\hline Flap mass & 20.10 & tonnes \\
\hline Flap wetted volume & 0.96 & $\mathrm{~m}^{3}$ \\
\hline Lines' pretension & 250 & $\mathrm{kN}$ \\
\hline Lines' stiffness & 2,070 & $\mathrm{kN} / \mathrm{m}$ \\
\hline
\end{tabular}

Similarly, the six lines are modeled as combined spring and independent damper units. The spring can take both compression and tension, and has a linear length-force relationship. The damper velocityforce relationship is also linear. The line characteristics, given in Table 6, are determined using the 100year storm, by assuring that the flap maximum angle stays within its design range.

Similarly to the other WWF concepts, hydrodynamic loads on the flaps are calculated using Morison's equation. The six degree-of-freedom added-mass coefficients, damping coefficients, and wave-exciting loads of the flaps are obtained with WAMIT dipole elements. The flaps are modeled in Orcaflex by using rectangular vessels instead of 6DOF floaters. Vessels are in general more challenging to model but offer a higher accuracy.

Regular sinusoidal waves of amplitudes of $1 \mathrm{~m}, 2 \mathrm{~m}$, and $3 \mathrm{~m}$ are chosen. The period of the waves is varied from $4 \mathrm{~s}$ to $17 \mathrm{~s}$, and the damping of the lines is varied from 0 to $1,000 \mathrm{kN} . \mathrm{s} / \mathrm{m}$. Preliminary results are presented in section 3.4 .

\section{Preliminary results}

\subsection{Base Case}

The dynamic characteristics of the two models are compared in this section.

The periods of resonance in the 6 degrees of freedom are predicted using the following formula: $T_{i}=2 \pi \sqrt{\frac{M_{i}+A_{i, i}}{C_{i, i}+K_{i}}}$

where $M$ is the $6 \times 6$ mass matrix, $A$ is the $6 \times 6$ added mass matrix computed by WAMIT, $C$ is the hydrostatic stiffness matrix also computed by WAMIT and $\mathrm{K}$ is the equivalent mooring stiffness. Table 7 summarizes the results. Decay tests were carried out in Orcaflex to verify the periods of resonance. The equivalent mooring stiffness was adjusted in WAMIT so the two models have the same resonant frequencies in surge, sway and yaw. Since OrcaFlex uses the WAMIT calculated added mass and damping terms, it is inherent that the natural periods in the non-mooring dependent motions (heave, roll and pitch) are similar. 
Table 7: Periods of Resonance of the Base Case

\begin{tabular}{|l|c|c|c|c|c|c|}
\hline Direction of motion & Surge & Sway & Heave & Roll & Pitch & Yaw \\
\hline Period of resonance [s] & 108.6 & 135.7 & 19.9 & 43.3 & 43.2 & 71.1 \\
\hline
\end{tabular}

The wave-induced behavior of the base case is captured by the Response Amplitude Operators (RAOs). Only the 0 degree heading waves are considered. Due to the symmetries of the system, the RAOs in sway, roll and yaw are null at this heading.

Figure 15 to Figure 17 represent the RAOs in surge, heave and pitch for the two models. Some discrepancies appear. They are likely due to the presence of non-linearity in the Orcaflex model which cannot be accounted for in the WAMIT model.

The pitch response is low, with less than 0.6 degree per meter of wave across the wave range.

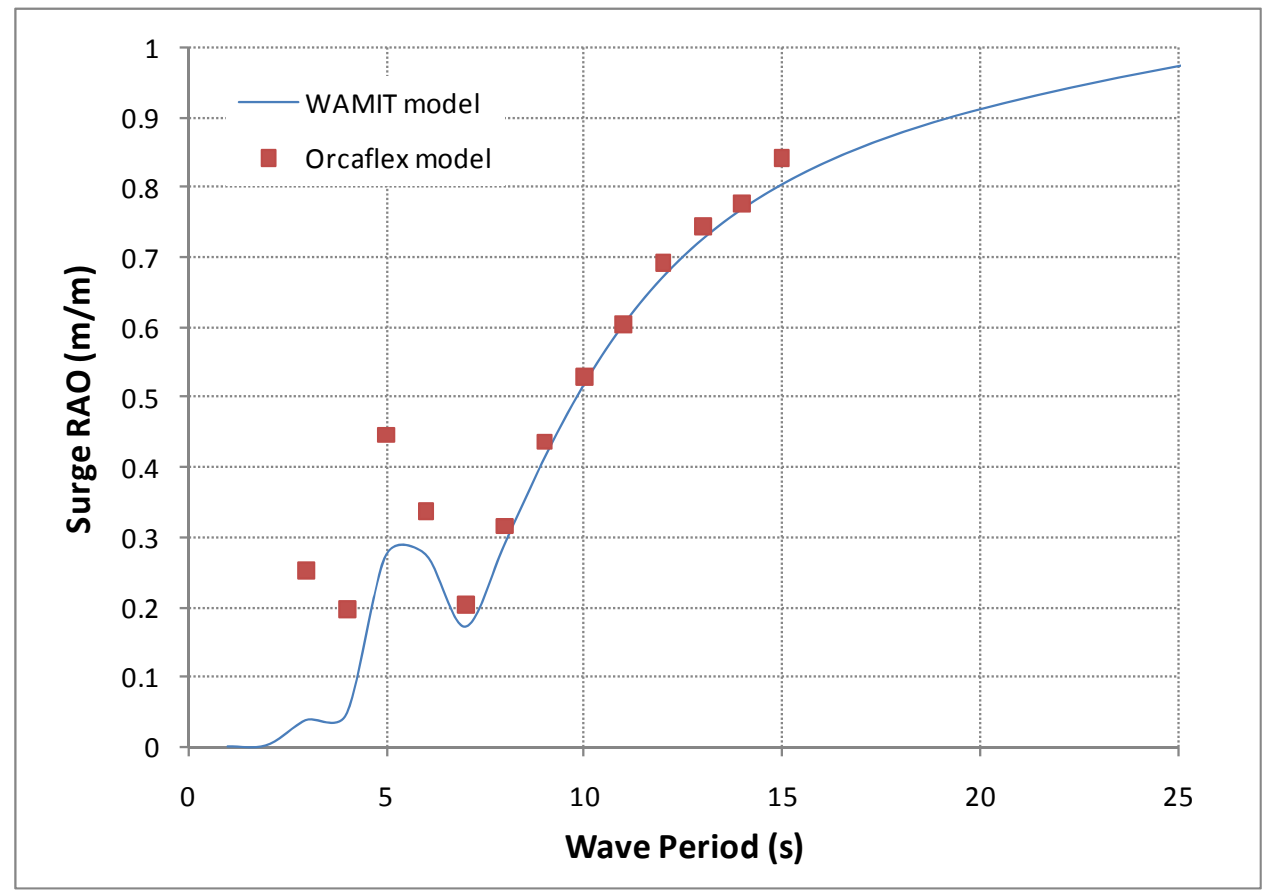

Figure 15 : Comparison of Surge RAO for 0 degree heading waves in Base Case 


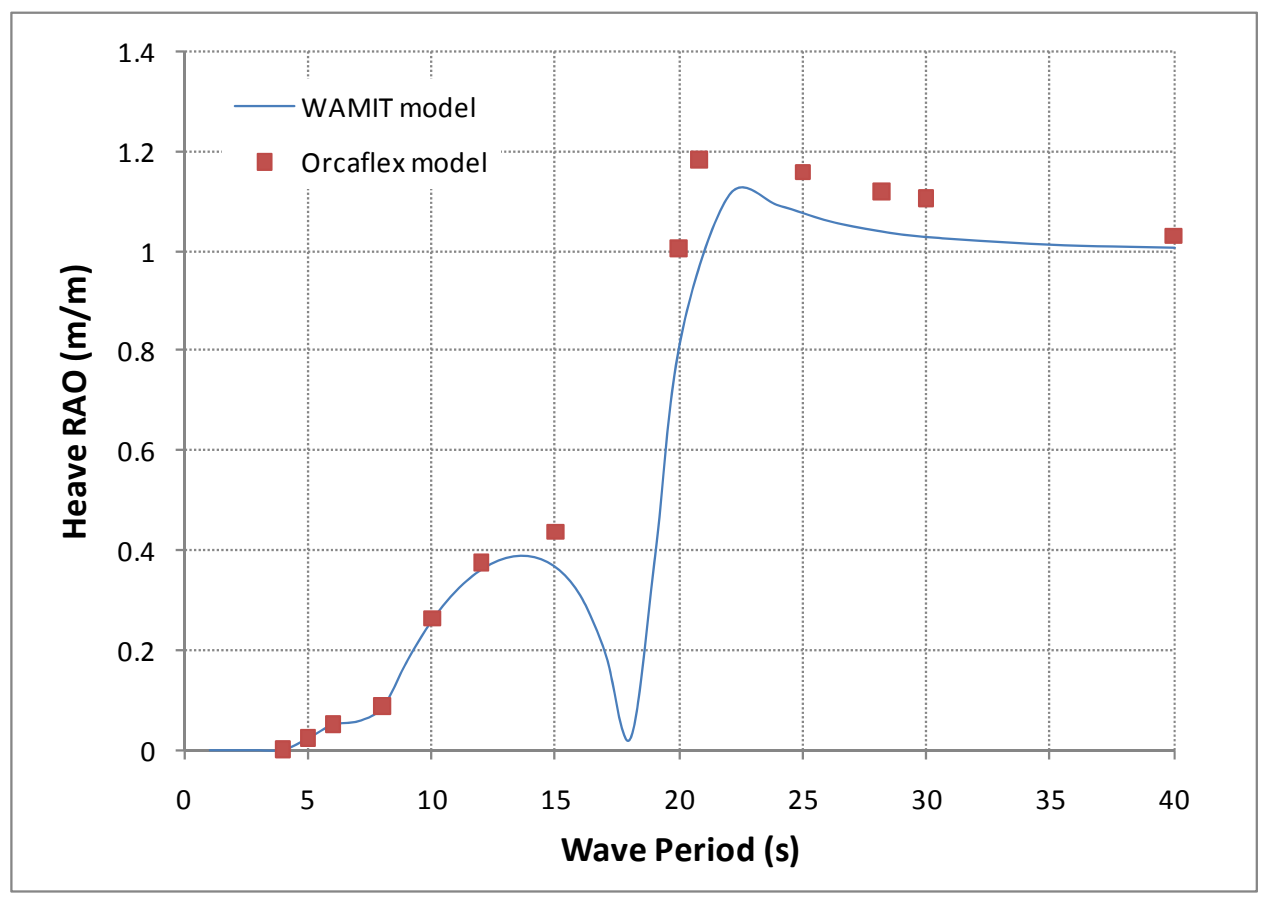

Figure 16: Comparison of Heave RAO for 0 degree heading waves in Base Case

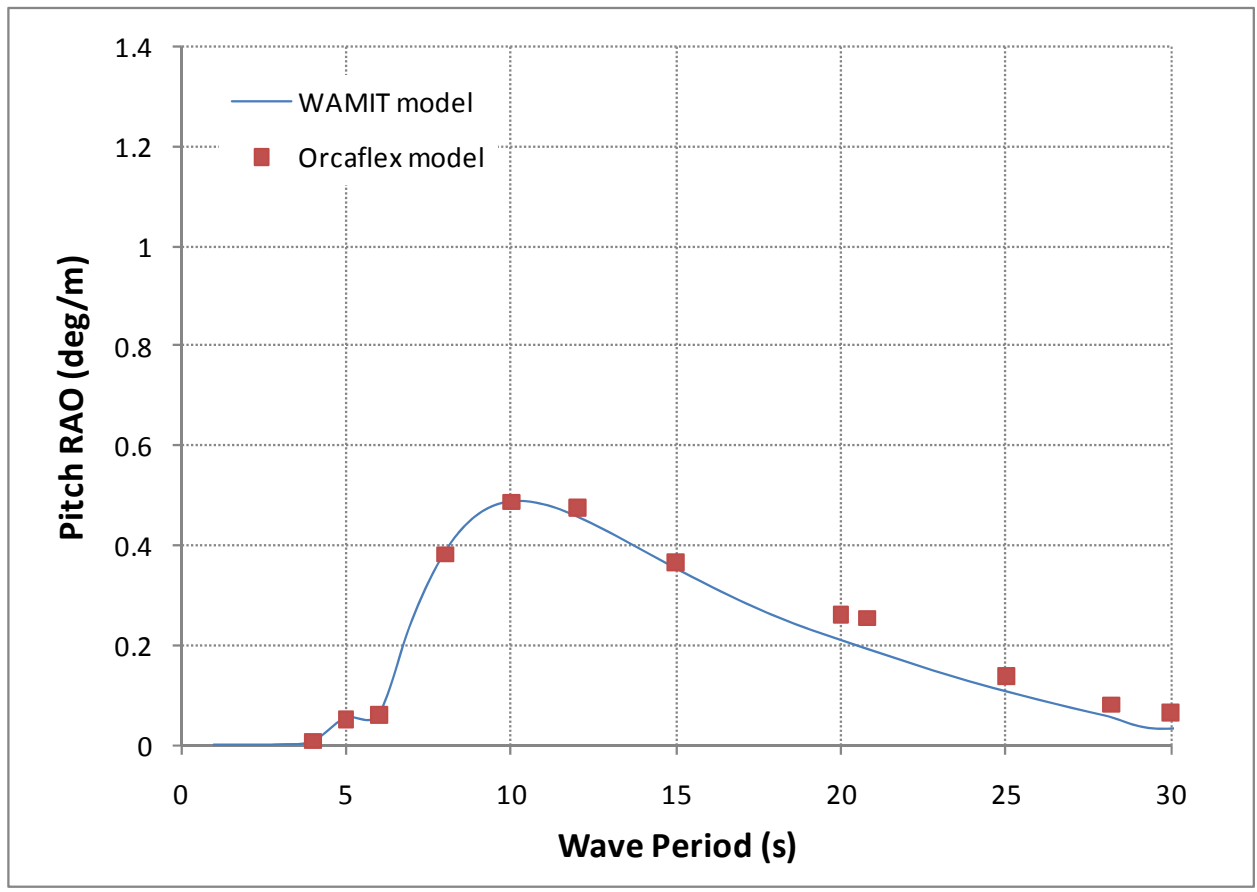

Figure 17: Comparison of Pitch RAO for 0 degree heading waves in Base Case 


\subsection{Oscillating Water Column}

\subsubsection{Hydrodynamic Characteristics}

The water column inside the chamber acts as a forced oscillating body with one degree of freedom. In a first approach, its motion verifies the equation of a spring-mass-damper system:

$M \ddot{\eta}+B \dot{\eta}+K \eta=F(t)$

In this case, $M=\rho_{w} A_{C} T_{\text {OWC }}$ is the mass of the water column at rest, B the damping and $K=\rho_{w} g A_{C}$ is the stiffness of the internal free surface.

Using this simple model, the natural period of the OWC depends on the OWC draft Towc to gravitational acceleration ratio:

$T=2 \pi \sqrt{\frac{M}{K}}=2 \pi \sqrt{\frac{T_{O W C}}{g}}$

Figure 18 represents the maximum wave elevation per meter of incident wave height inside the cylindrical chamber as a function of the wave period. This wave elevation RAO is obtained by computing the wave elevation of the free surface with WAMIT. It is compared for OWC drafts between 1 and $9 \mathrm{~m}$. In this case, the diameter of the OWC is fixed to $14 \mathrm{~m}$. The corresponding predicted resonant periods are provided in Table 8. Note that, at low periods, convergence issues might affect the results.

Table 8: Theoretical Periods of Resonance on OWC

\begin{tabular}{|l|c|c|c|c|c|}
\hline OWC Draft Towc $[\mathrm{m}]$ & 1 & 3 & 5 & 7 & 9 \\
\hline Period of Resonance $[\mathrm{s}]$ & 2.0 & 3.5 & 4.5 & 5.3 & 6.0 \\
\hline
\end{tabular}




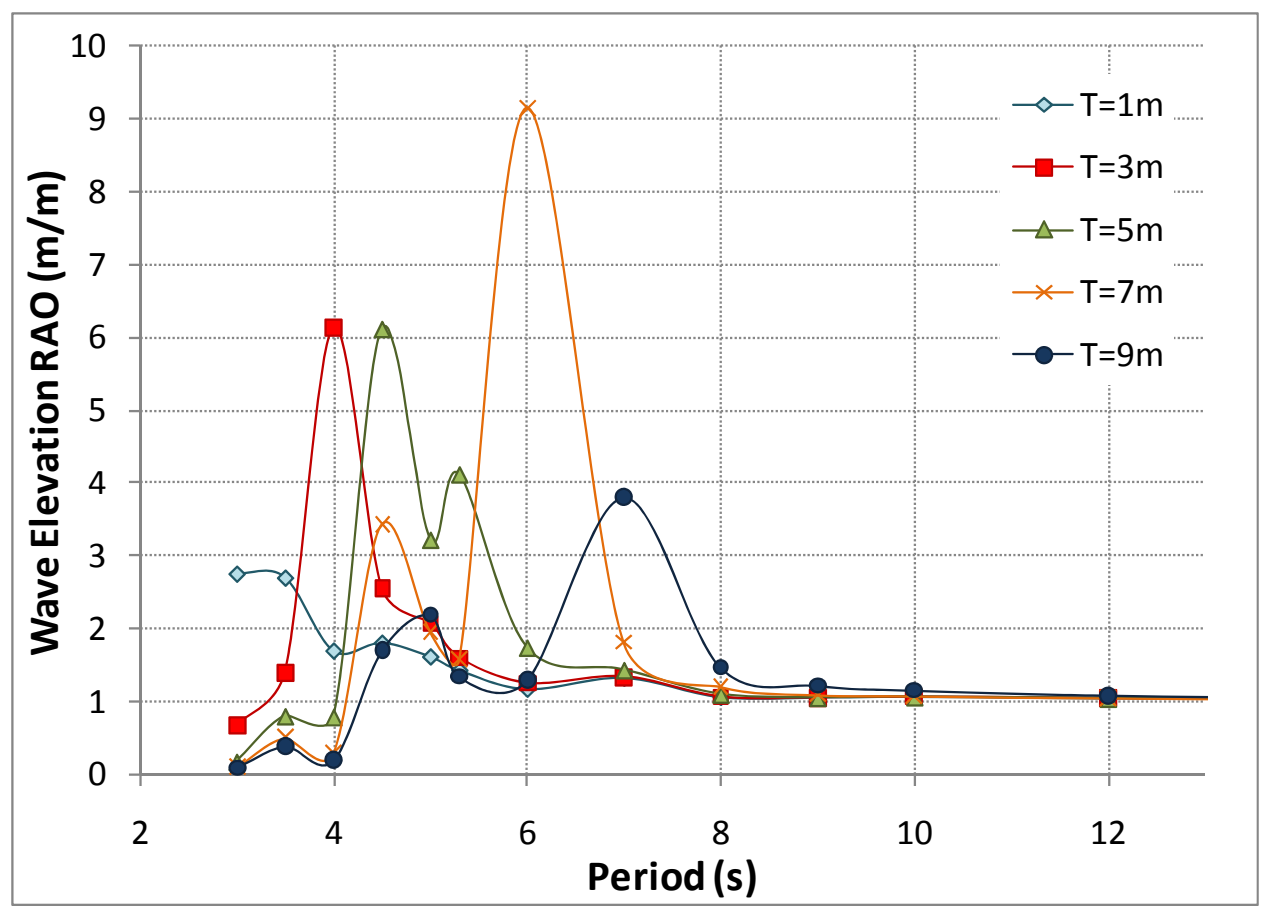

Figure 18 : Maximum Wave Elevation RAO in OWC Chamber for different OWC drafts

WAMIT predicts slightly larger periods than the theory in this case. However, theoretical values are based on the assumption that the wave elevation is uniform in the chamber. In reality, especially at small periods, local variations of the surface elevation are noticeable.

For instance, a secondary period of resonance can be observed in Figure 18. It is the standing wave resonance inside the column. With $D_{\text {owc }}=14 \mathrm{~m}$, the corresponding resonant wavelength is $28 \mathrm{~m}$, which occurs at $T=4.2 \mathrm{~s}$ in deep water. To remain efficient at these low wave periods, it may be necessary to partition the OWC cylinder into smaller compartments using vertical walls.

Figure 19 to Figure 23 show the wave amplitude coefficient (amplitude of wave elevation per meter of incident wave) calculated by WAMIT on the $40 \mathrm{~m} \times 40 \mathrm{~m}$ free surface around the WindFloat at the computed period of resonance. The horizontal axis represents the $x$-axis, the vertical axis is the $y$-axis. The WindFloat columns are symbolized by dark blue or white circles. 


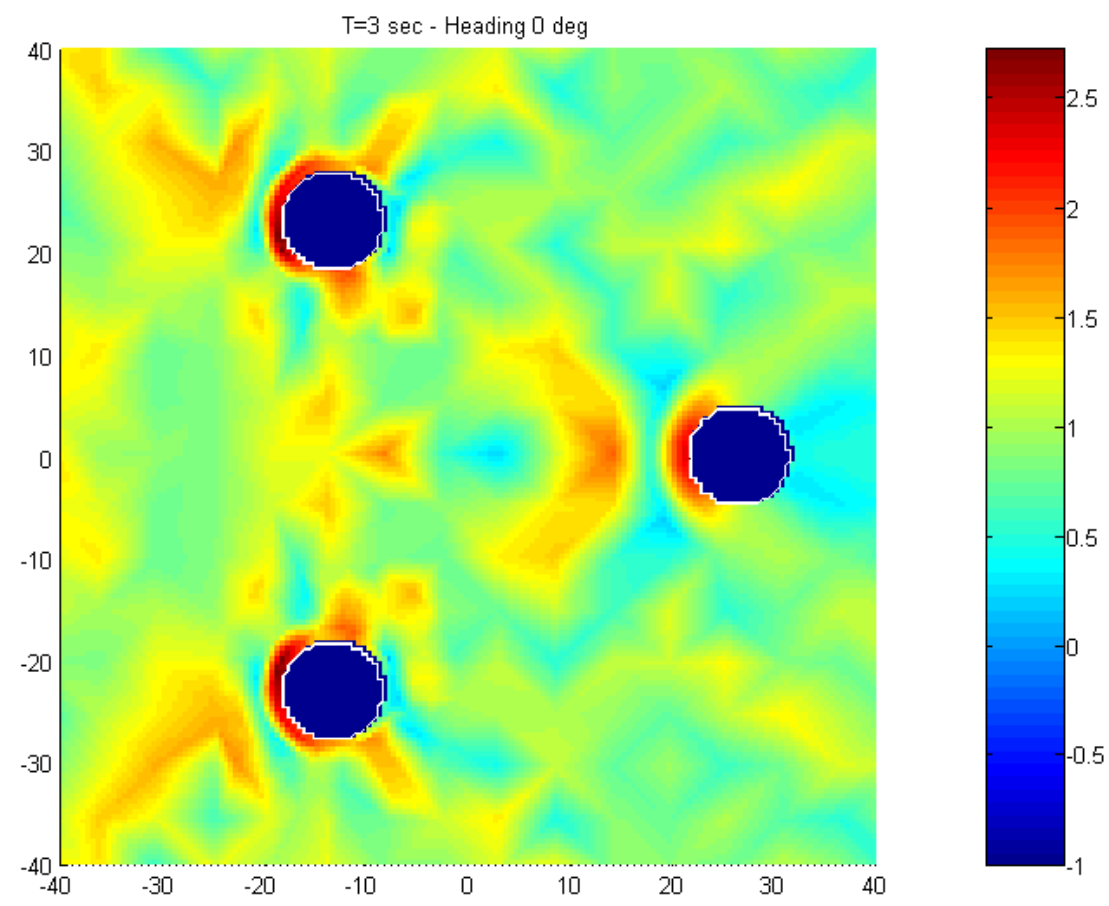

Figure 19: Map of Wave Amplitude Coeff. [m/m] in wave field for $1 \mathrm{~m}$ draft $0 W C$ at $T=3 \mathrm{~s}$

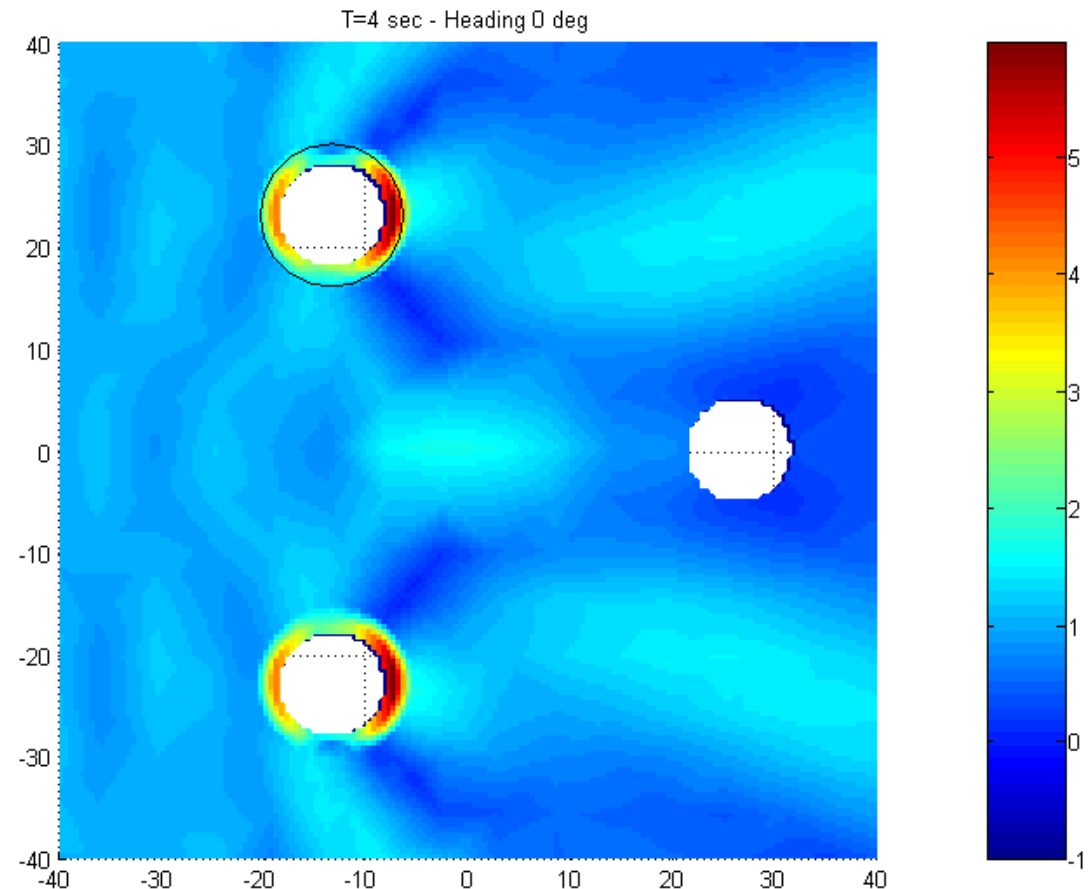

Figure 20: Map of Wave Amplitude Coeff. $[\mathrm{m} / \mathrm{m}]$ in wave field for $3 \mathrm{~m}$ draft $\mathrm{OWC}$ at $\mathrm{T}=4 \mathrm{~s}$ 


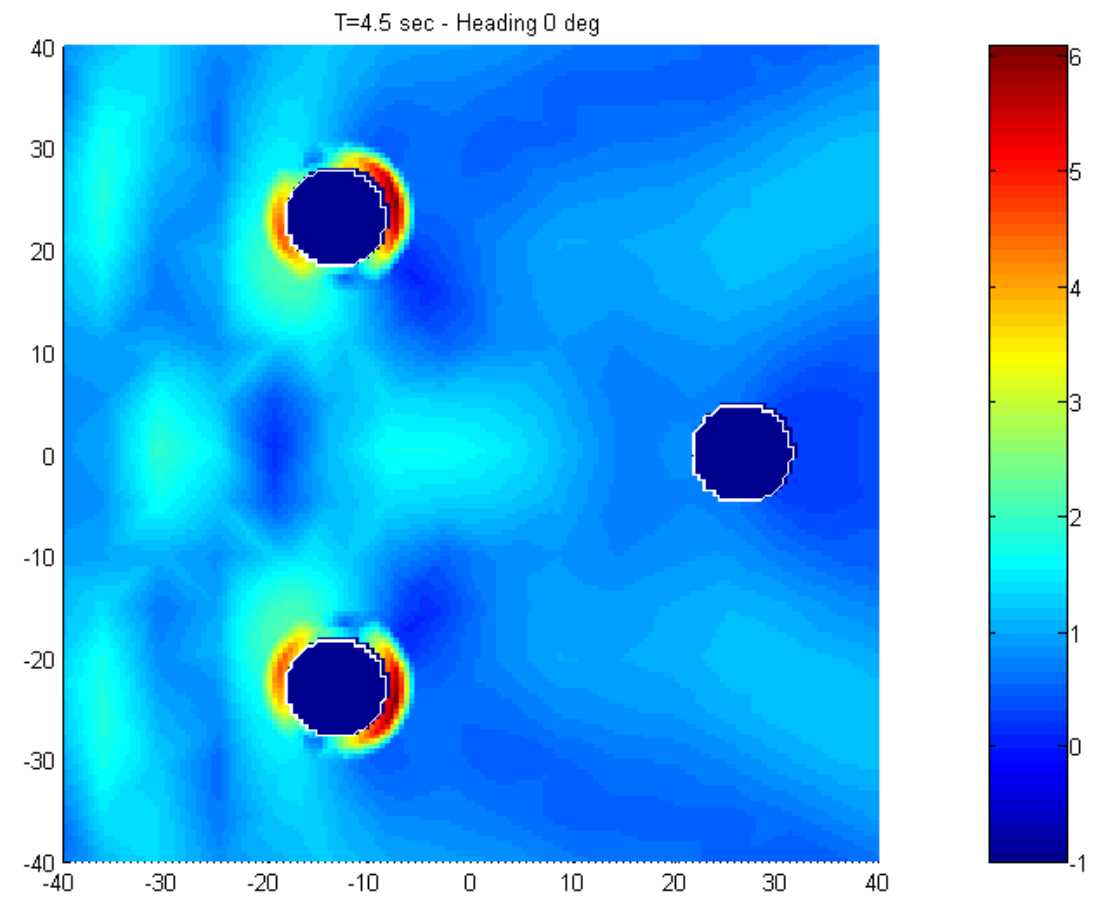

Figure 21: Map of Wave Amplitude Coeff. $[\mathrm{m} / \mathrm{m}]$ in wave field for $5 \mathrm{~m}$ draft $0 W C$ at $\mathrm{T}=4.5 \mathrm{~s}$

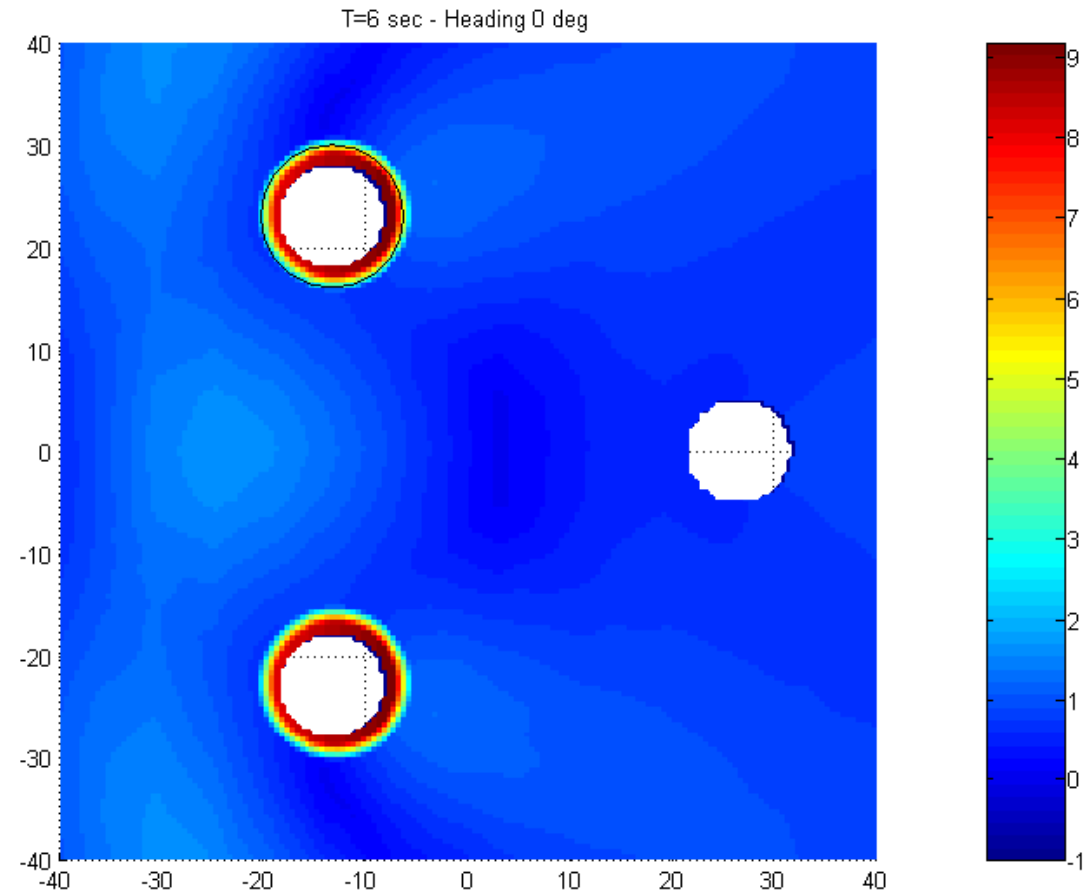

Figure 22: Map of Wave Amplitude Coeff. $[\mathrm{m} / \mathrm{m}]$ in wave field for $7 \mathrm{~m}$ draft $0 W C$ at $T=6 \mathrm{~s}$ 


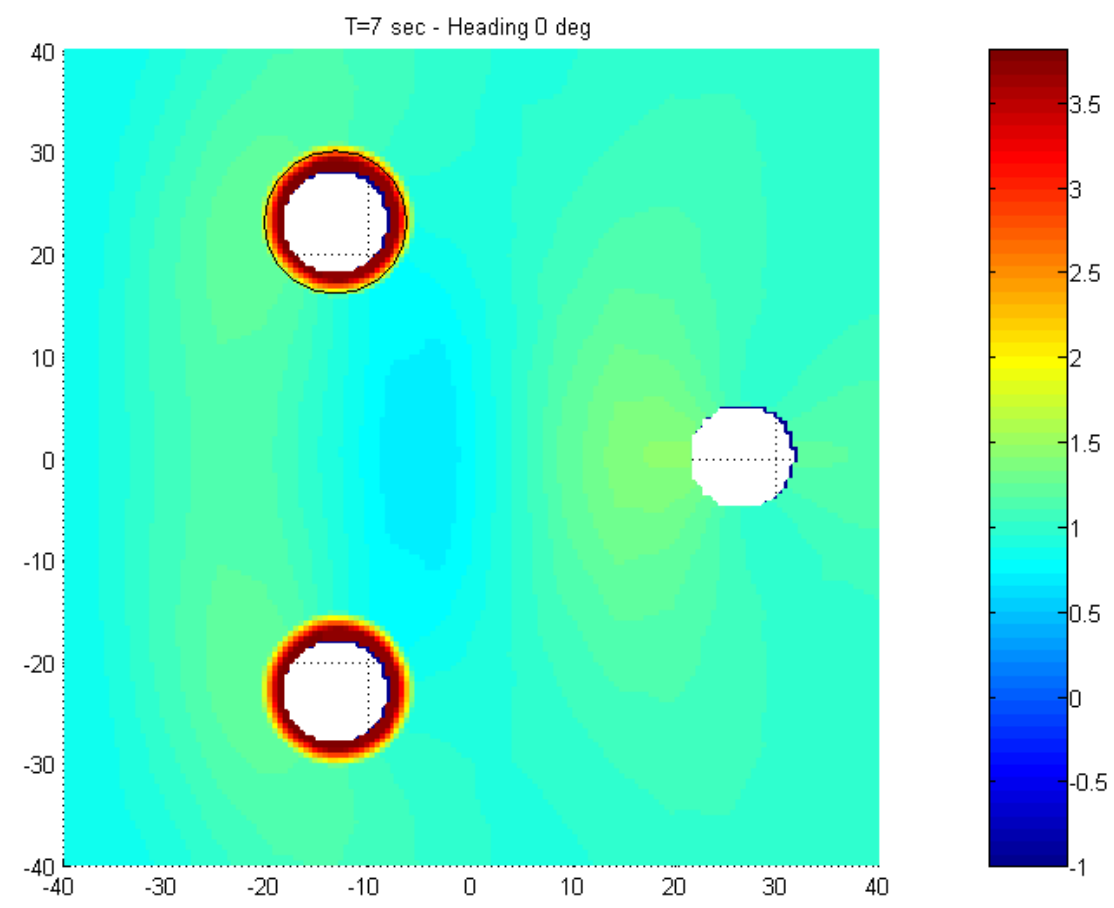

Figure 23: Map of Wave Amplitude Coeff. $[\mathrm{m} / \mathrm{m}]$ in wave field for $9 \mathrm{~m}$ draft $0 W C$ at $T=7 \mathrm{~s}$

\subsubsection{Effect on Platform Motions}

Figure 24 to Figure 26 compares the global RAOs of motion of the OWC WindFloat with the RAOs of the base case for various OWC drafts. The effect of OWC diameter is studied in Figure 27 to Figure 29. This analysis is made without internal walls in the OWC.

The mass matrix, equivalent damping matrix and equivalent stiffness matrix are identical to those in the base case. In reality, viscous damping is likely to increase due to the presence of the OWC walls. This should be confirmed with model tests. The mass distribution would also change. The present results only capture the variations of geometry-dependent radiation-diffraction.

For an OWC diameter of $14 \mathrm{~m}$, the effect of the OWC on the global response of the platform is not significant, regardless of the OWC draft. When the diameter varies in Figure 27 to Figure 29, the OWC affects the amplitude of surge motion at low wave periods. It also introduces a small resonance at the natural period of the OWC in heave and pitch. Such resonance only becomes apparent when the OWC diameter is large. 

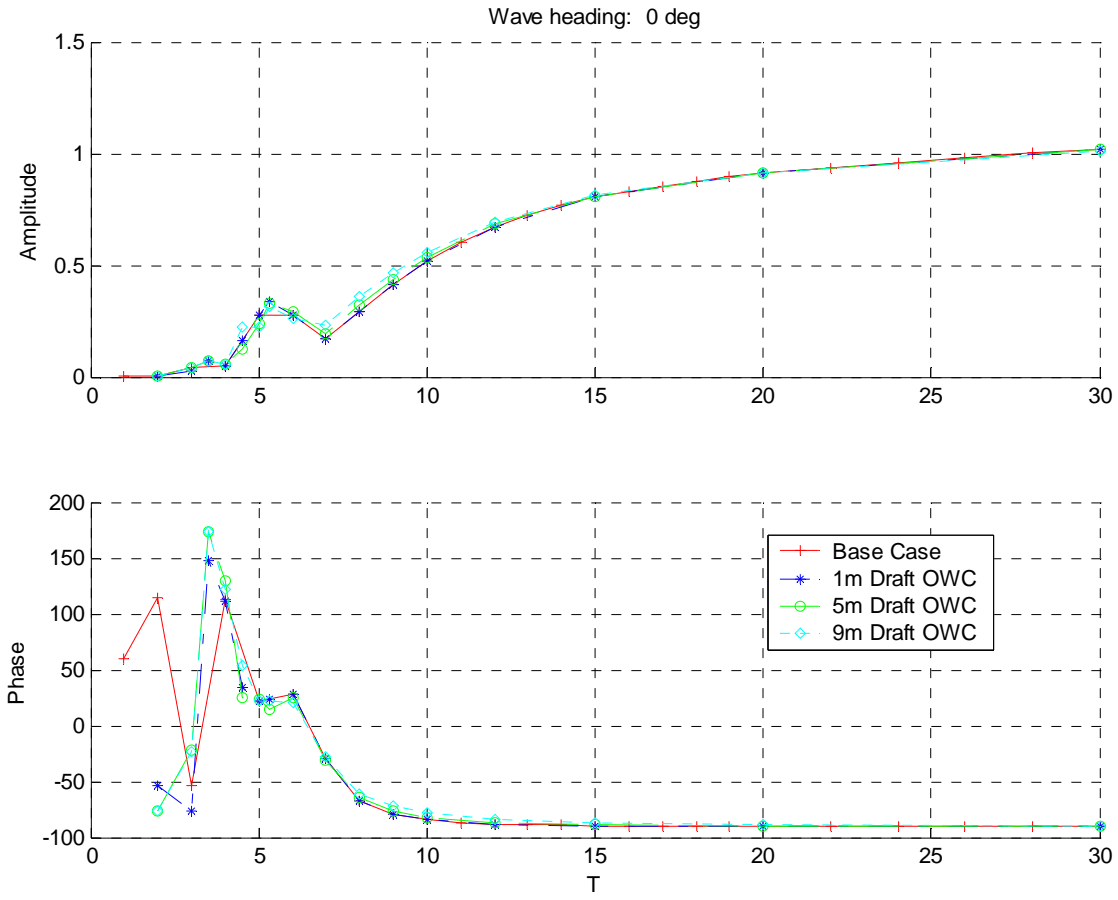

RAO in direction 1

Figure 24: Surge RAO (amplitude in $\mathrm{m} / \mathrm{m}$, phase in deg) of OWC WindFloat for various drafts with $\mathrm{D}_{\mathrm{owc}}=14 \mathrm{~m}$
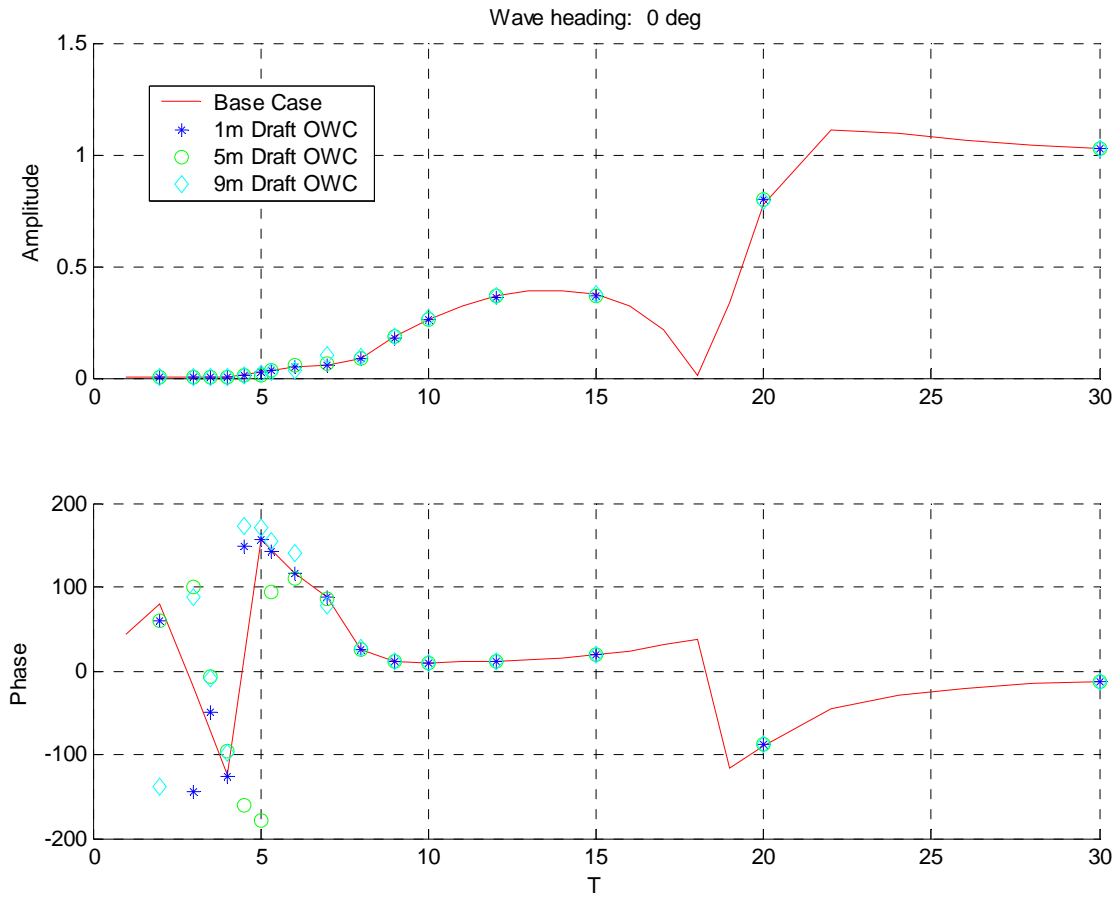

RAO in direction 3

Figure 25: Heave RAO (amplitude in $\mathrm{m} / \mathrm{m}$, phase in deg) of OWC WindFloat for various drafts with $\mathrm{D}_{\mathrm{owc}}=14 \mathrm{~m}$ 

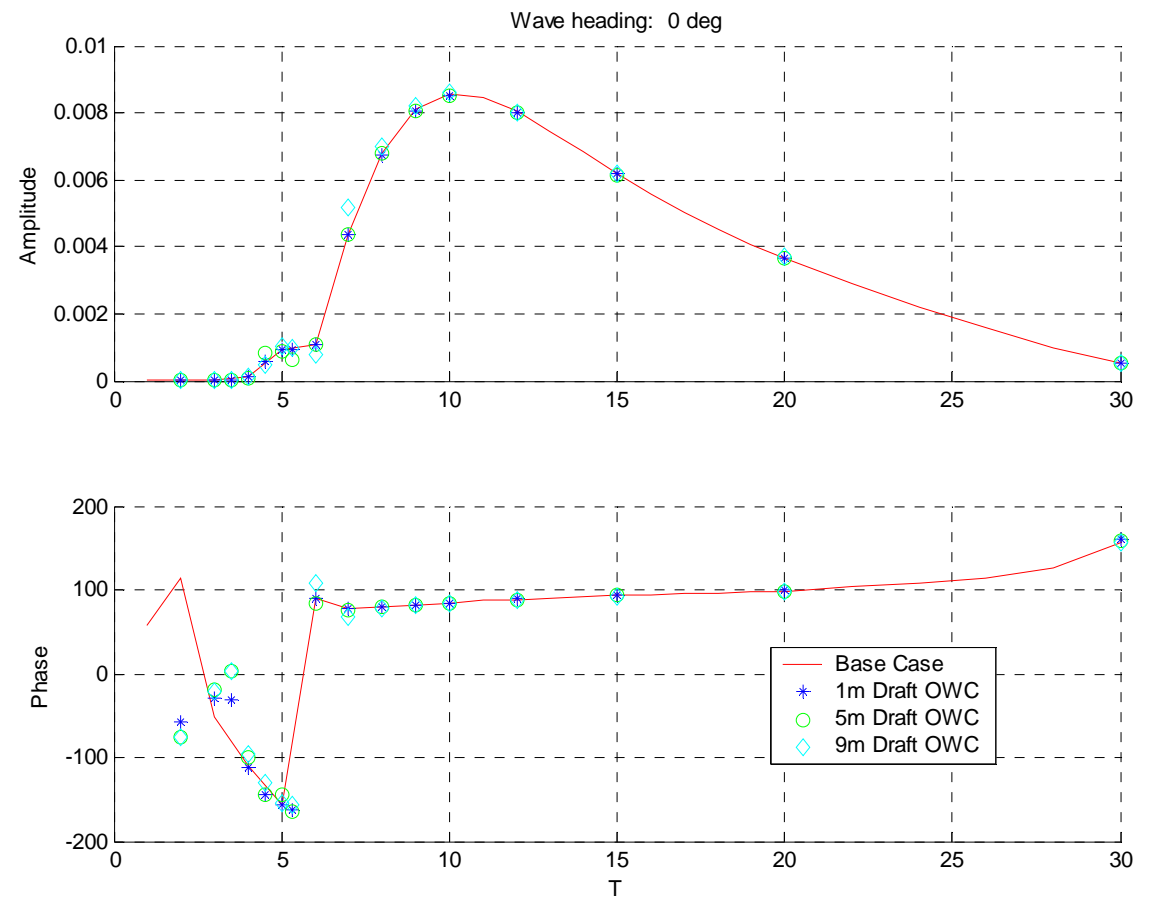

RAO in direction 5

Figure 26: Pitch RAO (amplitude in rad/m, phase in deg) of OWC WindFloat for various drafts with $\mathrm{D}_{\mathrm{Owc}}=14 \mathrm{~m}$
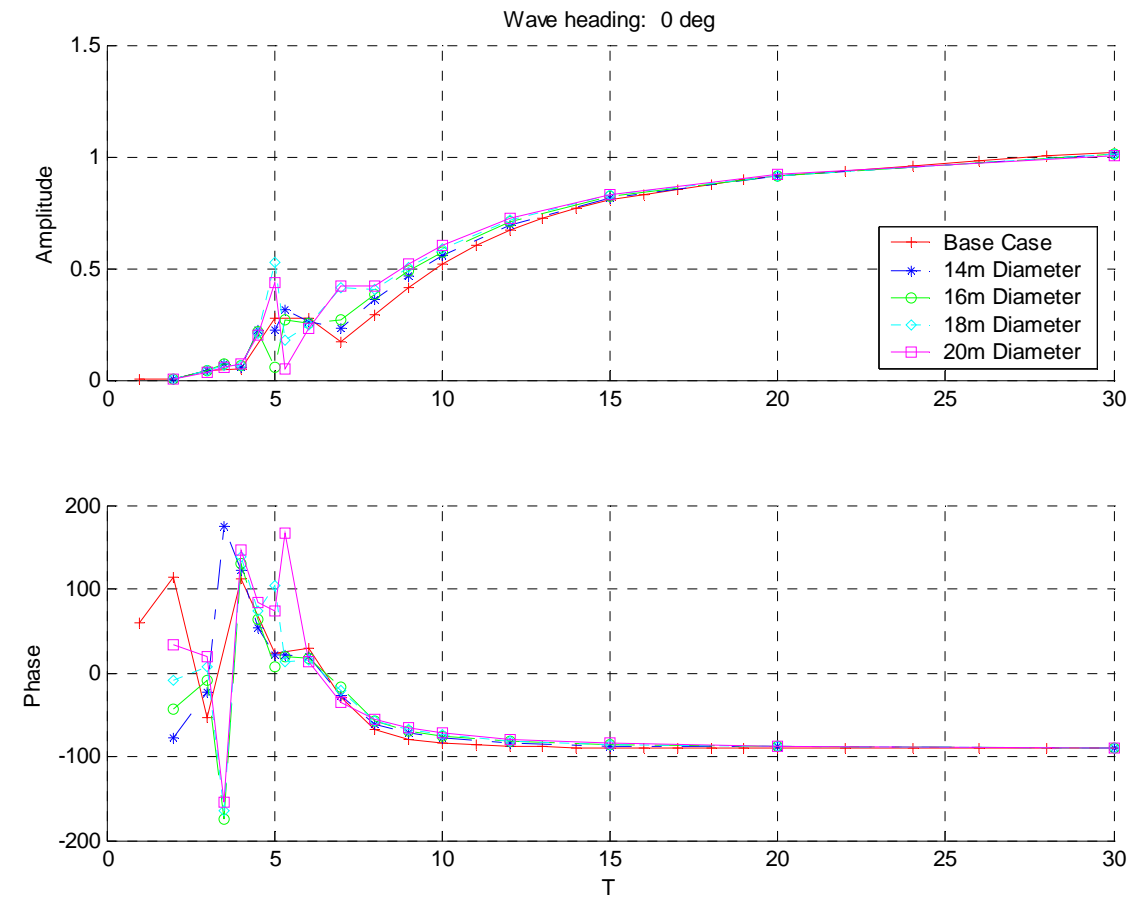

RAO in direction 1

Figure 27: Surge RAO (amplitude in $\mathrm{m} / \mathrm{m}$, phase in deg) of OWC WindFloat for various diameters with $\mathrm{T}_{\mathrm{Owc}}=9 \mathrm{~m}$ 

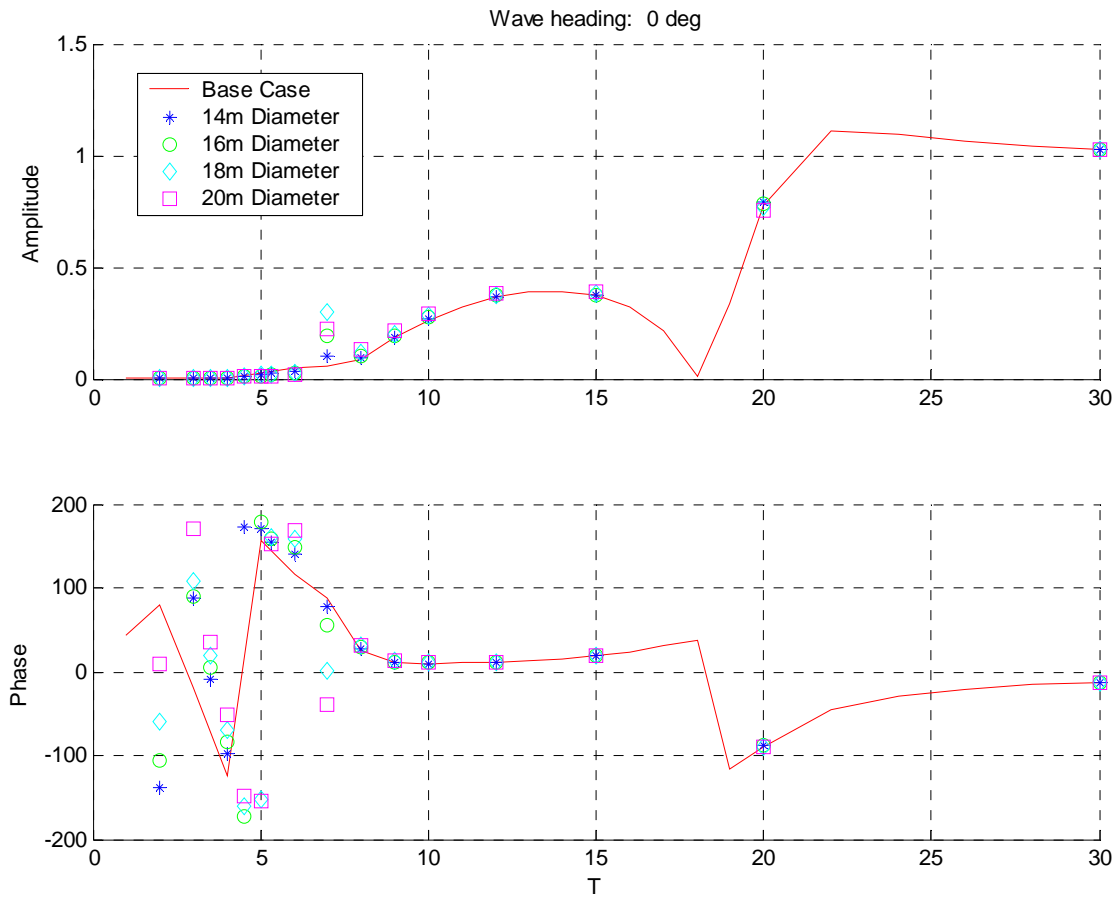

RAO in direction 3

Figure 28: Heave RAO (amplitude in $\mathrm{m} / \mathrm{m}$, phase in deg) of OWC WindFloat for various diameters with $\mathrm{T}_{\mathrm{owc}}=9 \mathrm{~m}$
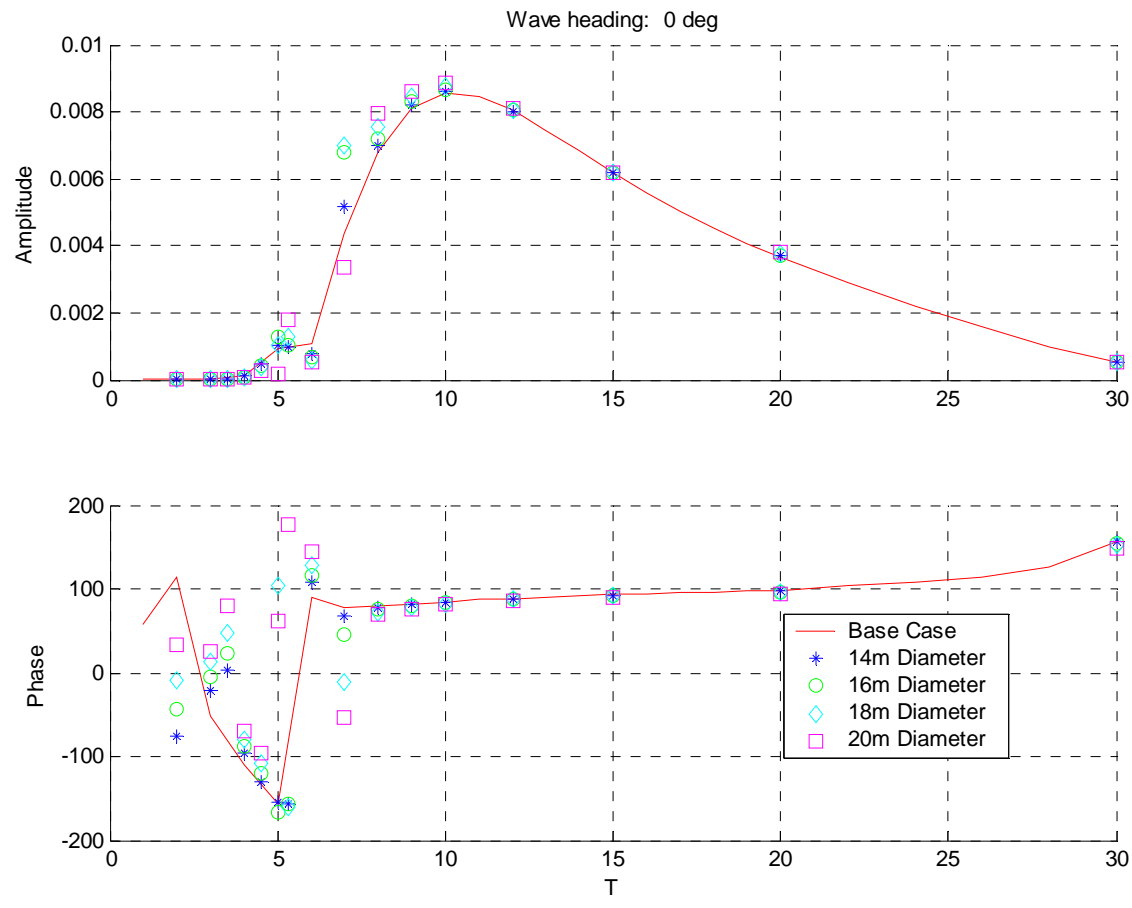

RAO in direction 5

Figure 29: Pitch RAO (amplitude in rad/m, phase in deg) of OWC WindFloat for various diameters with $\mathrm{T}_{\mathrm{Owc}}=9 \mathrm{~m}$ 


\subsubsection{Power Output}

\section{Turbine Characteristics:}

Curran et al. [9] provide a relationship between the pressure drop $\Delta \mathrm{p}_{0}$ and the axial velocity $\mathrm{U}$ :

$\Delta p_{0}=0.525 N_{p} \tan \left(\frac{\pi}{2} S\right) \times 4 \rho_{\text {air }} \Omega R U$

where $N_{p}$ is the number of planes of the turbine - usually 1 or $2-, S$ Is the solidity, $\Omega$ the rotational speed and $\mathrm{R}$ the tip radius.

Table 9 provides the turbine dimensions and characteristics that were used in the present analysis. They are adapted from the Islay turbine characteristics.

Table 9 : Turbine Characteristics

\begin{tabular}{|l|l|c|}
\hline Number of Planes & $\mathrm{N}_{\mathrm{p}}$ & 2 \\
\hline Tip Radius [m] & $\mathrm{R}$ & 0.6 \\
\hline Hub Radius [m] & $\mathrm{R}_{\mathrm{i}}$ & 0.372 \\
\hline Blade Profile & & NACA0012 \\
\hline Number of Blades & $\mathrm{N}_{\mathrm{b}}$ & 7 \\
\hline Solidity & $\mathrm{S}$ & 0.5 \\
\hline Rotational Velocity [rpm] & $\Omega$ & 1500 \\
\hline
\end{tabular}

With these characteristics, $\alpha=241.1, A_{T}=0.70 m^{2}$ and the pneumatic extracted power $P=344.3 Q_{C}{ }^{2}$.

In this analysis, we assume a maximum efficiency of 0.6 , so that $\mathrm{P}_{\text {converted }}=344.3 \eta(U / \Omega R) \mathrm{Q}_{c}{ }^{2}$.

\section{Power RAOs:}

This turbine was designed for optimal operation in combination with an OWC chamber with a free surface area around $36 \mathrm{~m}^{2}$. For larger internal free surface, the efficiency drops due to large flow ratios.

For a $14 \mathrm{~m}$ diameter OWC, a $1 / 4$ of the internal annulus represents $18.8 \mathrm{~m}^{2}$. Internal vertical walls at angle $\theta_{1}=135^{\circ}$ and $\theta_{2}=225^{\circ}$ are assumed to enclose the OWC chamber. The walls were not represented in the diffraction-radiation analysis and may affect the results slightly. This represents one quarter of the entire cylindrical chamber, and the results also assume only one column is fitted with the OWC.

RAOs of pneumatic power and converted power are plotted in Figure 30 and Figure 31 respectively for different OWC draft. The pneumatic power is the power available to the turbine due to the flow rate across its blades and to the pressure drop. The converted power is the amount of power the turbine 
actually manages to extract. The difference is due to the efficiency of the turbine, which varies significantly for different devices.

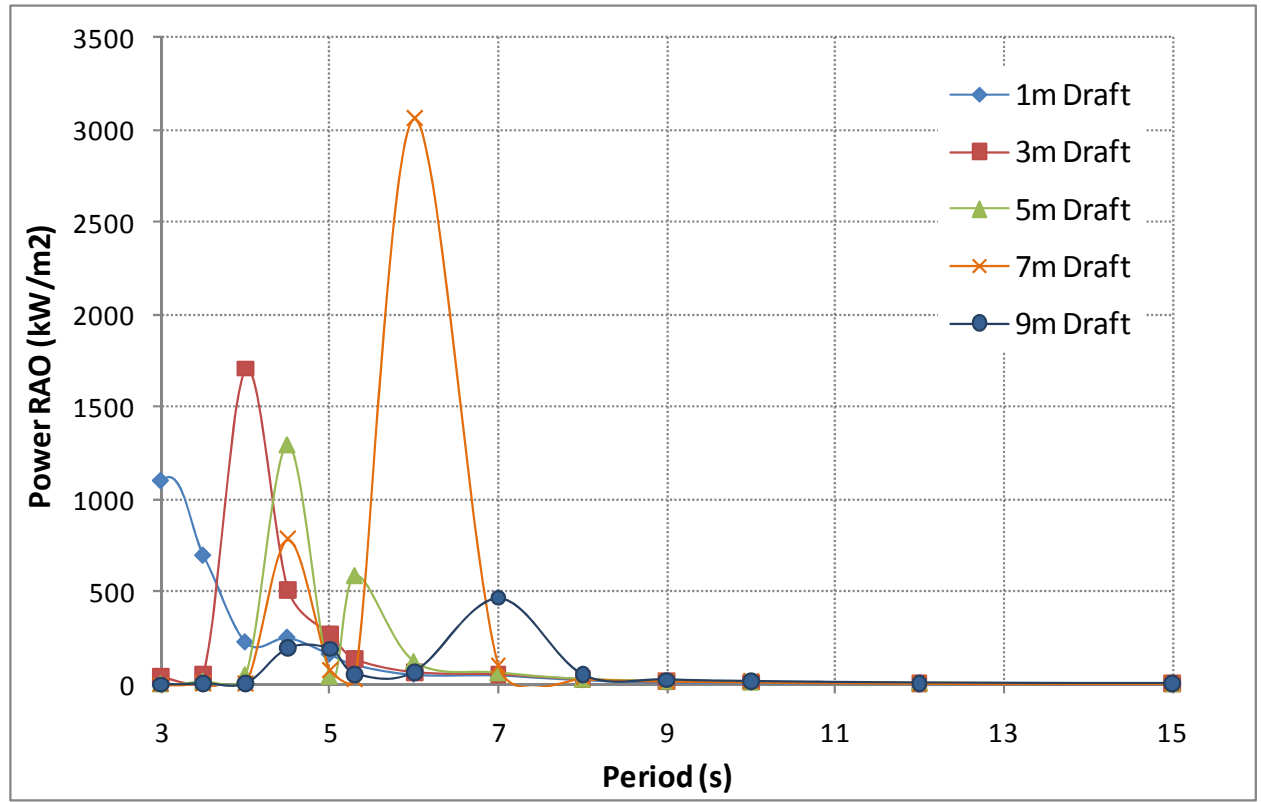

Figure 30: RAOs of Average Pneumatic Power (kW/ m2 of incident wave) - AC=18.8m2

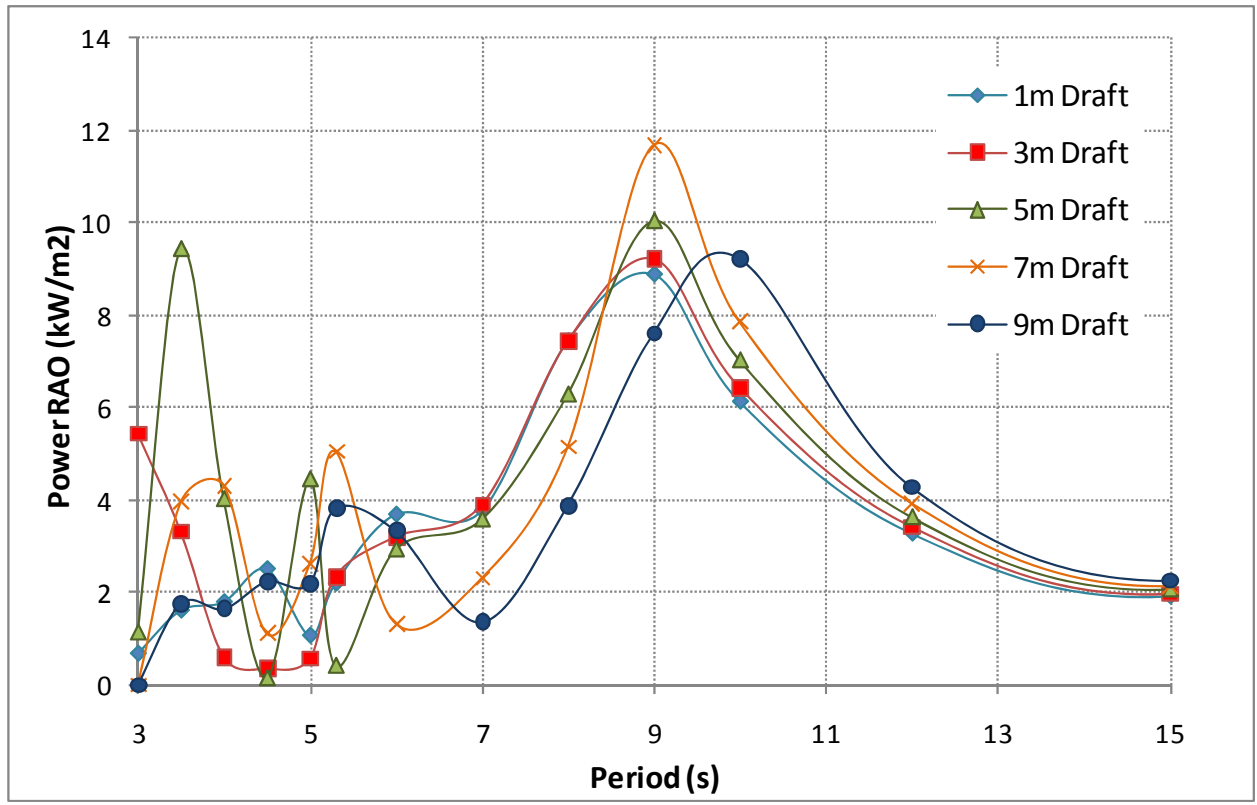

Figure 31: RAOs of Average Converted Power $\left(\mathrm{kW} / \mathrm{m}^{2}\right.$ of incident wave $)-A_{C}=18.8 m^{2}$ 
The pneumatic power curves show the effect of OWC resonance. However the resonant periods do not majorly affect the converted power curves. In the latter case, high flow ratios cancel the effect of resonance due to low turbine efficiency.

Figure 32 shows the flow ratios for period T=4, 6 and 9 seconds for the $7 \mathrm{~m}$ draft, $14 \mathrm{~m}$ diameter OWC. At the period of resonance, $\mathrm{T}=6 \mathrm{sec}$, the flow ratio exceeds 0.2 more than $90 \%$ of the time. The efficiency of the turbine is very low. Conversely, at $T=9 \mathrm{sec}$, the amplitude of the flow ratio is optimum, and the turbine operates at its maximum efficiency. The corresponding variations of efficiency are plotted in Figure 33 for each period. At $\mathrm{T}=9 \mathrm{sec}$, the efficiency is 0.6 except when the turbine stalls at very low flow rates. This explains the peaks of converted power at 8 and $9 \mathrm{sec}$, regardless of OWC geometry. It highlights the importance of tuning turbine characteristics to target specific sea-states and be compatible with the OWC geometry. This optimization can be achieved by varying turbine rotational speed or diameter for instance.

It should be noted that the converted power curves are valid for $1 \mathrm{~m}$ high waves only. Larger waves will produce greater flow ratios and lower the efficiency; the power RAOs are likely to be lower.

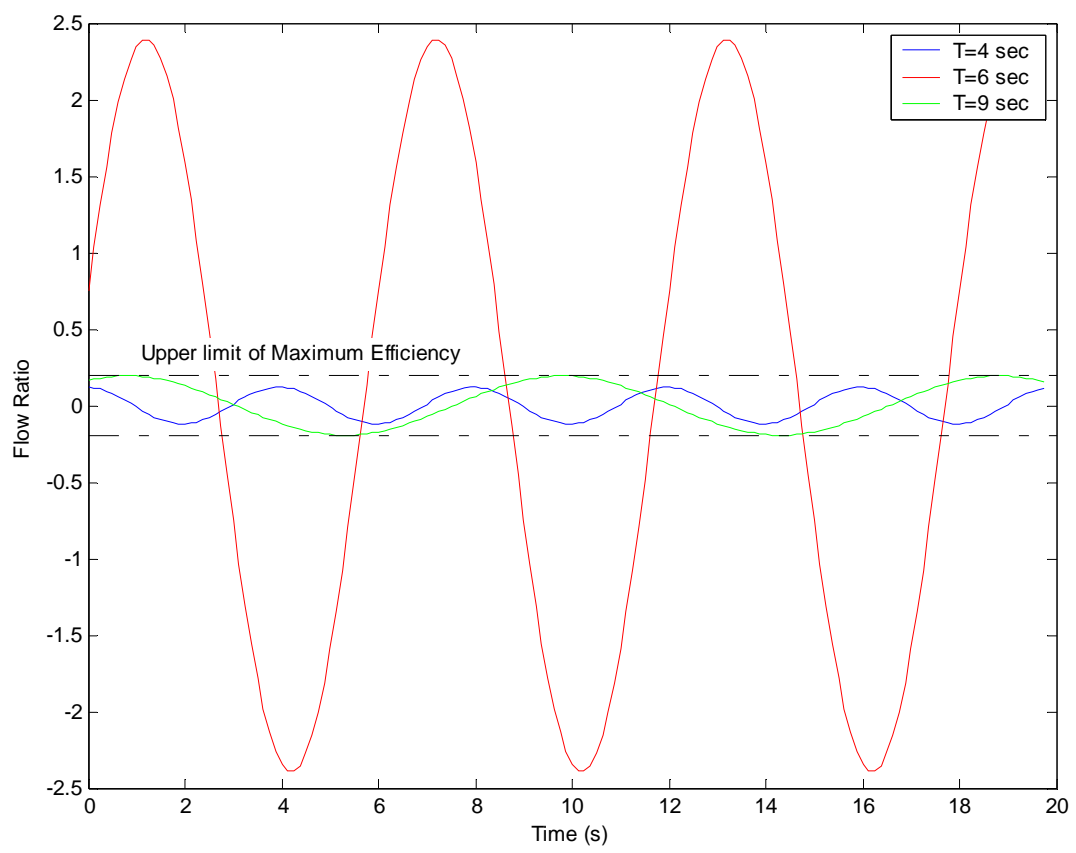

Figure 32: Variations of Flow Ratio $-\mathrm{T}_{\mathrm{owc}}=7 \mathrm{~m}, \mathrm{D}_{\mathrm{owc}}=14 \mathrm{~m}$ and $\mathrm{OWC}$ natural period $=6 \mathrm{~s}$. 

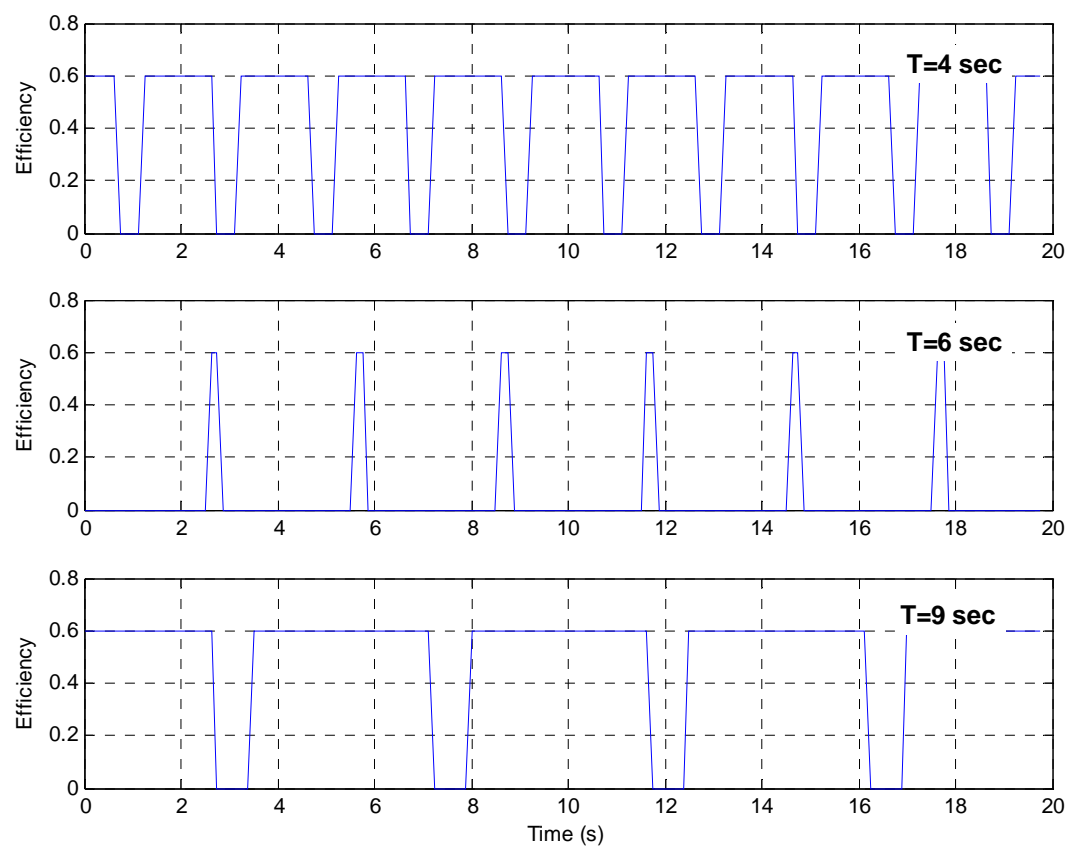

Figure 33: Variations of Efficiency $-T_{o w c}=7 m, D_{o w c}=14 m$ and $0 W C$ natural period $=6 \mathrm{~s}$.

The effect of WindFloat motions on the generation of pneumatic power was also investigated.

In section 2.2.2, the volumetric air flow $Q_{c}$ is expressed as the sum of two terms: a motion induced term and a wave elevation term. Radiation effects are also included in the wave elevation term since platform motion creates radiated waves. These two terms are plotted independently in Figure 34 to Figure 36.

At low wave periods, the WindFloat RAOs are small and the total volumetric flow rate is equal to the flow rate induced by the internal free surface, as shown for $T=6 \mathrm{~s}$. Around the heave period of resonance of the platform, at $\mathrm{T}=20 \mathrm{sec}$, the platform motions are significant and increase the total volumetric flow rate. At large periods though, the two terms are out of phase, since the platform goes up and down with the wave. The platform motion contributes to lower the forces on the air column. 


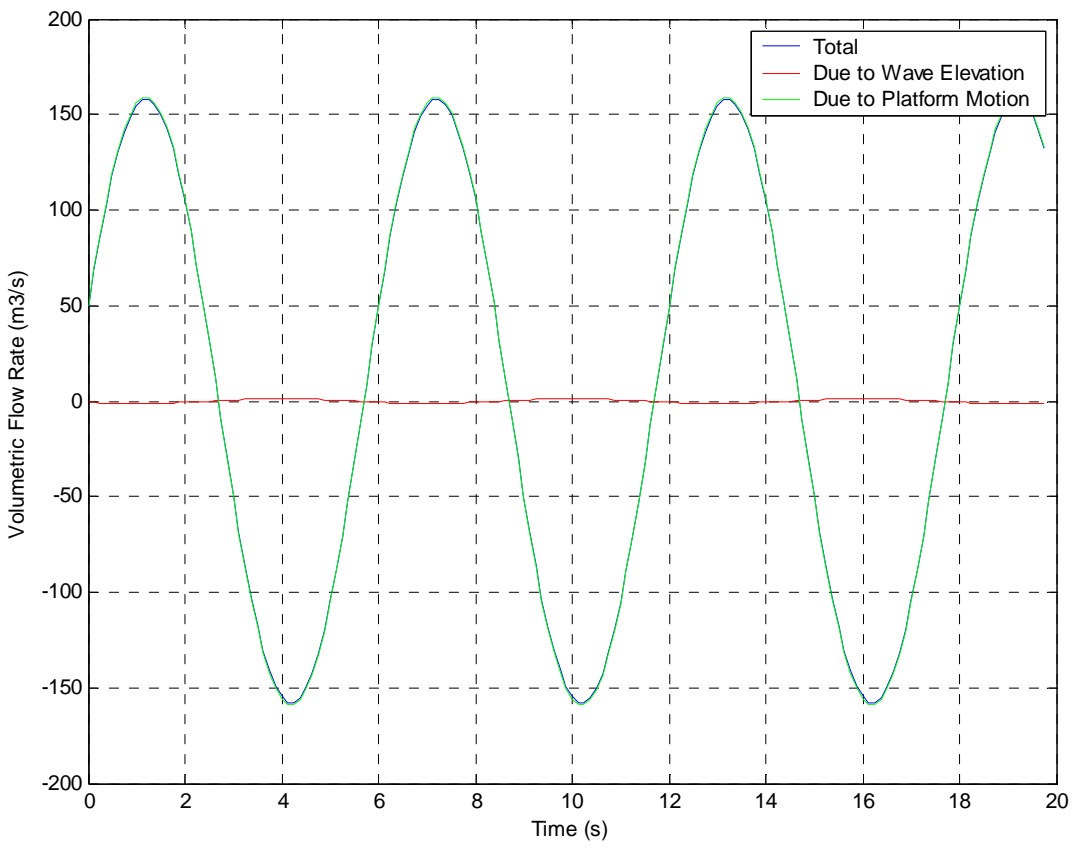

Figure 34: Effects of Relative motion and internal free surface on volumetric flow rate $\mathrm{T}_{\mathrm{owc}}=7 \mathrm{~m}, \mathrm{D}_{\mathrm{owc}}=14 \mathrm{~m}$ and wave characteristics $\mathrm{H}=1 \mathrm{~m}, \mathrm{~T}=6 \mathrm{sec}$

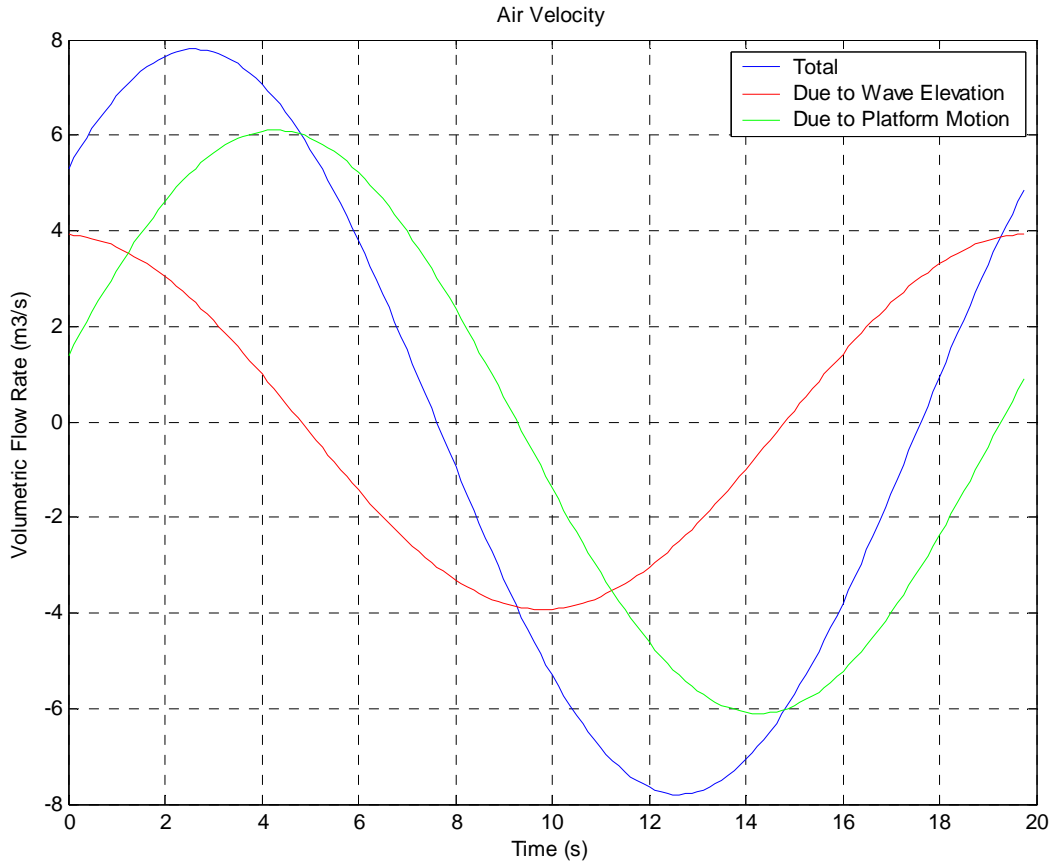

Figure 35: Effects of Relative motion and internal free surface on volumetric flow rate TOWC $=7 \mathrm{~m}, \mathrm{DOWC}=14 \mathrm{~m}$ and wave characteristics $\mathrm{H}=1 \mathrm{~m}, \mathrm{~T}=20 \mathrm{sec}$ 


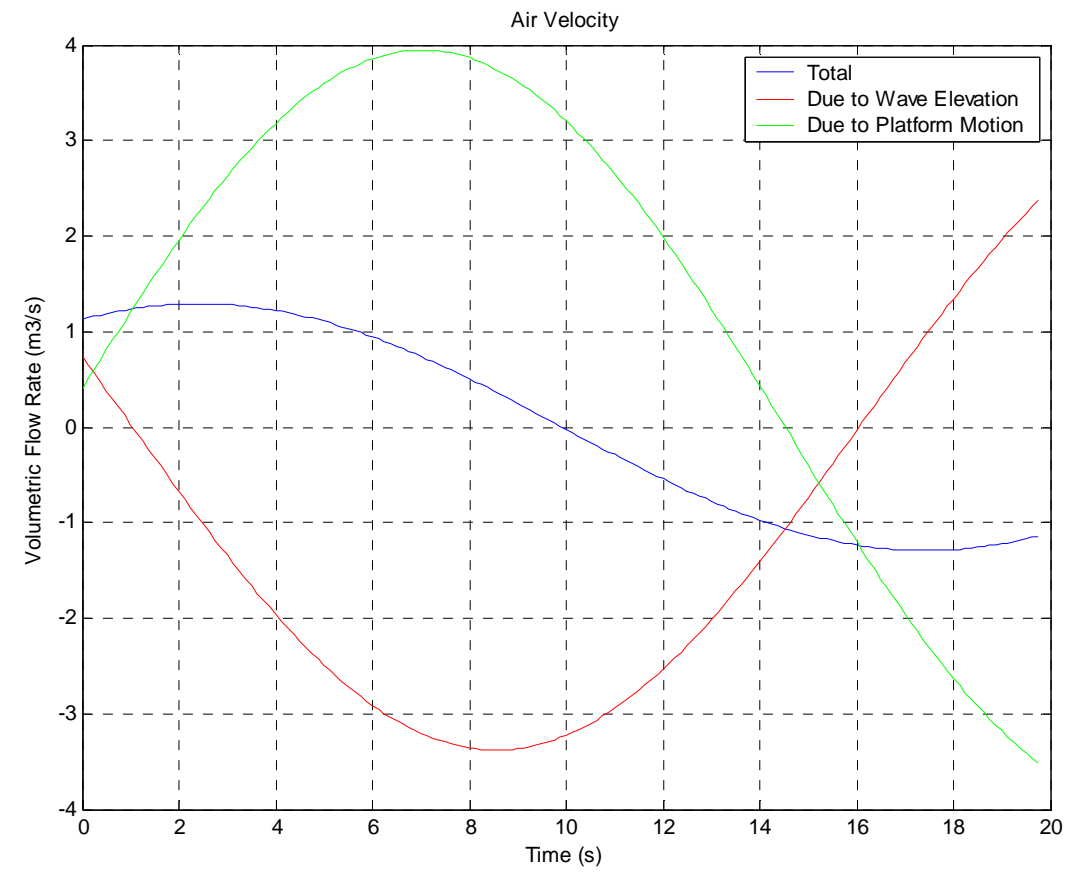

Figure 36: Effects of Relative motion and internal free surface on volumetric flow rate TOWC $=7 \mathrm{~m}, \mathrm{DOWC}=14 \mathrm{~m}$ and wave characteristics $\mathrm{H}=1 \mathrm{~m}, \mathrm{~T}=30 \mathrm{sec}$

\subsection{Point Energy Absorber}

\subsubsection{Single Device (SWEDE)}

\section{Resonance frequency}

The resonance frequency $\sigma_{\text {res }}$ in heave of the SWEDE is computed by:

$$
\sigma_{r e s}=\sqrt{\frac{k}{m+\mu}}
$$

Where $k=\rho g A_{w p}$ is the total spring constant of the system, $\mathrm{m}$ the mass of the floater, and $\mu$ the added-mass of the floater.

Table 10 : SWEDE Characteristics

\begin{tabular}{|l|r|}
\hline Sphere spring constant $\mathbf{k}$ & $770 \mathrm{kN} / \mathrm{m}$ \\
\hline Sphere mass/displacement $\mathbf{m}$ & $261.80 \quad$ tonnes \\
\hline Sphere heave added-mass $\boldsymbol{\mu}$ & $125.69 \mathrm{tonnes}$ \\
\hline Frequency of resonance $\boldsymbol{\sigma}_{\text {res }}$ & $1.41 \mathrm{rad} / \mathrm{s}$ \\
\hline Period of resonance $\boldsymbol{T}_{\text {res }}$ & $4.46 \mathrm{~s}$ \\
\hline
\end{tabular}


A numerical decay test is performed and confirms that the resonance period is $4.5 \mathrm{~s}$ in heave.

\section{Base Case}

For the SWEDE, the base case consists of the following parameters:

- Regular sinusoidal wave of $3 m$,

- $\quad$ Line damping of $100 \mathrm{kN} . \mathrm{s} / \mathrm{m}$.

The six degree-of-freedom RAOs of the spherical floater are presented on Figure 37 . The heave resonance period at $4.5 \mathrm{~s}$ appears clearly on the graph.

The floater picks up a surge motion that is transformed into a significant pitch motion, because the three lines apply varying tensions on the floater during the motion.

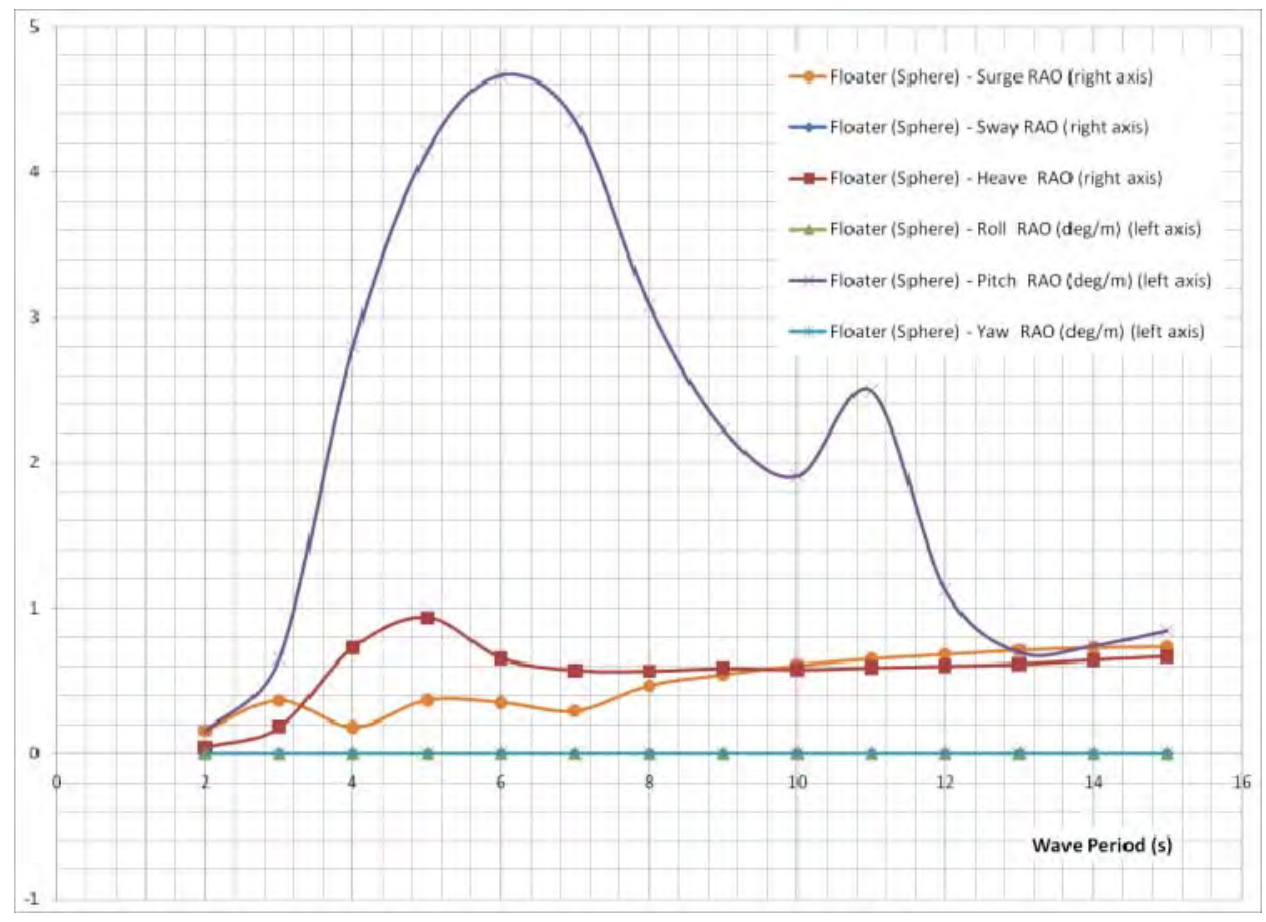

Figure 37: SWEDE RAOs

\section{Comparison WindFloat and WWF}

The SWEDE WWF base case is compared to the WindFloat base case on Figure 38. The major difference appears in the surge RAO. The WindFloat surge RAO decreases when the spherical floater is attached to the platform, because the floater harnesses part of this typical WindFloat pitch motion. 

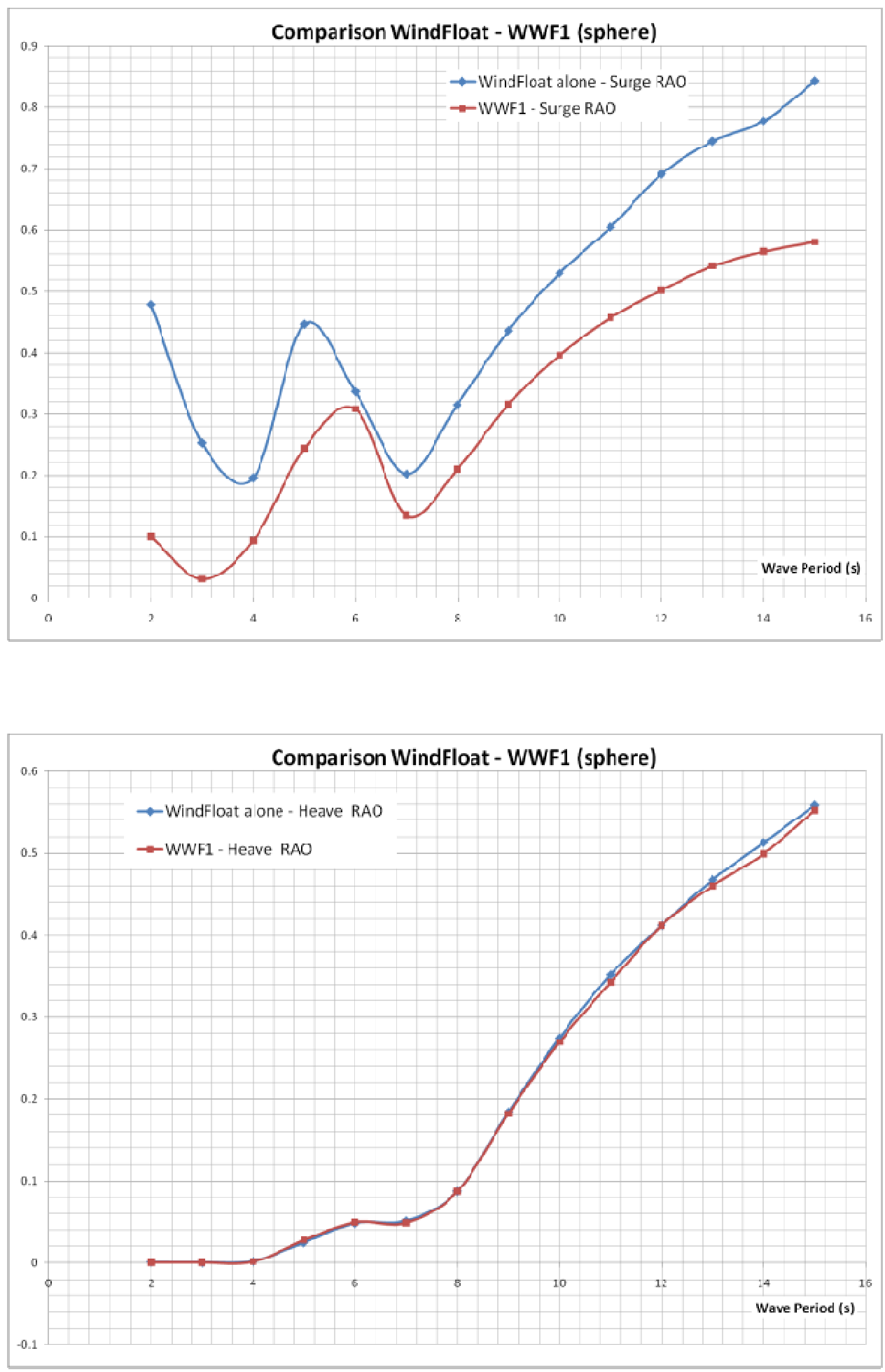


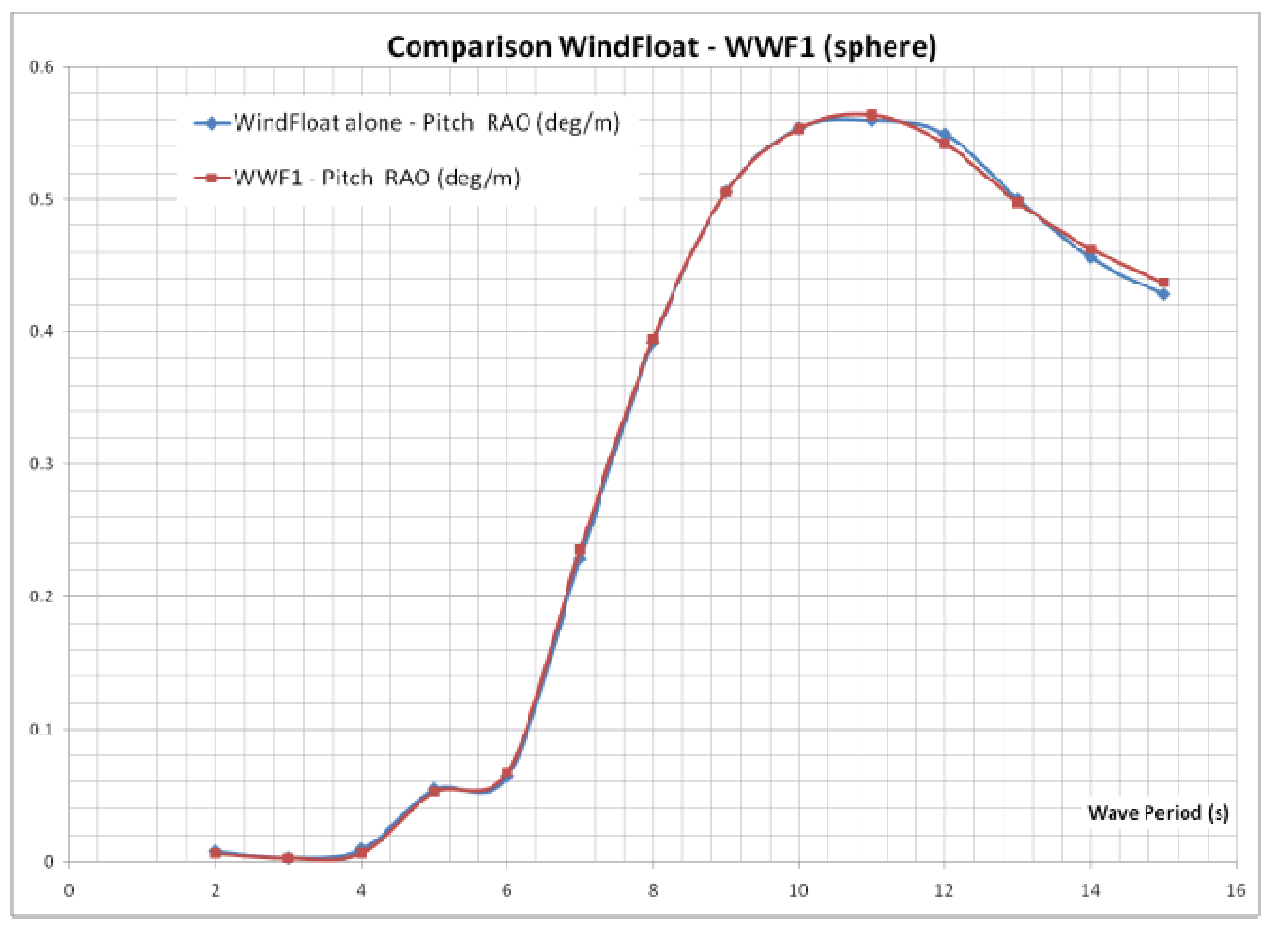

Figure 38a, b, and c: Comparison of the WindFloat RAOs vs. the WWF-SWEDE RAOs for Surge (a), Heave (b) and Pitch (c)

\section{Power curves}

The average mechanical energy $P_{\text {me }}$ received by one line is computed using the following equation:

Where $F(t)$ is the line tension at the time $t, F_{0}$ is the line pretension, and $v(t)$ is the line velocity at the time t.

The results of the numerical simulations are presented on Figure 39. The power per wave amplitude squared is given for the three lines holding the sphere, for different damping levels (one color per damping) with respect to the wave period.

The conclusions are the following:

- $\quad$ The maximum power of about $50 \mathrm{~kW} / \mathrm{m}^{2}$ is obtained for a damping of $200 \mathrm{kN} . \mathrm{s} / \mathrm{m}$ at a $3 \mathrm{~s}$ wave period, and for a damping of $1000 \mathrm{kN} . \mathrm{s} / \mathrm{m}$ at a $5 \mathrm{~s}$ wave period.

- $\quad$ The surge period of resonance at $3 s$ impacts the average power significantly for smaller wave amplitudes.

- $\quad$ The higher the wave amplitude is, the more the heave resonance period of 4-5s comes into play. This can also be due to the following effect: the stiffness of the lines decreases when the pitch motion amplitude increases (for higher wave amplitudes), and thus the resonance period increases. 

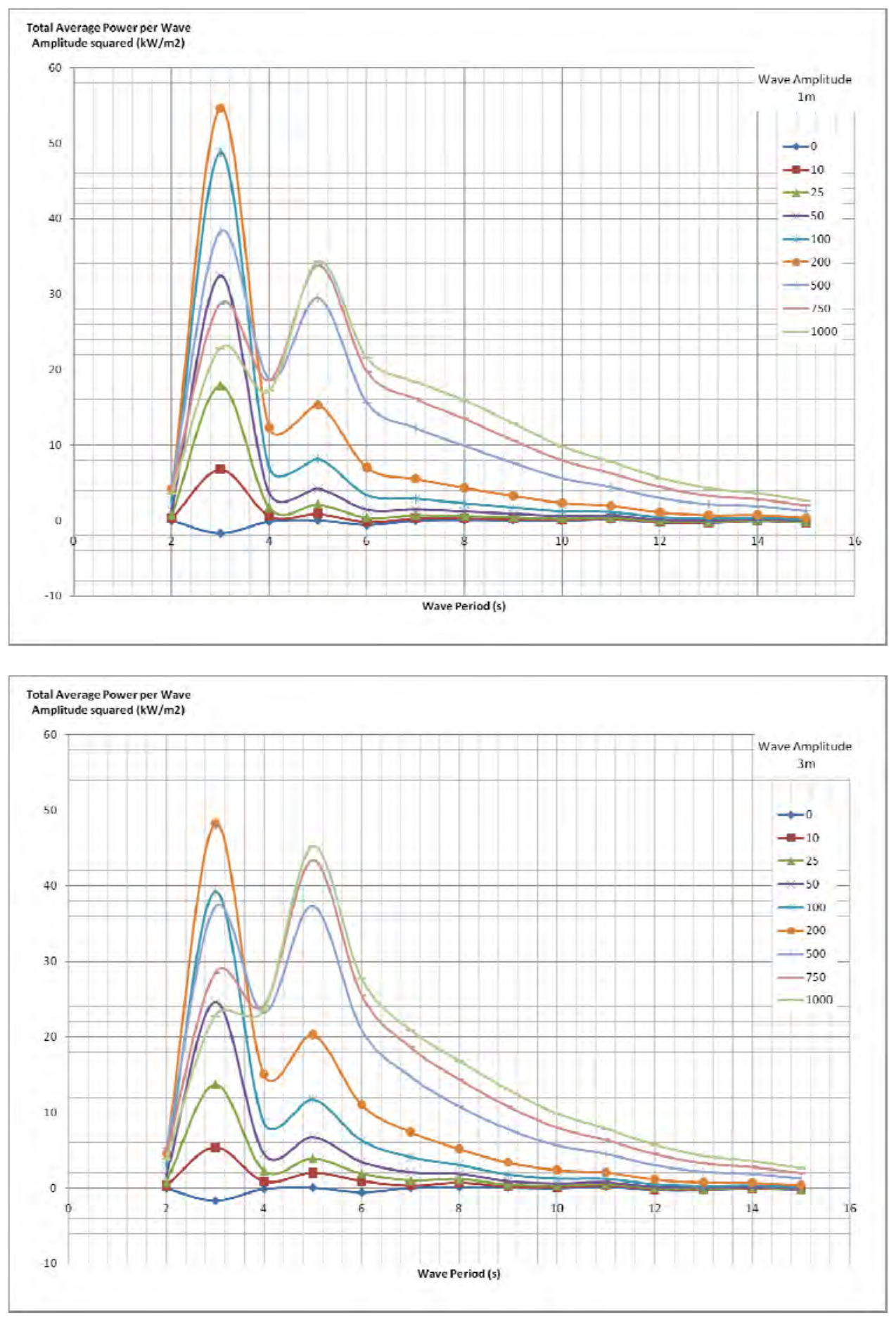

Wind Wave Float Development

DOE GRANT DE-EEOOO2652

Page 41 


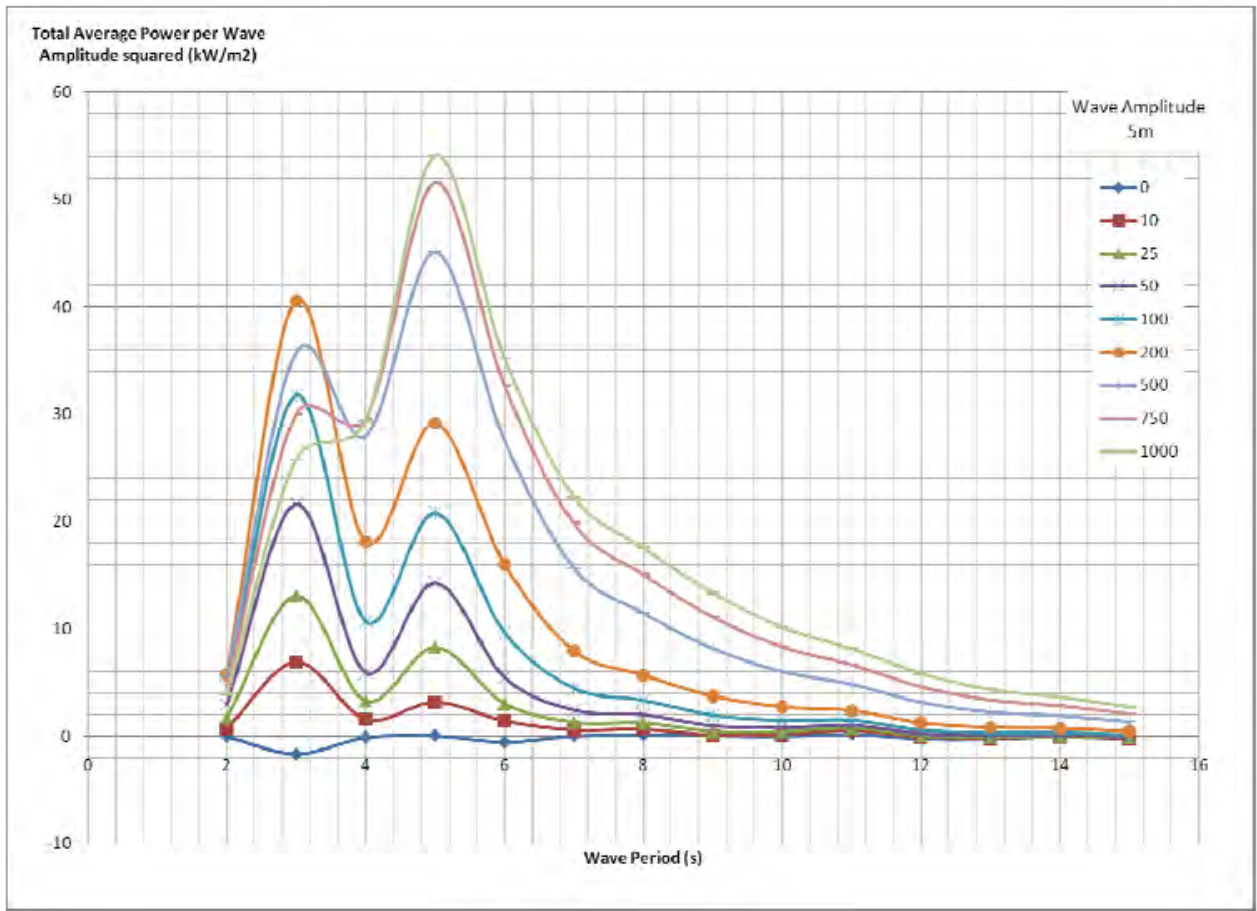

Figure 39 a, b, c: Total Average Power per Wave Amplitude squared vs. Wave Period, for wave amplitudes of $1 \mathrm{~m}-3 \mathrm{~m}$ and $5 \mathrm{~m}$, and for 9 different line dampings.

\subsubsection{Multiple Devices (Vertically constrained)}

\section{Resonance frequency}

In the case of the cylindrical floaters, the lines in a parallel configuration will have an impact on the heave resonance frequency of the device. They can be modeled as an equivalent spring constant $k_{\text {mooring }}$. The resonance frequency in heave of one cylindrical floater is computed by:

Where is the total spring constant of the system, $m$ the mass of the floater, and the added-mass of the floater. 
Table 11 : Multiple Verically constrained point absorber Characteristics

\begin{tabular}{|l|rl|}
\hline Cylinder spring constant $\mathbf{k}$ & $277.23 \mathrm{kN} / \mathrm{m}$ \\
\hline Mooring spring constant $\mathbf{k}_{\text {mooring }}$ & $500+500=1,000 \mathrm{kN} / \mathrm{m}$ \\
\hline Cylinder mass/displacement $\mathbf{m}$ & $84.82 \quad$ tonnes \\
\hline Cylinder heave added-mass & $49.10 \mathrm{tonnes}$ \\
\hline Frequency of resonance & $3.09 \mathrm{rad} / \mathrm{s}$ \\
\hline Period of resonance & $2.03 \mathrm{~s}$ \\
\hline
\end{tabular}

A decay test is performed and confirms that the resonance period is $2.05 \mathrm{~s}$ in heave.

\section{Base Case}

The same base case as the SWEDE is chosen for the cylinders. It consists of the following parameters:

- $\quad$ Regular sinusoidal wave of $3 \mathrm{~m}$,

- $\quad$ Line damping of $100 \mathrm{kN} . \mathrm{s} / \mathrm{m}$.

The heave RAOs of the three cylindrical floaters are presented on Figure 40.

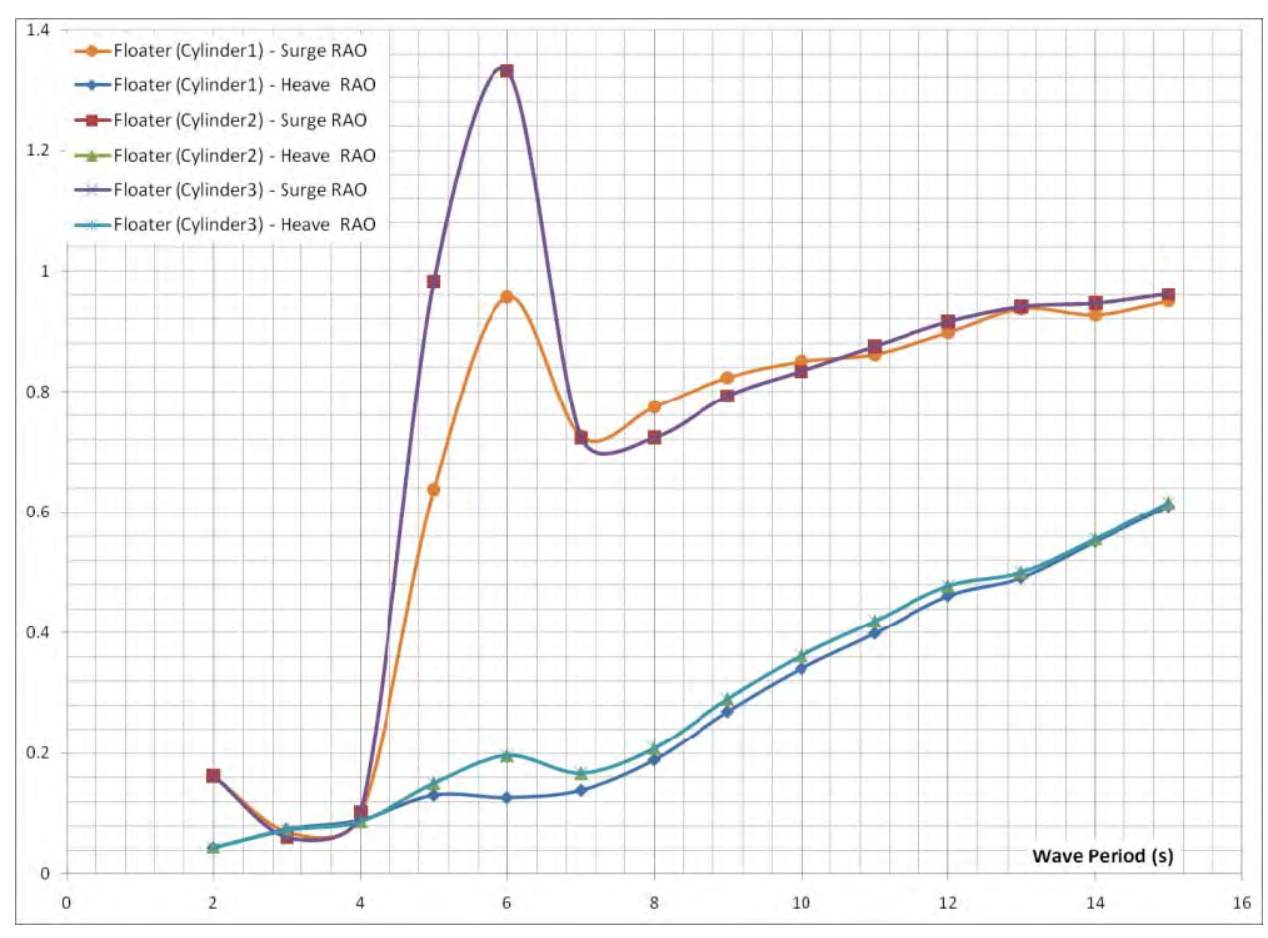

Figure 40: Heave and surge RAO of the three cylinders vs. Wave Period

Cylinder1 is the cylinder next to column 1 (the column that supports the wind turbine). The heave period of resonance at 2.05 s does not appear clearly here, because the damping level in the vertical direction $(100 \mathrm{kN} . \mathrm{s} / \mathrm{m})$ is already very high for each floater. 


\section{Comparison WindFloat and WWF}

The three-cylindrical-floater WWF base case is compared to the WindFloat base case on Figure 41.

Again, the main influence of the floaters turns out to be on the surge motion of the platform, which is reduced when the cylinders are mounted under the deck, since part of this motion is harnessed by the floaters.

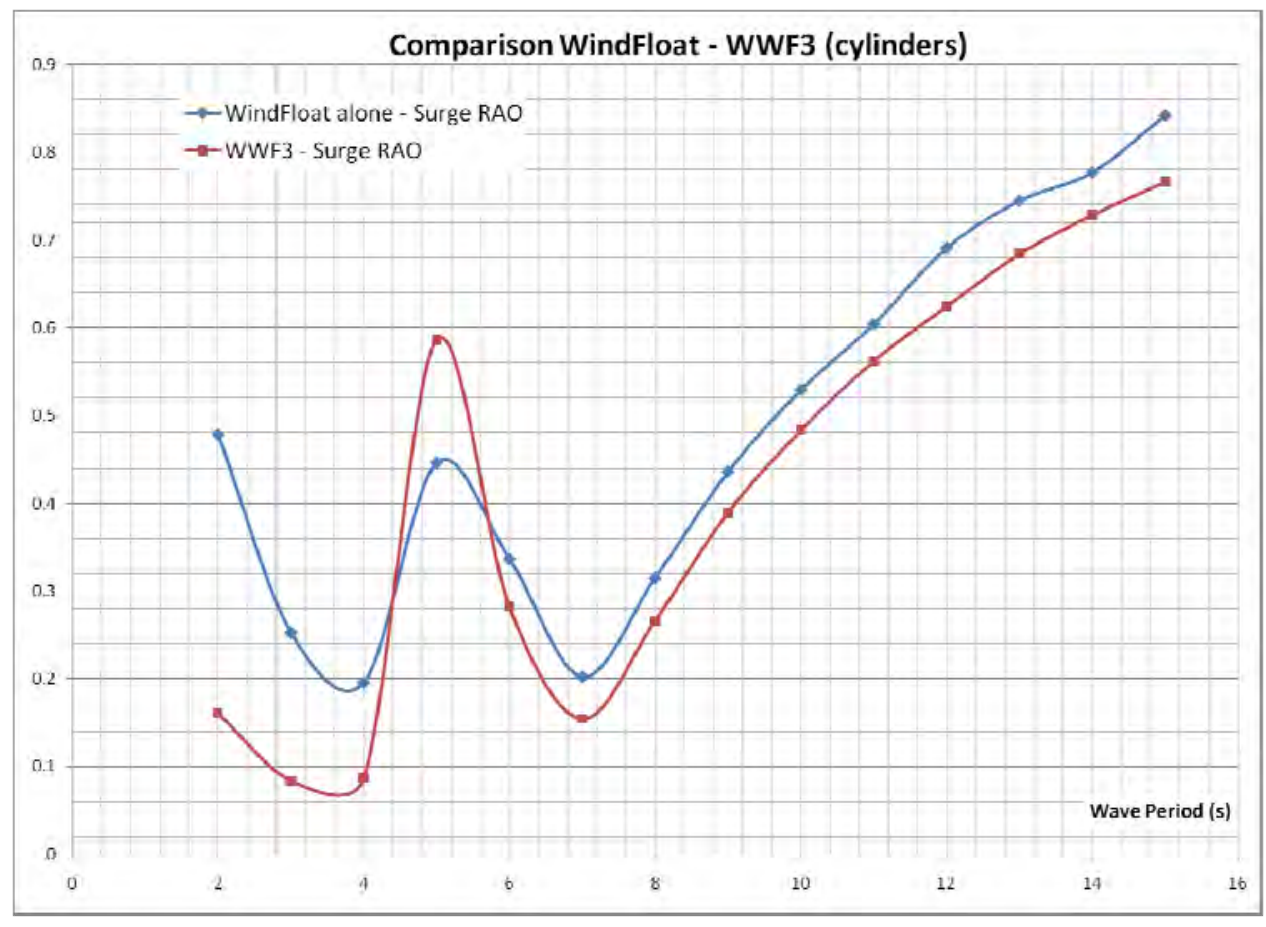



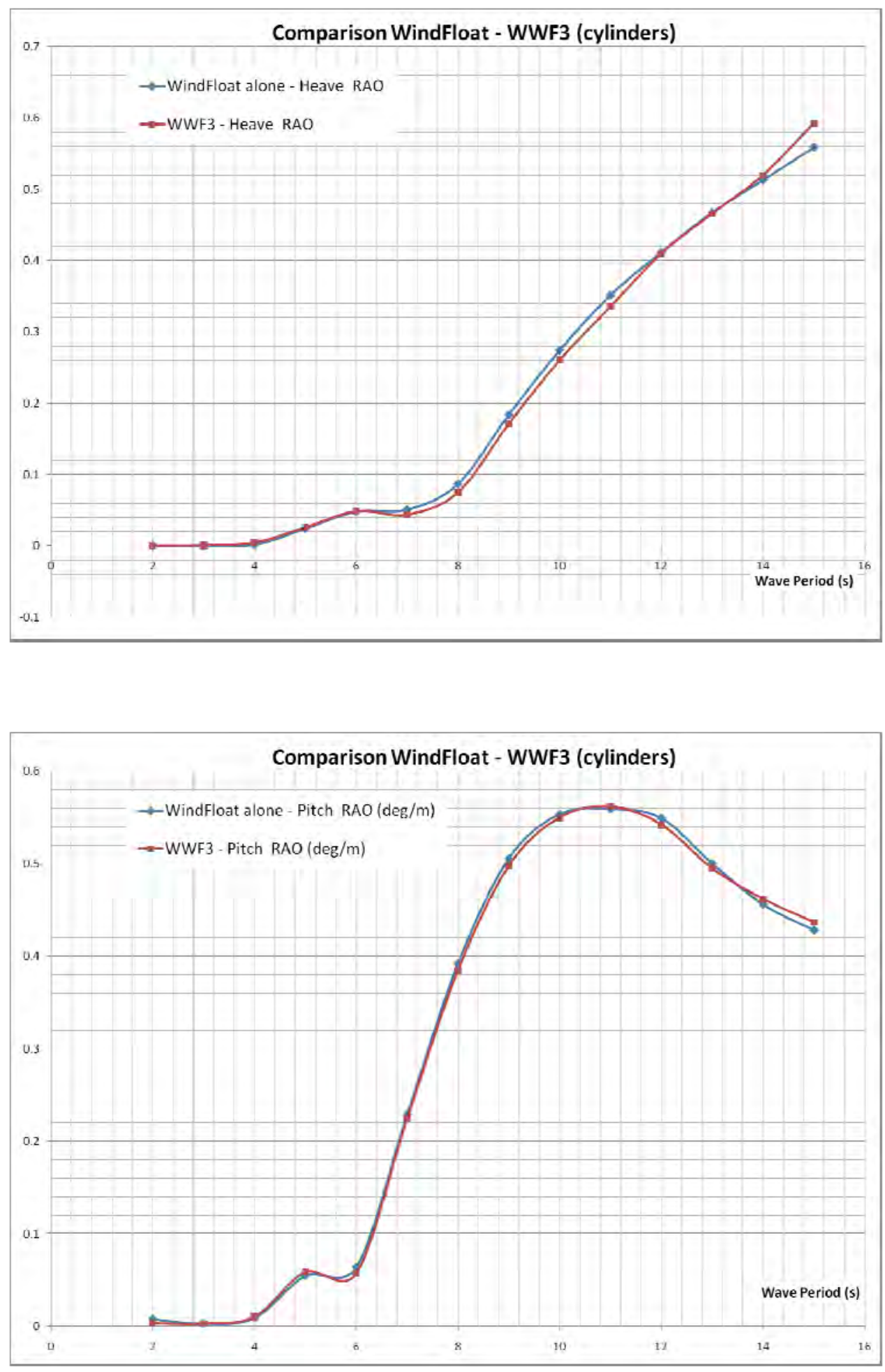

Figure 41a, b, and e: Comparison of the WindFloat RAOs vs. the 3-cylinders WWF RAOs for Surge (a), Heave(b) and Pitch(c). 


\section{Power curves}

The results of the numerical simulations are presented on Figure 42. The total power per wave amplitude squared is given for the six lines holding the cylinders, for different damping levels (one color per damping) with respect to the wave period.

The conclusions are the following:

- $\quad$ The maximum power of about $50 \mathrm{~kW} / \mathrm{m}^{2}$ is always obtained at a wave period of $5-6 \mathrm{~s}$, which corresponds to the surge resonance period.

- $\quad$ For each wave amplitude, a small power peak occurs at a $2 \mathrm{~s}$ wave period, which corresponds to the heave resonance period.

- $\quad$ The downwave cylinder (cylinder1) has power performances usually lower than cylinders 2 and 3 , the upwave cylinders.

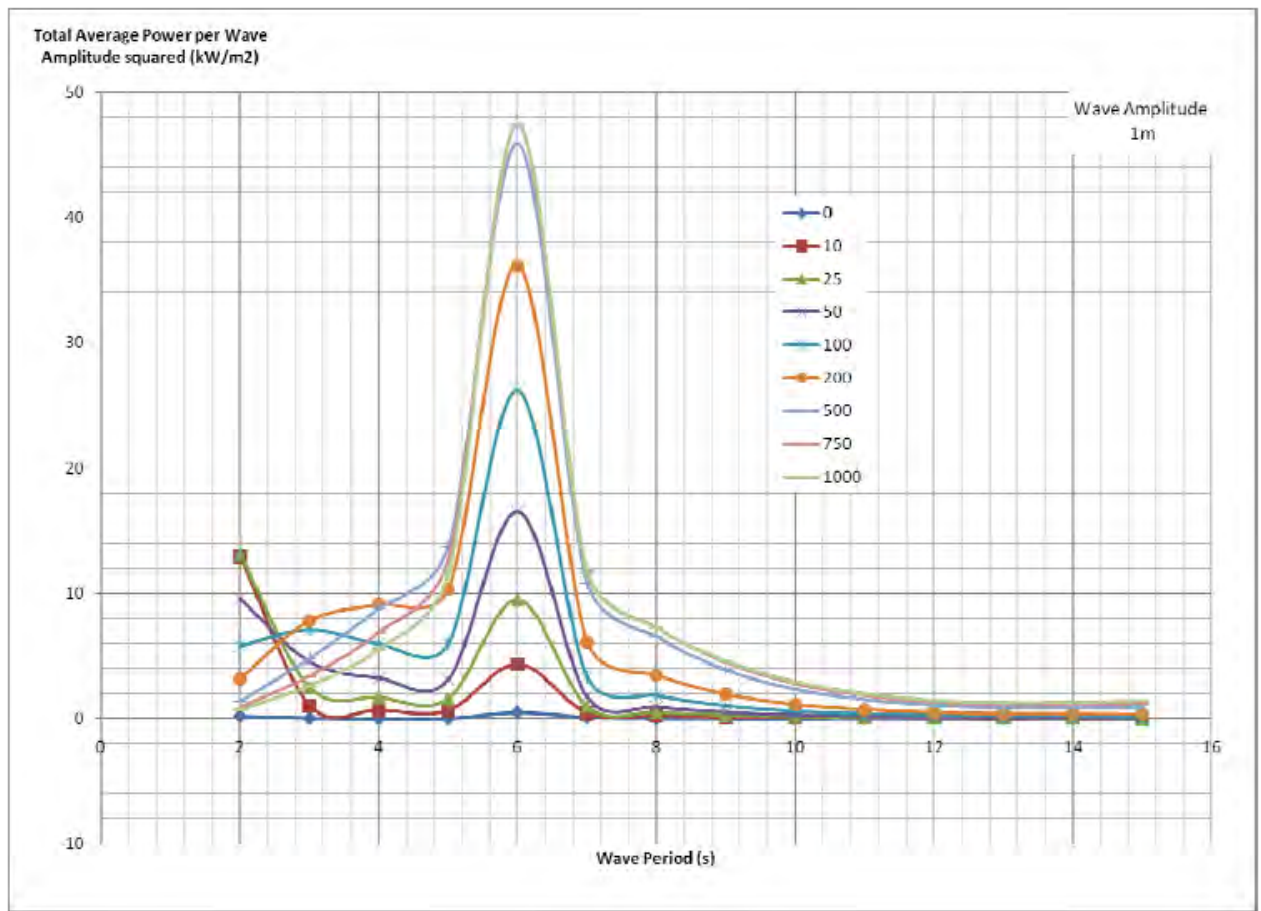



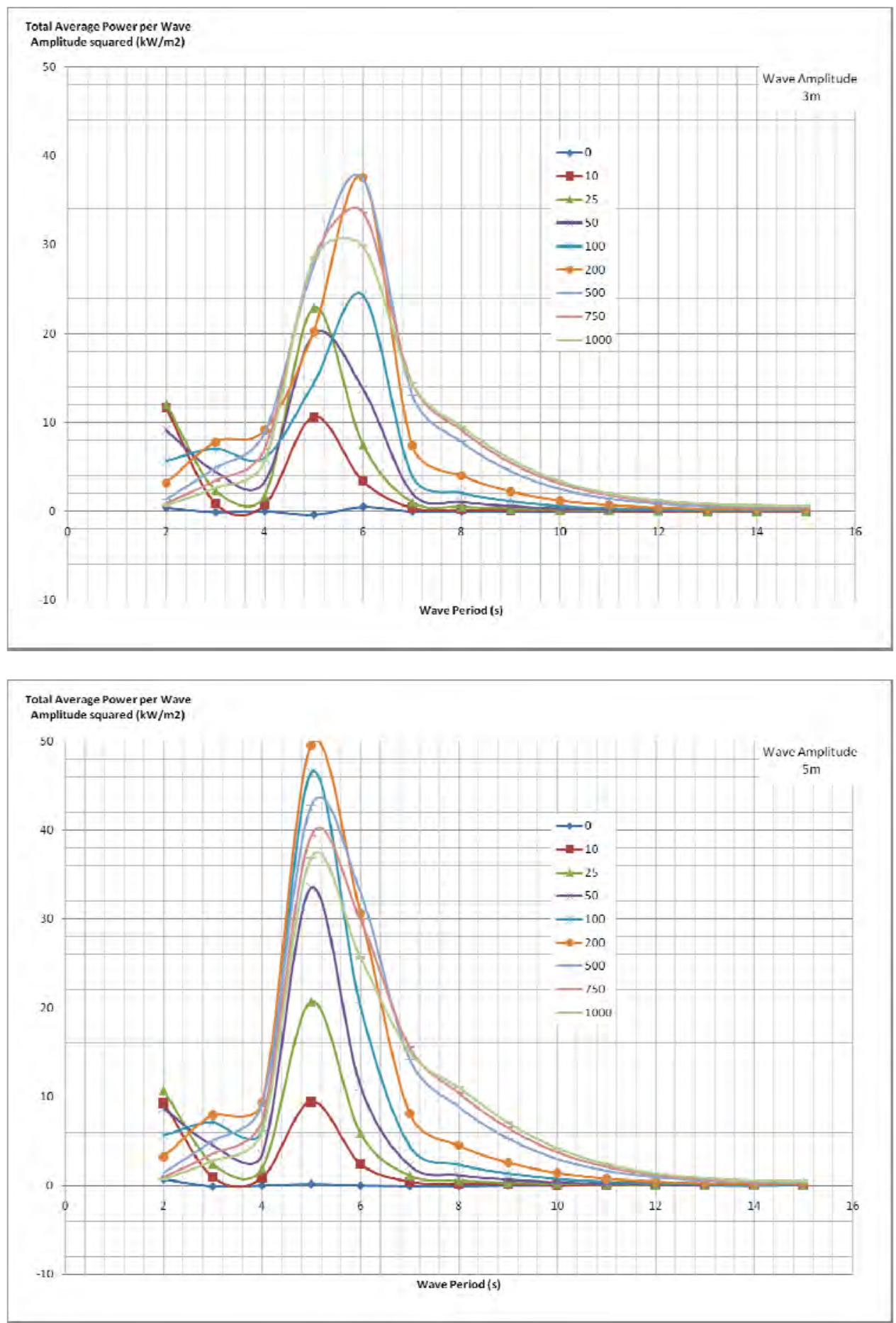

Figure 42 a, b, c: Total Average Power per Wave Amplitude squared vs. Wave Period, for wave amplitudes of $1 \mathrm{~m}-3 \mathrm{~m}$ and $5 \mathrm{~m}$, and for 9 different line dampings. 


\section{4. $\quad$ Oscillating Plates}

\section{Resonance frequency}

The waves, by hitting the flaps, induce a swinging pitch motion. The lines (spring and damper) in a parallel configuration will have an impact on the pitch resonance frequency of the device. They can be modeled as an equivalent linear spring constant $k_{\text {mooring }}$ that can be transformed into a rotational stiffness $\mathrm{k}_{\text {rot }}$. The resonance frequency $\sigma_{\text {res }}$ in pitch of one flap is computed by:

$$
\sigma_{r e s}=\sqrt{\frac{k_{r o t}}{I_{y}+\mu_{I}}}
$$

Where $k_{\text {rot }}$ is the total rotational stiffness due to the lines, applied on one flap, $\mathrm{I}_{\mathrm{y}}$ the moment of inertia, and $\mu_{I}$ the added moment of inertia, for the pitch motion.

Table 12 : Oscillating plates Characteristics

\begin{tabular}{|l|rl|}
\hline Mooring spring constant $\boldsymbol{k}_{\text {mooring }}$ & $250+250=500 \mathrm{kN} / \mathrm{m}$ \\
\hline $\begin{array}{l}\text { Mooring equivalent rotational spring } \\
\text { constant } \boldsymbol{k}_{\text {rot }}\end{array}$ & $128,000 \quad \mathrm{kN} \cdot \mathrm{m} / \mathrm{rad}$ \\
\hline Flap moment of inertia $\boldsymbol{I}_{\mathrm{y}}$ & $1715.1 \quad$ tonnes.m \\
\hline Flap added moment of inertia $\boldsymbol{\mu}_{\boldsymbol{I}}$ & $98,063 \mathrm{tonnes} \cdot \mathrm{m}^{2}$ \\
\hline Frequency of resonance $\boldsymbol{\sigma}_{\text {res }}$ & $1.10 \mathrm{rad} / \mathrm{s}$ \\
\hline Period of resonance $\boldsymbol{T}_{\text {res }}$ & $5.5 \mathrm{~s}$ \\
\hline
\end{tabular}

A decay test is performed and confirms that the resonance period is around $6 \mathrm{~s}$ in pitch.

\section{Base Case}

For the flaps, the base case consists of the following parameters:

- Regular sinusoidal wave of $3 \mathrm{~m}$,

- $\quad$ Line damping of $100 \mathrm{kN} . \mathrm{s} / \mathrm{m}$.

The pitch RAO of the three flaps is presented on Figure 43. The pitch resonance period at 6-7s appears clearly on the graph for the three flaps. Flap 1 is the flap directly perpendicular to the incoming waves. For all wave periods, its pitch RAOs appear to be higher than for the other two flaps (2 and 3), positioned at a 60-degree angle with the incoming waves. This higher pitch RAO of flap 1 has a direct impact on its resonance period in pitch, since the rotational stiffness of the lines decreases when the pitch motion amplitude increases. That is why flap 1 has a resonance period higher than $6 \mathrm{~s}$ and closer to 7s. The pitch RAOs of flap 2 and 3 are identical since they are symmetric with respect to the plan $0 x z$. 


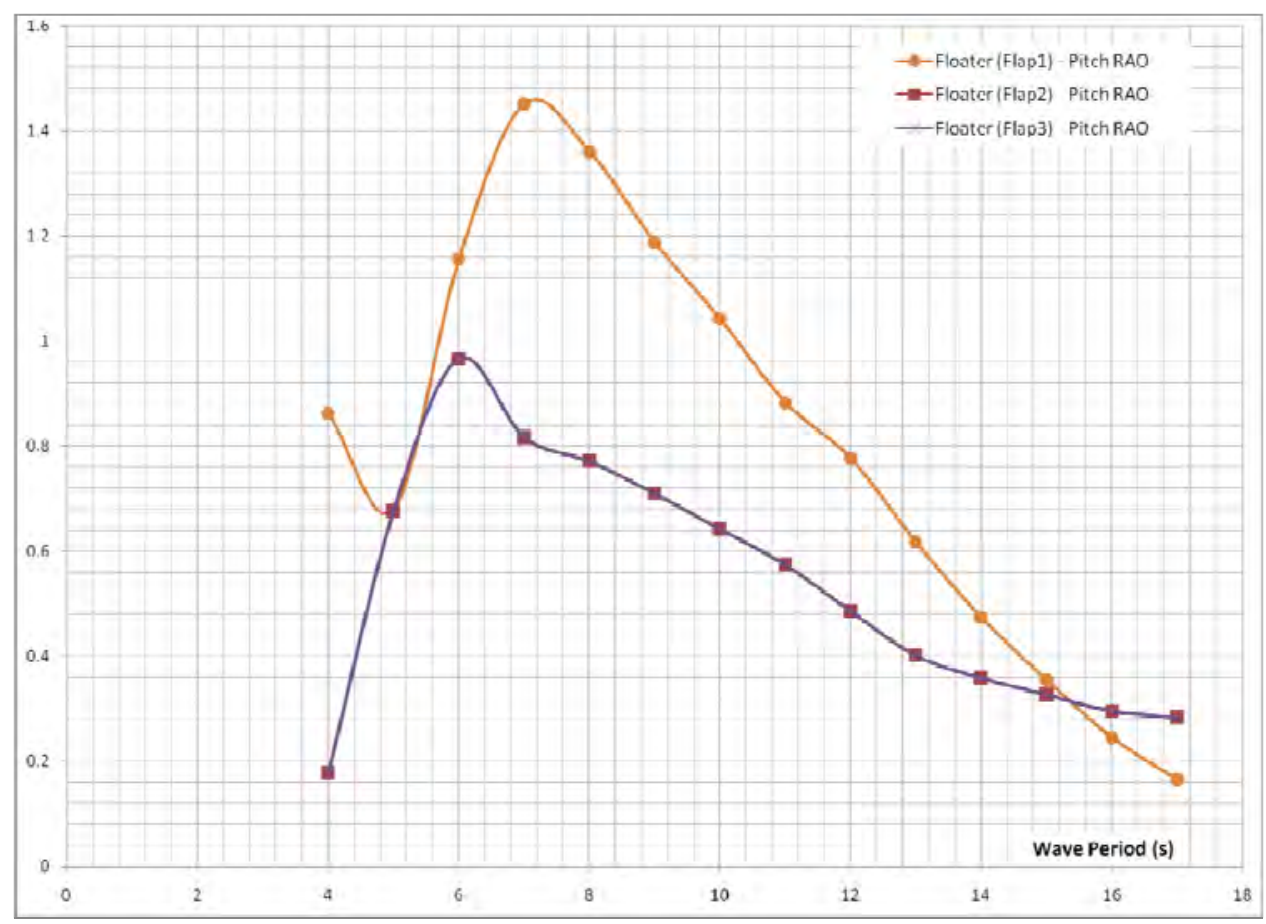

Figure 43 : Pitch RAO (deg/m) of the three flaps vs. Wave Period (s)

Comparison WindFloat and flap-WWF

The three-flaps-WWF base case is compared to the WindFloat base case on Figure 44.

The main influence of the flaps turns out to be on the surge of the platform, which is slightly increased when the flaps are mounted on the platform. This is due to the fact that the flaps increase the area of the platform in contact with the waves. 

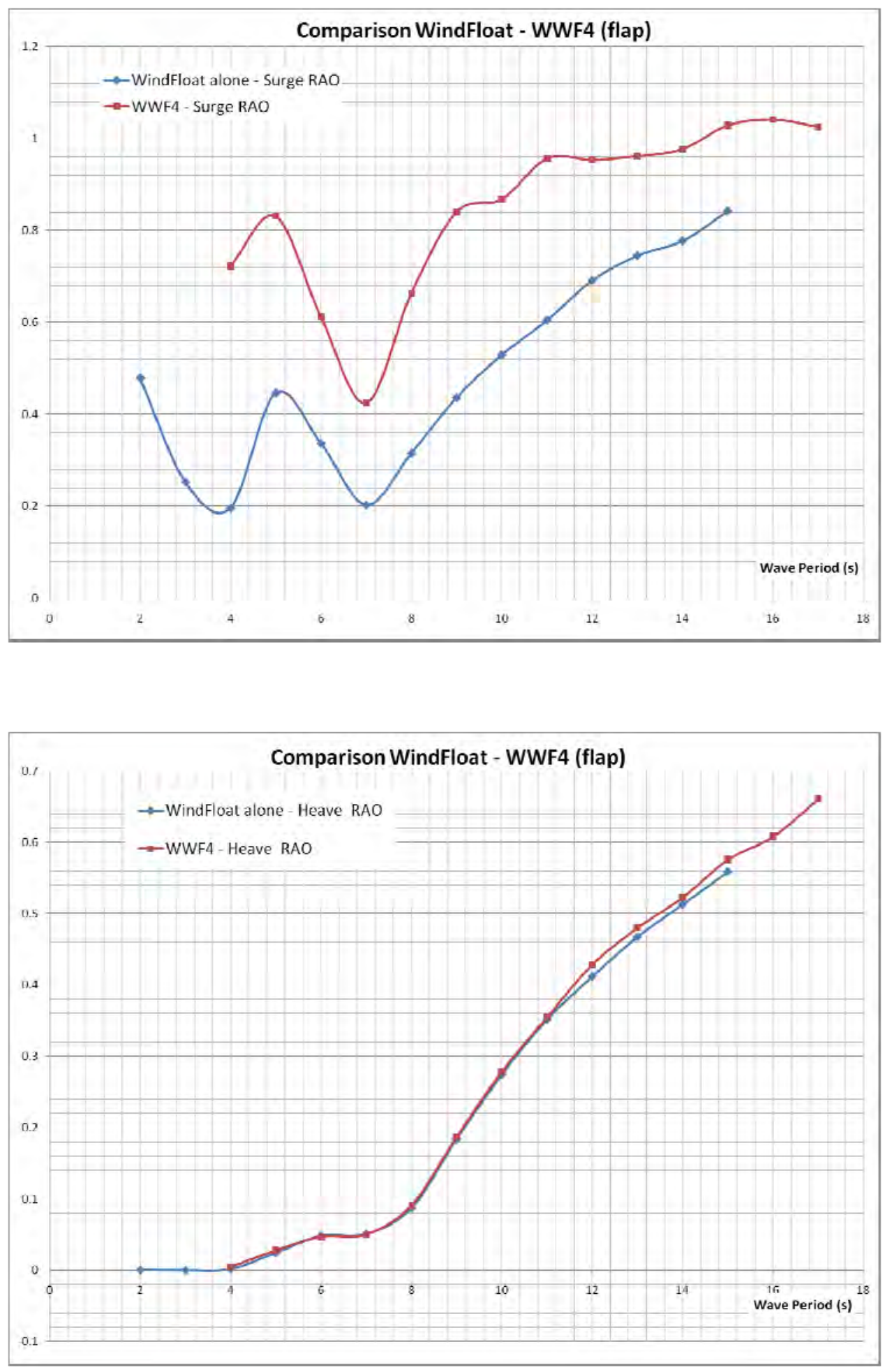


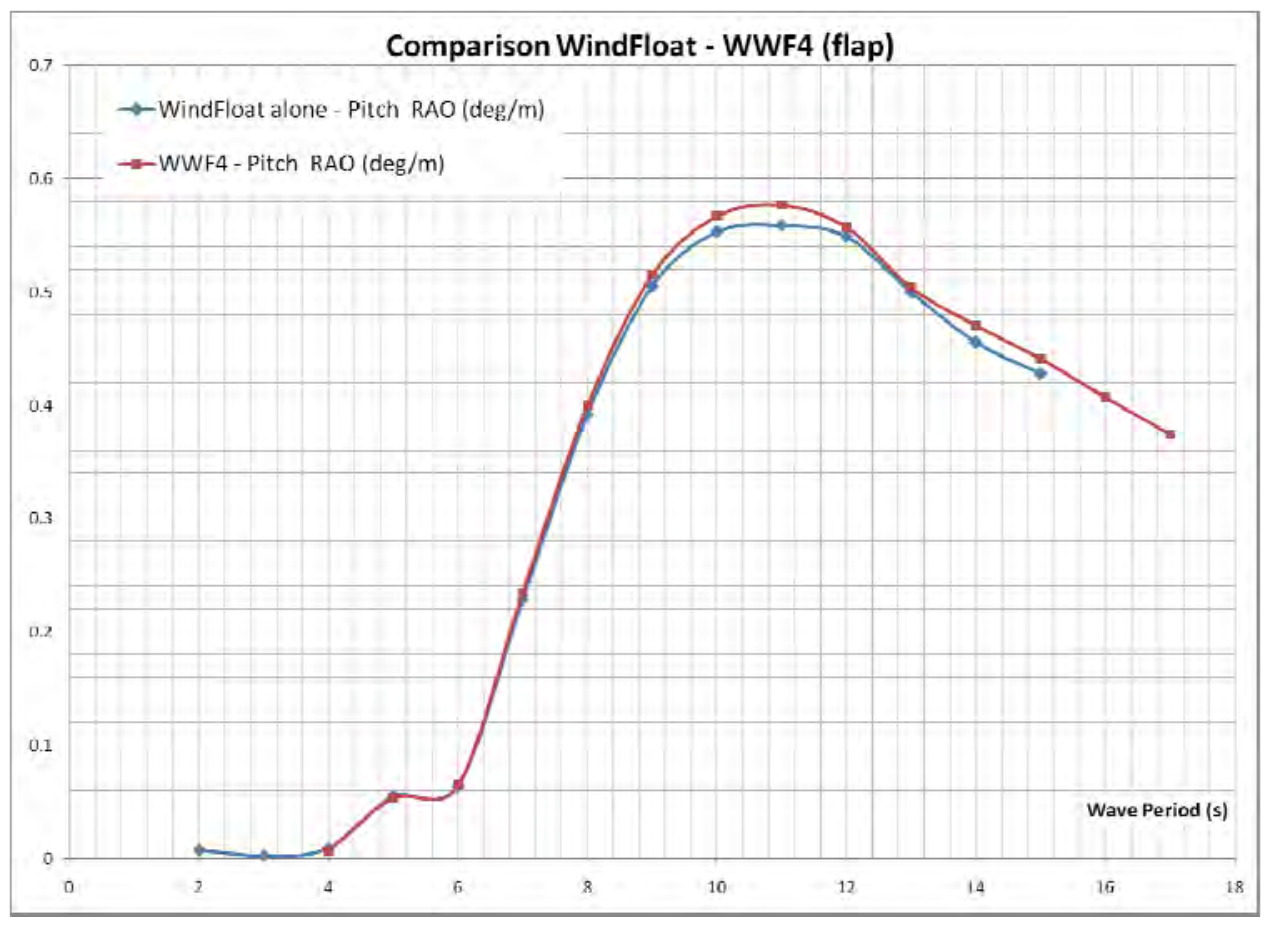

Figure 44a, b, and e: Comparison of the WindFloat RAOs vs. the 3-flaps WWF RAOs for Surge(a), Heave (b), and Pitch (c).

\section{Power curves}

The results of the numerical simulations are presented in Figure 45. The total power per wave amplitude squared is given for the six lines representing the power take-off system, for different damping levels (one color per damping) with respect to the wave period.

The conclusions are the following:

- $\quad$ The maximum power of about $150 \mathrm{~kW} / \mathrm{m}^{2}$ is always obtained at a wave period of 5-6s, which corresponds to the flap pitch resonance period.

- The downwave flap (flap1), directly perpendicular to the incoming waves, has power performances usually higher than the upwave flaps.

- $\quad$ The higher the wave amplitude is, the more the heave resonance period of $6 \mathrm{~s}$ comes into play. This can also be due to the following effect: the stiffness of the lines decreases when the pitch motion amplitude increases (for higher wave amplitudes), and thus the resonance period increases. 

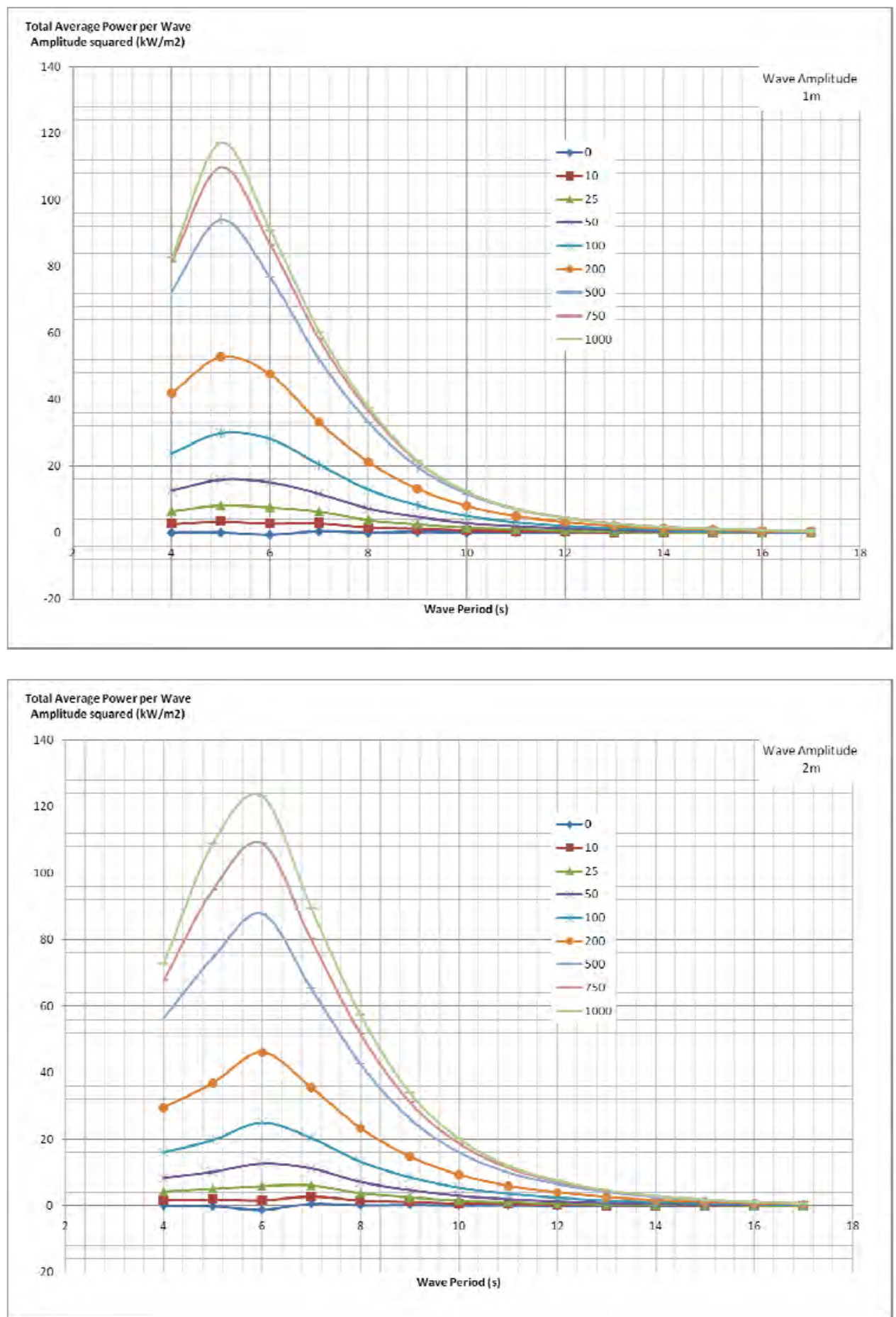


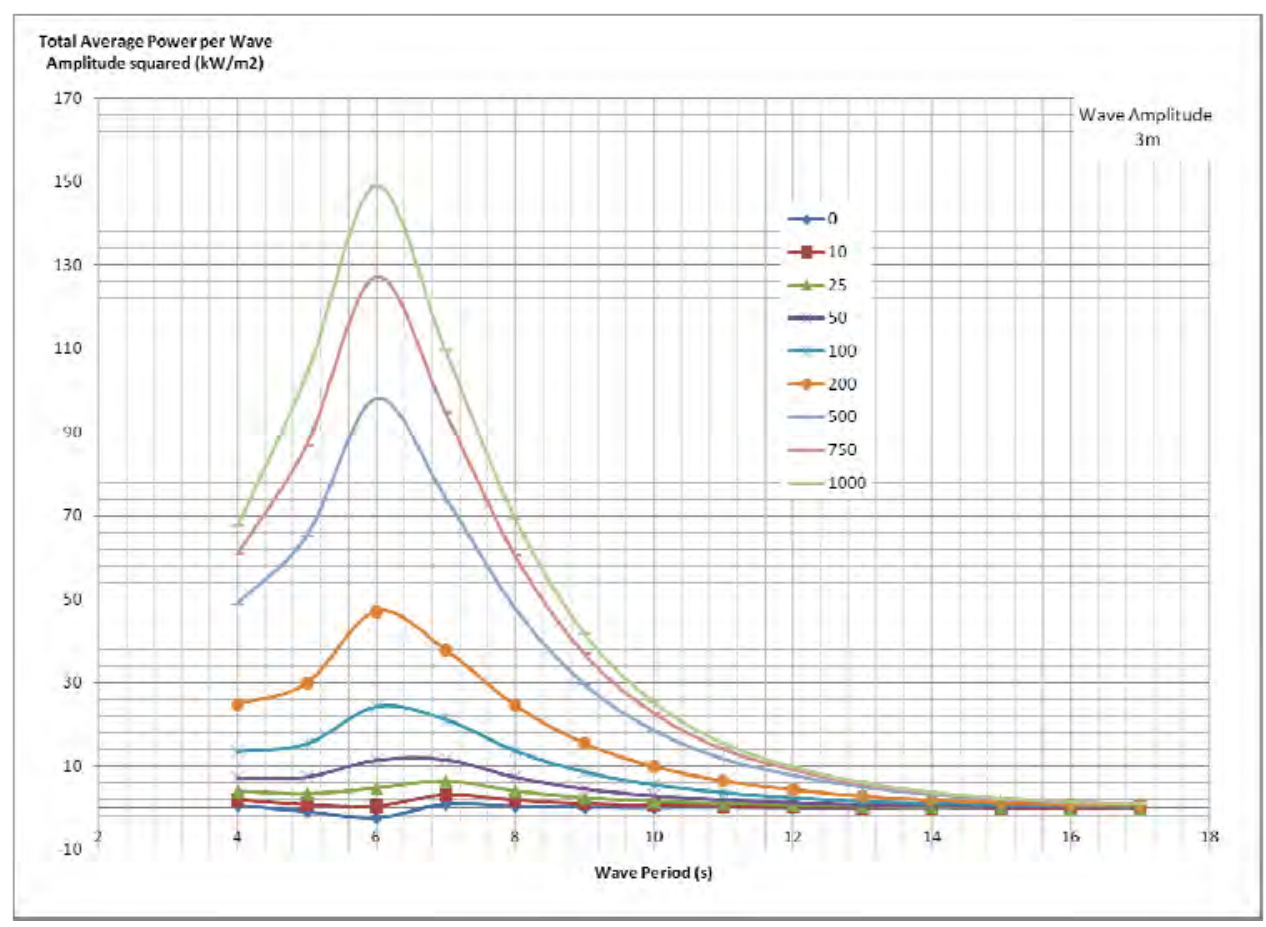

Figure 45 a, b, and c: Total Average Power per Wave Amplitude squared vs. Wave Period, for wave amplitudes of $1 \mathrm{~m}-2 \mathrm{~m}$ and $33 \mathrm{~m}$, and for 9 different line dampings 


\section{Conclusions}

OWC:

A model was developed to incorporate the specificities of the WindFloat platform in the calculation of the power converted by the turbine.

The effect of global platform motion on the performance of the OWC is limited in the range of operational wave periods according to these preliminary results.

The current numerical model neglects non-linear loads on the platform, as well as turbine damping on the internal water free surface. Losses in the turbine rotor and generator are also neglected. Model tests and further analysis are needed to assess the importance of such losses.

The pneumatic efficiency of the turbine however was approximated with a modeled function of flow ratio which is meant to represent the Wells turbine. This model shows that turbine characteristics are essential in the smoothing and optimization of the power output. Different turbine parameters should be tested to optimize the design.

\section{Point absorbers and flaps:}

The different modeled designs hardly affect the WindFloat platform motion. Oscillating plates have the most significant impact by increasing the surge motion amplitude of the WindFloat platform. No showstopper regarding the motion of the WindFloat platform has been discovered during these numerical simulations. Structurally, the effect of the loading of the wave device on the WindFloat hull has yet to be investigated.

Initial predictions show that a maximum average power per wave amplitude squared of $150 \mathrm{~kW} / \mathrm{m}^{2}$ would be potentially harnessed. These performances occur with regular sinusoidal incoming waves at a zero-degree heading, and might decrease substantially with irregular incoming waves. These performances are most likely lower in reality, since shielding effects, radiation effects, and hydrodynamic interferences are not modeled here. Moreover, losses in the PTO systems are not taken into account. These values have not been validated by model tests and some empirical coefficients will need to be adjusted, hence the uncertainty in the absolute value is significant. However, the conclusions based on relative observations can be used to optimize the WEC devices.

It is premature to compare the devices at this stage, since the numerical models need to be validated by part-scale model tests. However, the spherical floater, the three cylindrical floaters, and the three oscillating plates seem to yield similar power performances. 


\section{References}

[1] Principle Power inc, WindWavefloat, " Wave energy pto state-of-the-art report”, Work PACKAGE II DELIVERABLES, DOE GRANT DE-EE0002652

[2] J. Jonkman, T. Larsen, A. Hansen, J. Staerdahl, I. Fylling, M. Karimirad, Z. Gao, T. Moan T. Nygaard, K. Maus J. Nichols .M. Kohlmeier, T. Kossel, W. Zielke J. Pascual Vergara, D. Merino J. Azcona Armendariz, "Offshore Code Comparison Collaboration within IEA Wind Task 23: Phase IV Results Regarding Floating Wind Turbine Modeling", 2010 European Wind Energy Conference (EWEC), 20-23 April 2010, Warsaw, Poland

[3] J. Jonkman, S. Butterfield, W. Musial, AND G. SCOtT, "DeFinition of A 5-MW Reference Wind TURbine for Offshore System DeVelopment," NREL Report No. TP-500-38060, February 2007.

[4] D. Roddier, C. Cermelli, A. Aubault, and A. Weinstein WindFloat: A floating foundation for offshore wind turbines, JOURNAL OF RENEWABLE AND SUSTAINABLE ENERGY 2, 2010

[5] WAMIT USER MANUAL, WAMIT ${ }^{\circledR}, 2006$

[6] C.-H. Lee, WAMit Theory Manual, MIT, 2005

[7] H. Martins-rivas, "Power extraction of an Oscillating Water Column along the Coast", RESEARCH THESIS, MIT, JUNE 2008

[8] L.M.C. Gato and A.F. de O. Falcăo, "Aerodynamics of the Wells turbine”, int. J. Mech. Sci., VOL. 30, No.6, PP.383-395

[9] Curran R., Stewart T.P. and WhitTaker T.J.T., "Design Synthesis of Oscillating Water Column Wave Energy Converters: Performance Matching", Proc. Instn Mech Engrs, Vol.211, Part A, pp.489505

[10] Ragunathan S., "The Wells Air turbine for Wave Energy Conversion", Prog. Aerospace Scl., VOL.31, PP. 335-386 
A Hydrodynamic Modeling with WAMIT

\section{A.1. Modeling and Tuning of the Base Case}

\section{A.1.1. Geometry Discretization}

A high order geometry is generated by creating an analytical representation of the WindFloat components in WAMIT library GeomXact. It includes the columns and the main beams as surfaces and the water entrapment plates are represented as dipoles. For simplicity, the water entrapment plates are circular. The radius of the water entrapment plate is defined so that its surface area is preserved.

This high order geometry is validated against a low order discretization of the same body. The discretization is carried out using the meshing capabilities of Rhino3D. The submerged surfaces are meshed with quadrangular and triangular faces.

The RAOs were compared. No significant difference was found between the two models

For the high order geometry, a convergence analysis is carried out with varying panel sizes. Panel size $\mathrm{PS}=2 \mathrm{~m}$ is chosen.
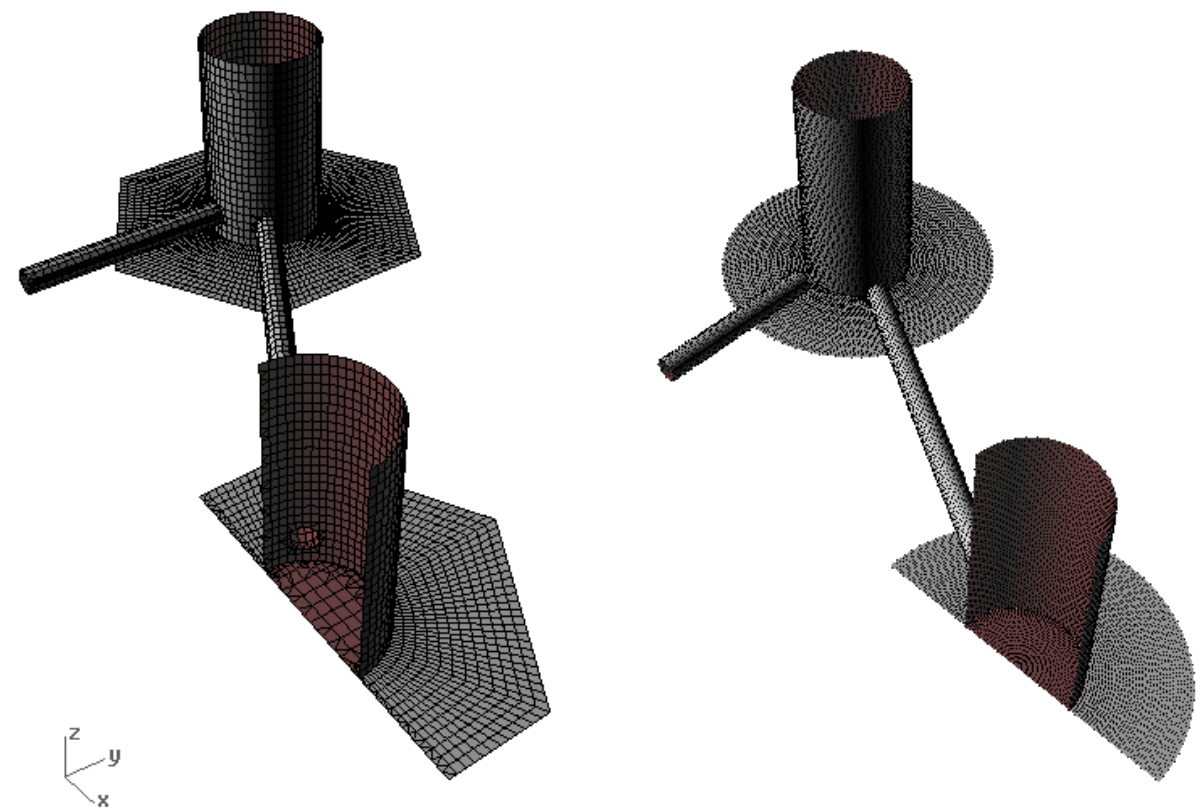

Figure 46: Low Order Discretization of the WindFloat [left] vs High Order Discretization with GeomXact [right] 


\section{A.1.2. Modeling of the Mooring System in WAMIT}

In WAMIT, non-linear effects of the mooring are neglected. Instead, a $6 \times 6$ equivalent stiffness matrix may be used to model the mooring stiffness in surge, sway and yaw.

The stiffness of the system in any direction $\mathrm{i}$ is computed using the following expression:

$T_{i}=2 \pi \sqrt{\frac{M+M_{a, i}}{C_{i}+K_{i}}}$ where $\mathrm{T}_{\mathrm{i}}$ is the period of resonance of the system in direction $\mathrm{i}, \mathrm{M}$ is the mass or inertia, $M_{a, i}$ is the added mass or added inertia in direction $I$ at the period of resonance, $C_{i}$ is the hydrostatic stiffness and $\mathrm{K}_{\mathrm{i}}$ is the equivalent mooring stiffness. The period of resonance in the horizontal plane is determined using decay tests on a detailed numerical model of the mooring system in Orcaflex.

Error! Reference source not found. provides the details of the calculation.

Table 13: Calculation of Equivalent Mooring Stiffness

\begin{tabular}{|c|c|c|c|c|c|}
\hline & $\begin{array}{c}\text { Period of } \\
\text { Resonance } \\
{[s]}\end{array}$ & $\begin{array}{l}\text { Added Mass } \\
\quad[\mathrm{kg}] \text { or } \\
\text { Inertia }\left[\mathrm{kg} \cdot \mathrm{m}^{2}\right]\end{array}$ & $\begin{array}{c}\text { Mass [kg] } \\
\text { or } \\
\text { Inertia }\left[\mathrm{kg} \cdot \mathrm{m}^{2}\right]\end{array}$ & $\begin{array}{c}\text { Hydrostatic } \\
\text { Stiffness } \\
{[\mathrm{N} / \mathrm{m} \text { or } \mathrm{N} . \mathrm{m} / \mathrm{rad}]}\end{array}$ & $\begin{array}{c}\text { Equivalent } \\
\text { mooring stiffness } \\
{[\mathrm{N} / \mathrm{m} \text { or } \mathrm{N} . \mathrm{m} / \mathrm{rad}]}\end{array}$ \\
\hline Surge & 108.6 & $4.10 \mathrm{E}+06$ & $4.64 \mathrm{E}+06$ & $0.00 \mathrm{E}+00$ & $29,270.0$ \\
\hline Sway & 135.7 & 4.10E+06 & $4.64 \mathrm{E}+06$ & $0.00 \mathrm{E}+00$ & $18,730.0$ \\
\hline Yaw & 71.3 & $2.76 \mathrm{E}+09$ & $3.26 \mathrm{E}+09$ & $0.00 E+00$ & $4.677 \mathrm{E}+07$ \\
\hline
\end{tabular}

\section{A.1.3. Modeling of Viscous damping}

WAMIT over-predicts the resonance in heave, roll and pitch. In reality, viscous damping on the water entrapment plates limits the amplitude of motion at the resonance.

To model the viscous effect, an equivalent linear damping is provided in heave, roll, and pitch.

The equivalent damping is a fraction of critical damping $\beta=2 \sqrt{\left(M+M_{a}\right) \times C_{i}}$. The critical damping in heave, roll and pitch is provided in Table 14. To determine what fraction of critical damping should be used in the model, a sensitivity analysis is carried out. WAMIT results are plotted for pitch motion in Figure 47 for damping ratios ranging between 10 and 20\%. Results are compared with the RAOs obtained with the Orcaflex model of the WindFloat.

$10 \%$ damping ratio in heave, pitch and roll, which matches best the Orcaflex results, is retained for further analysis. 
Table 14: Computation of critical damping

\begin{tabular}{|l|r|r|r|r|r|}
\hline & $\begin{array}{c}\text { Period of } \\
\text { Resonance } \\
{[\mathrm{s}]}\end{array}$ & $\begin{array}{c}\text { Added Mass } \\
{[\mathrm{kg}] \text { or Inertia }} \\
{[\mathrm{kg} . \mathrm{m} 2]}\end{array}$ & $\begin{array}{c}\text { Mass }[\mathrm{kg}] \text { or } \\
\text { Inertia } \\
{\left[\mathrm{kg} \cdot \mathrm{m}^{2}\right]}\end{array}$ & $\begin{array}{c}\text { Hydrostatic } \\
\text { Stiffness [N/m or } \\
\text { N.m/rad] }\end{array}$ & $\begin{array}{c}\text { Critical } \\
\text { Damping }\end{array}$ \\
\hline Heave & 19.9 & $1.90 \mathrm{E}+07$ & $4.64 \mathrm{E}+06$ & $2.37 \mathrm{E}+06$ & $1.5 \mathrm{E}+07$ \\
\hline Roll & 43.3 & $7.76 \mathrm{E}+09$ & $5.72 \mathrm{E}+09$ & $2.83 \mathrm{E}+08$ & $3.9 \mathrm{E}+09$ \\
\hline Pitch & 43.2 & $7.76 \mathrm{E}+09$ & $5.65 \mathrm{E}+09$ & $2.83 \mathrm{E}+08$ & $3.9 \mathrm{E}+09$ \\
\hline
\end{tabular}
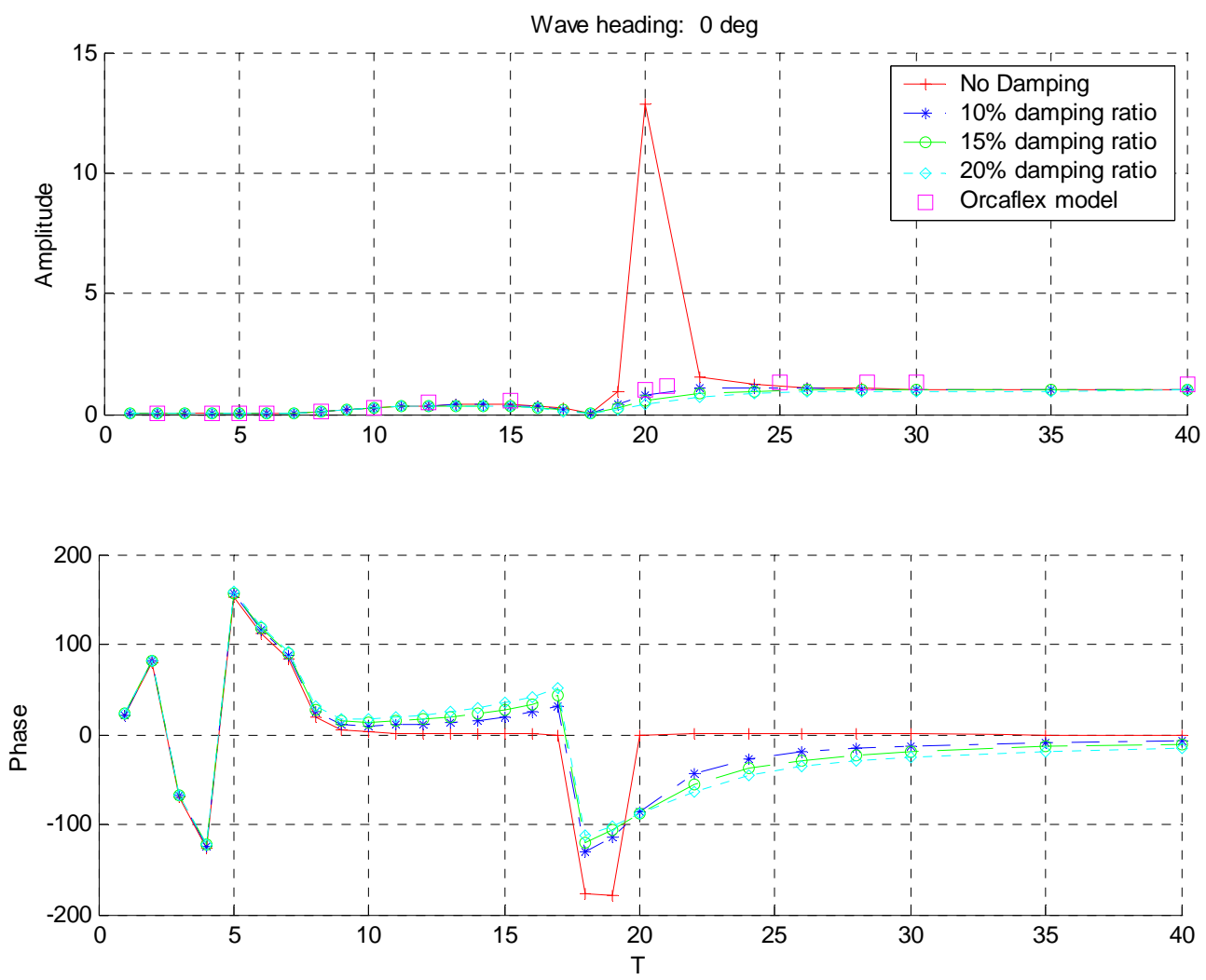

RAO in direction 3

Figure 47: Effect of Equivalent Damping on Heave RAO (amplitude in m/m) 


\section{A.2. Model of Oscillating Water Column}

\section{A.2.1. GeomXact Routine for automation of geometry generation}

A Fortran routine was written for the WAMIT library to automate the generation of the OWC geometry. It is based on the routine used for the WindFloat hull. The OWC draft and diameter are added as parameters and a patch id added to define the submerged part of the outer cylinder.

\section{A.2.2. Wave Elevation in the Wave Field and on the Internal Surface}

The wave field is discretized and the wave elevation is computed at each point, as shown in Figure 48 for $D_{\text {owc }}=14 \mathrm{~m}$. About 1000 points are used to represent the $40 \mathrm{~m} \times 40 \mathrm{~m}$ wave field. The area around the columns is refined to capture local variations. Several level of refinements are tested to ensure the details are well represented.

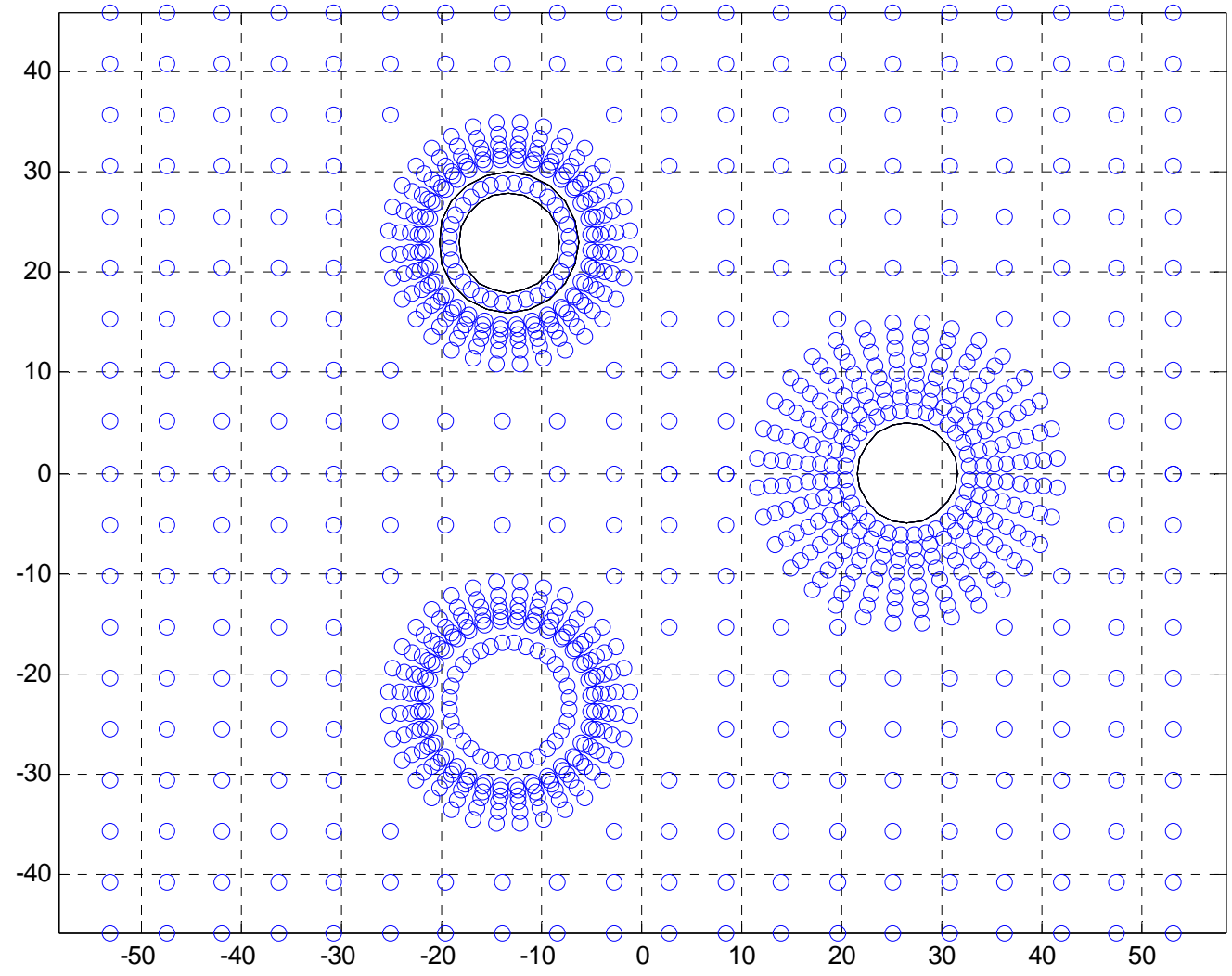

Figure 48: Wave Field Discretization (Circle = field point for wave elevation calculation)

A convergence analysis is carried out to ensure the panel size on the geometry is small enough to represent the wave elevation accurately. Results are shown for $T_{\text {owc }}=3 \mathrm{~m}$ and $D_{\text {owc }}=14 \mathrm{~m}$ in Figure 49 . A panel size of $2 \mathrm{~m}$ is used. 

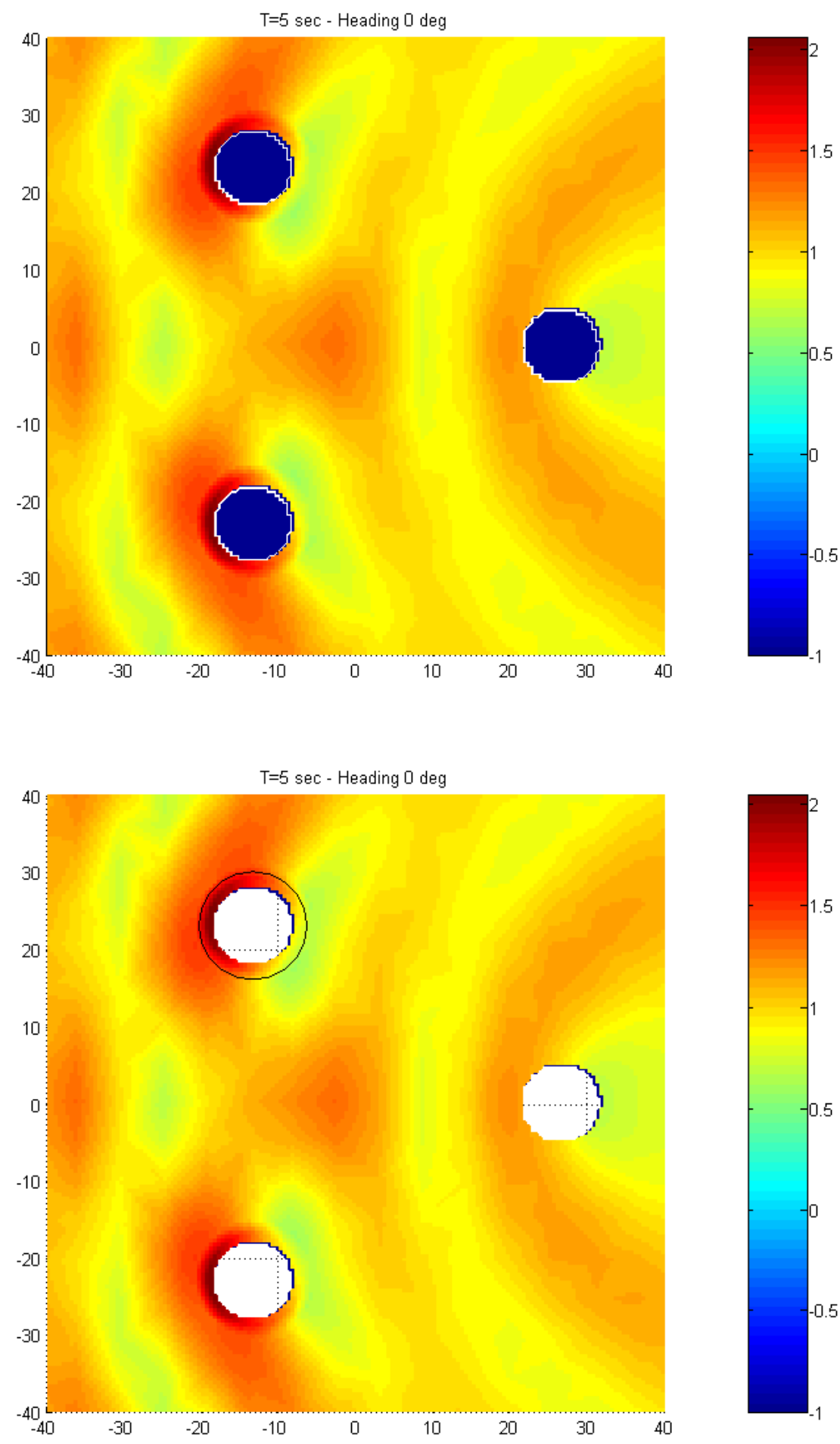

Figure 49: Map of Amplitude of Wave Elevation $[\mathrm{m} / \mathrm{m}]$ for panel size $=2 \mathrm{~m}$ [top] or panel size $=1 \mathrm{~m}\left[\right.$ bottom] $-\mathrm{T}_{\mathrm{owc}}=3 \mathrm{~m}, \mathrm{D}_{\mathrm{owc}}=14 \mathrm{~m}$, Wave Period $\mathrm{T}=5 \mathrm{sec}$ 
A convergence analysis is carried out on the number of points around the circumference of the internal free surface. In this analysis, for $D_{\text {owc }}=14 \mathrm{~m}, 32$ points are used around the annulus of the chamber to define the internal free surface as shown in Figure 50. This is sufficient to obtain a good approximation of power for periods larger than 3s.

In the radial direction, only 1 point is used at this diameter, since field points must be at least $1 / 2$ a panel size away from the wall for convergence of the WAMIT solution. For larger diameters, additional points are added as needed.

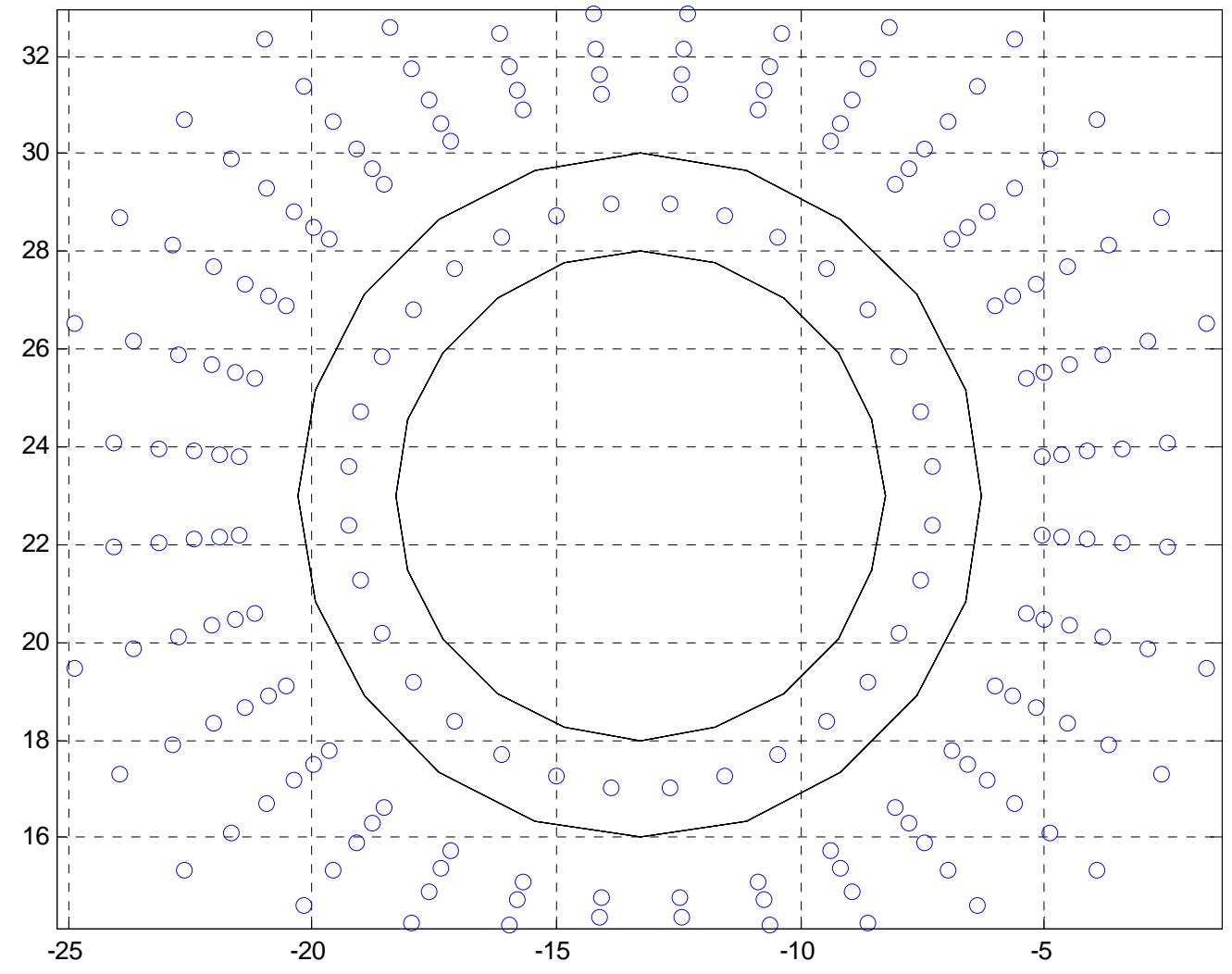

Figure 50: Discretization of internal free surface with field points 
Appendix C: Model Testing and Numerical Tool Validation of Wave Devices Fitted into the WindFloat 


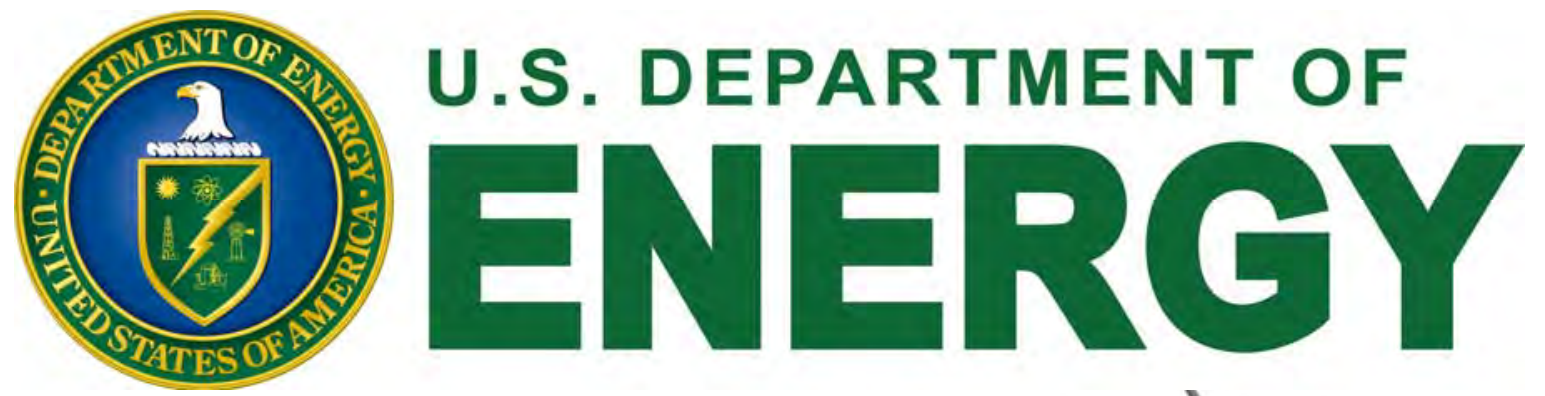

DOE Grant to Support the Development of Adyanced Water Power Technologies

\section{WindWaveFloat (WWF)}

By:

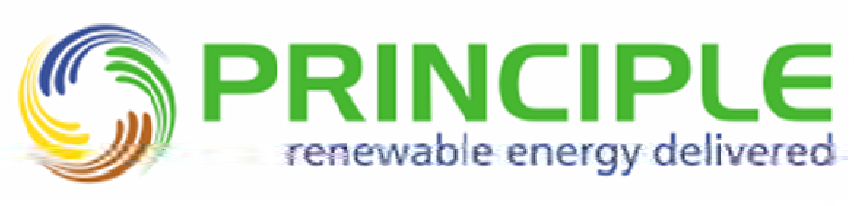

WWF CONCEPT DESIGN DELIVERAB LE \#

Model Testing and Numerical Tool Validation of Wave Devices fitted into the WindFloat

3rd Quarter Reporting

January 2011 


\section{Table of Contents}

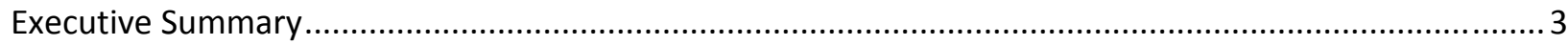

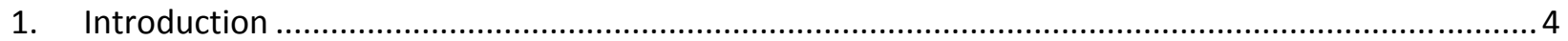

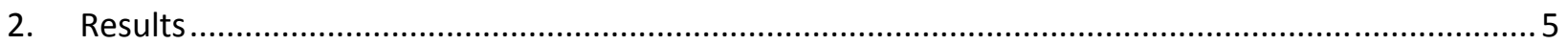

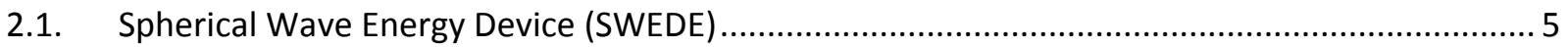

2.1.1. Validation of the numerical models ............................................................................. 5

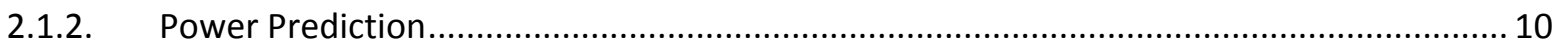

2.2. Oscillating Wave Surge Converter (OWSC or FLAPS) …..................................................... 15

2.2.1. Validation of the numerical models ......................................................................... 15

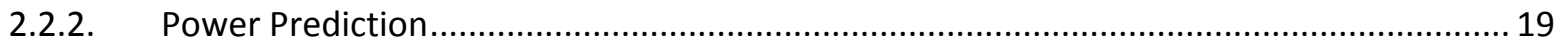

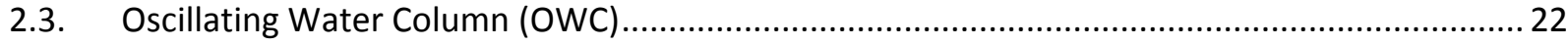

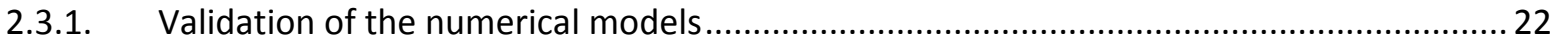

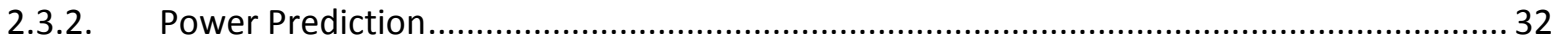

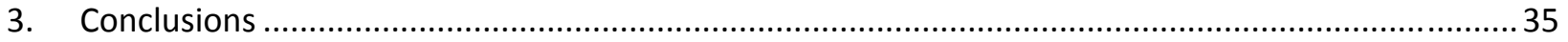

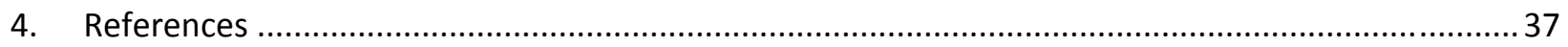

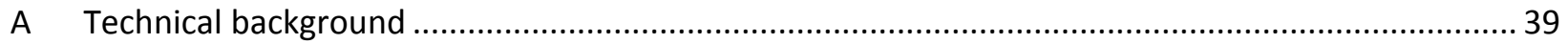

A.1. OMAE2011-50278: A Generic 5 MW WindFloat for Numerical Tool Validations....................... 39

A.2. OMAE2011-49014: A Generic 5 MW WindFloat for Numerical Tool Validations....................... 39

A.3. OMAE2011-49015: Design of a Point Absorber inside the WindFloat Structure ...................... 39 


\section{Executive Summary}

This report summarizes the WindWaveFloat model tests that were performed at the University of California Berkeley Towing Tank Facility between October 18 and November 5, 2010. These tests were designed to provide confidence in the numerical tools developed in the previous task of this grant.

The numerical models were slightly modified to make sure that discrepancies are due to modeling inaccuracies, rather than differences between the numerical and experimental model. In these tests, three different wave-energy devices were tested.

- The Spherical Wave Energy Device, (SWEDE), a large floater placed inside the triangle and connected to the three columns. Power production comes from the relative motion between the SWEDE and the WindFloat

- The Oscillating Wave Surge Converter (OWSC) hinged on the WindFloat main beams. The oscillating plates drive a rotation of the main axis, which is converted into energy. A flywheel, located inside the top of column, is used to smooth out the energy production, but is not modeled in the simulations yet.

- The Oscillating Water column (OWC), being a large shell around column 2 and 3, and a Wells turbine producing energy from the flux of compressed air in the turbine

In the previous report, a fourth concept was analyzed. The multiple-point-absorber device is similar in nature with the SWEDE, so it was felt unnecessary to model test it at this stage, as the learning from the SWEDE can be applied directly to this device.

The WindFloat without any of the three wave-energy converters (WECs) was also tested to verify the predicted motions. In the experiments, the WindFloat motions and sufficient terms were measured to predict the mechanical power out of the WECs. By comparing the motions of the WindFloat with and without the WEC, the influence of the WECs on the hull can be estimated. Those results are presented herein, both numerically and experimentally. This report also presents the optimized amount of power that each WEC can harness in regular waves, by varying different parameters.

The next phase of this work is to optimize the amount of power that each device can harness in irregular waves. A basic structural analysis will also be performed to understand the cost associated in outfitting the WindFloat hull to support the WEC. From this data, for a single unit, the power to cost ratio of the WindFloat and of each WindWaveFloat configurations will be assessed. 


\section{Introduction}

Under DOE award DE-EE0002652, Principle Power is studying the effect of adding wave-energy device(s) to the WindFloat hull. The study is framed around understanding the economic impact of adding an additional source of energy production, at a lower cost than in their stand-alone forms, since mooring, electrical infrastructure, and other components are being shared with the existing WindFloat.

Earlier reporting $\left(2^{\text {nd }}\right.$ quarter reporting) described the numerical models that were developed to study the four generic configurations that were chosen, based on existing concepts, industry practices, and synergies in the integration into the WindFloat hull.

These models are validated against experiments conducted at the University of California, Berkeley towing test facility in the fall 2010. Then using the validated models, a power prediction is performed.
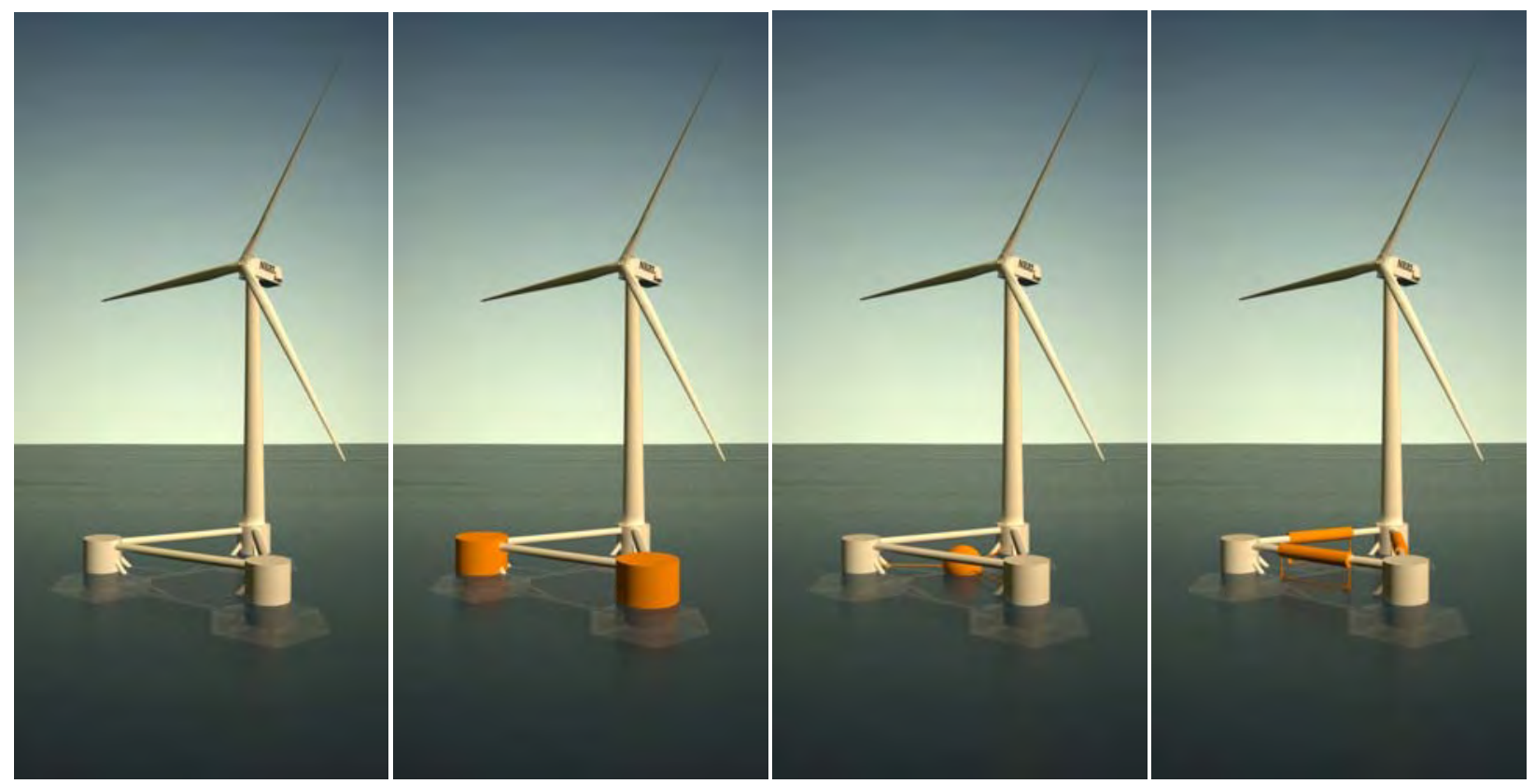

Figure 1: WindWaveFoat configurations (Base case, OWC, SWEDE, OWSC)

This report presents the validated results in terms of motion and power. Details on the setup of the experiments are not document herein, in order to keep the document succinct, and clear. More details on the experimental setup can be found in the published articles inserted in the appendices A.2 and A.3. 


\section{Results}

In this section, for each WEC presented in this study, the validation of the numerical models on motions, the influence of each device on the WindFloat platform (and the converse when possible), and the predictions of average mechanical power output and capture width in regular waves are summarized.

\subsection{Spherical Wave Energy Device (SWEDE)}

\subsubsection{Validation of the numerical models}
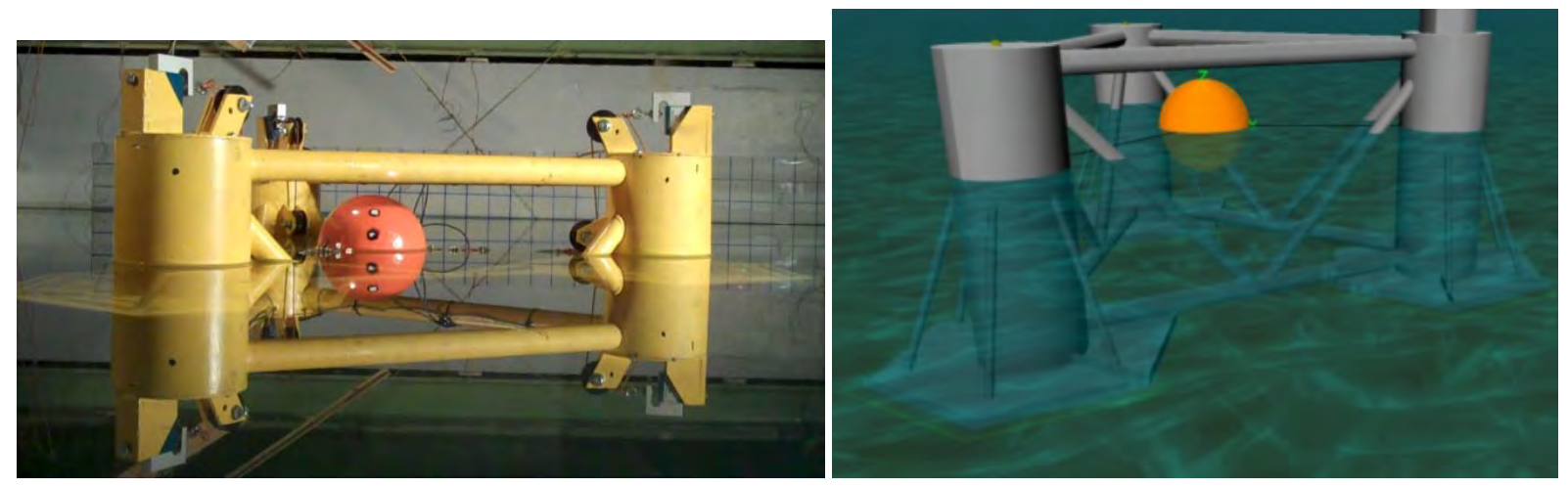

Figure 2 a,b: Picture of the WindWaveFloat-SWEDE model in the UC Berkeley towing tank and side view of OrcaFlex WindWaveFloat numerical model incorporating the SWEDE.

The WindFloat platform numerical model validation is described in the $2^{\text {nd }}$ quarter report. Only the SWEDE numerical models and the WindWaveFloat numerical models are discussed in this section. The SWEDE damping coefficients and drag coefficients in surge, heave, and pitch are adjusted in the OrcaFlex WindWaveFloat numerical model to match the time-series of decay tests of the SWEDE alone at 1:30, and the RAOs of the WindWaveFloat at 1:78.5 performed during the tank tests.

The SWEDE natural periods are given in Table 1 for the two SWEDE line stiffness coefficients tested in the wave basin ( $27 \mathrm{kN} / \mathrm{m}$ and $140 \mathrm{kN} / \mathrm{m}$ at full scale). The heave natural period does not change because the stiffness of the line barely affects the vertical motion of the sphere, in the small motion approximation.

Table 1: SWEDE natural periods (full scale) (WindFloat platform fixed) based on experimental decay tests

\begin{tabular}{|l|c|c|}
\cline { 2 - 3 } \multicolumn{1}{c|}{} & $\begin{array}{c}\text { Line stiffness coefficient } \\
\text { of } \mathbf{1 4 0} \mathbf{~ k N / m}\end{array}$ & $\begin{array}{c}\text { Line stiffness coefficient } \\
\text { of } \mathbf{2 7} \mathbf{~ k N} / \mathbf{m}\end{array}$ \\
\hline SWEDE Surge natural period (s) & 7.8 & 17.6 \\
\hline SWEDE Heave natural period (s) & 3.7 & 3.7 \\
\hline
\end{tabular}

Three types of results are then presented. First, the experimental RAOs of the WindFloat alone are compared with the experimental RAOs of the WindWaveFloat with the SWEDE to study the impact of 
the SWEDE on the WindFloat platform. Second, the theoretical and experimental RAOs and force RAOs of the SWEDE are included. No damping is added to the lines at this point, because the aim is to validate the numerical models with the experimental tests, and no damping was used in the first series of experimental tests reported here. Third, the RAOs of the SWEDE on a fixed WindFloat are compared with the RAOs on a freely-floating WindFloat to assess the impact on the WindFloat platform on the SWEDE motions.

\section{WindFloat RAOs Comparisons: Impact of the SWEDE on the WindFloat platform}

To understand the impact of the SWEDE on the WindFloat motions, the RAOs of the WindFloat alone and the RAOs of the WindWaveFloat with the SWEDE, both measured experimentally, are compared for the same wave slope on Figure 3, for a line stiffness coefficient of $140 \mathrm{kN} / \mathrm{m}$. The wave slope $W s$ is defined as the ratio of the wave amplitude and the wavelength.

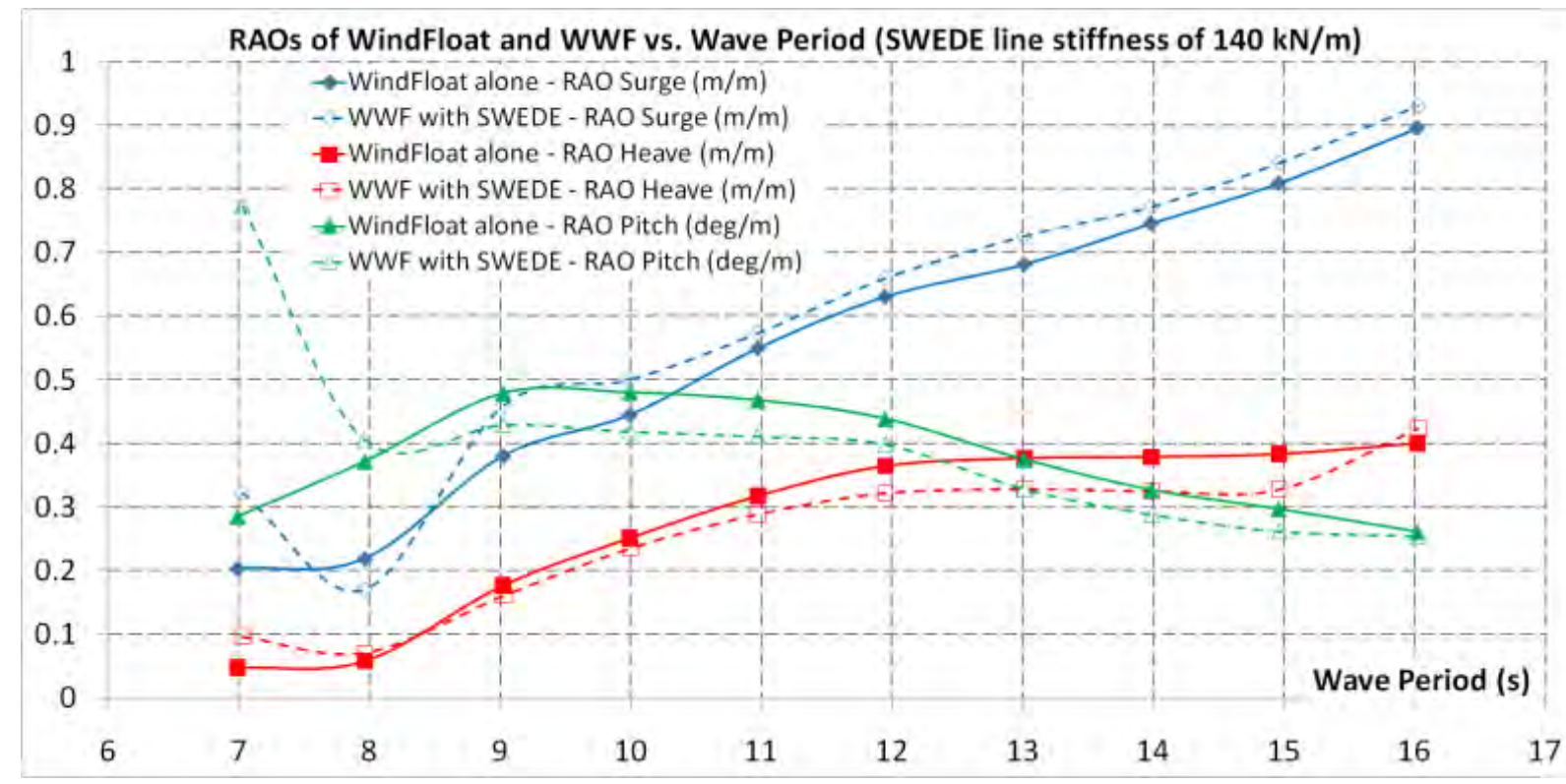

Figure 3: Comparison of the Surge, Heave, and Pitch RAOs of the WWF and the WindFloat alone. The wave slope $W s$ is $6 \%$.

It can be seen that the SWEDE minimally affects the motions of the WindFloat platform. Besides a slight rise of all motions after the heave period of resonance of the SWEDE (between $4 \mathrm{~s}$ and $8 \mathrm{~s}$ ), only the surge motion of the WindFloat is slightly amplified over the range of tested periods. Past the SWEDE surge period of resonance (about $8 \mathrm{~s}$ ), the WindFloat platform heave and pitch motions are even slightly reduced.

The limitation in this comparison of WindFloat RAOs vs. WindWaveFloat RAOs is that results are presented only for given SWEDE line stiffness coefficients and a zero damping coefficient. Future numerical simulations must be performed to make sure that the same conclusions can be drawn for any SWEDE line stiffness and damping coefficients, especially combinations that convert a significant amount of wave power into mechanical power. 


\section{SWEDE Motion RAOs and Force RAOs}

The numerical and experimental SWEDE RAOs are presented in Figure 4 and Figure 5 for two different line stiffness coefficients, $27 \mathrm{kN} / \mathrm{m}$ and $140 \mathrm{kN} / \mathrm{m}$. There is no damping in these simulations to verify the extreme motions.

The SWEDE presents different periods of resonance in surge, heave, and pitch for different line stiffness coefficients. Constrained by the period range allowed by the wave-maker, the RAOs presented on Figure 4 show only periods out of the resonance areas in surge (17.6s), and heave-pitch (3.7s), even if the heave and pitch motions are the highest around $6 \mathrm{~s}$, and the surge motion keeps increasing until $14 \mathrm{~s}$. The match between numerical and experimental results is what is really sought for, and confirmed here.

The period of resonance in surge for a line stiffness coefficient of $140 \mathrm{kN} / \mathrm{m}$ clearly appears at $7.9 \mathrm{~s}$ (Figure 5). Similarly the high pitch motion is induced by the surge resonance, due to the line configuration of the SWEDE. A slight difference in peak amplitude is observed, probably due to a slight miscalculation of hydrodynamic damping. As predicted, the heave motion of the SWEDE is hardly impacted by the line stiffness coefficient, since the lines are attached to the SWEDE horizontally, at the water line level, and the major stiffness coefficient is the hydrodynamic component in this degree-offreedom.

Similarly, the SWEDE Force RAOs, defined as the RAO of the three tensions in the line at its end connected to the spherical floater, is presented in Figure 6 for a line stiffness coefficient of $27 \mathrm{kN} / \mathrm{m}$. Figure 6 confirms the match between the numerical simulations and the experimental results, for both Motion RAOs and Force RAOs. This match gives confidence in the power prediction that will be obtained numerically as it is calculated from the product of the force by the rate of change of motion.

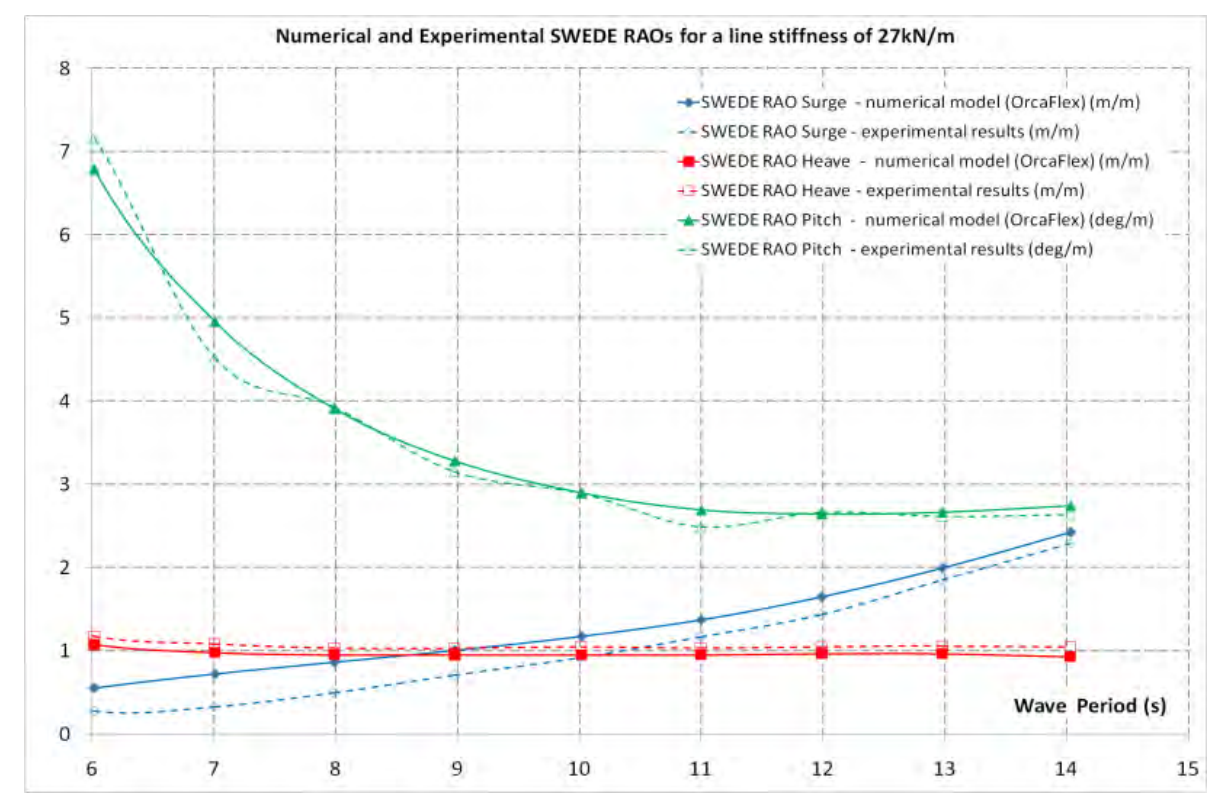

Figure 4: Comparison of numerical vs. experimental SWEDE RAOs on the WindWaveFloat for a line stiffness coefficient of $27 \mathrm{kN} / \mathrm{m}$. The wave slope $W s$ is $6 \%$. 


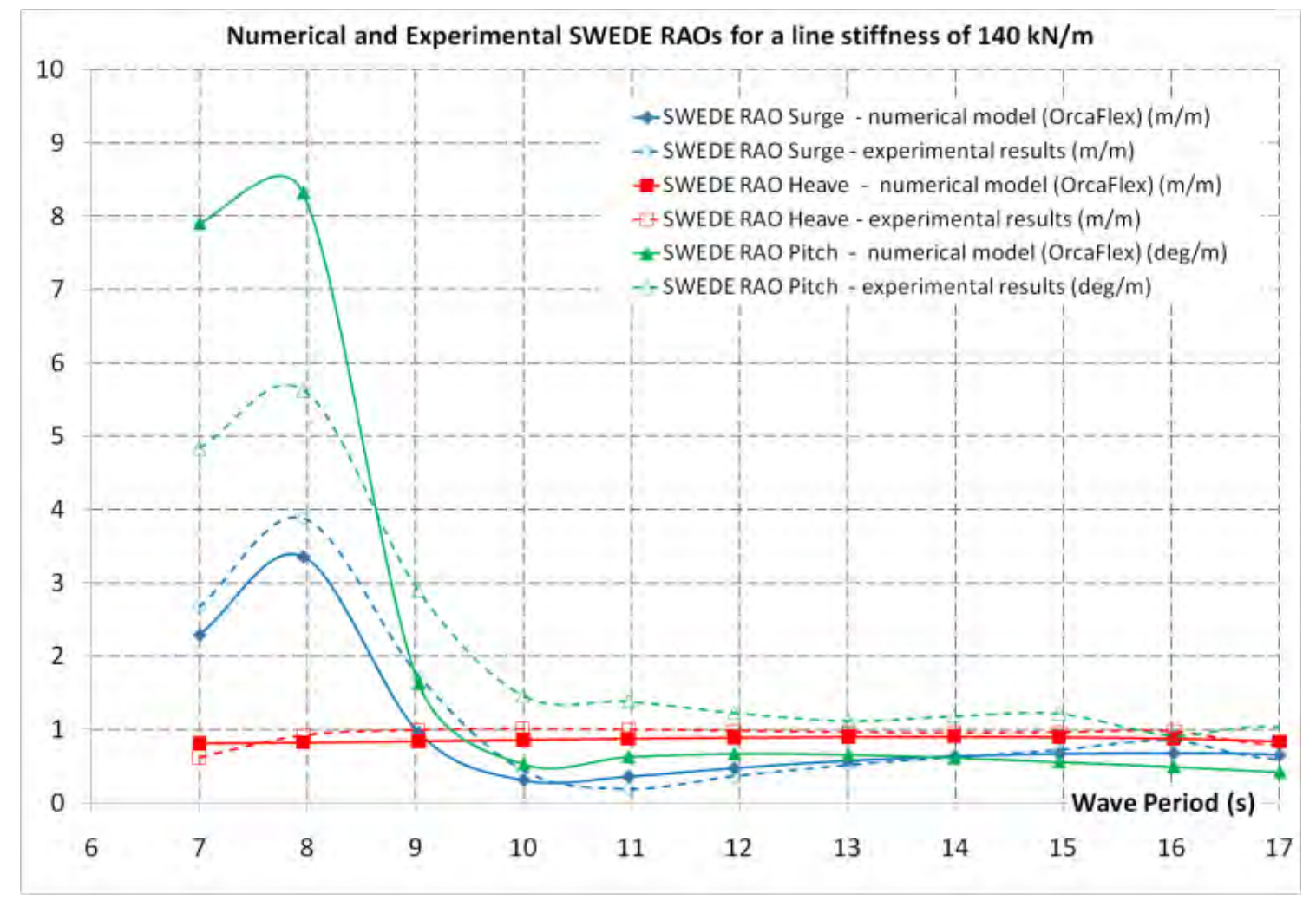

Figure 5: Comparison of numerical vs. experimental SWEDE RAOs on the WindWaveFloat for a line stiffness coefficient of $140 \mathrm{kN} / \mathrm{m}$. The wave slope $W s$ is $6 \%$.

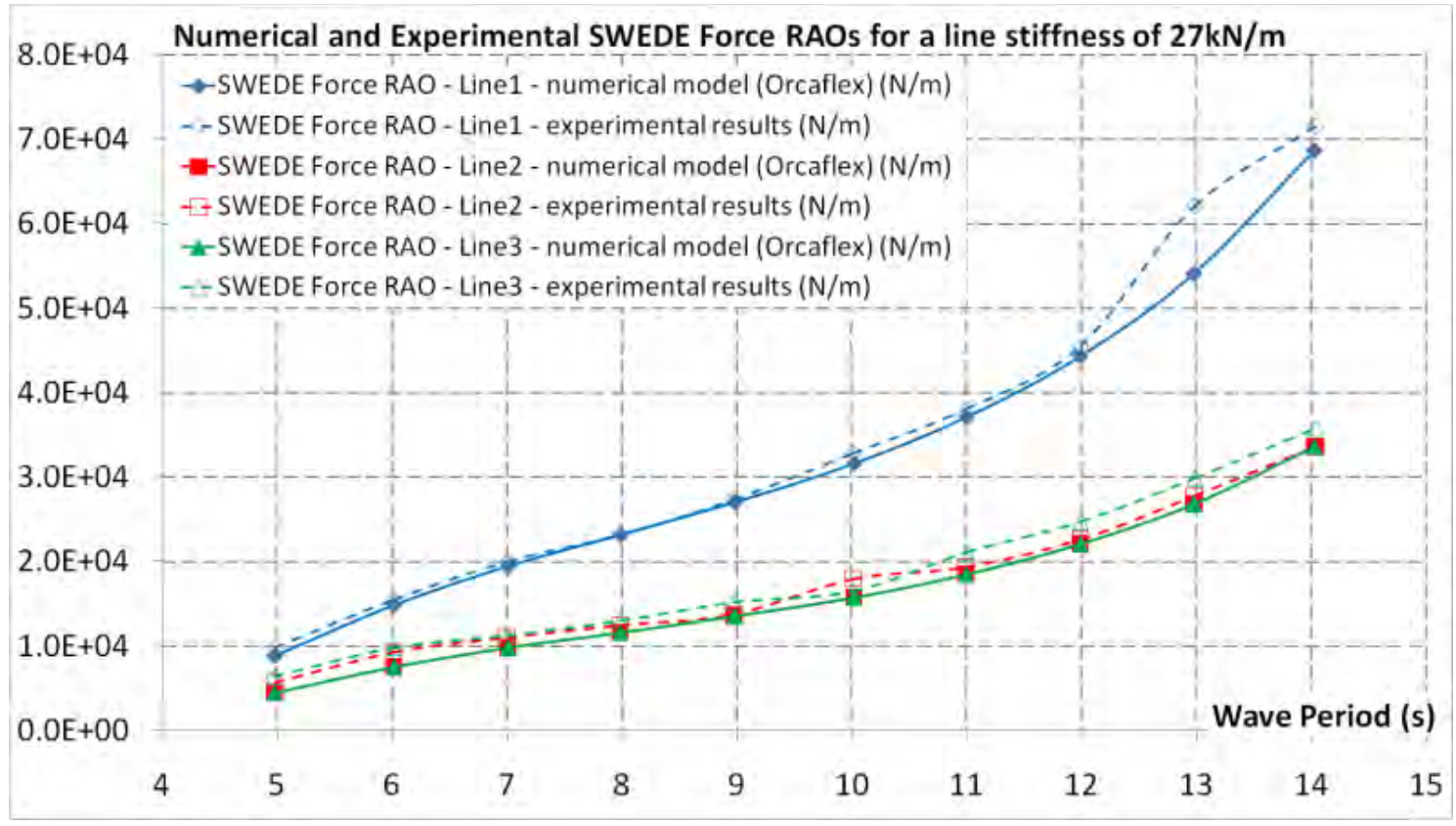

Figure 6: Comparison of theoretical vs. experimental SWEDE Force RAOs for a line stiffness coefficient of $27 \mathrm{kN} / \mathrm{m}$. The wave slope $W s$ is $6 \%$. 
SWEDE RAOs comparisons: Impact of the WindFloat platform on the SWEDE RAOS and Force RAOs

Figure 7 and Figure 8 present examples of the SWEDE Motion RAOs and Force RAOs attached to a fixed WindFloat in comparison with the SWEDE RAOs and Force RAOs in the normal WindWaveFloat configuration (freely-floating WindFloat platform). It is deduced that a freely-moving WindFloat platform slightly reduces the sphere surge and pitch motions, except around the surge resonance period (8s), but significantly reduces the tensions in the lines. A peak in Force RAO for the three lines appears at around 6s. The WindFloat and the sphere happen to be about 180 degree - out of phase at this period of $6 \mathrm{~s}$, thus the relative motion of the SWEDE with respect to the WindFloat is maximal, yielding the highest tensions in the SWEDE lines.

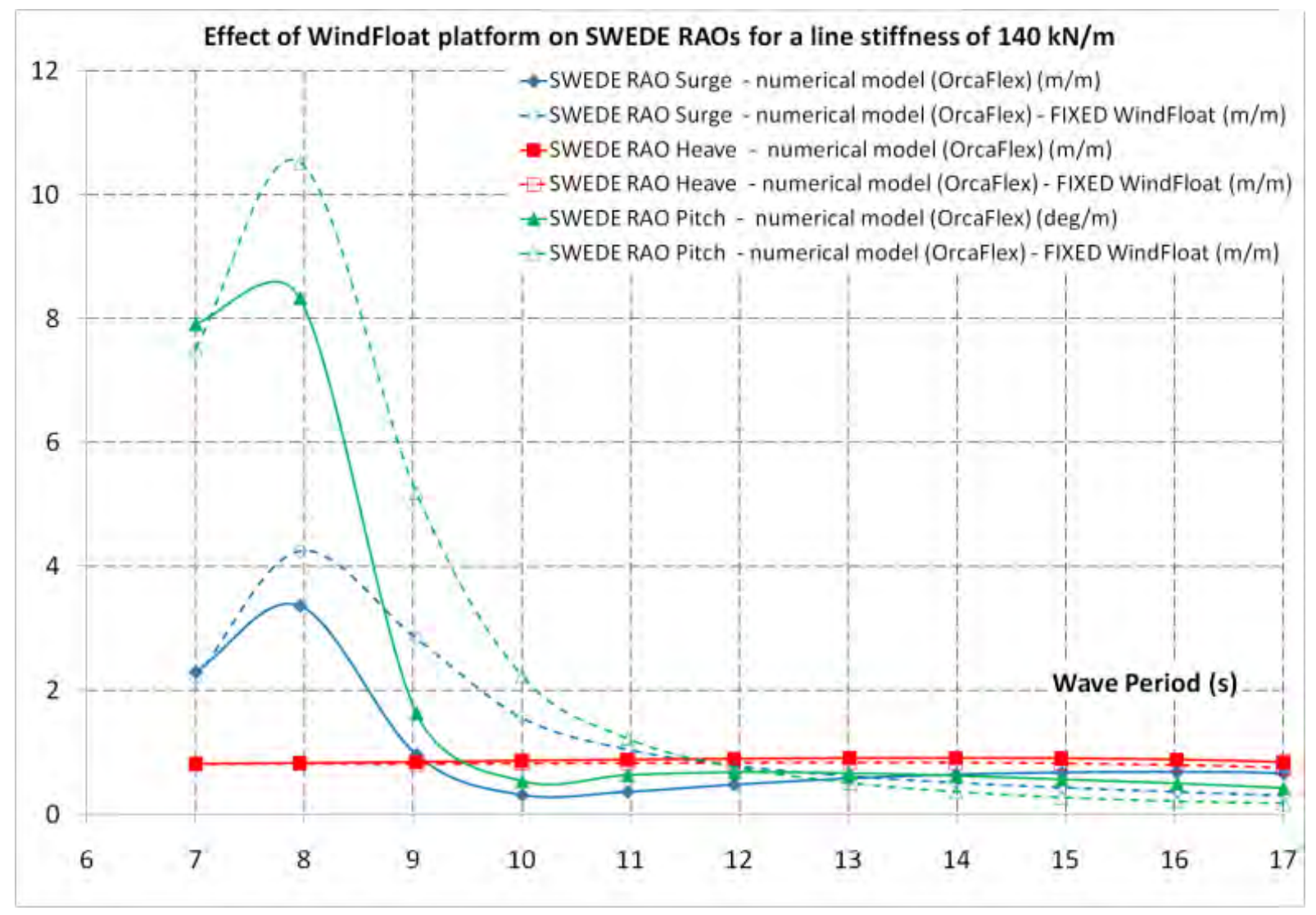

Figure 7: Comparison of numerical SWEDE Motion RAOs on a fixed WindFloat vs. freely floating WindFloat (WindWaveFloat) for a line stiffness coefficient of $140 \mathrm{kN} / \mathrm{m}$. The wave slope $W s$ is $6 \%$. 


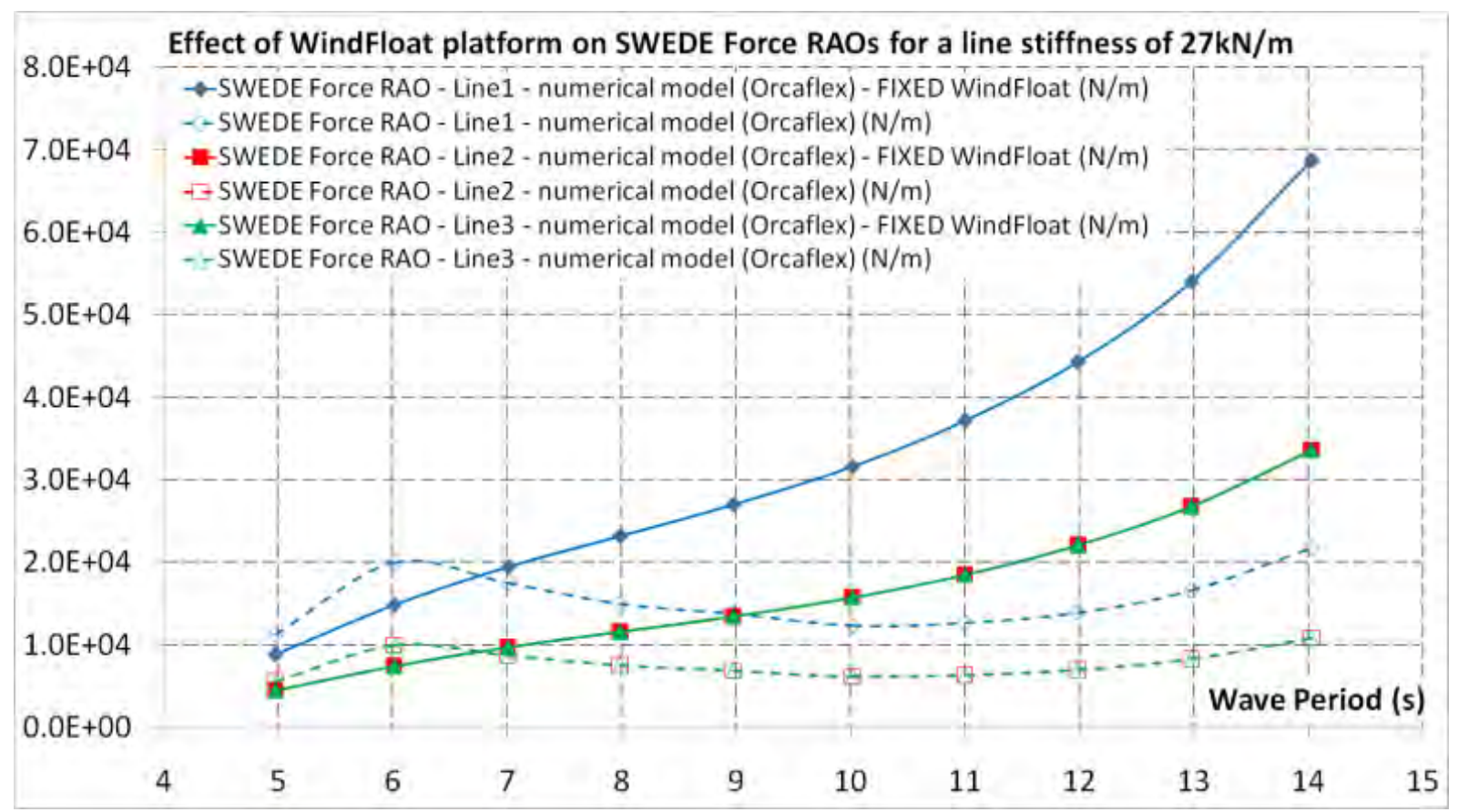

Figure 8: Comparison of numerical SWEDE Force RAOs on a fixed WindFloat vs. freely floating WindFloat (WindWaveFloat) for a line stiffness coefficient of $27 \mathrm{kN} / \mathrm{m}$. The wave slope $W s$ is $6 \%$.

\subsubsection{Power Prediction}

Now that the numerical models are validated, some damping is added in the lines holding the SWEDE, to simulate the presence of a power take-off system. The average mechanical power generated by the SWEDE and the capture width are thus computed for different damping coefficients at the two tested line stiffness coefficients.

\section{Mechanical Power and Capture Width}

In this section, the mechanical power output is computed by using the numerical model in OrcaFlex validated by experimental testing. The lines holding the SWEDE to the WindFloat are modeled as a power take-off system by adding mechanical damping in the simulation. The average mechanical energy $P_{\text {me }}$ received by one line is computed using the following equation:

$$
P_{m e}=\frac{1}{T} \int_{0}^{T} p(t) d t=\frac{1}{T} \int_{0}^{T} F(t) \cdot v(t) d t
$$

Where $p$ is the instantaneous power, $F$ is the line tension at the time $t$ and, and $v$ is the line velocity at the time $t$. $F$ and $v$ are both output at the three line ends connected to the SWEDE. Figure 9 presents an example of power output times-series for the sum of the three lines. 


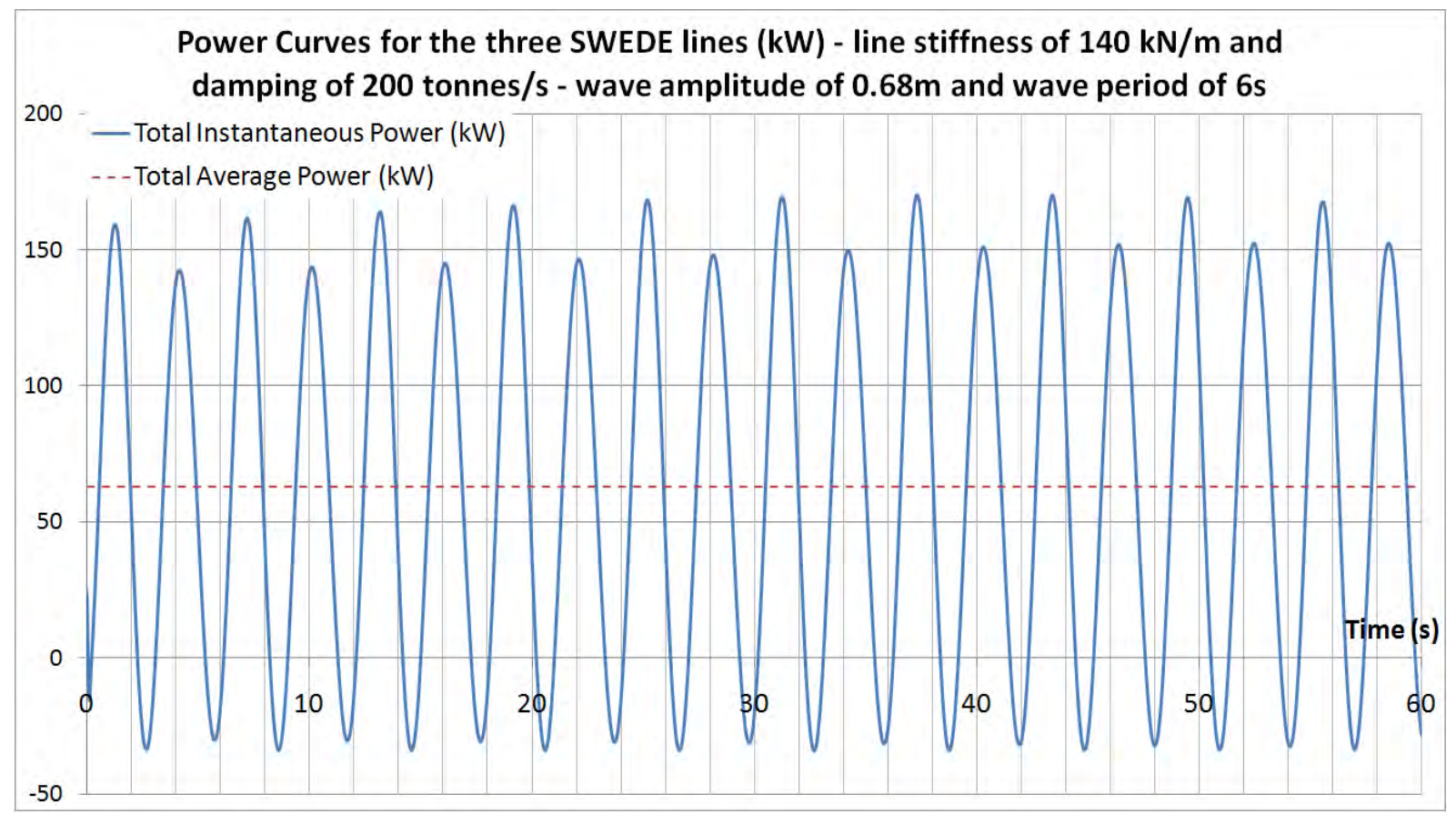

Figure 9: Example of time-series of power output for the three lines.

The normalized capture width CW of the SWEDE, representing the ratio of the width of the wave crest that is actually harnessed and the SWEDE diameter, is computed as [10]:

$$
C_{w}=\frac{1}{D} \frac{P_{m e}}{\frac{1}{2} \rho g A^{2} V_{g}}=\frac{1}{D} \frac{P_{m e}}{\frac{1}{8 \pi} \rho g^{2} A^{2} T}
$$

Where $\mathrm{D}$ is the diameter of the SWEDE, $\rho$ is the density of sea water, $\mathrm{A}$ is the wave amplitude, T is the wave period, and $V_{g}$ is the wave group velocity

Figure 10 presents the normalized capture width of the sphere for a constant line stiffness coefficient of $27 \mathrm{kN} / \mathrm{m}$, and a varying line damping coefficient. A capture width peak of 0.3 times the diameter of the SWEDE appears at $6 \mathrm{~s}$ for a damping coefficient of 500 tonnes/s. It means that for a regular wave of $6 \mathrm{~s}$, and the wave amplitude corresponding to the given wave slope, the wave power contained in a crest long of $3 \mathrm{~m}$ ( 0.3 times $10 \mathrm{~m}$, the SWEDE diameter) is completely converted into mechanical power. The maximum average mechanical power, maximum combination of force and velocity (the derivative of displacement), occurs at $6 \mathrm{~s}$ for two reasons. First, as shown on Figure 8, the tension in the SWEDE lines reaches a maximum at this period. Second, the surge/pitch motion of the sphere at a period of $6 \mathrm{~s}$ is relatively high, as shown on Figure 7 . The second peak in average mechanical power at a period of $4 \mathrm{~s}$ is due to the heave resonance of the SWEDE.

Figure 11 presents the normalized capture width of the sphere for a constant line stiffness coefficient of $140 \mathrm{kN} / \mathrm{m}$, and a varying line damping coefficient. There, a capture width peak of 0.6 times the diameter of the SWEDE appears at a wave period of $6 \mathrm{~s}$ for a damping coefficient of 200 tonnes/s. 


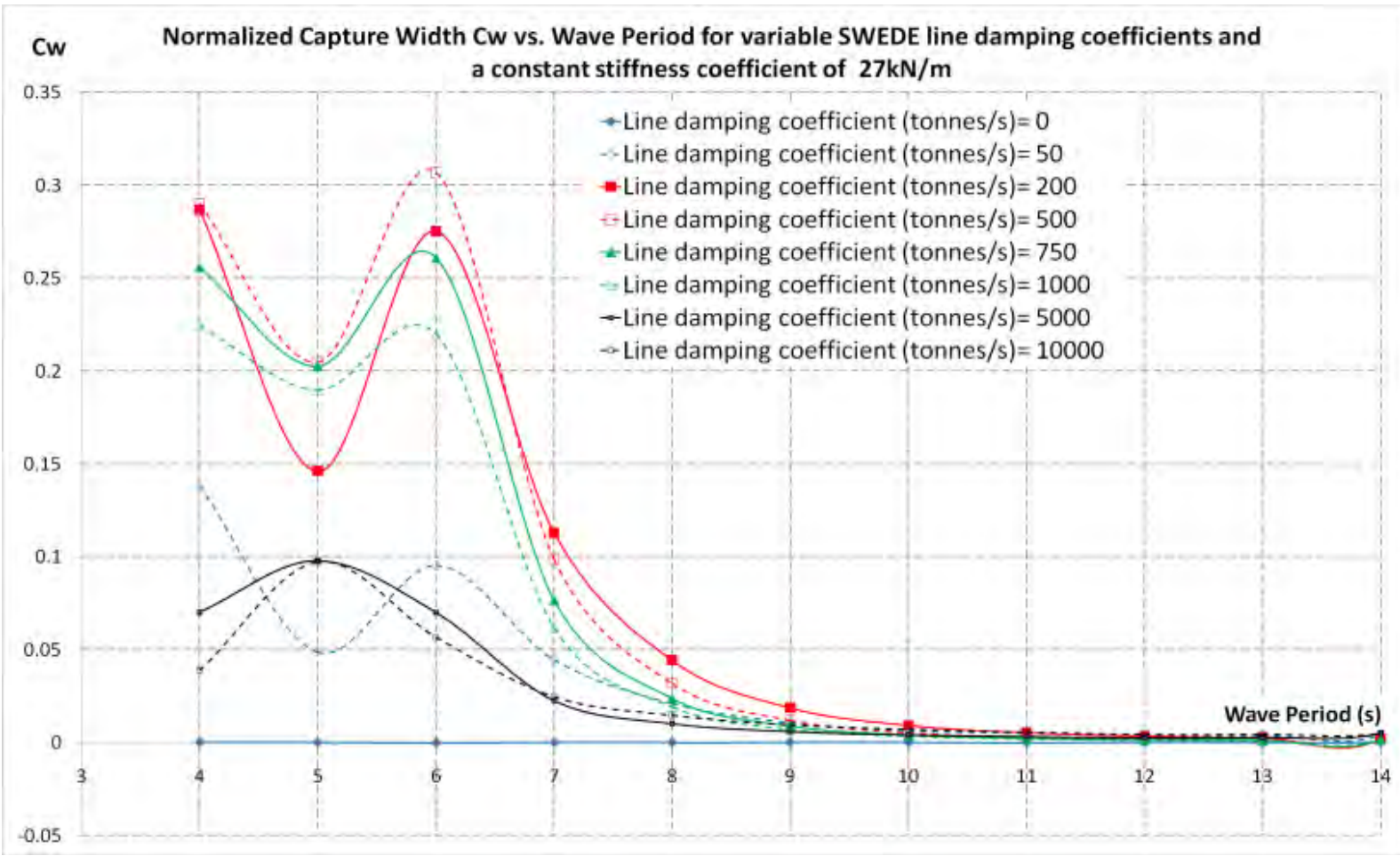

Figure 10: Normalized capture width for different SWEDE line damping coefficients and a stiffness coefficient of $27 \mathrm{kN} / \mathrm{m}$. The wave slope $W s$ is $12 \%$.

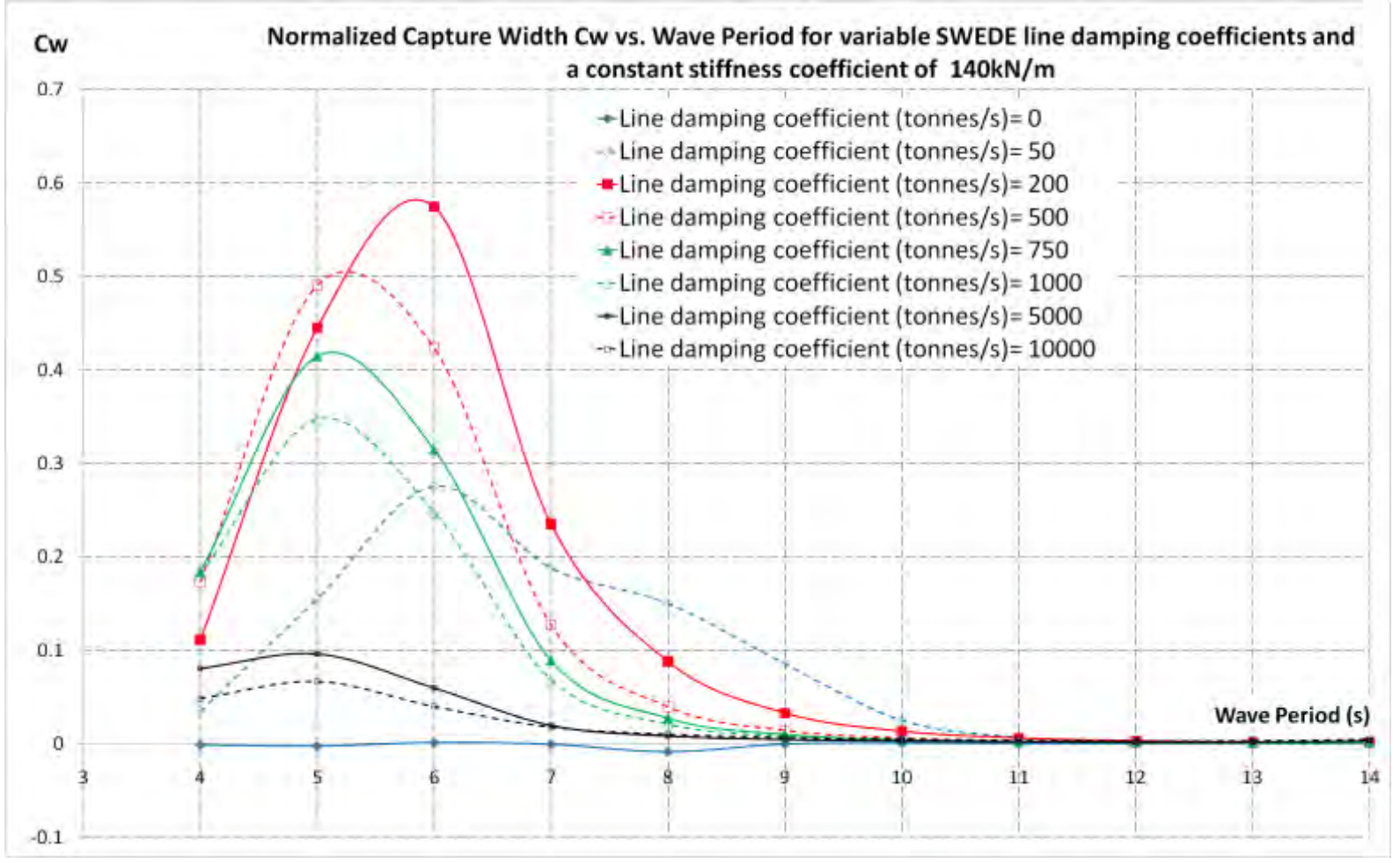

Figure 11: Normalized capture width for different SWEDE line damping coefficients and a stiffness coefficient of $140 \mathrm{kN} / \mathrm{m}$. The wave slope $W s$ is $12 \%$. 


\section{PTO (line) characteristics and wave period for maximum power extraction}

The stiffness and damping coefficients are varied for different wave periods (at a given wave slope, thus at a given wave amplitude) to spot the optimal wave power extraction point, which corresponds to the wave and PTO characteristics generating as much mechanical power as possible and as efficiently as possible, thus which corresponds to the maximal capture width. After a couple of trial and errors, this optimal point is discovered and occurs for a wave of $6 \mathrm{~s}$, a stiffness coefficient of $250 \mathrm{kN} / \mathrm{s}$ and damping coefficient of 50 tonnes/s. For example, about $389 \mathrm{~kW}$ of average mechanical power would be harnessed with a regular wave height of $2 \mathrm{~m}$, which corresponds to a normalized capture width of 1.65.

Table 2: Optimal operating point in regular waves

\begin{tabular}{|c|c|c|c|}
\hline & $\begin{array}{c}\text { Line Stiffness } \\
\text { coefficient } \\
(\mathbf{k N} / \mathrm{m})\end{array}$ & $\begin{array}{c}\text { Line Damping } \\
\text { coefficient (te/s) }\end{array}$ & $\begin{array}{c}\text { Maximum } \\
\text { Capture } \\
\text { Width }\end{array}$ \\
\hline $\begin{array}{c}\text { Maximum Power Output for one } \\
\text { wave period }\end{array}$ & 250 & 50 & 2.28 at T=6s \\
\hline
\end{tabular}

\section{Power/Capture Width matrices for different wave heights and periods in regular waves}

Two power and capture width matrices are derived using the PTO characteristics presented in Table 3.

Table 3: WWF SWEDE- Power matrix (different regular waves) at optimal operating point

\begin{tabular}{|c|c|c|c|c|c|c|c|c|}
\hline $\begin{array}{c}\text { Average } \\
\text { Power } \\
\text { (kW) }\end{array}$ & \multicolumn{8}{|c|}{ Wave Height $\mathrm{H}(\mathrm{m})$} \\
\hline$T(s)$ & 1 & 2 & 3 & 4 & 5 & 6 & 7 & 8 \\
\hline 4 & 4 & 16 & 36 & 66 & 107 & 157 & 214 & 276 \\
\hline 5 & 34 & 130 & 278 & 470 & 701 & 1,002 & 1,590 & 1,586 \\
\hline 6 & 134 & 389 & 695 & 1,044 & 1,436 & 1,862 & 2,354 & 2,903 \\
\hline 7 & 19 & 71 & 147 & 244 & 357 & 484 & 624 & 778 \\
\hline 8 & 2 & 8 & 19 & 33 & 52 & 75 & 103 & 137 \\
\hline 9 & 0 & 2 & 4 & 6 & 10 & 15 & 21 & 29 \\
\hline 10 & 0 & 0 & 1 & 2 & 3 & 4 & 6 & 8 \\
\hline 11 & 0 & 0 & 0 & 1 & 1 & 1 & 2 & 3 \\
\hline 12 & 0 & 0 & 0 & 0 & 0 & 1 & 1 & 2 \\
\hline 13 & 0 & 0 & 0 & 0 & 0 & 0 & 0 & 1 \\
\hline 14 & (0) & 0 & 0 & 0 & 0 & 0 & 0 & - \\
\hline
\end{tabular}




\begin{tabular}{|c|c|c|c|c|c|c|c|c|}
\hline \multirow{2}{*}{$\begin{array}{c}\text { Capture } \\
\text { Width } \\
\text { Tp (s) }\end{array}$} & \multicolumn{8}{|c|}{ Wave Height $\mathbf{H}(\mathrm{m})$} \\
\hline & 1.0 & 2.0 & 3.0 & 4.0 & 5.0 & 6.0 & 7.0 & 8.0 \\
\hline 4 & 0.10 & 0.10 & 0.10 & 0.11 & 0.11 & 0.11 & 0.11 & 0.11 \\
\hline 5 & 0.69 & 0.66 & 0.63 & 0.60 & 0.57 & 0.57 & 0.66 & 0.50 \\
\hline 6 & 2.28 & 1.65 & 1.31 & 1.11 & 0.98 & 0.88 & 0.82 & 0.77 \\
\hline 7 & 0.28 & 0.26 & 0.24 & 0.22 & 0.21 & 0.20 & 0.19 & 0.18 \\
\hline 8 & 0.03 & 0.03 & 0.03 & 0.03 & 0.03 & 0.03 & 0.03 & 0.03 \\
\hline 9 & 0.00 & 0.00 & 0.00 & 0.00 & 0.00 & 0.00 & 0.00 & 0.01 \\
\hline 10 & 0.00 & 0.00 & 0.00 & 0.00 & 0.00 & 0.00 & 0.00 & 0.00 \\
\hline 11 & 0.00 & 0.00 & 0.00 & 0.00 & 0.00 & 0.00 & 0.00 & 0.00 \\
\hline 12 & 0.00 & 0.00 & 0.00 & 0.00 & 0.00 & 0.00 & 0.00 & 0.00 \\
\hline 13 & 0.00 & 0.00 & 0.00 & 0.00 & 0.00 & 0.00 & 0.00 & 0.00 \\
\hline 14 & $(0.00)$ & 0.00 & 0.00 & 0.00 & 0.00 & 0.00 & 0.00 & - \\
\hline
\end{tabular}




\subsection{Oscillating Wave Surge Converter (OWSC or FLAPS)}

\subsubsection{Validation of the numerical models}

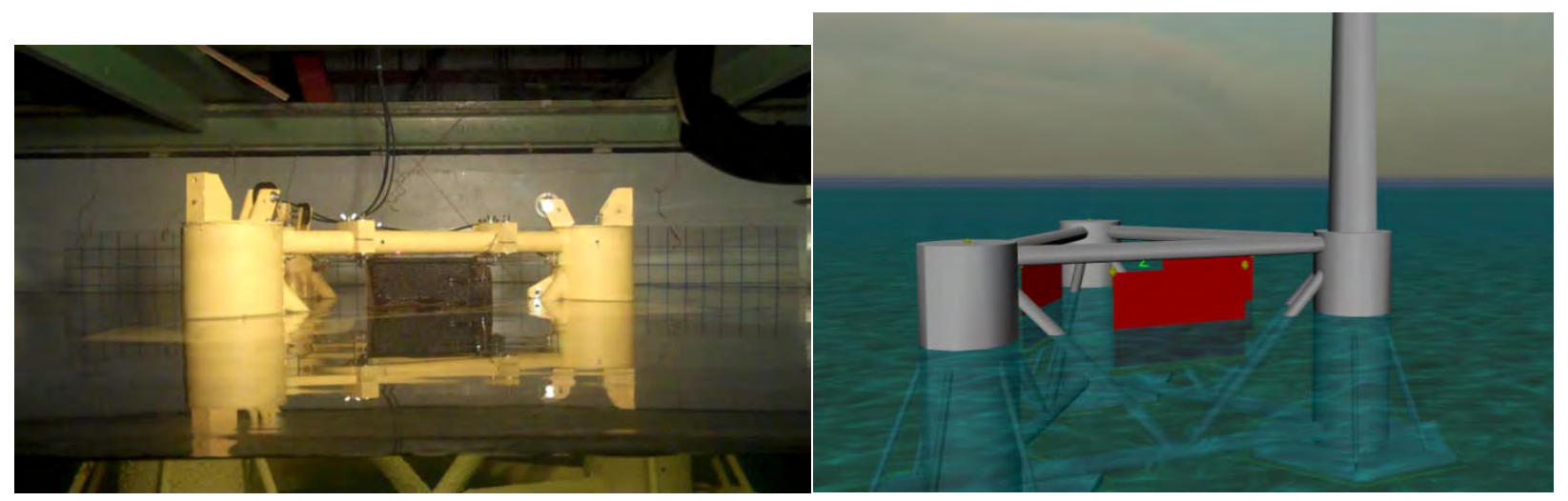

Figure 12: Picture of the WindWaveFloat-FLAPS model in the UC Berkeley towing tank and side view of OrcaFlex WindWaveFloat numerical model incorporating the FLAPS.

The incoming waves, by hitting the FLAPS, induce a swinging pitch motion. In OrcaFlex, the lines (spring and damper) are attached to the bottom of the flap in a parallel configuration. During the experimental tests, a torsion spring is used with the rotational stiffness corresponding to the linear stiffness of the springs attached to the bottom of the flaps in OrcaFlex.

The WindFloat platform numerical model validation is described in the $2^{\text {nd }}$ quarter report. Only the FLAPS numerical models and the WindWaveFloat numerical models are discussed in this section. The FLAPS damping coefficients and drag coefficients in pitch are adjusted in the OrcaFlex WindWaveFloat numerical model to match the time-series of FLAPS motions on the WindWaveFloat model at 1:78.5 performed during the tank tests.

The FLAPS pitch natural period is given in Table 4 for the FLAP line stiffness coefficient tested in the wave basin ( $80 \mathrm{kN} / \mathrm{m}$ at full scale).

Table 4: FLAPS natural period (full scale)

\begin{tabular}{|l|c|}
\cline { 2 - 2 } \multicolumn{1}{c|}{} & $\begin{array}{c}\text { Flap line stiffness } \\
\text { coefficient of } 80 \mathrm{kN} / \mathrm{m}\end{array}$ \\
\hline FLAPS Pitch natural period (s) & 7.0 \\
\hline
\end{tabular}

Two types of results are then presented. First, the experimental RAOs of the WindFloat alone are compared with the experimental RAOs of the WindWaveFloat with the FLAPS to study the impact of the FLAPS on the WindFloat platform. Second, the theoretical and experimental pitch RAOs of the FLAPS are presented. No damping is added to the lines at this point, because the aim is to validate the numerical models with the experimental tests, and no damping was used in the first series of experimental tests reported here. 


\section{WindFloat RAOs Comparisons: Impact of the FLAPS on the WindFloat platform}

To understand the impact of the FLAPS on the WindFloat motions, the RAOs of the WindFloat alone and the RAOs of the WindWaveFloat with the FLAPS, both measured experimentally, are compared for two wave slopes on Figure 13, for a line stiffness coefficient of $80 \mathrm{kN} / \mathrm{m}$. The wave slope $W s$ is defined as the ratio of the wave amplitude and the wavelength. In this section, a small wave slope refers to $6 \%$, and a large wave slope refers to $12 \%$.

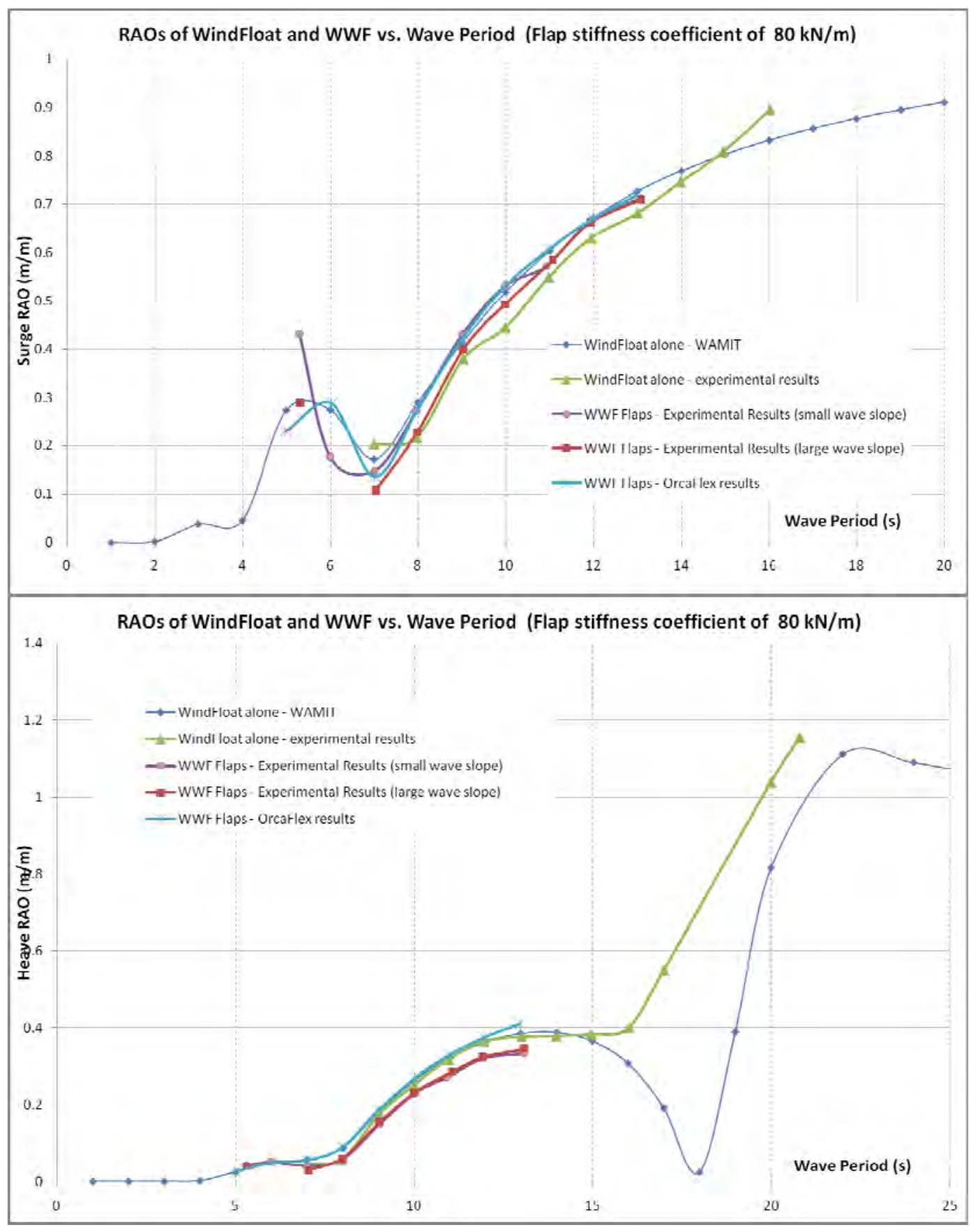




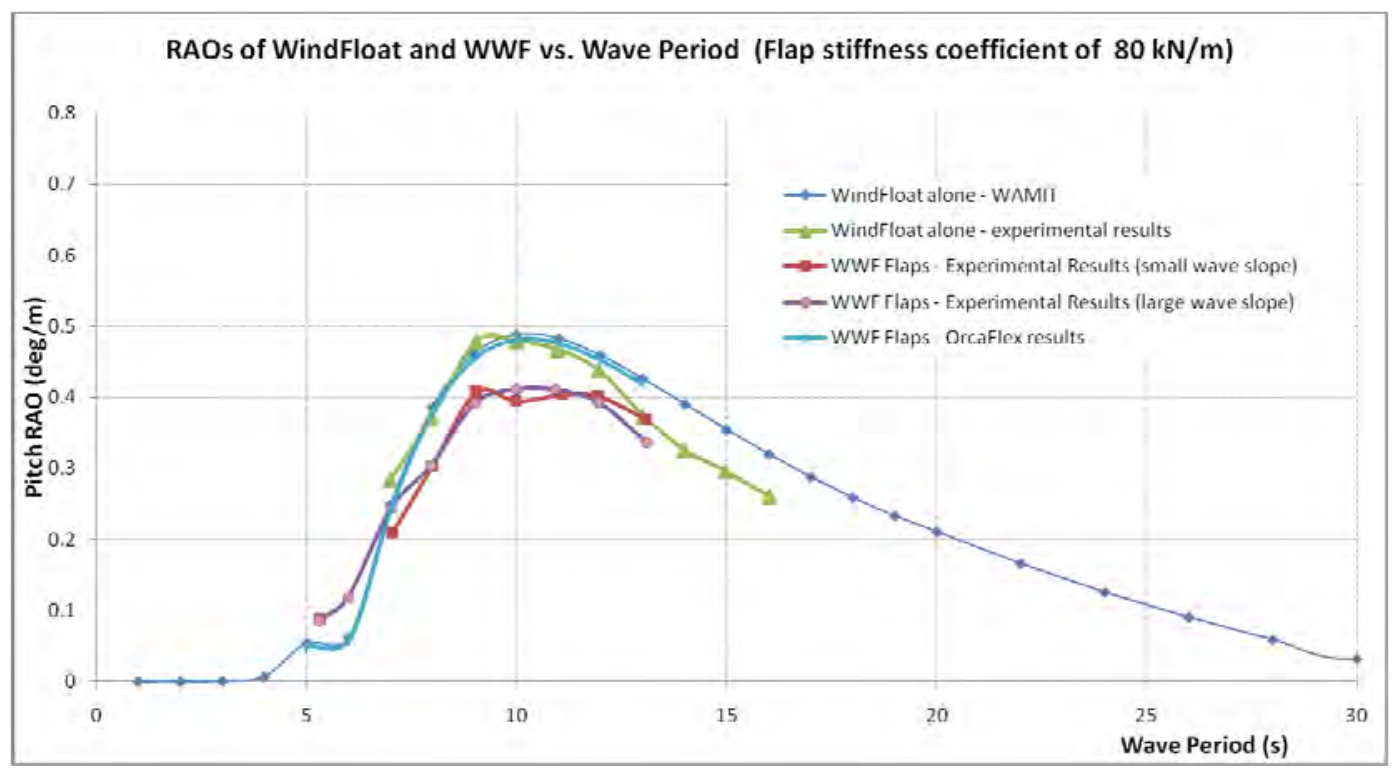

Figure 13: Comparison of the Surge, Heave, and Pitch RAOs of the WWF and the WindFloat alone. The wave slope Ws is $6 \%$.

It can be seen that the FLAPS minimally affects the motions of the WindFloat platform. The FLAPS have no impact on the WindFloat heave and surge motion. However, one can notice a slight rise of WindFloat pitch RAO around the FLAPS pitch natural period, then a slight decline of WindFloat pitch RAO after the FLAPS pitch natural period.

The limitation in this comparison of WindFloat RAOs vs. WindWaveFloat RAOs is that results are presented only for given FLAPS line stiffness coefficients and a zero damping coefficient. Future numerical simulations must be performed to make sure that the same conclusions can be drawn for any FLAPS line stiffness and damping coefficients, especially combinations that convert a significant amount of wave power into mechanical power.

\section{FLAPS Motion RAOS}

The numerical and experimental FLAPS motion RAOs are presented in Figure 14 for flap \#1, which is the flap facing the $0^{\circ}$ - incoming waves directly (hinged between WindFloat column 2 and 3). On Figure 15, the Pitch RAOs of the three flaps are presented for a large wave slope. There is no damping in these simulations to verify the extreme motions.

The FLAPS pitch natural period in pitch of $7 \mathrm{~s}$ is retrieved for this line stiffness coefficient. The match between numerical and experimental results is what is really sought for, and confirmed here. A slight difference in peak amplitude is observed for a larger wave slope, probably due to a slight miscalculation of hydrodynamic damping for a larger wave slope. 


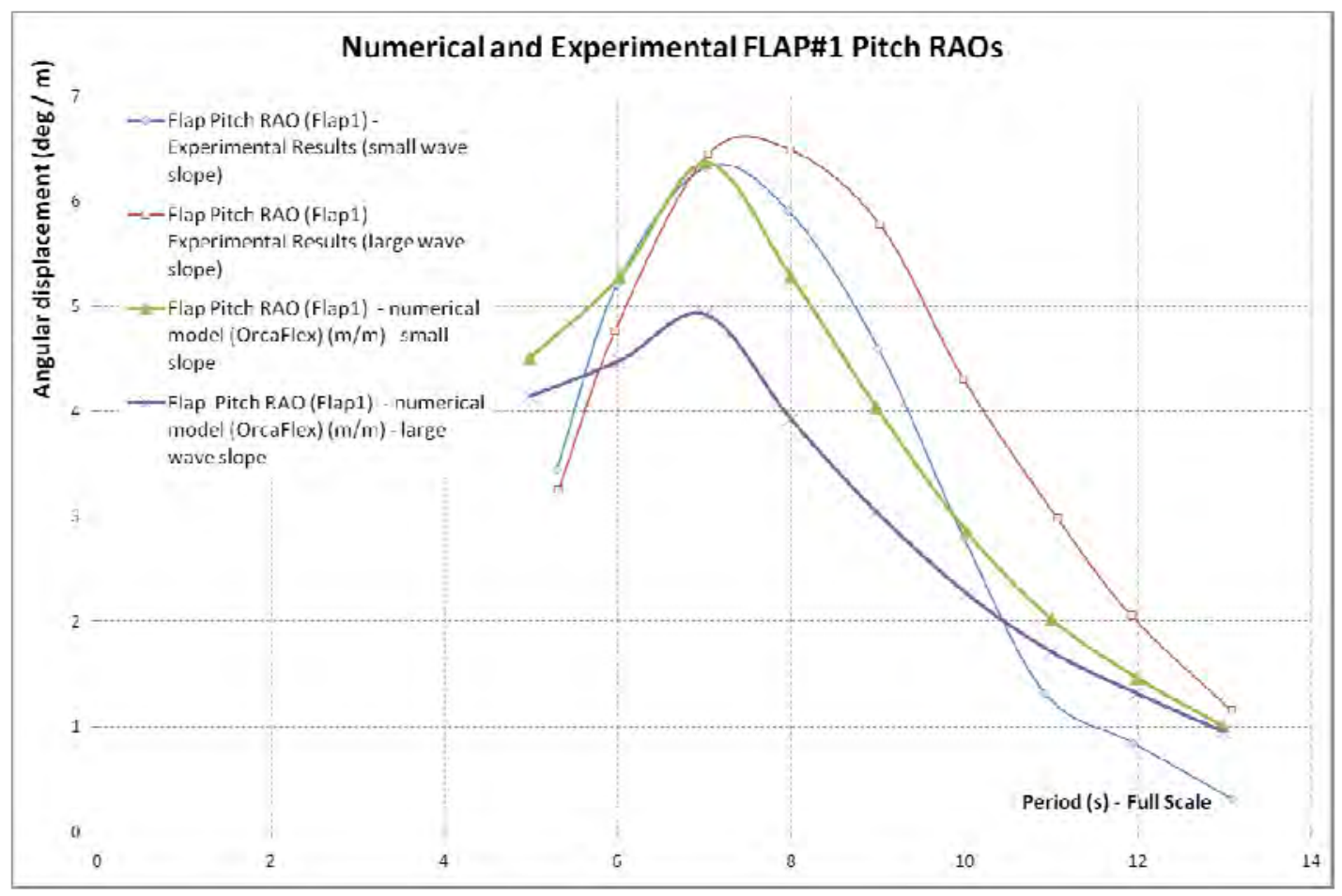

Figure 14: Comparison of numerical vs. experimental FLAP\#1 Pitch RAO on the WindWaveFloat for a line stiffness coefficient of $80 \mathrm{kN} / \mathrm{m}$.

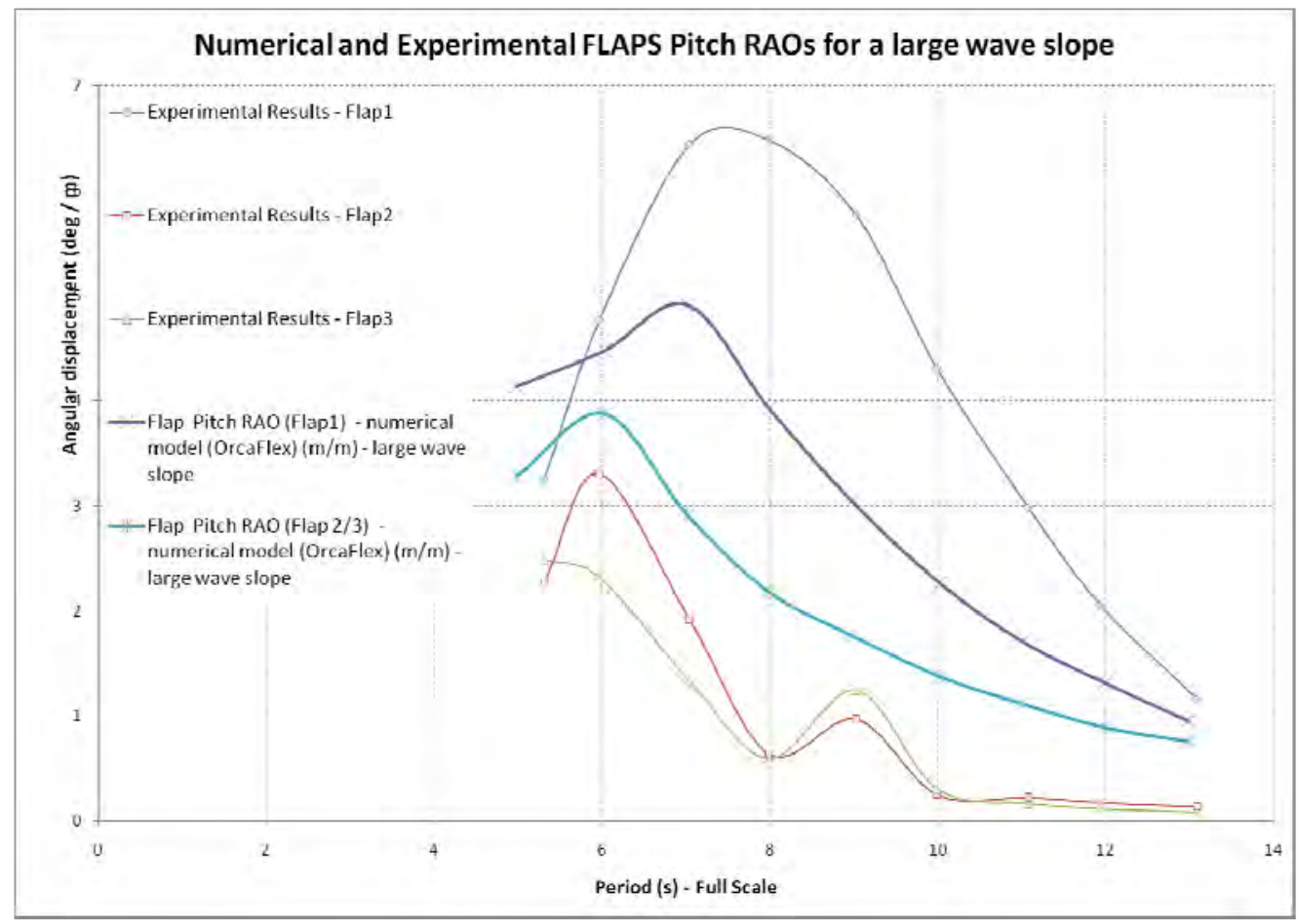

Figure 15: Comparison of numerical vs. experimental FLAPS RAOs on the WindWaveFloat for a line stiffness coefficient of $140 \mathrm{kN} / \mathrm{m}$. 
This gives confidence in the power prediction that will be obtained numerically as it is calculated from the product of the force by the rate of change of motion. The match between experiments and theory is strong for a small wave slope.

\subsubsection{Power Prediction}

Now that the numerical models are validated, some damping is added in the lines holding the FLAPS, to simulate the presence of a power take-off system. The average mechanical power generated by the FLAPS and the capture width are thus computed for different damping coefficients at the two tested line stiffness coefficients.

\section{Mechanical Power and Capture Width}

The mechanical power output is computed by using the numerical model in OrcaFlex validated by experimental testing. The lines holding the FLAPS to the WindFloat are modeled as a power take-off system by adding mechanical damping in the simulation. The average mechanical energy $P_{m e}$ received by one line is computed using the following equation:

$$
P_{m e}=\frac{1}{T} \int_{0}^{T} p(t) d t=\frac{1}{T} \int_{0}^{T} F(t) \cdot v(t) d t
$$

The total average power is the sum of the average power of the six lines holding the three flaps.

The normalized capture width CW of the FLAPS, representing the ratio of the width of the wave crest that is actually harnessed and the FLAPS width, is computed as [10]:

$$
C_{w}=\frac{1}{D} \frac{P_{m e}}{\frac{1}{2} \rho g A^{2} V_{g}}=\frac{1}{D} \frac{P_{m e}}{\frac{1}{8 \pi} \rho g^{2} A^{2} T}
$$

Where $D$ is the width of the FLAPS $(D=16 m), \rho$ is the density of sea water, $A$ is the wave amplitude, $T$ is the wave period, and $V_{g}$ is the wave group velocity

\section{PTO (line) characteristics and wave period for maximum power extraction}

The stiffness and damping coefficients are varied for different wave periods (at a given wave slope, thus at a given wave amplitude) to spot the optimal wave power extraction point, which corresponds to the wave and PTO characteristics generating as much mechanical power as possible and as efficiently as possible, thus which corresponds to the maximal capture width. After a couple of trial and errors, this optimal point is discovered and occurs for a wave of $5 \mathrm{~s}$, a stiffness coefficient of $200 \mathrm{kN} / \mathrm{s}$ and damping coefficient of 200 tonnes/s (because the FLAPS pitch natural period is down to $5 \mathrm{~s}$ in that case). For example, about $139 \mathrm{~kW}$ of average mechanical power would be harnessed with a regular wave height of $2 \mathrm{~m}$, which corresponds to a normalized capture width of 0.44 . 
Table 5: Optimal operating point in regular waves

\begin{tabular}{|c|c|c|c|}
\hline & $\begin{array}{c}\text { Line Stiffness } \\
\text { coefficient } \\
(\mathbf{k N} / \mathrm{m})\end{array}$ & $\begin{array}{c}\text { Line Damping } \\
\text { coefficient (te/s) }\end{array}$ & $\begin{array}{c}\text { Maximum } \\
\text { Capture } \\
\text { Width }\end{array}$ \\
\hline $\begin{array}{c}\text { Maximum Power Output for one } \\
\text { wave period }\end{array}$ & 200 & 200 & 0.57 at $\mathrm{T}=5 \mathrm{~s}$ \\
\hline
\end{tabular}

Power/Capture Width matrices for different wave heights and periods in regular waves

Two power and capture width matrices are derived using the PTO characteristics presented in Table 6.

Table 6: WWF FLAPS - Power matrix (different regular waves) at optimal operating point

\begin{tabular}{|c|c|c|c|c|c|c|c|c|}
\hline $\begin{array}{c}\text { Average } \\
\text { Power } \\
(\mathbf{k W})\end{array}$ & \multicolumn{8}{|c|}{ Wave Height $\mathbf{H}(\mathrm{m})$} \\
\hline $\operatorname{Tp}(s)$ & 1 & 2 & 3 & 4 & 5 & 6 & 7 & 8 \\
\hline 4 & 36 & 126 & 257 & 423 & 615 & 832 & 1,073 & 1,344 \\
\hline 5 & 45 & 139 & 260 & 398 & 551 & 716 & 892 & 1,078 \\
\hline 6 & 29 & 84 & 157 & 250 & 365 & 504 & 669 & 862 \\
\hline 7 & 12 & 38 & 77 & 131 & 203 & 295 & 409 & 545 \\
\hline 8 & 5 & 16 & 35 & 64 & 103 & 155 & 220 & 299 \\
\hline 9 & 2 & 7 & 16 & 31 & 51 & 80 & 116 & 160 \\
\hline 10 & 1 & 3 & 7 & 14 & 25 & 39 & 58 & 82 \\
\hline 11 & 0 & 1 & 3 & 7 & 12 & 19 & 29 & 42 \\
\hline 12 & 0 & 1 & 2 & 3 & 6 & 10 & 15 & 22 \\
\hline 13 & 0 & 0 & 1 & 2 & 3 & 5 & 7 & 11 \\
\hline 14 & 0 & 0 & 0 & 1 & 1 & 2 & 4 & 5 \\
\hline
\end{tabular}

\begin{tabular}{|c|c|c|c|c|c|c|c|c|}
\hline $\begin{array}{l}\text { Capture } \\
\text { Width }\end{array}$ & \multicolumn{8}{|c|}{ Wave Height $\mathrm{H}$ (m) } \\
\hline $\mathrm{Tp}(\mathrm{s})$ & 1.0 & 2.0 & 3.0 & 4.0 & 5.0 & 6.0 & 7.0 & 8.0 \\
\hline 4 & 0.57 & 0.50 & 0.46 & 0.42 & 0.39 & 0.37 & 0.35 & 0.33 \\
\hline 5 & 0.57 & 0.44 & 0.37 & 0.32 & 0.28 & 0.25 & 0.23 & 0.21 \\
\hline 6 & 0.30 & 0.22 & 0.19 & 0.17 & 0.15 & 0.15 & 0.15 & 0.14 \\
\hline 7 & 0.11 & 0.09 & 0.08 & 0.07 & 0.07 & 0.07 & 0.08 & 0.08 \\
\hline 8 & 0.04 & 0.03 & 0.03 & 0.03 & 0.03 & 0.03 & 0.04 & 0.04 \\
\hline 9 & 0.01 & 0.01 & 0.01 & 0.01 & 0.01 & 0.02 & 0.02 & 0.02 \\
\hline 10 & 0.00 & 0.00 & 0.01 & 0.01 & 0.01 & 0.01 & 0.01 & 0.01 \\
\hline
\end{tabular}




\begin{tabular}{|l|llllllll|}
11 & 0.00 & 0.00 & 0.00 & 0.00 & 0.00 & 0.00 & 0.00 & 0.00 \\
\hline 12 & 0.00 & 0.00 & 0.00 & 0.00 & 0.00 & 0.00 & 0.00 & 0.00 \\
\cline { 1 - 1 } & 0.00 & 0.00 & 0.00 & 0.00 & 0.00 & 0.00 & 0.00 & 0.00 \\
\cline { 1 - 1 } & 0.00 & 0.00 & 0.00 & 0.00 & 0.00 & 0.00 & 0.00 & 0.00 \\
\hline
\end{tabular}




\subsection{Oscillating Water Column (OWC)}

\subsubsection{Validation of the numerical models}

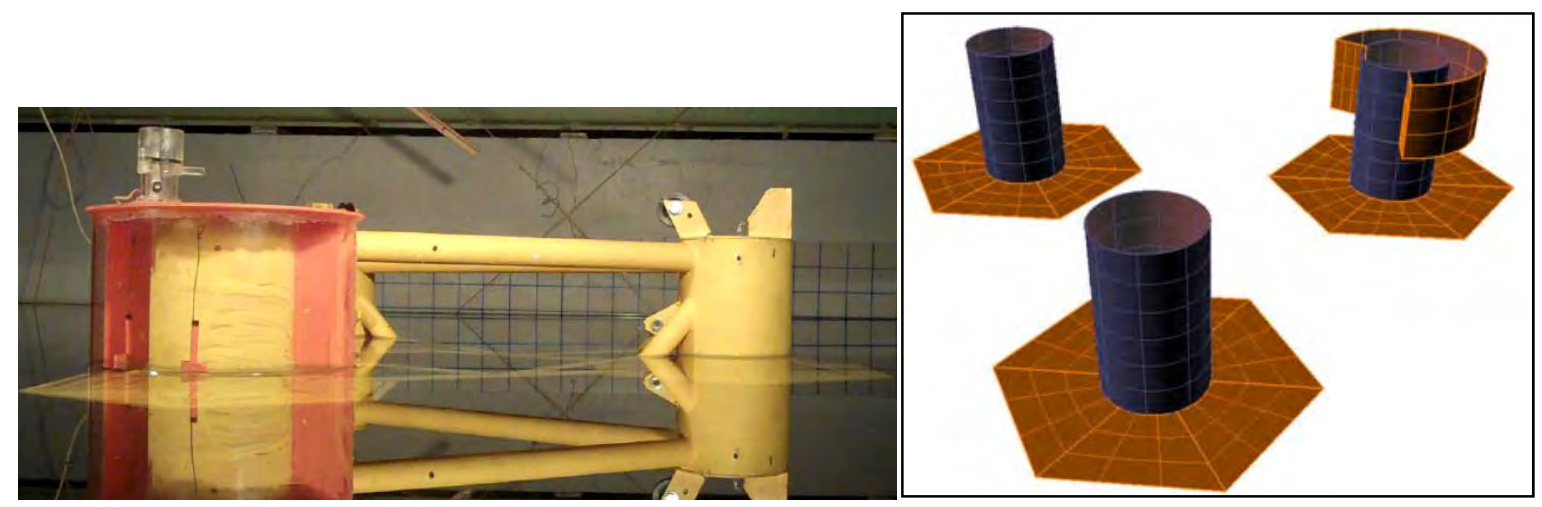

Figure 16 a, b: Picture of the WindWaveFloat-OWC model in the UC Berkeley towing tank and B-Spline Representation of WindWaveFloat Submerged Areas in Rhino3D software

A more complete description of the numerical modeling of the system, summarized here, can be found in the $2^{\text {nd }}$ quarter report. A numerical model of the WindWaveFloat with OWC is built in the frequency domain, by using linear diffraction-radiation software WAMIT with generalized modes. The displacements and rotations of the WindFloat represent the first six degrees-of-freedom. The internal free surface motions are calculated using two generalized modes; the first generalized mode corresponds to the pumping mode, and the second generalized mode corresponds to the slope of the sloshing mode. The pumping mode is defined relatively to the vertical rigid-motion of the platform. The power take-off system (called PTO), here a Wells turbine, is mimicked by an additional equivalent stiffness coefficient $\left(\mathrm{K}_{\mathrm{pto}}\right)$ and damping coefficient $\left(\mathrm{B}_{\mathrm{pto}}\right)$ on the pumping motion of the free surface relative to the platform body.

The system of motion equations for the 8-DoF WindWaveFloat device may be written, in the frequency domain, by:

$$
\left[-\omega^{2}(M+A)+\left(C+K_{p t o}\right)\right] \hat{x}+i \omega\left(B+B_{p t o}\right) \hat{x}=\hat{F}
$$

where $\mathrm{x}$ is the 8-DoF displacement vector including internal free surface sloshing and pumping, $\omega$ the angular frequency, $M$ the $8 \times 8$ mass matrix of the WindWaveFloat-OWC, $A$ the $8 \times 8$ frequency-dependent added mass matrix, $B$ the $8 \times 8$ frequency-dependent damping matrix, $C$ the $8 \times 8$ stiffness matrix, and $F$ the incident wave excitation force. The hat symbol, ^ , denotes complex amplitude. From this equation, the complex amplitudes of the platform and the free surface displacements can be computed.

Two approaches have been undertaken when calculating the RAOs.

- $\quad$ Using WAMIT directly, a constant linear damping is applied to all frequencies.

- Using a post processing code where the equations of motion, in the frequency domain, are expressed as functions of the complex amplitudes of the displacements. Thus, by means of the 
hydrodynamic coefficients (added mass and damping) and the complex amplitudes of the excitation loads, the RAOs are determined for particular PTO characterizations, i.e. for specified external damping. This way the PTO damper stiffness may be adjusted to each frequency in order to get a prompt idea about the power absorption under different conditions. This second method enables to optimize the PTO damping to maximize the power take-off.

Model tests are performed to validate the numerical results and calibrate the numerical model with appropriate damping values, usually underestimated by WAMIT. A model of 1:78 scale is built in acrylic. The platform is attached to an equivalent mooring to match the global stiffness in surge, sway and yaw. To assess the effect of global radiation on the internal free surface elevation, the model can be fixed as well. Regular waves are generated with steepness between $1 / 80$ and $1 / 30$.

To estimate the performance of the OWC, the flow rate exiting the chamber and the pressure drop between the chamber and the outside environment are measured. The turbine is modeled with a 1 inch diameter opening at the top of the air chamber. This corresponds to a $2 \mathrm{~m}$ diameter turbine in full scale. The PTO damping is modeled with a thin porous carpet tightly fitted to obstruct this opening. This method of modelization of the turbine has been used in similar setups. It is an acceptable model of a Wells turbine, provided that a linear relation can be established between the air flow and the pressure drop through the carpet. The pressure drop is obtained by measuring the pressure inside the chamber, upstream of the carpet. The downstream pressure, outside the chamber, is equal to atmospheric pressure. This system is bi-directional, like a Wells turbine.

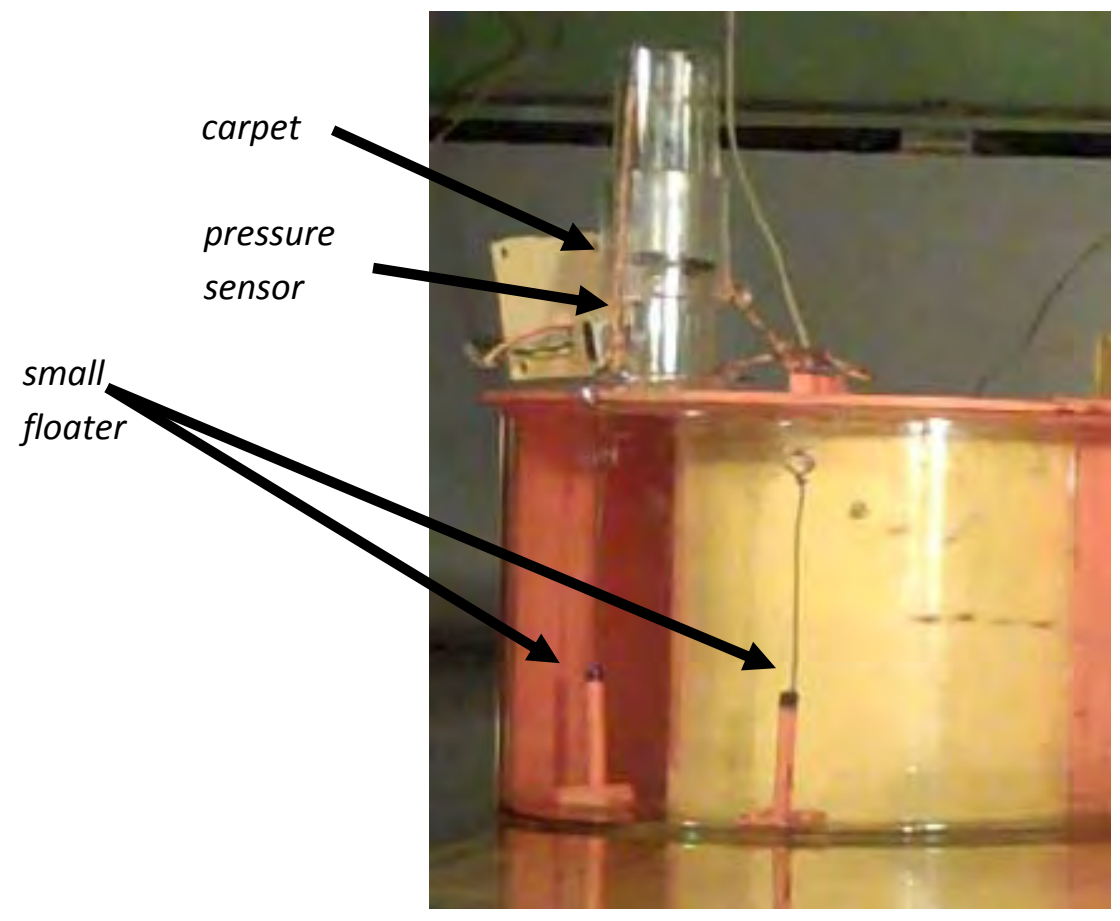

Figure 17: Pressure and Flow Measurements in the OWC of the WindWaveFloat 
Three types of results are then presented. First, the OWC chamber characteristics are validated by using an open chamber (no carpet, so no PTO damping). The pumping and sloshing modes are investigated in this section and numerical results are validated through experiments. Second, to experimentally simulate a Wells turbine, a permeable carpet is used to introduce a pressure drop proportional to the flow rate, thus mimicking the PTO damping. The validation of the power take-off modeling is presented in this section. Third, the experimental RAOs of the WindFloat alone are compared with the experimental RAOs of the WindWaveFloat with the OWC to study the impact of the OWC on the WindFloat platform.

\section{Experimental validation of OWC Chamber Characteristics}

A decay test is carried out during the model tests to verify the period of resonance of the internal free surface in the OWC air chamber. The measured natural period is $6.91 \mathrm{sec}$. The period of resonance of the pumping and sloshing modes can be estimated numerically. Provided that cross-flow effects are minimized, it is given by: $T_{i}=2 \pi \sqrt{\frac{A_{i, i}}{C_{i, i}}}$ (4)

Where $A_{i, i}$ is the added mass coefficient of the OWC chamber in mode $i$ and $C_{i, i}$ the stiffness coefficient of the chamber.

Using WAMIT calculations for the added mass and stiffness coefficient, the period of resonance of the pumping mode of the chamber is equal to $6.98 \mathrm{sec}$, as shown in Table 7.

Table 7: Dynamic Characteristics of OWC Chamber

\begin{tabular}{|l|c|r|r|}
\hline \multirow{2}{*}{} & \multirow{2}{*}{ Unit } & \multicolumn{2}{|c|}{ Internal Free Surface Mode } \\
\cline { 3 - 4 } & & Pumping & \multicolumn{1}{c|}{ Sloshing } \\
\hline Added Mass & kg & $1.45 \mathrm{E}+06$ & $3.40 \mathrm{E}+08$ \\
\hline Stiffness & kg.s-2 & $1.18 \mathrm{E}+06$ & $4.82 \mathrm{E}+08$ \\
\hline Period & $\mathrm{s}$ & 6.98 & 5.25 \\
\hline
\end{tabular}

Response Amplitude Operators (RAOs) of internal free surface motion are obtained experimentally, with the chamber open. The air flow can exit the chamber freely through the turbine orifice since no damping is applied. Experimental results are compared for two wave steepness to highlight non-linear damping effects. In the case of the steeper wave, internal free surface vertical elevation is compared when the WindFloat model is free floating and fixed. The resulting pumping and sloshing RAOs are plotted in Figure 18 and Figure 19 respectively. 


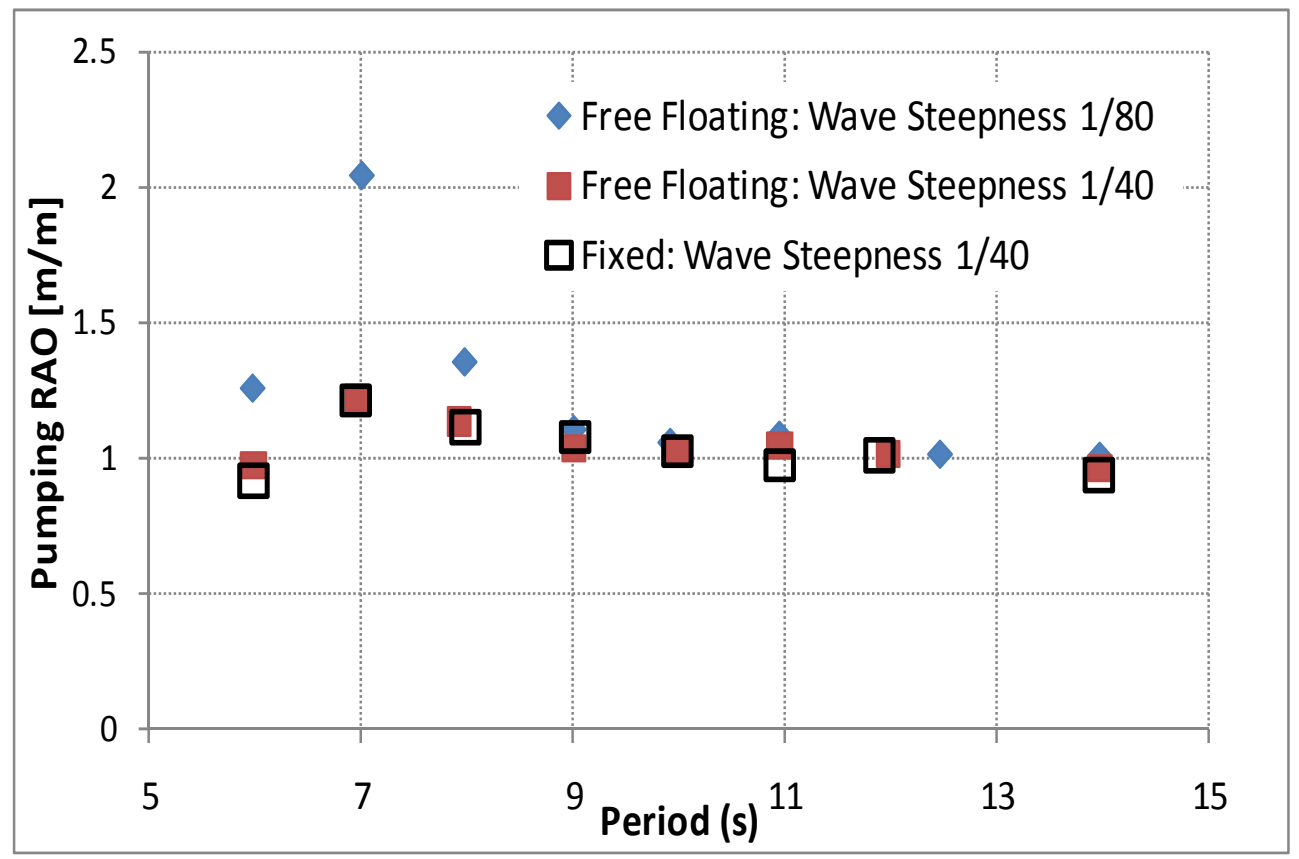

Figure 18: Amplitude of RAO of pumping mode in air chamber of OWC - Experimental results

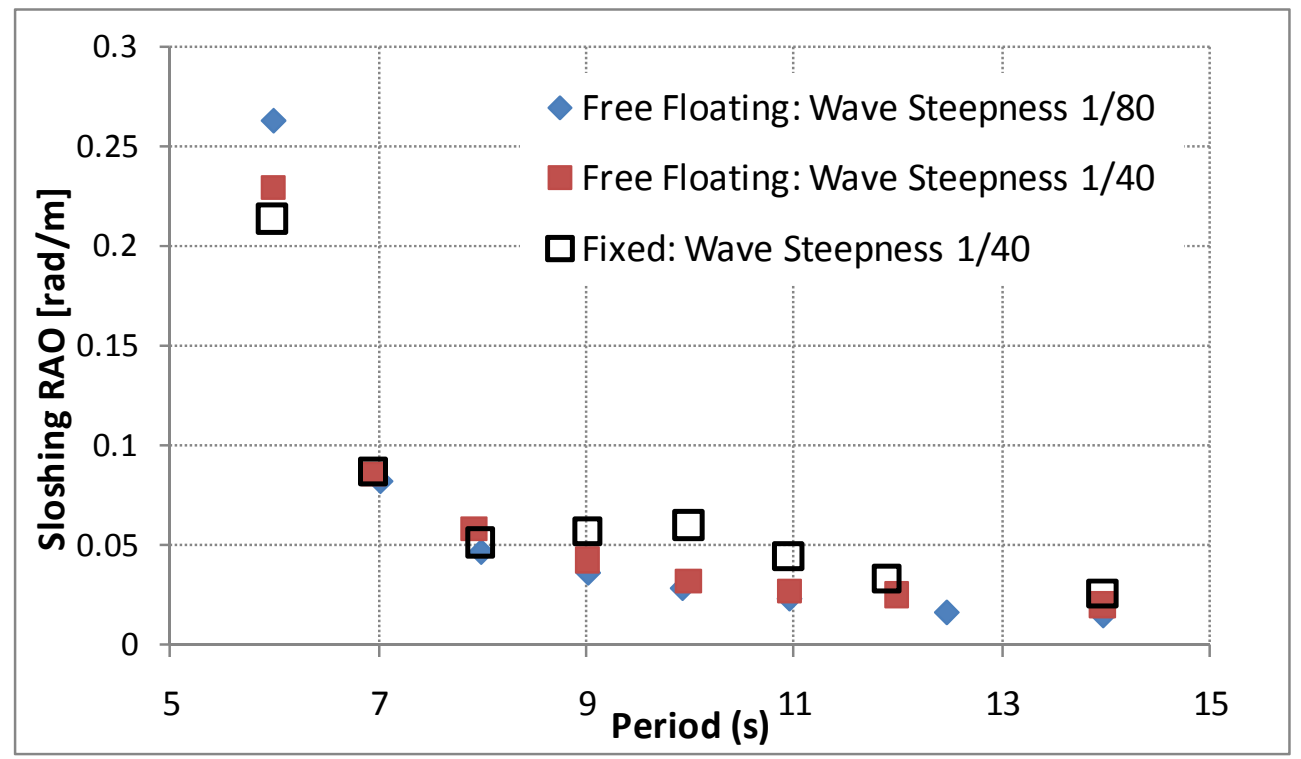

Figure 19: Amplitude of RAO of sloshing mode in air chamber of OWC - Experimental results 
The resonance of the internal free surface pumping mode is captured in Figure 18. The resonance of the sloshing mode is lower than 6 seconds as predicted. It was not captured due to the limitations of the model scale. Sloshing decreases rapidly when the period increases. It is small in the studied range of frequency, but it could affect the results at the period of resonance. Besides, as long as it remains small enough to avoid disturbance to the flow rate, it is not essential to the calculation of the output power. Results presented here focus on the pumping mode.

Results are not significantly different between the fixed case and the free floating case. At constant steepness, the radiation potential in the 6 global degrees of freedom doesn't affect the pumping mode. However, the amplitude of the resonance varies with the steepness of the wave, as observed in the floating case. This reflects the contribution of non-linear damping from viscous effects and air flow reaction in the chamber.

The pumping RAO is compared with numerical predictions in the floating case. When wave radiation damping only is included in the computation, the RAO amplitude is over-predicted. To quantify the amount of additional damping, numerical results are generated with an additional constant damping. It is defined as a fraction of critical damping, which in the case of pumping mode is:

$$
B_{77}=2 \sqrt{A_{77} C_{77}}
$$

Results in Figure 20 show that, at the resonance, an equivalent linear damping equal to $7 \%$ of critical damping is necessary to match the experimental results with wave steepness of $1 / 80$. With steeper waves, the equivalent damping increases to $12 \%$ of critical damping.

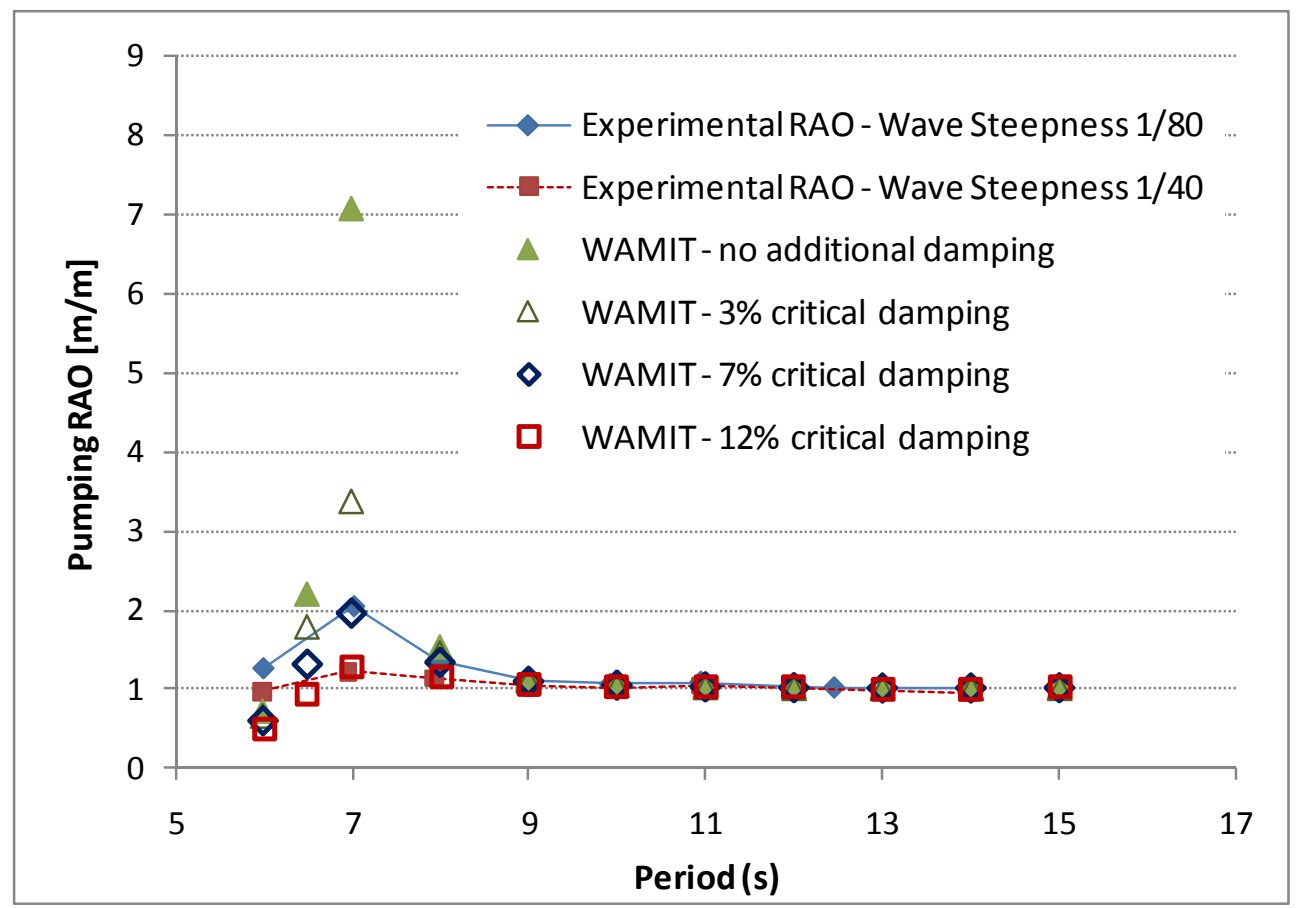

Figure 20: Comparison of WAMIT predictions and Experimental measurements of Internal Free Surface Pumping RAO in the free floating case $[\mathrm{m} / \mathrm{m}]$ 


\section{Modeling of the Power Take-off System}

The power take-off is modeled experimentally with a carpet. The linearity of the carpet is verified by measuring the instantaneous pressure drop inside the chamber and the flow rate, in regular waves. The RMS of the non-dimensional pressure $\Psi$ is plotted against the RMS of the flow rate coefficient $\Phi$ in Figure 21 for regular waves of constant steepness. As a reminder, $\Psi_{c}=K_{c} \Phi_{c}$, where the pressure coefficient $\Psi_{c}$ and flow coefficient $\Phi_{c}$ are:

$$
\psi_{c}=\frac{p}{\rho_{0} g H} \text { and } \Phi_{c}=\frac{2 \pi \dot{m}}{\rho_{0} \omega S H}
$$

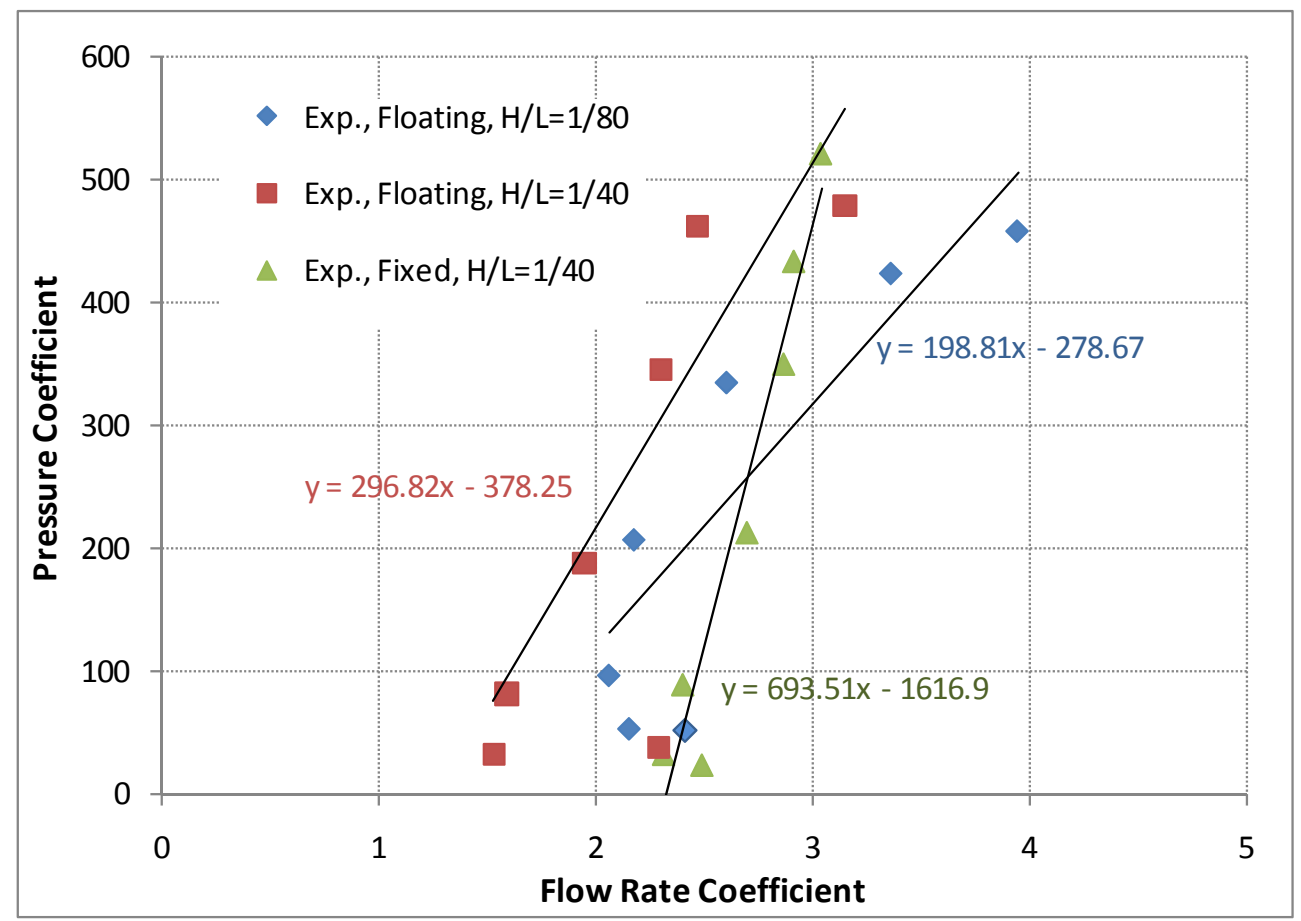

Figure 21: Experimental Relationship between Pressure Coefficient and Flow Rate Coefficient with varying Wave Steepness

Here, the fluid is assumed incompressible, so that the mass flow rate is proportional to the volumetric flow rate. To attempt to model the problem numerically, the relation between pressure and flow coefficients must be linearized. At constant steepness, the relation between the pressure coefficient and the flow rate coefficient are approximated by a linear regression. It is notable that this linear regression breaks down for small pressure coefficients. This is due to the low resolution of pressure measurements in small waves. Also, the linear approximation does not cross the flow rate axis at the origin. The reason for this discrepancy was not established. It is likely due to the limitations of experimental measurements at such a small scale. 
In a simplified approach, the linear coefficient $\mathrm{K}_{\mathrm{c}}$ may be used to compute the equivalent PTO damping and stiffness coefficients. The resulting calculations are presented in Table 8 for the period of resonance $\mathrm{T}=7 \mathrm{sec}$. Predicted applied damping from the PTO at the period of resonance varies between 20 and $40 \%$ depending on the constant $\mathrm{K}_{\mathrm{c}}$ considered.

Table 8: Equivalent damping at the period of resonance due to modeled turbine in experiments

\begin{tabular}{|l|r|r|r|}
\hline Wave Steepness H/L & \multicolumn{1}{|c|}{$1 / 80$} & \multicolumn{2}{c|}{$\mathbf{1 / 4 0}$} \\
\hline \multicolumn{1}{|c|}{ Value from: } & floating case & \multicolumn{1}{c|}{ fixed case } & floating case \\
\hline K contant & 200 & 690 & 300 \\
\hline Damping w/o carpet (\% critical) & $7 \%$ & $12 \%$ & $12 \%$ \\
\hline Calculated damping from carpet (kg/s) & $9.65 \mathrm{E}+05$ & $5.07 \mathrm{E}+05$ & $8.84 \mathrm{E}+05$ \\
\hline Damping with carpet (\% critical) & $37.0 \%$ & $19.4 \%$ & $33.8 \%$ \\
\hline Total added damping (\% critical) & $44.0 \%$ & $31.4 \%$ & $45.8 \%$ \\
\hline Total applied damping (kg/s) & $1.15 \mathrm{E}+06$ & $8.20 \mathrm{E}+05$ & $1.20 \mathrm{E}+06$ \\
\hline Associated Stiffness (N/m) & $1.10 \mathrm{E}+06$ & $1.59 \mathrm{E}+06$ & $2.10 \mathrm{E}+06$ \\
\hline
\end{tabular}

Figure 22 shows the measured RAOs of the pumping mode for different wave steepness H/L. Numerical results are obtained with damping and stiffness values adjusted at each frequency, corresponding to

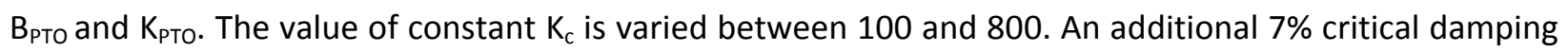
is added to model the non-linear damping in the case of $1 / 80$ wave steepness. The center of gravity is adjusted in the numerical results to best represent that of the experimental model. The lower values of $\mathrm{K}_{\mathrm{c}}$ match the experimental measurements best. A cancellation of the pumping RAO around $\mathrm{T}=12 \mathrm{sec}$ is not well represented by the model. This may indicate that the stiffness of the PTO is over-predicted.

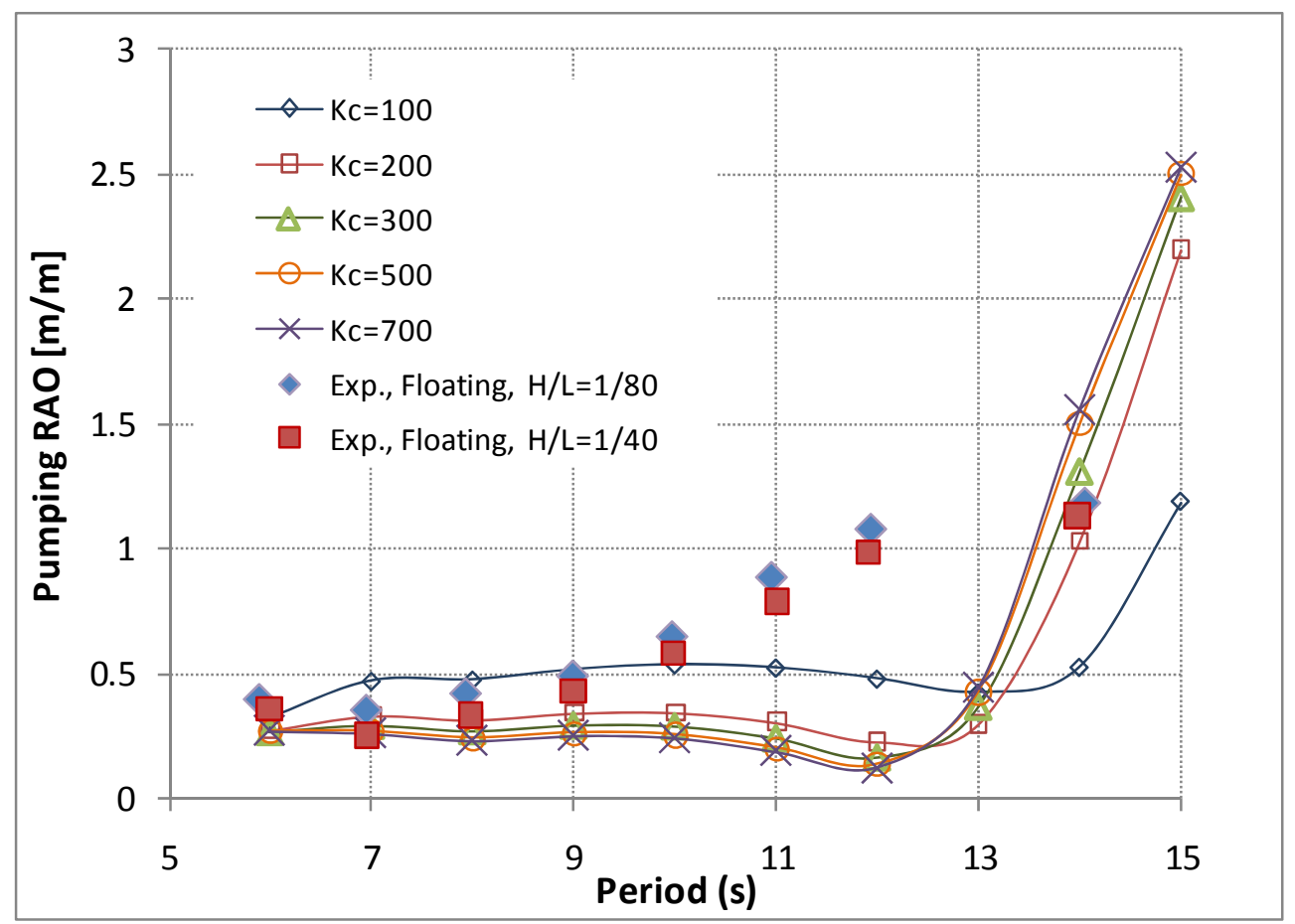

Figure 22: Pumping RAO [m/m] in damped cases on a floating structure (with a carpet Pumping mode in Global coordinates) 


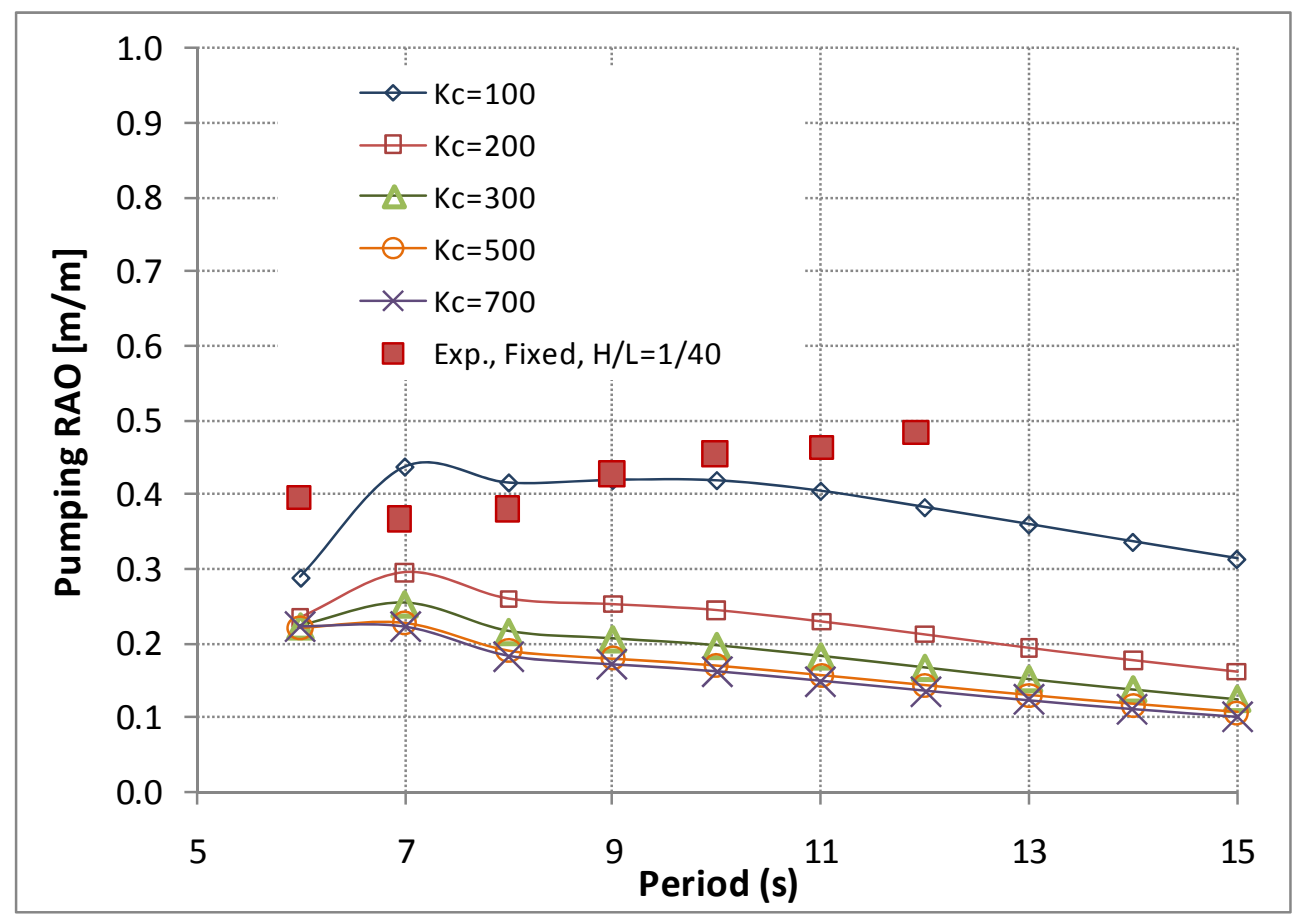

Figure 23: Pumping RAO [m/m] in damped cases (with a carpet) on the fixed structure

In experiments, at low frequencies, the pumping motion is much lower in the fixed case than in the floating case. This is predicted by linear theory. It is due to the effect of platform motion on the global free surface elevation. In Figure 23, the best match between numerical and experimental data is again observed at constant $\mathrm{K}_{\mathrm{c}}=100$.

\section{WindFloat RAOS Comparisons: Impact of the OWC on the WindFloat platform}

Linear theory predicts little effect of the OWC on the WindWaveFloat motions. To validate this assumption, platform motions were measured in the experiments with and without the OWC. Results with the OWC are compared when the chamber is open and when the carpet is used to model the turbine. Results are presented in Figure 24 to Figure 26 for surge, heave and pitch.

The surge motion of the structure is well predicted by WAMIT. Results show the OWC shell and the PTO have no effect on the surge motion of the platform.

Similarly, in heave, experiments show no difference between the base case, without the OWC, and the case with the OWC shell. The PTO may introduce some effect at lower frequency, around the period of resonance of the WindWaveFloat. A small difference is captured by WAMIT. However, due to non-linear effects of the water entrapment plates, WAMIT predicts a much steeper cancellation. 


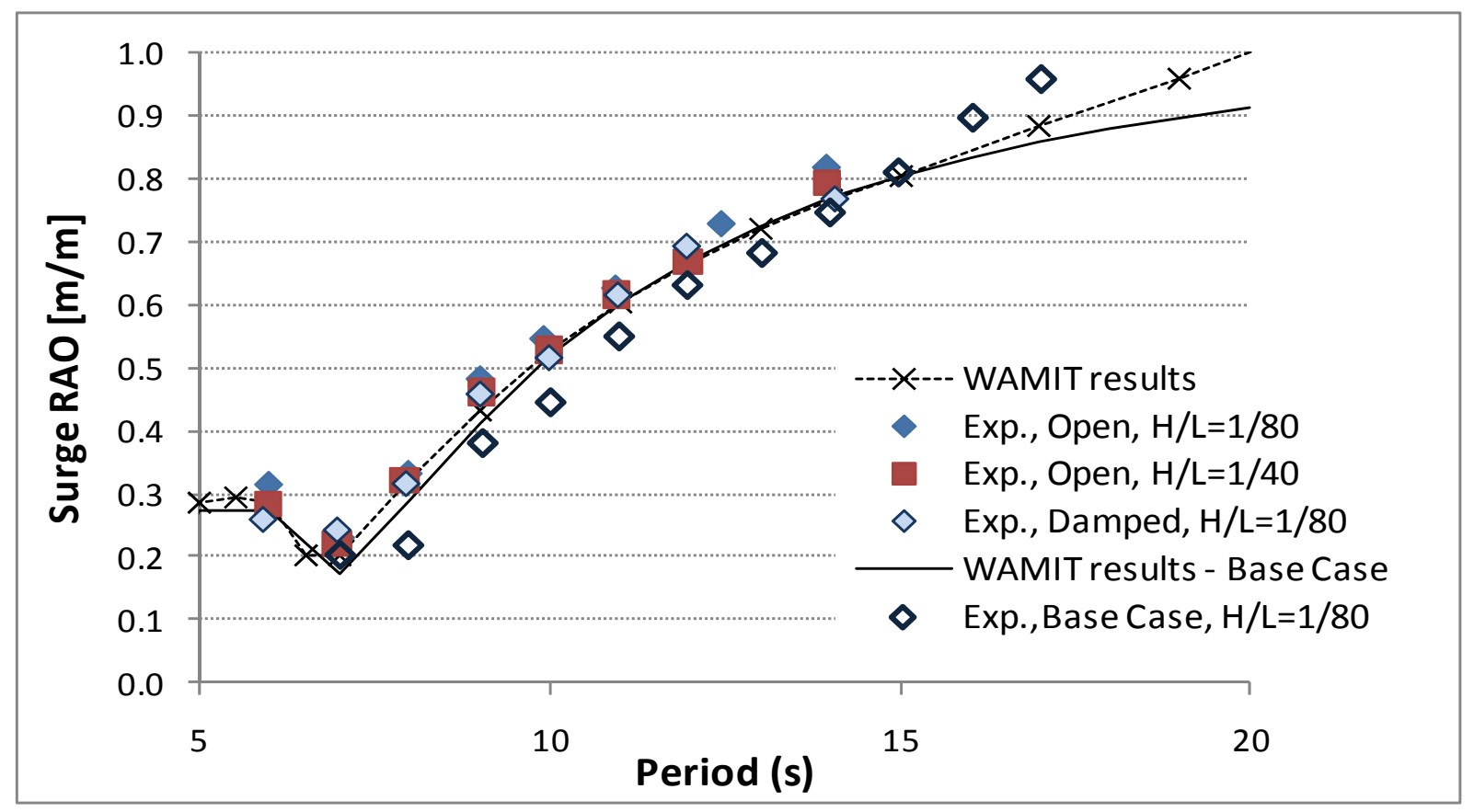

Figure 24: Comparison of Surge RAOs without the OWC (Base Case) and with the OWC

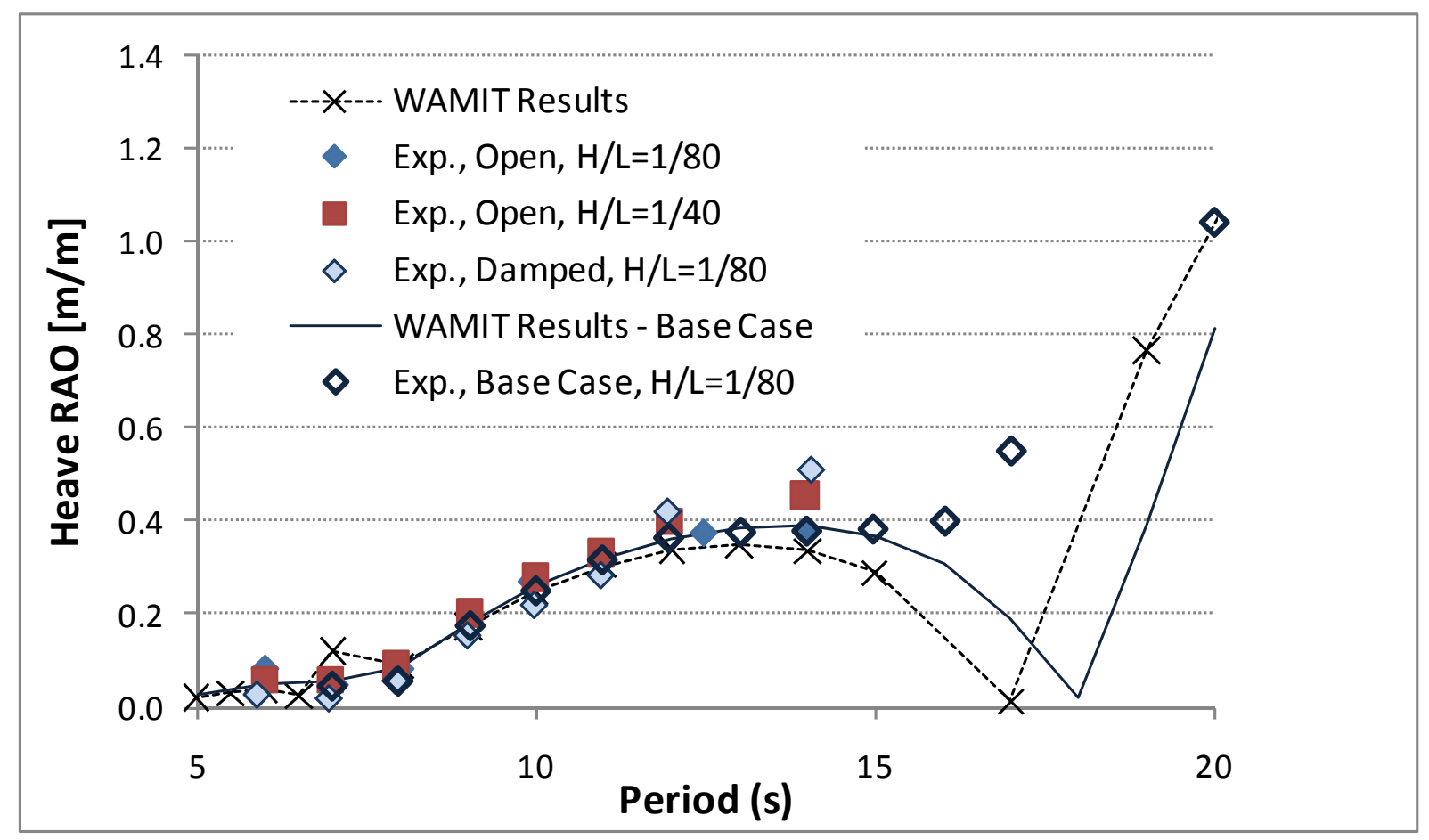

Figure 25: Comparison of Heave RAOs without the OWC (Base Case) and with the OWC 


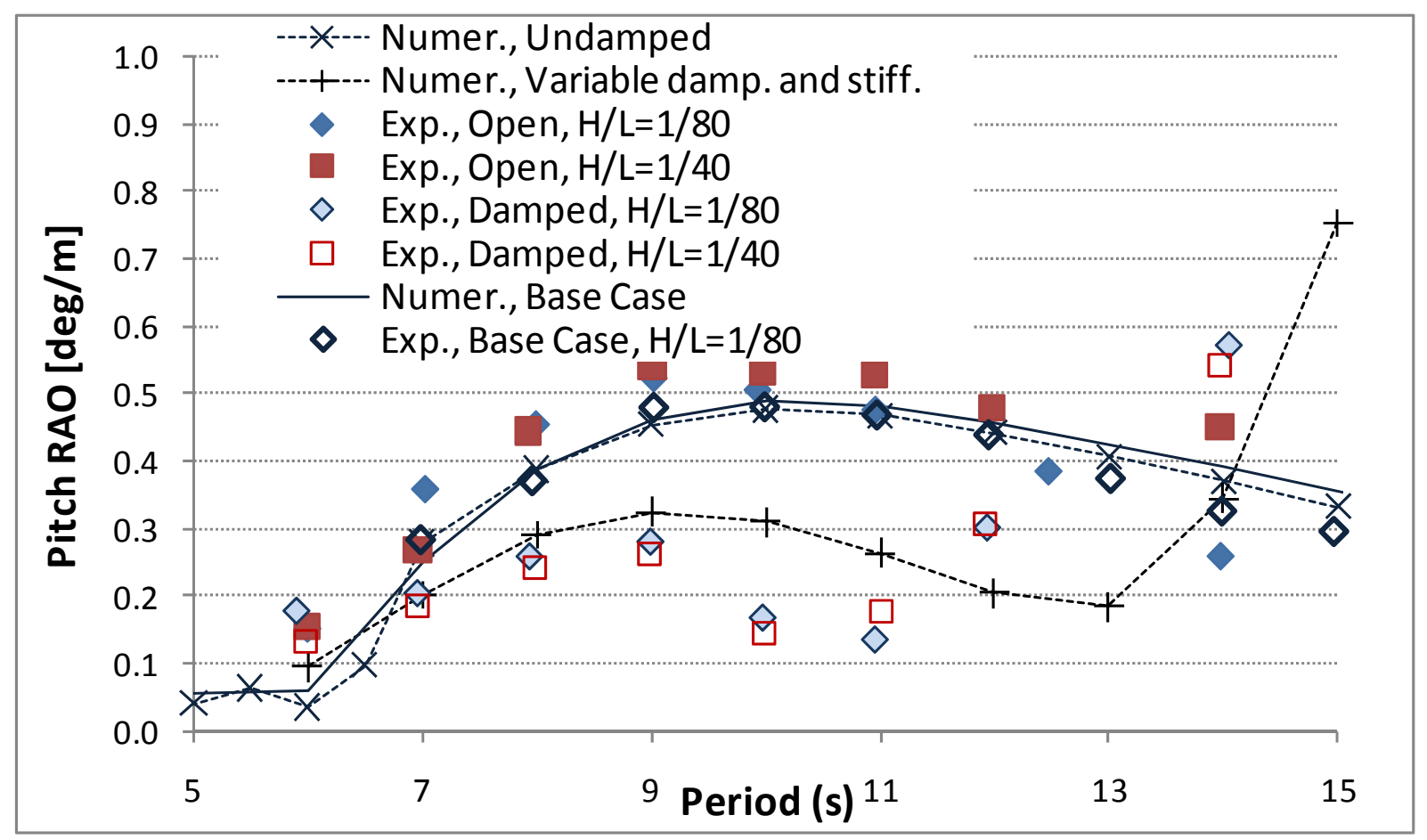

Figure 26: Comparison of Pitch RAOs without the OWC (Base Case) and with the OWC $\left(K_{c}=100\right.$ in numerical damped case)

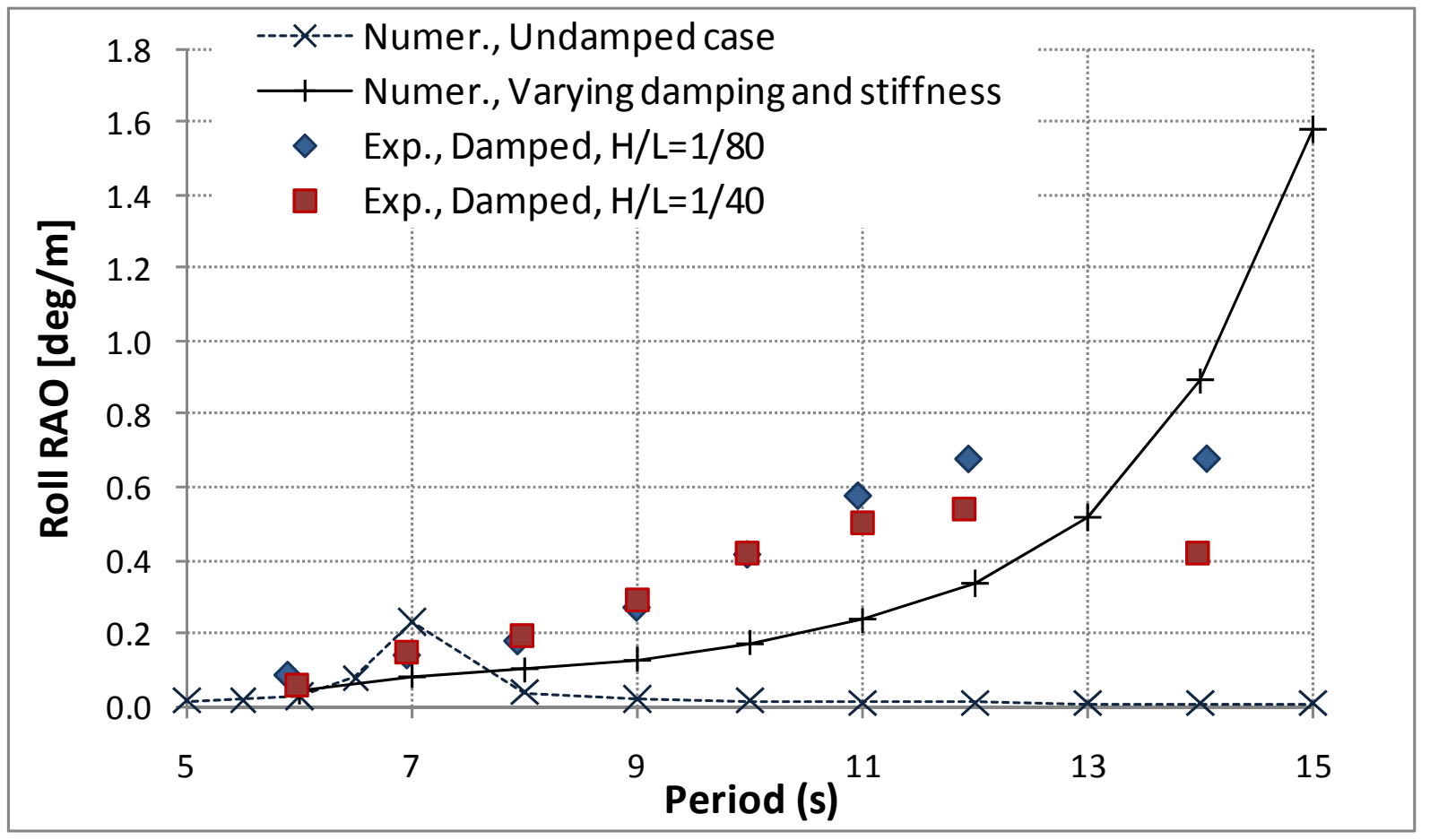

Figure 27: Comparison of Experimental Roll RAOs with a carpet damper and Numerical predictions $\left(K_{c}=100\right.$ in numerical damped case) 
Although the experimental results are identical in pitch in the base case and with the open OWC shell, observed pitch motions are very different once the damper is added to model the turbine. This phenomenon is predicted by linear diffraction-radiation theory when the equivalent PTO damping and stiffness coefficients are introduced. A similar effect is observed in roll, in Figure 27.

The roll is induced by the non-symmetrical PTO force on the platform. The damping term of the PTO force explains the reduction in amplitude of the pitch as observed in Figure 26.

\subsubsection{Power Prediction}

\section{Absorbed Power and Capture Width}

The instantaneous OWC power comprises both a resistive and a reactive term. The reactive term, related to the spring effect, is in quadrature of phase with the velocity and so it represents a flux of energy with zero mean. Thus, the only term contributing to the absorbed power is the damping force (resistive term) produced by the PTO (e.g. by the air turbine). Therefore the mean power absorbed over a wave period is:

$$
\overline{\mathrm{P}}_{\mathrm{abs}}=\frac{1}{2} \mathrm{~B}_{\mathrm{pto}}\left|\hat{\mathrm{u}}_{\mathrm{r}}\right|^{2}
$$

where $\hat{\mathrm{u}}_{\mathrm{r}}$ is the complex amplitude of the relative vertical velocity induced by the modes that contribute to the volume variation of the air chamber.

Similarly to the other devices, the capture width can be calculated as:

$$
L_{c a p}=\frac{\bar{P}_{a b s}}{P_{w}}=\frac{2 \omega^{3} B_{p t o}}{\rho g^{2}}\left|\frac{\hat{V}}{S A}\right|^{2} .
$$

where $\hat{\mathrm{V}}$ is the complex amplitude of the air volume oscillation and $\mathrm{S}$ is the area of the internal free surface.

The experimental capture width of the system, is computed with constant $K_{c}=100$. The capture width is normalized with the width of the OWC and results are compared to numerical results in Figure 28 and Figure 29. The platform motions affect the capture width at low frequencies, where the capture width of the fixed model decreases. The maximum measured capture width does not occur at the natural period of the system. That may be explained by the additional stiffness introduced by the PTO on the internal free surface pumping mode. This is consistent with the absence of resonance at the natural period in Figure 22. Additionally, the capture width is larger in less steep waves, which reflects increased losses through non-linear effects in steep waves. The discrepancies between numerical and experimental results in the floating cases may be linked to corresponding differences in predicted and measured roll and pitch angles of the platform. 


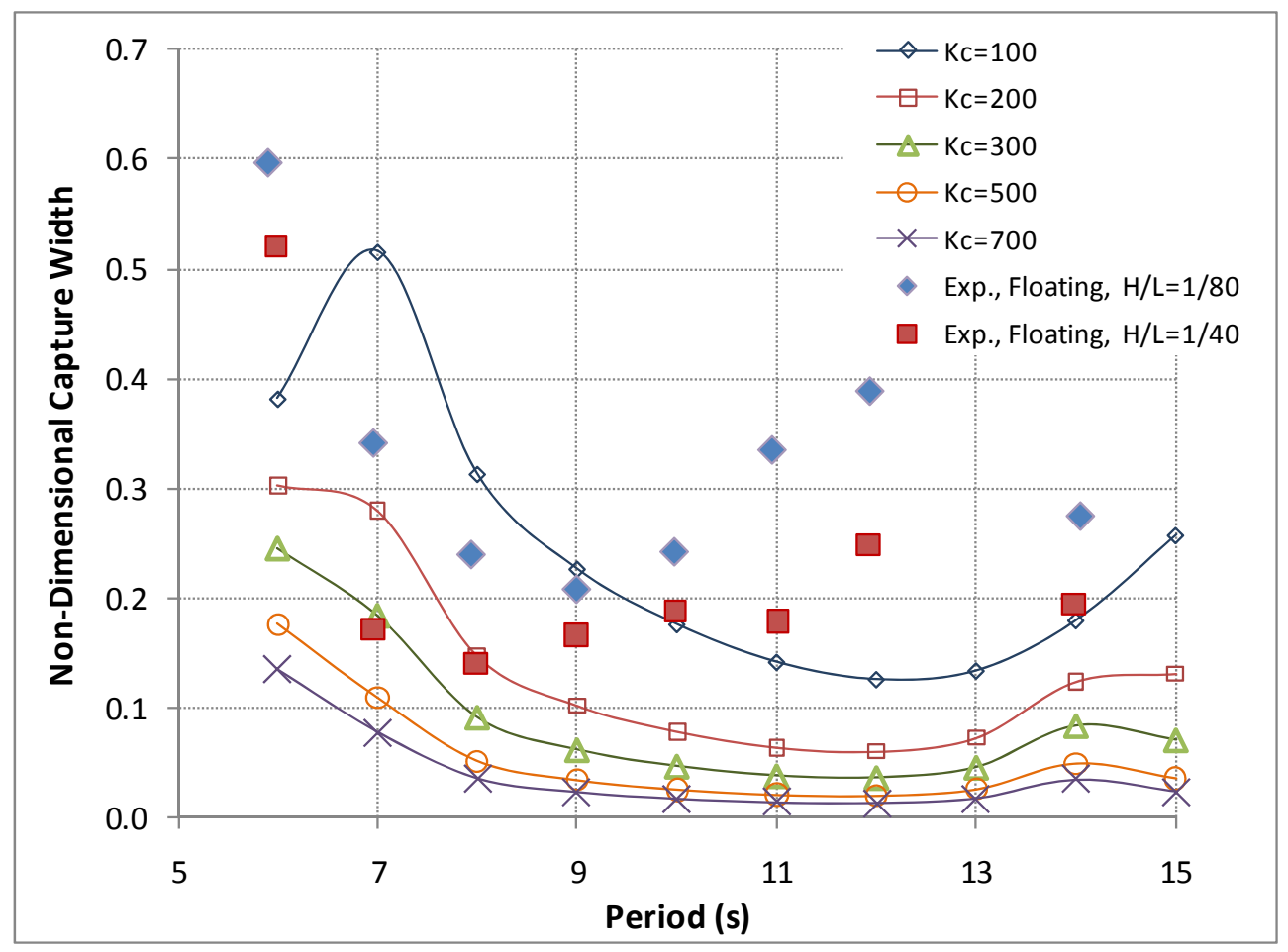

Figure 28: Comparison between Numerical (varying $K_{c}$ ) and Experimental $\left(K_{c}=100\right)$ NonDimensional Capture Width of the floating WindWaveFloat OWC

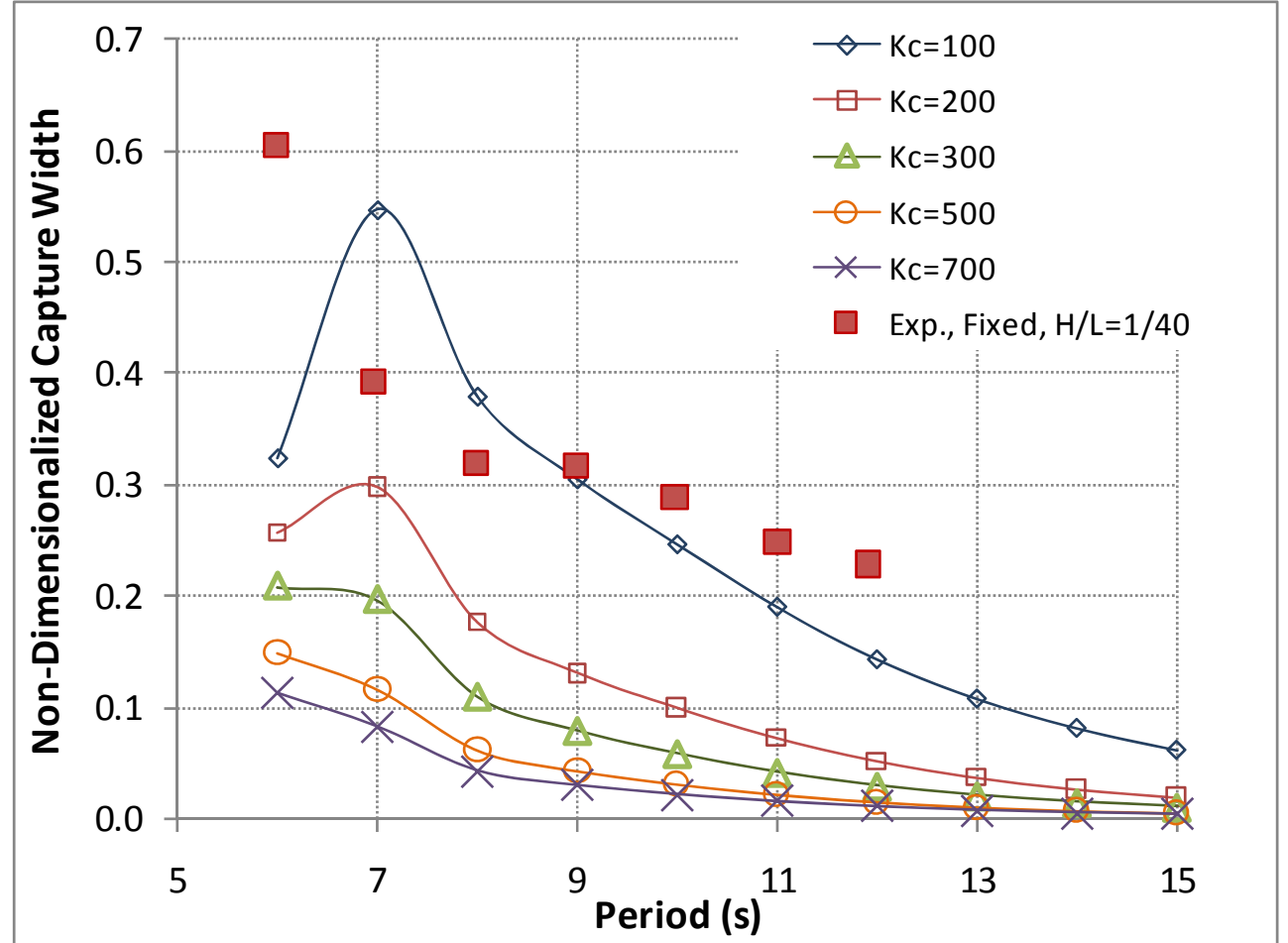

Figure 29: Comparison between Numerical (Varying $\left.K_{c}\right)$ and Experimental $\left(K_{c}=100\right)$ NonDimensional Capture Width of the fixed WindWaveFloat OWC 


\section{Wells turbine characteristics for maximum power extraction}

The PTO stiffness and damping coefficients are varied for different wave periods (at a given wave slope, thus at a given wave amplitude) to spot the optimal wave power extraction point, which corresponds to the wave and PTO characteristics generating as much mechanical power as possible and as efficiently as possible, thus which corresponds to the maximal capture width. After a couple of trial and errors, this optimal point is discovered and occurs for a wave of $5 \mathrm{~s}$, a stiffness coefficient of $200 \mathrm{kN} / \mathrm{s}$ and damping coefficient of 200 tonnes/s. For example, about $139 \mathrm{~kW}$ of average mechanical power would be harnessed with a regular wave height of $2 \mathrm{~m}$, which corresponds to a normalized capture width of 0.44 .

Table 9: Optimal operating point in regular waves

\begin{tabular}{|c|c|c|c|}
\hline & $\begin{array}{c}\text { Line Stiffness } \\
\text { coefficient } \\
(\mathbf{k N} / \mathrm{m})\end{array}$ & $\begin{array}{c}\text { Line Damping } \\
\text { coefficient (te/s) }\end{array}$ & $\begin{array}{c}\text { Maximum } \\
\text { Capture } \\
\text { Width }\end{array}$ \\
\hline $\begin{array}{c}\text { Maximum Power Output for one } \\
\text { wave period }\end{array}$ & 200 & 200 & 0.57 at T=5s \\
\hline
\end{tabular}

Power/Capture Width matrices for different wave heights and periods in regular waves 


\section{Conclusions}

In the framework of the WindWaveFloat project, a generic 5MW WindFloat platform is used as the structure supporting different wave-energy devices. In this report, for each of the different generic designs of the WindWaveFloat (SWEDE, OWSC, and OWC), numerical and experimental results of the systems are presented in regular wave conditions. In order to study the performance of these WindWaveFloat concepts, the primary objective of this work is to validate the numerical models created both in OrcaFlex and WAMIT (depending on the WEC) using experimental results obtained in a wave tank at model scale. After the numerical models are validated, the impact of the WECs on the WindFloat platform motions, and the wave energy extraction process, that is to say the conversion of wave energy into mechanical energy (or pneumatic energy in the case of the OWC), are evaluated for several stiffness and damping coefficients, modeling potential PTO characteristics in each case. The capture width is also computed and the optimal operating point is deduced by varying the PTO stiffness and damping coefficients for each of the generic WECs. Finally, a power and/or capture width matrix is derived at this optimal PTO operating point to estimate the average power output that can be harnessed in different regular waves.

A few elements presented in this report that should be highlighted are described as follows. Discrepancies between numerical models and experimental results often occur because of the presence of non-linear effects. It is important to understand these effects often linked to the quadratic damping of the system, in order to calibrate the numerical models and accurately estimate the performance of each of the device. For certain wave conditions, further experimental tests are thus needed in the future, especially if the waves and device motions are larger, and linear theory cannot be accurately employed. For the OWC in particular, the numerical model does not account for the non-linearity of the Wells turbine. These non-linearities could be introduced as an efficiency ratio. In a recent analysis, Martins-Rivas (2008) has included the effect of air compressibility. It results in a loss of extracted power. Losses in the Turbine-Generator system - including mechanical losses and electrical losses - were also neglected.

Most of the devices hardly affect the motions of the WindFloat platform, which confirms a minimal impact on the wind energy generation of the hybrid system, since platform displacements and rotations are kept small. In particular, the OWC even reduces the pitch motion of the WindFloat when PTO damping coefficients are used, but generates transverse roll motions that can probably be avoided, if a symmetrical configuration is used in the future. For all devices, a more extensive analysis, across a larger range of periods, PTO stiffness and damping coefficients should be carried out in the future to confirm this encouraging trend.

Another promising trend is the appearance of beneficial relative motions between wave-energy devices designed to resonate in the range of wave excitation frequencies (periods of $4 \mathrm{~s}$ to $18 \mathrm{~s}$ ), and the WindFloat designed to stay nearly still in this wave frequency range, but at a different phase, leading to higher relative motions between the two systems than in the case of a WEC attached to a fixed WindFloat, resulting in increased power output. For example, in the case of the SWEDE, the relative motion of the sphere is maximal with respect to the WindFloat motion at a period of $6 \mathrm{~s}$, leading to 
maximum tensions in the SWEDE lines. This favorable phenomenon enables a shift in maximum average mechanical power from the SWEDE heave resonance period ( $T=4 s$ here) to a slightly higher period $(T=6 s)$, which shifts the device energy absorption spectral bandwidth inside the wave energy spectral bandwidth.

The efficiency of the conversion of the harnessed mechanical energy (or pneumatic energy in the case of the OWC) into electrical energy is not taken into account, since the PTO has not been selected yet, except for the OWC which uses a bi-directional Wells turbine without non-linear effects. More studies need to be carried out to understand the impact of irregular waves vs. regular waves on the system. After the performance of the device is assessed in irregular wave, a PTO will be selected or designed to perform with the stiffness and damping characteristics of the optimal operating point.

In a future stage, the geometry of the wave-energy device might be optimized to improve the overall performance of the wave-energy device, but the suggested geometries for the generic devices already present acceptable power performances. 


\section{References}

[1] Henderson, R., (2006), "Design, Simulation, and Testing of a Novel Hydraulic Power Take-Off System for the Pelamis Wave Energy Converter”, Renewable Energy 31 (2006) 271-283

[2] Roddier D., Peiffer A., Aubault A., Weinstein J., "A Generic 5 MW Windfloat For Numerical Tool Validation \& Comparison against a Generic Spar", Proc. of OMAE 2011, Rotterdam, The Netherlands

[3] Jonkman J., Butterfield S., Musial W., and Scott G., "Definition of a 5-MW Reference Wind Turbine for offshore Development", NREL/TP-500-38060, February 2009

[4] Ballast, A., Zandbergen, P.J. ,"Fully Non-Linear Diffraction Calculations of a Floating Sphere in Regular Waves", IWWWFB, Norway, 2005

[5] Ruiz, M. T. , "Dynamics and hydrodynamics for floating wave energy converters”, Dissertação para obtenção do Grau de Mestre em Engenharia e Arquitectura Naval, Lisbon, December 2010

[6] Aubault A., Alves M., Sarmento A., Roddier D., Peiffer A. "Design of an Oscillating Water Column around the Columns of the WindFloat.", Proc. of OMAE 2011, Rotterdam, The Netherlands

[7] Roddier D., Cermelli C., Aubault A., Weinstein A. "WindFloat: A Floating Foundation for Offshore Wind Turbines", Journal of Renewable and Sustainable Energy, Vol.2, Issue 3, 2010

[8] OrcaFlex User Manual, Ulverston, UK. Last accessed in January 2011. www.orcina.com/SoftwareProducts/OrcaFlex/.../OrcaFlex.pdf

[9] WAMIT User Manual version 6.0, WAMIT Inc., MA, USA. Last accessed in January 2011. http://www.wamit.com/manual.htm

[10] Yeung R. W., Peiffer A., Tom N., Matlak T. , "Design, Analysis and Evaluation of the UC-Berkeley WaveEnergy Extractor", Proc of OMAE 2010, Shanghai, China

[11]Frischholz R., Wittenberg T., "Computer Aided Visual Motion Analysis", Advances in Quantitative Laryngoscopy using Motion-, Image- and Signal Analysis, Erlangen, 1997

[12] Newman, J.N. "Marine Hydrodynamics", The MIT press Cambridge, Massachusetts, USA and London, England

[13] Evans D V "Some analytic results for 2D and 3D wave, energy absorbers" pp. 213-249 in "Power from Sea Waves" Ed. Count, B M, Ac. Press 1980

[14] “Marine Energy Challenge, Marine Energy Glossary”, Entec, Carbon Trust Report, 2005

[15]M. Alves, T. Soulard and A.J.N.A. Sarmento "Force Reacting Principle Applied to a Heave Point Absorber Wave Energy Converter". Proc of the 19th International Offshore and Polar Eng Conf, Osaka, Japan, ISOPE, Vol I, pp 312-318, 2009.

[16] Brito-Melo, A. and Sarmento, A.J.N.A., "Numerical Modelling of OWC wave-power plants of the oscillating water column type", Advances in Boundary Elements, WIT Press, pp 25-34, 2002

[17]Brito-Melo, A., Gato L.M.C. and Sarmento A.J.N.A., "Analysis of Wells Turbine Design Parameters by Numerical Simulaiton of the OWC Performance", Ocean Engineering, Vol. 29, Issue 12, pp.1463-1477, 2002

[18] C.A. Cermelli, D.G. Roddier, "Experimental and numerical investigation of the stabilizing effects of a waterentrapment plate on a deepwater minimal floating platform“, Proc. 24th International Conference on offshore Mechanics and Arctic Engineering, Halkidiki, Greece, June 2005 
[19] C. Cermelli, D. Roddier, A. Aubault, WindFloat: a Floating Foundation for Offshore Wind Turbines Part II: Hydrodynamics, Procs. of OMAE'09, 28th International Conference on Offshore Mechanics and Arctic Engineering, Honolulu, HI, USA May 31, Jun 5, 2009

[20]Curran R., Stewart T.P. and Whittaker T.J.T., "Design Synthesis of Oscillating Water Column Wave Energy Converters: Performance Matching", Proc. Instn Mech Engrs, Vol.211, Part A, Pp.489-505

[21] FalCĂO, A.F.O. and Rodrigues, R.J.A., "Stochastic Modeling of OWC Wave Power Plant Performance", Applied Ocean Research, Vol.24, 2002

[22]Falnes, J., Ocean Waves and Oscillating Systems, 2002, Norwegian University of Science and Technology, Trondheim

[23] L.M.C. Gato and A.F. de O. Falcăo, "Aerodynamics of the Wells Turbine”, Int. J. Mech. Sci., Vol. 30, No.6, PP.383-395. 1988

[24]Martins-rivas, h and Mei, C.C., "Wave Power extraction from an Oscillating Water column along a STRAIGHT COAST", OCEAN ENGINEERING, VOL. 36, PP.426-433, 2009

[25] Newman, J.N." Wave Effects on Deformable Bodies", Applied Ocean Research, Vol.16, Pp.47-59, 1994

[26] Ragunathan S., "The Wells Air Turbine for Wave Energy Conversion", Prog. Aerospace Sci., Vol.31, pp. 335386,1995

[27]D. Roddier, A. Peiffer, J. Weinstein, A. Aubault, “A Generic 5MW WindFloat for Numerical Tool Validation \& Comparison against a Generic 5MW Spar”, OMAE, 2011

[28]D. Roddier, C. Cermelli, A. Aubault, A. Weinstein, "WindFloat: A Floating Foundation for Offshore Wind Turbines", Journal of Renewable and Sustainable Energy, Vol.2, Issue 3, 2010

[29] Wayman, E. N., Sclavounos, P. D., Butterfield, S., Jonkman, J., and Musial, W., "Coupled Dynamic Modeling of Floating Wind Turbine Systems," 2006 Offshore Technology Conference, 1-4 May 2006, Houston, TX 


\section{A Technical background}

Through the dissemination task of this DOE grant, three papers will be presented at OMAE 2011 in Rotterdam. These papers are included here, and form the technical basis of this report. The OWSC work was not published yet, however the methodology and tools used for this design are very similar than for the FLAPS.

\section{A.1. OMAE2011-50278: A Generic 5 MW WindFloat for Numerical Tool Validations}

This paper describes the WindFloat base case experiments and computations that were performed on the WindFloat alone. These are important to understand the influence of the additional WEC. This generic design was fitted with the NREL $5 \mathrm{MW}$ generic wind turbine.

\section{A.2. OMAE2011-49014: A Generic 5 MW WindFloat for Numerical Tool Validations}

This paper describes the oscillating water column experiments and computations that were performed on the WindFloat. This work was performed in cooperation with the Portuguese Wave Energy Center (WavEC). WavEC has worked on multiple oscillating Water columns, including the Pico Plant. Two independent models were used to ensure the validity of the methodology, especially in the modeling of the turbine.

\section{A.3. OMAE2011-49015: Design of a Point Absorber inside the WindFloat Structure}

This paper describes the point energy absorber placed inside the triangle formed by the WindFloat columns. The numerical tool OrcaFlex was used to predict the motions and calculate the power output. Again, the numerical models were validated against similar experiments. 
OMAE2011-49014

\section{MODELING OF AN OSCILLATING WATER COLUMN ON THE FLOATING FOUNDATION WINDFLOAT}

\author{
Alexia Aubault \\ Marine Innovation \& Technology \\ Berkeley, CA \\ António Sarmento \\ Technical University of Lisbon \\ Lisbon, Portugal
}

\author{
Dominique Roddier \\ Principal Power Inc \\ Berkeley, CA
}

\author{
Marco Alves \\ Wave Energy Centre \\ Lisbon, Portugal
}

\author{
Antoine Peiffer \\ Marine Innovation \& Technology \\ Berkeley, CA
}

\begin{abstract}
This paper summarizes the theory behind the modeling that was performed to incorporate an oscillating- water-column type Wave energy Converter (WEC) into the WindFloat hull. The WindFloat is a floating structure supporting a very large $(>5 \mathrm{MW})$ wind turbine. By adding a WEC to the structure, the overall economic cost of the project can be improved by sharing both mooring and power infrastructure. A numerical model was developed using the diffraction-radiation code WAMIT and assuming as PTO equipment, a generic wells turbine. It is important to model the turbine accurately, to understand the power capacity of the device. Details on the modeling of the system are discussed and numerical results and compared against experiments as a validation of the model. The effect of coupling between the floating foundation of the WindFloat and the OWC is investigated thoroughly.
\end{abstract}

\section{KEYWORDS}

Ocean Renewable Energy, Floating Foundation for Wind Turbines, Offshore Wind, Wave Energy, Oscillating Water Column

\section{INTRODUCTION}

The main challenge for offshore floating renewable energy devices remains to build a structure capable of withstanding the challenging ocean environment while financially viable in a competitive global energy market. Due to technological similarities to onshore wind energy, the offshore wind industry is developing quickly. The WindFloat, a floating foundation for multi- megawattwind turbines, is a three-column semisubmersible platform designed for world-wide environments. It is designed to allow wind energy harvesting in waterdepth of 40 meters and above, where fixed offshore turbines cease being economical. The wind turbine is typically a horizontal-axis turbine at the top of a tower, on one of the three columns. In this configuration, the WindFloat has enough deck space for additional equipment. In a proposed application, wave energy converters could be installed on the floater - which is then referred to as a WindWaveFloat.

The concentration of several devices on one platform has both economic and operational advantages. Wind and wave energy converters can share the electrical cable and power transfer equipment to transport the electricity to shore. Capital costs are also reduced overall provided that the design of the foundation can be adapted to multiple devices with minimum modifications. Access to multiple devices would be simplified, resulting in cost-saving at the operational level.

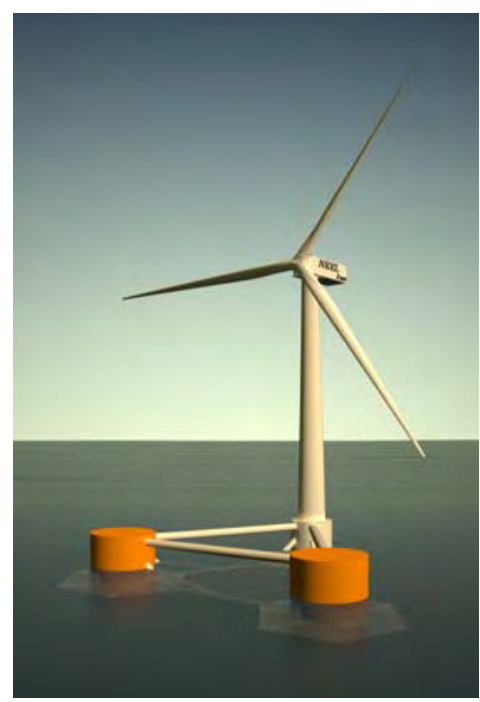

Figure 1: WindWaveFloat with two OWC Chambers 
Most wave energy converters pertain to one of three categories: oscillating water column, single or multiple point absorbers, oscillating wave surge converter. Generic wave energy converters of each category are considered and studied independently. This paper focuses on the design of a WindWaveFloat with an oscillating water column (OWC). In an oscillating water column (OWC), water enters through a subsurface opening into a chamber that contains air. The wave action causes the captured water column to rise and fall like a piston, compressing and decompressing the air. As a result, there is an air flow moving back and forth through a turbine coupled to an electric generator.

To minimize the disturbance to the initial WindFloat design, the chamber is built externally around the two columns not supporting the turbine. In this case, the air turbine is a bidirectional Wells turbine, which is the most common in this type of application. Other turbines such as air-impulse turbines have been considered for such applications in recent studies.

The assessment of the successful integration of an OWC on the WindWaveFloat should take into account:

- The effect of the OWC on the platform motions, which in turn may affect the performance of the wind turbine. The wind energy converter is especially sensitive to angular motions.

- The effect of platform motions on the performance of the wave energy device. This latter point has previously been studied in the case of floating OWCs, such as the Oceanlinx systems.

A proposed design of the WindWaveFloat with OWC is presented herein. Numerical tools are developed in the frequency domain to analyze the coupled effects of the OWC and the floating platform based on linear theory. Extensive work was carried out on the numerical modeling of the Wells turbine. A model of the power-take-off is developed based on previous work on Wells turbine, such as Gato \& Falcăo (1988) and Ragunathan (1995). Linear theory is used to calculate the power output of the wave energy converter. Model tests are carried out on this design to validate the numerical analysis.

\section{DESCRIPTION OF WIND-WAVE FLOAT WITH AN OSCILLATING WATER COLUMN (WWF-OWC)}

The WindWaveFloat foundation used in this analysis is identical to that designed to support a 5MW NREL wind turbine. The characteristics of the hull and mooring of this reference WindFloat are discussed thoroughly by Roddier et al. (OMAE 2011).

The WindWaveFloat has 3 cylindrical columns connected through a tubular truss to transfer lateral loads. At the base of each column, a hexagonal heave plate controls the waveinduced motions of the structure by increasing the added mass and damping. The hydrodynamic behavior and the global loads on the WindFloat have been the object of previous publications. This paper focuses on the variations of hydrodynamic behavior due to the introduction of the oscillating water column.

The oscillating water column is fitted on one of the spare columns of the Wind-Wave-Float. The chamber is of annular shape, between an external shell and the column, so that the foundation columns are not modified. To simplify the geometry, it encompasses only 240 degrees around the column and remains clear of the truss tubular, as illustrated in a top view in Figure 2. The chamber extends straight up to the top of column and its draft can vary. The base case of this analysis is a $9 \mathrm{~m}$ draft.

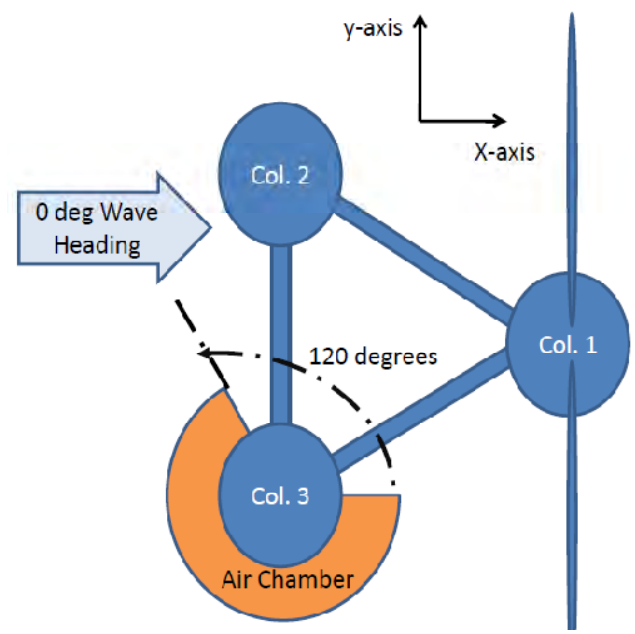

Figure 2: Configuration of OWC on WindWaveFloat - Top View

Table 1 provides the main dimensions of the reference WindWave-Float.

Table 1: WindFloat Main Particulars

\begin{tabular}{|l|c|c|}
\hline \multicolumn{2}{|c|}{ WindFloat 5 MW Main Dimensions } & Units \\
\hline \hline Column diameter & 10.0 & $\mathrm{~m}$ \\
\hline Length of water entrapment plate edge & 15.0 & $\mathrm{~m}$ \\
\hline Column center to center & 46.0 & $\mathrm{M}$ \\
\hline Operating draft & 17.0 & $\mathrm{~m}$ \\
\hline Airgap & 10.0 & $\mathrm{~m}$ \\
\hline OWC external diameter & 18.0 & $\mathrm{~m}$ \\
\hline OWC draft & 9.0 & $\mathrm{~m}$ \\
\hline
\end{tabular}

A Wells turbine is used to extract the power from the air chamber. The Wells turbine is a low pressure air turbine with symmetrical airfoils which rotates in the same direction regardless of the direction of the incident airflow. Prof. A.A. Wells developed this turbine specifically for direction-changing airflows such as the wave motion induced airflow in an OWC. Extensive research has been done on the behavior and design of the Wells turbine. Wells turbines are used at a number of OWC power plants worldwide, including at the LIMPET $500 \mathrm{~kW}$ 
plant on the Island of Islay, in Scotland and at the European OWC Wave Power Plant, on the Island of Pico in the Azores.

A Wells turbine is defined by geometric properties, such as solidity $\mathrm{S}$ - total blade area to turbine swept area ratio -, radius $\mathrm{R}$, the outer radius of the blades, swept blade area $\mathrm{A}_{\mathrm{T}}$.

These properties may be directly related to the turbine characteristics which govern the coupling between the OWC chamber and Power-Take-Off. In the present analysis a turbine radius of $1 \mathrm{~m}$ is used. Other parameters should be optimized for best performance. This optimization is outside the scope of this paper, but several values will be used to determine the effects of turbine on the OWC behavior and power output.

\section{THEORETICAL BACKGROUND}

The motion of the internal free surface of the WindWaveFloat OWC for unidirectional waves can be approximated by the decomposition into a vertical uniform motion, called pumping mode, and a linearly-varying sloshing motion. The sloshing motion coordinate is centered at the longitudinal centroid of the internal free surface of the OWC. Only waves moving in the direction of the $\mathrm{x}$-axis, as defined in Figure 2, are considered in this analysis, to limit the number of sloshing modes to 1 . In this particular device the pumping mode is dominant since the chamber diameter is smaller compared to the wavelength of the typical incident waves.

Assuming that the waves and the device oscillations are described by small amplitude motions, the hydrodynamic problem is well characterized by a linear approach. In this context, the 8-degree of freedom (DoF) system of motion equations, in the frequency domain, is written as:

$-\omega^{2}[M+A(\omega)] \hat{x}+i \omega B(\omega) \hat{x}+C \hat{x}=\hat{F}(\omega)+\hat{F}_{p t o}(\omega)$

where $\mathrm{x}$ is the 8 -DoF displacement vector including internal free surface sloshing and pumping, $M$ the $8 \times 8$ mass matrix of the WindWaveFloat-OWC, A the $8 \times 8$ frequency-dependent added mass matrix, $\mathrm{B}$ the $8 \times 8$ frequency-dependent damping matrix and $\mathrm{C}$ the $8 \times 8$ stiffness matrix. $\mathrm{F}$ and $\mathrm{F}_{\mathrm{pto}}$ represent, respectively, the incident wave excitation force and the load induced by the Power-Take-Off (PTO) equipment (the hat symbol, $\wedge$, denote complex amplitude).

The matrix $\mathrm{C}$ includes the hydrostatic stiffness $\mathrm{K}_{\mathrm{H}}$ and the linearized mooring stiffness $\mathrm{K}_{\mathrm{M}}$. The damping matrix comprises the hydrodynamic damping, $\mathrm{B}_{\mathrm{w}}$, the linearized viscous damping, $\mathrm{B}_{\mathrm{v}}$, and a linearized damping due to head losses in the air flow inside the chamber, $B_{c}$, i.e,

$\mathrm{B}=\mathrm{B}_{\mathrm{w}}+\mathrm{B}_{\mathrm{v}}+\mathrm{B}_{\mathrm{c}}$

The effect of the PTO on the overall dynamics is represented as an external force. If the internal free surface behaves like a piston, the pressure can be assumed uniform inside the chamber. Thus, this external force is given by:

$F_{p t o}(\omega)=-p(\omega) S$

in which $\mathrm{p}$ is the pressure drop across the turbine and $\mathrm{S}$ is the area of the internal free surface.

If the air is assumed to be an ideal gas and the air compression and decompression an isentropic process, the time-dependent air density is described by:

$\rho=\rho_{0}\left(\frac{p}{p_{0}}\right)^{1 / \gamma}$,

where the density, $\rho_{0}$, and the pressure, $\mathrm{p}_{0}$, describe the state of the chamber at rest and $\gamma$ represents the heat capacity ratio of air. Linearizing the time derivative of Eq. 4 it results:

$\dot{\rho}=\frac{\rho_{0}}{\gamma \mathrm{p}_{0}} \dot{\mathrm{p}}$

Then, the linearized mass flow through the turbine may be obtained from,

$\dot{\mathrm{m}}=\frac{\mathrm{d}(\rho \mathrm{V})}{\mathrm{dt}}=\frac{\rho_{0}}{\gamma \mathrm{p}_{0}} \dot{\mathrm{p}} \mathrm{V}_{0}+\rho_{0} \dot{\mathrm{V}}$,

where $\mathrm{V}$ the volume of air in the chamber.

Using non-dimensional turbo-machinery nomenclature, a Wells turbine of diameter $\mathrm{D}$ and rotational velocity $\mathrm{N}$ is characterized by a linear relation between the pressure and flow coefficients:

$\Psi=K \Phi$

Where the pressure coefficient is defined by:

$\psi=\frac{p}{\rho_{o} N^{2} D^{2}}$

And the flow coefficient by:

$\Phi=\frac{\dot{m}}{\rho_{0} N D^{3}}$

To simulate experimentally a Wells turbine a permeable carpet was used to introduce a pressure drop proportional to the flow rate. Thus, in this particular context an alternative nondimensionalization is used and the linear relation is expressed by: 
$\Psi_{c}=K_{c} \Phi_{c}$

where the pressure coefficient $\Psi_{\mathrm{c}}$ and flow coefficient $\Phi_{\mathrm{c}}$ are:

$\psi_{c}=\frac{p}{\rho_{0} g H}$

and

$\Phi_{c}=\frac{2 \pi \dot{m}}{\rho_{0} \omega S H}$

$\mathrm{H}$ is the wave height. Thus, introducing Eq. 7, 8 and 9 into Eq. 6 , the mass flow across the turbine may also be written as follows:

$\dot{m}=\frac{\omega S}{2 \pi g K_{c}} p$

Then, combining Eq. 10 and 6, it can be shown that, in the frequency domain, the pressure complex amplitude acquires the form

$$
\hat{p}=i \omega \frac{\Gamma}{\omega\left[1+(\varepsilon \Gamma)^{2}\right]} \frac{1}{S} \hat{V}-\omega^{2} \frac{\varepsilon \Gamma^{2}}{\omega\left[1+(\varepsilon \Gamma)^{2}\right]} \frac{1}{S} \hat{V}
$$

where $\hat{\mathrm{V}}$ is the complex amplitude of the air volume oscillation and the constants $\Gamma$ and $\varepsilon$ are defined by

$\Gamma=2 \pi \rho_{0} g K_{c}$

and

$\varepsilon=\frac{\mathrm{V}_{0}}{\gamma \mathrm{p}_{0} \mathrm{~S}}$.

In accordance with Eq. 3 and 11, the PTO force may be written by:

$\hat{\mathrm{F}}_{\mathrm{pto}}=-\mathrm{i} \omega \mathrm{B}_{\mathrm{pto}} \hat{\mathrm{x}}_{\mathrm{r}}+\omega^{2} \mathrm{~K}_{\mathrm{pto}} \hat{\mathrm{x}}_{\mathrm{r}}$

Or

$\hat{\mathrm{F}}_{\text {pto }}=-i \omega \frac{\mathrm{B}_{\text {pto }}}{\mathrm{S}} \hat{\mathrm{V}}+\omega^{2} \frac{\mathrm{K}_{\text {pto }}}{\mathrm{S}} \hat{\mathrm{V}}$,

where $\hat{x}_{r}$ is the complex amplitude of the relative displacement. Eq. 14a shows that the linear load induced by the PTO comprises a dissipative term proportional to the relative velocity and a reactive term due to the compression of air in the chamber and proportional to the relative acceleration. Likewise, dividing the damping, $\mathrm{B}_{\mathrm{pto}}$, and spring $\mathrm{K}_{\text {pto }}$ coefficients by the area of the internal free surface, $\mathrm{S}$; the dissipative term becomes proportional to the volume variation and the reactive term proportional to the rate of volume change, as Eq. 14b illustrates. According to Eq. 11, the PTO damping and spring coefficients result, respectively, from

$$
B_{p t o}=\frac{S \Gamma}{\omega\left[1+(\varepsilon \Gamma)^{2}\right]}
$$

and

$$
K_{p t o}=\frac{S c \Gamma^{2}}{\omega^{2}\left[1+(d \Gamma)^{2}\right]} .
$$

Finally, the system of motion equations for the 8-DoF WindWaveFloat device, given by Eq.1, may be rewritten, in the frequency domain, by

$$
\left[-\omega^{2}(M+A)+\left(C+K_{p t o}\right)\right] \hat{x}+i \omega\left(B+B_{p t o}\right) \hat{x}=\hat{F}
$$

From Eq. 17, the complex amplitudes of the platform and the free surface displacements can be computed, and so, the mass flow through the turbine inferred. Assuming uniform pressure inside the chamber, the sloshing mode has no effect on the volumetric oscilations. Moreover, the platform motions in the horizontal plane do not affect the volume of air in the chamber. Therefore, besides the piston mode, $\mathrm{x}_{7}$, only the structure heave, $x_{3}$, and pitch and roll motions contribute to the volumetric variations. So, the time derivative of the volume results from

$\dot{\mathrm{V}}=\mathrm{S} \dot{\mathrm{x}}_{7}-\mathrm{S} \dot{\mathrm{x}}_{3}+\bar{\xi} \mathrm{S} \dot{\mathrm{x}}_{5} \cos \left(\mathrm{x}_{5}\right)$,

where $\bar{\xi}$ is the average horizontal coordinate of the chamber in the $\mathrm{x}$-direction, in the global coordinate system, located at the center of the platform. In the frequency domain, the complex amplitude of the volumetric variation, $\dot{V}$, assumes the form:

$\hat{\phi}=\mathrm{i} \omega \mathrm{S}\left(\hat{\mathrm{x}}_{7}-\hat{\mathrm{x}}_{3}+\bar{\xi} \hat{\mathrm{x}}_{5}\right)$.

By means of Eq. 18 or 19 it is possible to verify that the PTO influences all rigid-motions which induce vertical motions on the chamber, in addition to the internal free surface pumping mode. Note that, to simplify Eq. 19 and the application of PTO damping, mode 7 can be defined relative to the vertical motion of the platform at the centroid of the internal free surface, as is the case in WAMIT. Roll motion can be introduced in the equations above similarly to pitch.

\section{POWER ABSORTION}

The instantaneous OWC power comprises both a resistive and a reactive term. The reactive term, related to the spring effect, is in quadrature of phase with the velocity and so it represents a flux of energy with zero mean. Thus, the only term contributing to the absorbed power is the damping force (resistive term) produced by the PTO (e.g. by the rotor). Therefore the mean power absorbed over a wave period is

$\overline{\mathrm{P}}_{\mathrm{abs}}=\frac{1}{2} \mathrm{~B}_{\mathrm{pto}}\left|\hat{\mathrm{u}}_{\mathrm{r}}\right|^{2}$

where $\hat{\mathrm{u}}_{\mathrm{r}}$ is the complex amplitude of the relative vertical velocity induced by the modes that contribute to the volume variation of the air chamber, i.e. 
$\hat{\mathrm{u}}_{\mathrm{r}}=\mathrm{i} \omega \hat{\mathrm{x}}_{\mathrm{r}}=\mathrm{i} \omega\left(\hat{\mathrm{x}}_{7}-\hat{\mathrm{x}}_{3}+\bar{\xi} \hat{\mathrm{x}}_{5}\right)$.

Hence, combining Eq. 19 and Eq. 20, the mean power absorbed results from

$$
\overline{\mathrm{P}}_{\mathrm{abs}}=\frac{1}{2 \mathrm{~S}^{2}} \mathrm{~B}_{\mathrm{pto}}|\hat{\phi}|^{2}=\frac{1}{2 \mathrm{~S}^{2}} \mathrm{~B}_{\mathrm{pto}} \omega^{2}|\hat{\mathrm{V}}|^{2} \text {. }
$$

Finally, in order to quantify the efficiency of the device, the capture width, $L$, is computed, being defined by

$\mathrm{L}_{\text {cap }}=\frac{\overline{\mathrm{P}}_{\mathrm{abs}}}{\mathrm{P}_{\mathrm{w}}}$,

where, $P_{w}$, represents the mean incident wave power per unit wave frontage. Using the dispersion relation $v_{g}=\omega / 2 k=g / 2 \omega$ valid for regular waves of amplitude A and frequency $\omega$ in deep water, $P_{w}$ for an incident wave is given by:

$\mathrm{P}_{\mathrm{w}}=\frac{1}{2} \rho g \mathrm{~A}^{2} v_{\mathrm{g}}=\frac{1}{4 \omega} \rho \mathrm{g}^{2} \mathrm{~A}^{2}$

Introducing Eq. 22 and 24 into Eq. 23, the capture width becomes

$L_{\text {cap }}=\frac{\bar{P}_{a b s}}{P_{w}}=\frac{2 \omega^{3} B_{p t o}}{\rho g^{2}}\left|\frac{\hat{V}}{S A}\right|^{2}$.

\section{NUMERICAL MODELING}

Response Amplitude Operators (RAOs) in all degrees of freedom are computed in the frequency domain based on Eq. 8 .

A numerical model of the WindWaveFloat with OWC is built in the frequency domain.

Linear radiation-diffraction software WAMIT is used. A highorder B-spline representation of the submerged hull of the WindWaveFloat and OWC is generated. The thin plates - water entrapment plates at the base of columns and shell of the OWC - are represented with dipoles. The internal free surface is represented with a B-spline patch at the waterline, to model its degrees of freedom.

The model of the platform is based on a reference model for a $5 \mathrm{MW}$ wind turbine. It is assumed herein that the presence of the OWC does not modify the mass matrix, global viscous damping and mooring.

Equivalent linear damping values are input in the WAMIT model in heave, roll and pitch to model viscous damping. The equivalent damping ratios are calibrated by comparison with an Orcaflex model of the WindFloat, discussed in a concurrent paper by Roddier et al (2011). The drag coefficients of the Orcaflex model were adjusted according to previous model tests (Cermelli and Roddier, 2005 and Cermelli et al., 2009). The dynamic characteristics of the WindFloat in heave, roll and pitch are provided in Table 2 . The equivalent damping is a fraction of critical damping:

$$
\beta=2 \sqrt{\left(M+M_{a}\right) \times C_{i}}
$$

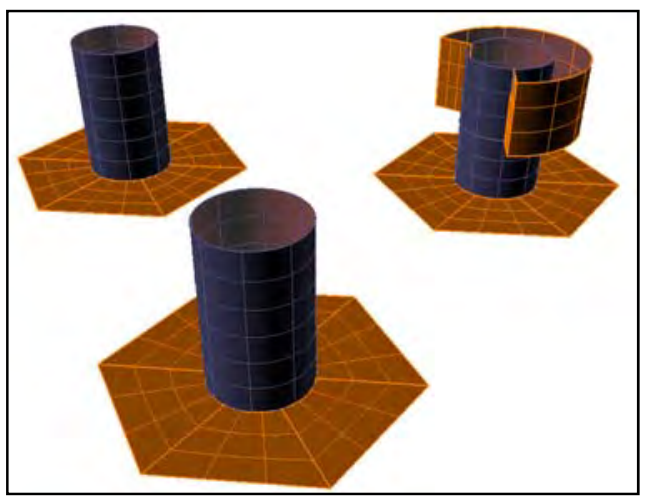

Figure 3: B-Spline Representation of WindWaveFloat Submerged Areas in Rhino3D software - Surfaces modeled as dipoles are in orange

Table 2: Dynamic Characteristics of the WindWaveFloat in Heave, Roll and Pitch

\begin{tabular}{|c|c|c|c|c|}
\hline & $\begin{array}{c}\text { Period of } \\
\text { Resonance } \\
{[\mathrm{s}]}\end{array}$ & $\begin{array}{c}\text { Added Mass } \\
{[\mathrm{kg}] \text { or Inertia }} \\
{[\mathrm{kg} \cdot \mathrm{m} 2]}\end{array}$ & $\begin{array}{c}\text { Hydrostatic } \\
\text { Stiffness }[\mathrm{N} / \mathrm{m} \\
\text { or N.m/rad] }\end{array}$ & $\begin{array}{c}\text { Critical } \\
\text { Damping } \\
{[\mathrm{kg} / \mathrm{s}]}\end{array}$ \\
\hline \hline Heave & 19.9 & $1.90 \mathrm{E}+07$ & $2.37 \mathrm{E}+06$ & $1.5 \mathrm{E}+07$ \\
\hline Roll & 43.3 & $7.76 \mathrm{E}+09$ & $2.83 \mathrm{E}+08$ & $3.9 \mathrm{E}+09$ \\
\hline Pitch & 43.2 & $7.76 \mathrm{E}+09$ & $2.83 \mathrm{E}+08$ & $3.9 \mathrm{E}+09$ \\
\hline
\end{tabular}

Similarly, the catenary mooring is modeled with an equivalent linear stiffness in WAMIT. The equivalent stiffness was taken to match the periods of resonance of the OrcaFlex catenary model in surge, sway and yaw.

Table 3: Dynamic Characteristics of the WindWaveFloat in Surge, Sway and Yaw

\begin{tabular}{|c|c|c|c|}
\hline & $\begin{array}{c}\text { Period of } \\
\text { Resonance } \\
{[\mathrm{s}]}\end{array}$ & $\begin{array}{c}\text { Added Mass }[\mathrm{kg}] \\
\text { or } \\
\text { Inertia }\left[\mathrm{kg} \cdot \mathrm{m}^{2}\right]\end{array}$ & $\begin{array}{c}\text { Equivalent mooring } \\
\text { stiffness } \\
{[\mathrm{N} / \mathrm{m} \text { or N.m/rad] }}\end{array}$ \\
\hline \hline Surge & 108.6 & $4.10 \mathrm{E}+6$ & $29,270.0$ \\
\hline Sway & 135.7 & $4.10 \mathrm{E}+6$ & $18,730.0$ \\
\hline Yaw & 71.3 & $2.76 \mathrm{E}+9$ & $4.677 \mathrm{E}+7$ \\
\hline
\end{tabular}

The internal free surface motions can be calculated using two generalized modes in WAMIT. The theoretical basis for this approach is described by Newman (1994). The first generalized mode $\zeta_{7}$ corresponds to the pumping mode and the second $\zeta_{8}$ represents the slope of the sloshing mode. In WAMIT the pumping mode is defined relatively to the vertical rigid-motion of the platform. Hence, the complex amplitude of the pumping 
mode, in the case of a free-floating platform with 0 degree incident wave and negligible roll motions, is defined as:

$\hat{\zeta}_{7}=\left(\hat{\zeta}_{\mathrm{fs}}-\hat{x}_{3}+\bar{\xi} \hat{x}_{5}\right)=1 / i \omega S \hat{\phi}$, according to Eq. 19.

The PTO is represented by an additional equivalent damping and an additional equivalent stiffness on the pumping motion of the free surface relative to the platform body, according to Eq.9. Two approaches may be taken when calculating the RAOs.

- Using WAMIT directly, a constant linear damping is applied to all frequencies.

- Using a post processing code where the equations of motion, in the frequency domain, are expressed as functions of the complex amplitudes of the displacements. Thus, by means of the hydrodynamic coefficients (added mass and damping) and the complex amplitudes of the excitation loads, the RAOs are determined for particular PTO characterizations, i.e. for specified external damping. This way the PTO damper stiffness may be adjusted to each frequency in order to get a prompt idea about the power absorption under different conditions.

Both approaches are presented in this paper. In the latter case, the PTO damping can be optimized to maximize the power take-off, as illustrated by Alves et al (.2009)

\section{EXPERIMENTAL MODELING}

Model tests are performed to validate the numerical results and calibrate the numerical model with appropriate damping values.

A model of 1:78 scale is built in acrylic. Model tests are performed at the U.C. Berkeley towing tank facility, in Richmond CA. The platform is attached to an equivalent mooring to match the global stiffness in surge, sway and yaw. To assess the effect of global radiation on the internal free surface elevation, the model can be fixed, as shown in Figure 4. Regular waves are generated with steepness between $1 / 80$ and $1 / 30$.

To estimate the performance of the $\mathrm{OWC}$, the flow rate exiting the chamber and the pressure drop between the chamber and the outside environment needs to be measured.

The flow rate is obtained by measuring the 5-degrees of freedom (surge, heave, pitch and internal free surface motions). The surge, heave and pitch motions of the platform are measured by tracking the position of two points on the WindFloat model. The tracking is performed using a video camera which is processed with tracking software WinAnalyze, by Mikromak.

The global motions of the internal free surface in the air chamber are calculated from measured free surface elevation $\zeta$ at two locations in the chamber.

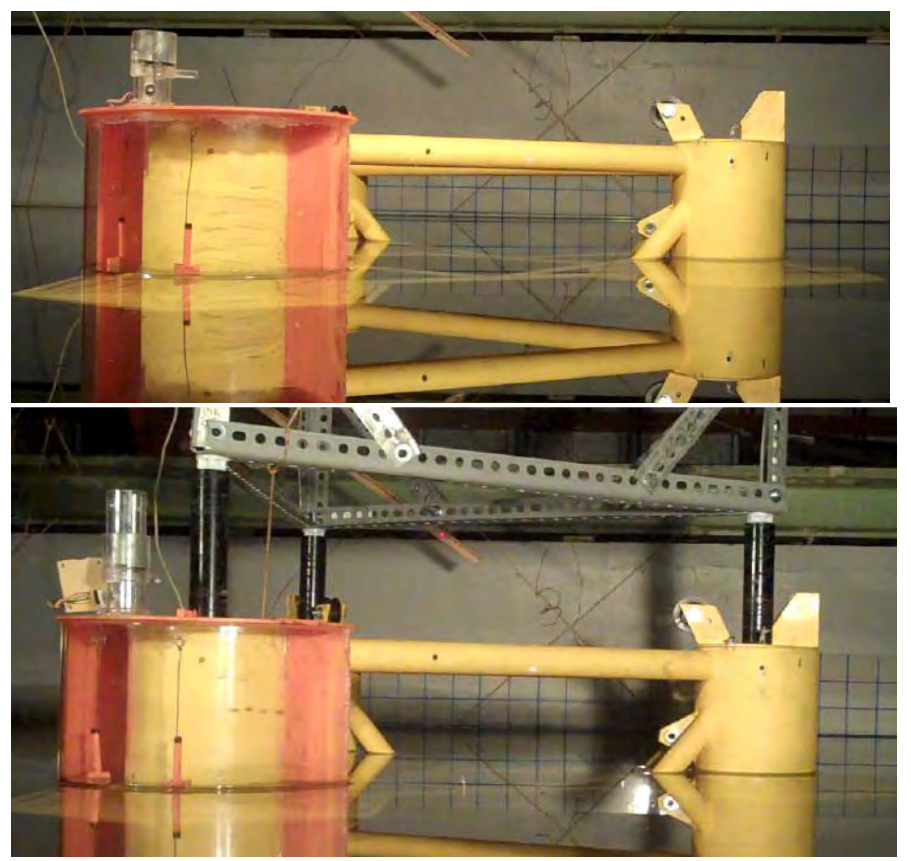

Figure 4: WindWaveFloat Model with OWC (orangetransparent shell) in the U.C. Berkeley tank - top: freefloating; bottom: fixed model

For that purpose, small floaters are located at the 180 degree and 270 degree azimuth in the chamber. They are restrained in horizontal motions and are free to move only in the vertical direction, sliding on a copper wire.

These values are obtained from the measured surface elevation $\zeta_{1}$ and $\zeta_{2}$ :

$\beta_{f s}=a \tan \left(\frac{\zeta_{2}-\zeta_{1}}{\xi_{2}-\xi_{1}}\right) \quad$ and $\quad \zeta_{f s}=\zeta_{i}-\tan \beta_{f s} \xi_{i}$

where $\xi_{\mathrm{i}}$ is the longitudinal coordinate of the point on the free surface, with respect ot the average x-position of the free surface.

The turbine is modeled with a 1 inch diameter opening at the top of the air chamber. This corresponds to a $2 \mathrm{~m}$ diameter turbine in full scale. The PTO damping is modeled with a thin porous carpet tightly fitted to obstruct this opening. This method of modelization of the turbine has been used in similar setups. It is an acceptable model of a Wells turbine, provided that a linear relation can be established between the air flow and the pressure drop through the carpet.

The pressure drop is obtained by measuring the pressure inside the chamber, upstream of the carpet. The downstream pressure, outside the chamber, is equal to atmospheric pressure. This system is bi-directional, like a Wells turbine.

For comparison purposes, all results presented herein are scaled to the full scale of the WindWaveFloat. 


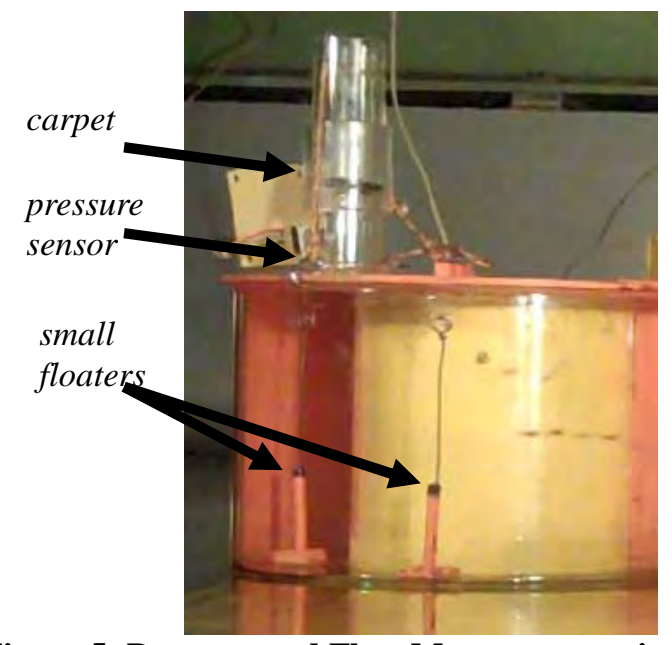

Figure 5: Pressure and Flow Measurements in the OWC of the WindWaveFloat

\section{EXPERIMENTAL VALIDATION Of OWC CHAMBER CHARACTERISTICS}

Before introducing the Power-Take-Off into the system, numerical and experimental results are compared with an open chamber.

A decay test is carried out during the model tests to verify the period of resonance of the internal free surface in the OWC air chamber. The measured natural period is $6.91 \mathrm{sec}$.

The period of resonance of the pumping and sloshing modes can be estimated numerically. Provided that cross-flow effects are minimized, it is given by:

$$
T_{i}=2 \pi \sqrt{\frac{A_{i, i}}{C_{i, i}}}
$$

Where $\mathrm{A}_{\mathrm{i}, \mathrm{i}}$ is the added mass of the OWC chamber in mode $i$ and $\mathrm{C}_{\mathrm{i}, \mathrm{i}}$ the stiffness of the chamber.

Using WAMIT calculations for the added mass and stiffness coefficient, the period of resonance of the pumping mode of the chamber is equal to $6.98 \mathrm{sec}$, as shown in Table 4 .

Response Amplitude Operators (RAOs) of internal free surface motion are obtained experimentally, with the chamber open. The air flow can exit the chamber freely through the turbine orifice since no damping is applied.

Table 4: Dynamic Characteristics of OWC Chamber

\begin{tabular}{|l|c|r|r|}
\hline \multirow{2}{*}{} & \multirow{2}{*}{ Unit } & \multicolumn{2}{|c|}{ Internal Free Surface Mode } \\
\cline { 3 - 4 } & & \multicolumn{1}{c|}{ Pumping } & \multicolumn{1}{c|}{ Sloshing } \\
\hline Added Mass & $\mathrm{kg}$ & $1.45 \mathrm{E}+06$ & $3.40 \mathrm{E}+08$ \\
\hline Stiffness & $\mathrm{kg} . \mathrm{s}-2$ & $1.18 \mathrm{E}+06$ & $4.82 \mathrm{E}+08$ \\
\hline Period & $\mathrm{s}$ & 6.98 & 5.25 \\
\hline
\end{tabular}

Experimental results are compared for two wave steepness to highlight non-linear damping effects. In the case of the steeper wave, internal free surface vertical elevation is compared when the model is free floating, with a soft spring mooring, and fixed. The resulting pumping and sloshing RAOs are plotted in Figure 6 and Figure 7 respectively.

The resonance of the internal free surface pumping mode is captured in Figure 6. The resonance of the sloshing mode is lower than 6 seconds as predicted. It was not captured due to the limitations of the model scale. Sloshing decreases rapidly when the period increases. It is small in the studied range of frequency, but it could affect the results at the period of resonance. Besides, as long as it remains small enough to avoid disturbance to the flow rate, it is not essential to the calculation of the output power. This paper focuses on the pumping mode.

Results are not significantly different between the fixed case and the free floating case. At constant steepness, the radiation potential in the 6 global degrees of freedom doesn't affect the pumping mode. However, the amplitude of the resonance varies with the steepness of the wave, as observed in the floating case. This reflects the contribution of non-linear damping from viscous effects and air flow reaction in the chamber.

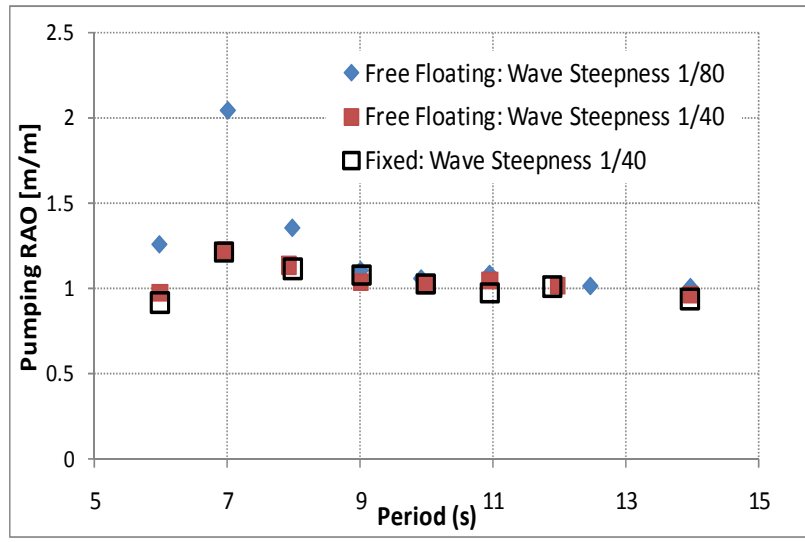

Figure 6: Amplitude of RAO of pumping mode in air chamber of OWC - Experimental results

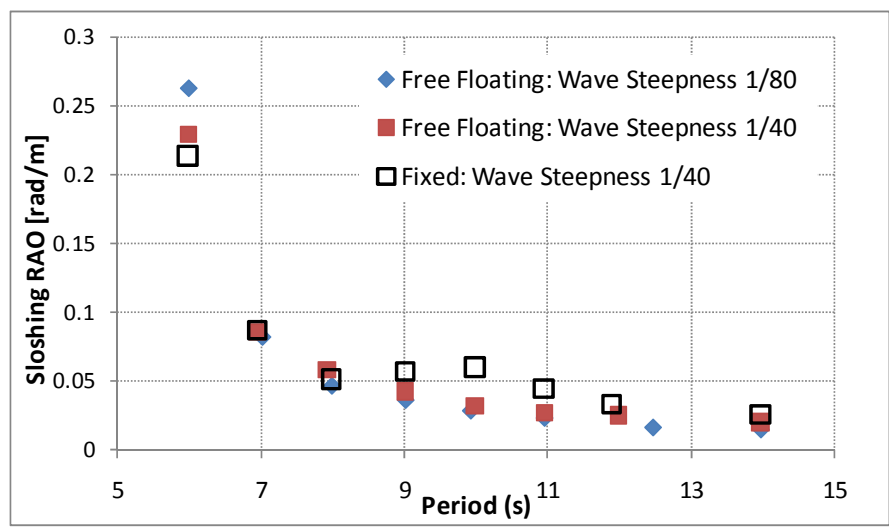

Figure 7: Amplitude of RAO of sloshing mode in air chamber of OWC - Experimental results 
The pumping RAO is compared with numerical predictions in the floating case. When wave radiation damping only is included in the computation, the amplitude of RAO is overpredicted. To quantify the amount of additional damping, numerical results are generated with an additional constant damping. It is defined as a fraction of critical damping, which in the case of pumping mode is:

$$
B_{77}=2 \sqrt{A_{77} C_{77}}
$$

Results in Figure 8 show that, at the resonance, an equivalent linear damping equal to $7 \%$ of critical damping is necessary to match the experimental results with wave steepness of $1 / 80$. With steeper waves, the equivalent damping increases to $12 \%$ of critical damping.

For future optimization of the OWC chamber, it is important to understand the distinct effect of hydrodynamic nonlinear damping due to viscous effects on the submerged hull and to aerodynamic damping generated by the losses between the air chamber and the outside environment.

The exit of the air chamber, at the top of the OWC can be modeled as an open valve. Using the Bernoulli's equation combined with the conservation of mass, and introducing a discharge coefficient $C_{d}$ to represent losses, on can express the flow rate through the open valve as:

$$
\dot{m}_{v}=\operatorname{sgn}(p) C_{d} A_{v} \sqrt{2 \rho_{o} p_{o}|p| / p_{o}}
$$

where $p$ is the relative pressure between the air chamber and the outside environment (atmospheric pressure) and $\mathrm{A}_{\mathrm{v}}$ is the cross sectional area of the valve.

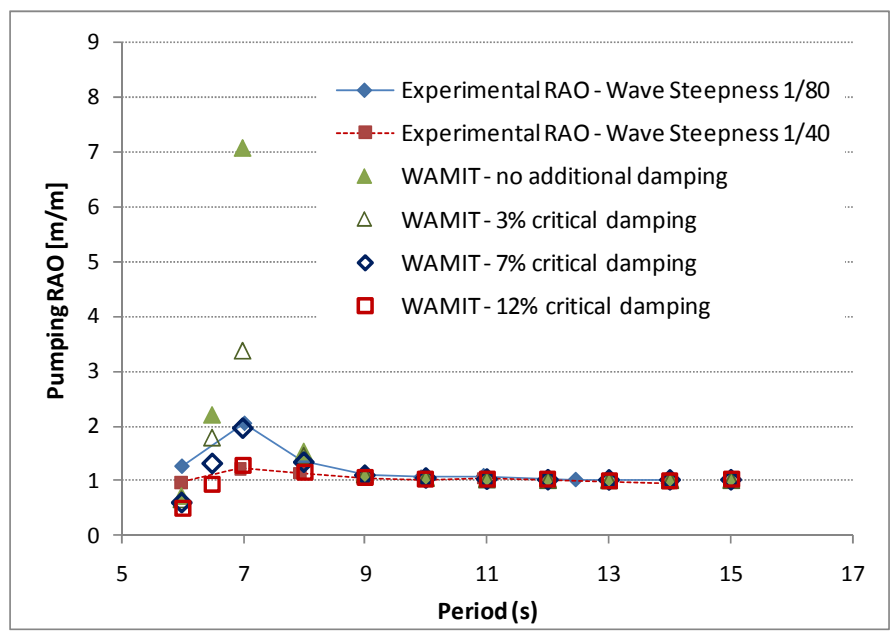

Figure 8: Comparison of WAMIT predictions and Experimental measurements of Internal Free Surface Pumping RAO in the free floating case $[\mathrm{m} / \mathrm{m}]$

Since the RMS of the measured variations of pressure inside the open chamber is smaller than $0.5 \%$ of the atmospheric pressure, the function $\operatorname{sgn}(p) \sqrt{ }|p|$ is approximated to the closest linear function with a slope equal to about 16 , which is the average slope of $\operatorname{sgn}(\mathrm{p}) \sqrt{ }|\mathrm{p}|$ for $\mathrm{p}<0.5 \% \mathrm{p}_{\mathrm{o}}$. As a result: $\dot{m}_{v}=16 C_{d} A_{v} p \sqrt{2 \rho_{o} / p_{o}}$

Combining Eq. 19 in Eq. 7, one may show that the complex amplitude of pressure associated to the pumping motion in the frequency domain is:

$\hat{p}=\frac{B_{77}}{S}\left(i \omega \hat{\zeta}_{7}\right)+\frac{K_{77}}{S}\left(-\omega^{2} \hat{\zeta}_{7}\right)$

where $\mathrm{B}_{77}$ is the equivalent linearized damping of the open valve and $\mathrm{K}_{77}$ the equivalent stiffness:

$$
B_{77}=\frac{16(S \gamma)^{2} C_{d} A_{v} \sqrt{2 \rho_{o} p_{0}^{3}}}{2\left(16 C_{d} A_{v} \gamma\right)^{2}+\rho_{o}\left(\omega V_{o}\right)^{2}}
$$

In the case of the experimental model, the exit of the air flow through the tube can be modeled as an orifice discharge with equivalent diameter ratio of 0.4 . The corresponding discharge coefficient is between 0.6 and 0.61 according to reference tables. Using $\mathrm{C}_{\mathrm{d}}=0.6$ in Eq. 22, the damping due to the air losses at the exit of the chamber is $1.13 .10^{5} \mathrm{~kg} / \mathrm{s}$, or $4.3 \%$ of critical damping. This means that at wave steepness of $1 / 40$, non-linear hydrodynamic damping contributes about two thirds of total added damping, but less than half at wave steepness $1 / 80$. However, one should be cautious when scaling these results to the full scale model due to the non-linearity of the phenomena.

\section{MODELING of POWER-TAKE-OFF}

The power take-off is modeled experimentally with a carpet. The linearity of the carpet is verified by measuring the instantaneous pressure drop inside the chamber and the flow rate, in regular waves. The RMS of the non-dimensional pressure $\Psi$ is plotted against the RMS of the flow rate coefficient $\Phi$ in Figure 9 for regular waves of constant steepness.

Here, the fluid is assumed incompressible, so that the mass flow rate is proportional to the volumetric flow rate. To attempt to model the problem numerically, the relation between pressure and flow coefficients must be linearized. At constant steepness, the relation between the pressure coefficient and the flow rate coefficient are approximated by a linear regression. It is notable that this linear regression breaks down for small pressure coefficients. This is due to the low resolution of pressure measurements in small waves. Also, the linear approximation doesn't cross the flow rate axis at the origin. The reason for this discrepancy wasn't established. It is likely due to the limitations of experimental measurements at such a small scale. 


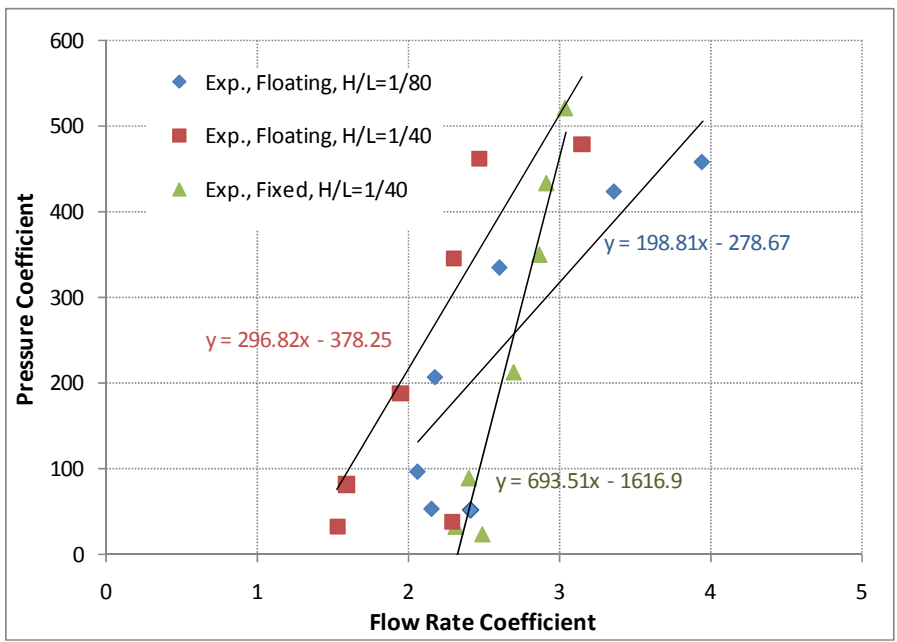

Figure 9: Experimental Relationship between Pressure Coefficient and Flow Rate Coefficient with varying Wave Steepness

These values of constant $K_{c}$ can be related to a turbine characteristic $\mathrm{K}$ between 2 and 4 for a turbine diameter $\mathrm{D}$ of 2 $\mathrm{m}$ and a rotational velocity $\mathrm{N}$ of $1000 \mathrm{rpm}$ at the resonant period, if the turbine characteristic $\mathrm{K}$ is defined as the constant of proportionality between the pressure coefficient $\Psi$ and the mass flow coefficient $\Phi$ in turbomachinery, where pressure and flow rate are non-dimensionalized with $\rho_{\mathrm{a}} \mathrm{N}^{2} \mathrm{D}^{2}$ and $\rho_{\mathrm{a}} \mathrm{ND}^{3}$ respectively. The derivation of the problem for an actual turbine is detailed by Falcão and Rodrigues (2002).

In a simplified approach, the linear coefficient $\mathrm{K}_{\mathrm{c}}$ may be used to compute the equivalent PTO damping and stiffness in Eq. 15 and 16. The resulting calculations are presented in Table 5 for the period of resonance $\mathrm{T}=7 \mathrm{sec}$. Predicted applied damping from the PTO at the period of resonance varies between 20 and $40 \%$ depending on the constant $\mathrm{K}_{\mathrm{c}}$ considered.

Table 5: Equivalent damping at the period of resonance due to modeled turbine in experiments

\begin{tabular}{|l|r|r|r|}
\hline Wave Steepness H/L & \multicolumn{1}{c|}{$1 / 80$} & \multicolumn{2}{c|}{$1 / 40$} \\
\hline \multicolumn{1}{|c|}{ Value from: } & \multicolumn{1}{c|}{ floating case } & \multicolumn{1}{c|}{ fixed case } & floating case \\
\hline K contant & 200 & 690 & 300 \\
\hline Damping w/o carpet (\% critical) & $7 \%$ & $12 \%$ & $12 \%$ \\
\hline Calculated damping from carpet (kg/s) & $9.65 \mathrm{E}+05$ & $5.07 \mathrm{E}+05$ & $8.84 \mathrm{E}+05$ \\
\hline Damping with carpet (\% critical) & $37.0 \%$ & $19.4 \%$ & $33.8 \%$ \\
\hline Total added damping (\% critical) & $44.0 \%$ & $31.4 \%$ & $45.8 \%$ \\
\hline Total applied damping (kg/s) & $1.15 \mathrm{E}+06$ & $8.20 \mathrm{E}+05$ & $1.20 \mathrm{E}+06$ \\
\hline Associated Stiffness (N/m) & $1.10 \mathrm{E}+06$ & $1.59 \mathrm{E}+06$ & $2.10 \mathrm{E}+06$ \\
\hline
\end{tabular}

Figure 10 shows the measured RAOs of the pumping mode for different wave steepness H/L. Numerical results are obtained with damping and stiffness values adjusted at each frequency, corresponding to $\mathrm{B}_{\text {РTO }}$ and $\mathrm{K}_{\text {РTO }}$. The value of constant $\mathrm{K}_{\mathrm{c}}$ is varied between 100 and 800 . An additional $7 \%$ critical damping is added to model the non-linear damping in the case of $1 / 80$ wave steepness. The center of gravity is adjusted in the numerical results to best represent that of the experimental model. The lower values of $\mathrm{K}_{\mathrm{c}}$ match the experimental measurements best. A cancellation of the pumping RAO around $\mathrm{T}=12 \mathrm{sec}$ is not well represented by the model. This may indicate that the stiffness of the PTO is over-predicted.

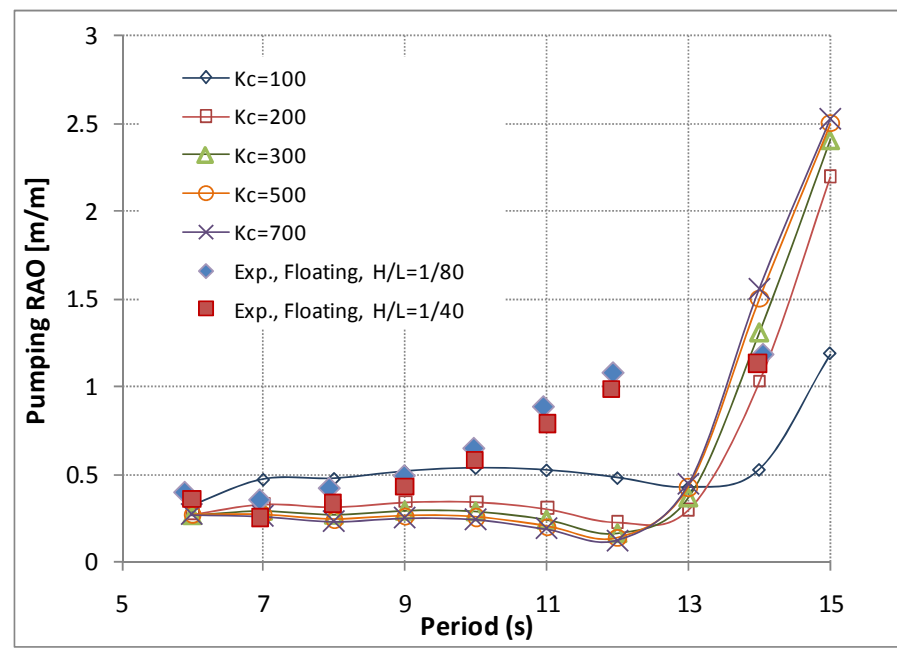

Figure 10: Pumping RAO $[\mathrm{m} / \mathrm{m}]$ in damped cases on a floating structure (with a carpet - Pumping mode in Global coordinates)

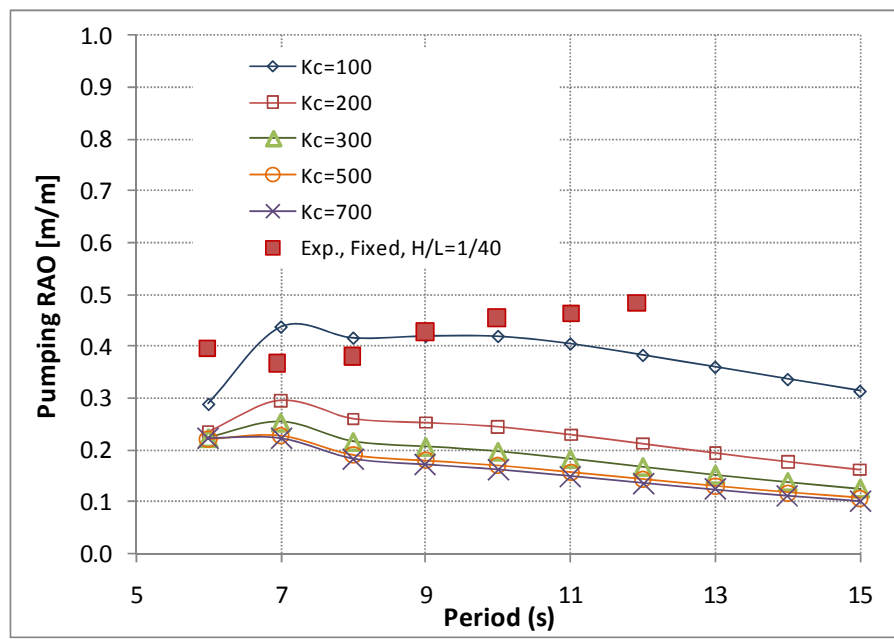

Figure 11: Pumping RAO [m/m] in damped cases (with a carpet) on the fixed structure

In experiments, at low frequencies, the pumping motion is much lower in the fixed case than in the floating case. This is predicted by linear theory. It is due to the effect of platform motion on the global free surface elevation. In Figure 11, the best match between numerical and experimental data is again observed at constant $\mathrm{K}_{\mathrm{c}}=100$.

The experimental captured width of the system, is computed according to Eq. 25 with constant $\mathrm{K}_{\mathrm{c}}=100$. The captured width is non-dimensionalized with the width of the OWC and results are compared to numerical results in Figure 12 and Figure 13. The platform motions affect the captured width at low frequencies, where the captured width of the fixed model 
decreases. The maximum measured captured width doesn't occur at the natural period of the system. That may be explained by the additional stiffness introduced by the PTO on the internal free surface pumping mode. It is notable that the maximum measured captured width doesn't occur at the natural period of the system. This is consistent with the absence of resonance at the natural period in Figure 10. Additionally, the captured width is larger in less steep waves. This reflects increased losses through non-linear effects in steep waves.

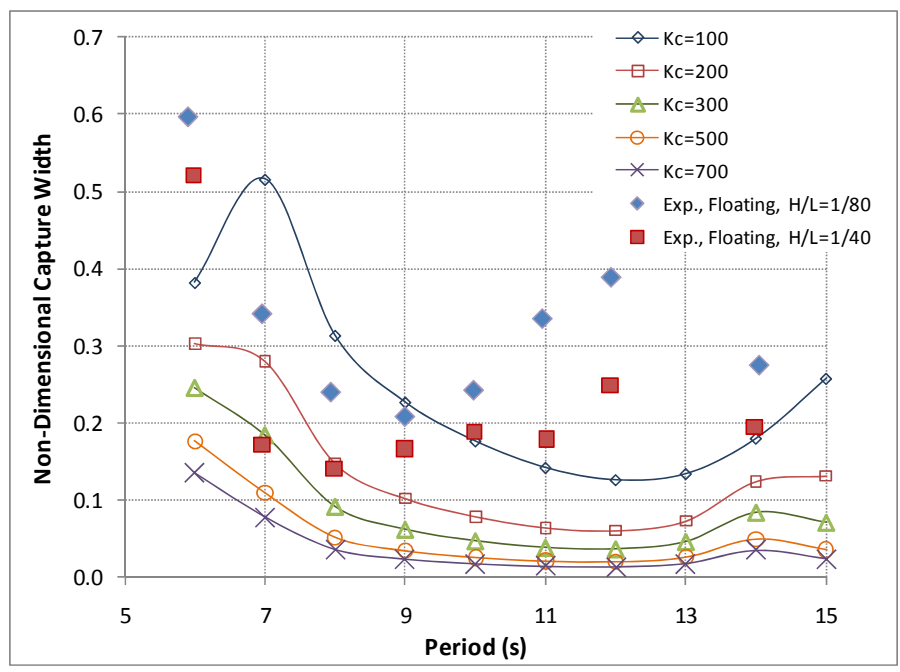

Figure 12: Comparison between Numerical (varying $K_{c}$ ) and Experimental $\left(K_{c}=100\right)$ Non-Dimensional Captured Width of the floating WindWaveFloat OWC

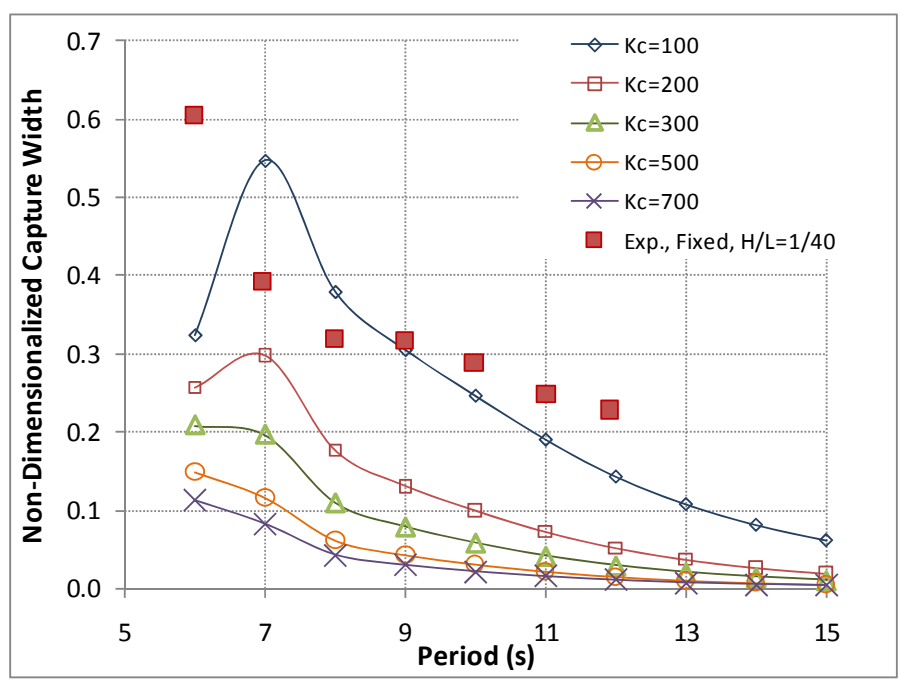

Figure 13: Comparison between Numerical (Varying $K_{c}$ ) and Experimental $\left(K_{c}=100\right)$ Non-Dimensional Captured Width of the fixed WindWaveFloat OWC

The discrepancies between numerical and experimental results in the floating cases may be linked to corresponding differences in predicted and measured roll and pitch angles of the platform.

\section{EFFECT of the OWC on PLATFORM MOTIONS}

Linear theory predicts little effect of the OWC on the WindWaveFloat motions. To validate this, platform motions were measured in experiments with and without the OWC. Results with the OWC are compared when the chamber is open and when the carpet is used to model the turbine. Results are presented in Figure 14 to Figure 16 for surge, heave and pitch.

The surge motion of the structure is well predicted by WAMIT. Results show the OWC shell and the PTO have no effect on the surge motion of the platform.

Similarly, experiments show no difference between the base case, without the OWC, and the case with the OWC shell. The PTO may introduce some effect at lower frequency, around the period of resonance of the WindWaveFloat, as can be captured at larger periods. A small difference is captured by WAMIT. However, due to non-linear effects of the water entrapment plates, WAMIT predicts a much steeper cancellation.

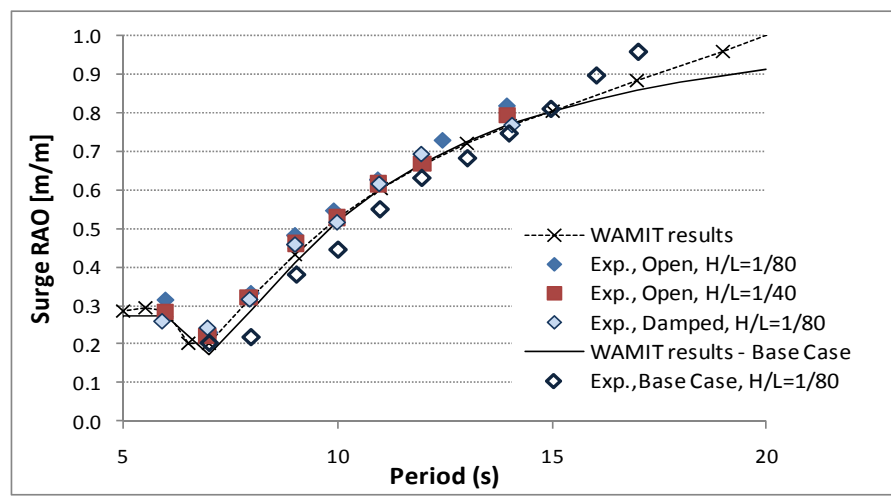

Figure 14: Comparison of Surge RAOs without the OWC (Base Case) and with the OWC

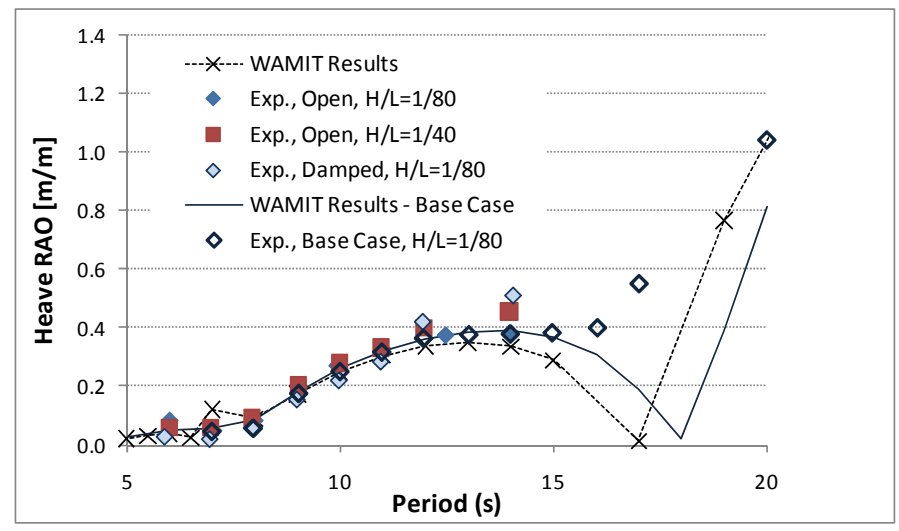

Figure 15: Comparison of Heave RAOs without the OWC (Base Case) and with the OWC

Although the experimental results are identical in pitch in the base case and with the open OWC shell, observed pitch motions are very different once the damper is added to model the turbine. This phenomenon is predicted by linear diffractionradiation theory when the equivalent PTO damping and 
stiffness are introduced. A similar effect is observed in roll, in Figure 17.

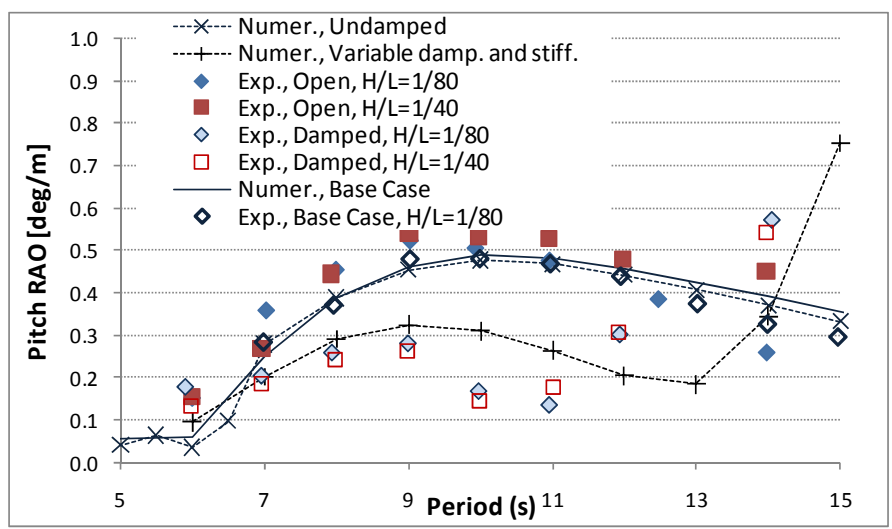

Figure 16: Comparison of Pitch RAOs without the OWC (Base Case) and with the OWC $\left(K_{c}=100\right.$ in numerical damped case)

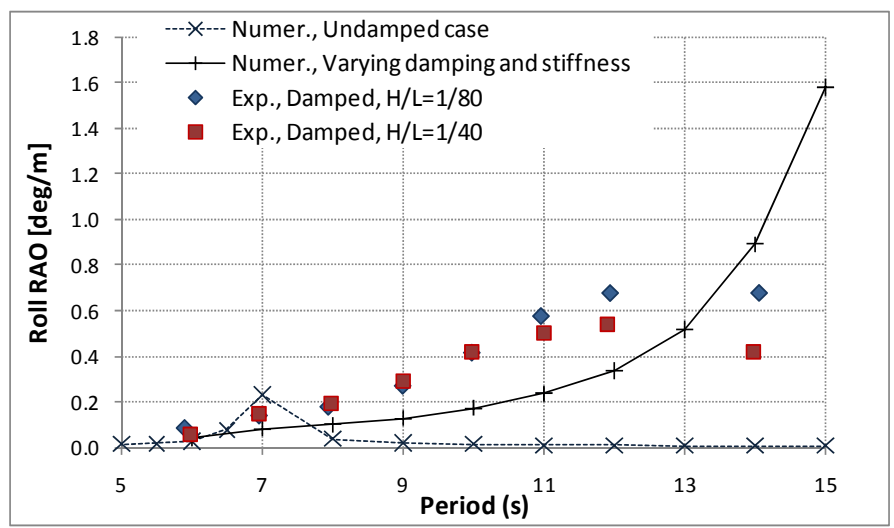

Figure 17: Comparison of Experimental Roll RAOs with a carpet damper and Numerical predictions $\left(K_{c}=100\right.$ in numerical damped case)

The roll is induced by the non-symmetrical PTO force on the platform. The damping term of the PTO force explains the reduction in amplitude of the pitch as observed in Figure 16.

\section{CONCLUSIONS}

This paper presented a frequency domain model to account for the effect of the PTO on the internal free surface of an OWC. In this framework, losses due to the discharge through the turbine as well as viscous effects are also approximated with linear damping ratios.

This model does not account for the non linearity of the Wells turbine. These non-linearities could be introduced as an efficiency ratio. In a recent analysis, Martins-Rivas (2008) has included the effect of air compressibility. It results in a loss of extracted power. Losses in the Turbine-Generator system including mechanical losses and electrical losses - were also neglected.
Model tests were carried out to calibrate the model. An equivalent PTO was obtained experimentally by modeling the Wells turbine with a linear damper. Due to limitations in the scale of the model, the calibration of the damper proved difficult. An approximation was used to obtain an equivalent model. The numerical model captures the effect of the PTO on the platform motions.

This analysis has shown that the effect of the OWC structure on platform motions is limited. However, when a PTO is introduced on one column, pitch and roll motions increase. This is a significant finding since small rotational motions are essential to the design of the wind turbine foundation. The extent of these induced rotational motions should be quantified and the impact on the tower of the wind turbine should be assessed. A more extensive analysis, across a larger range of periods should be carried out for this purpose.

The present work laid out the modeling tools for a more extensive optimization analysis, to assess the power output on the OWC and optimize its geometry and power-take-off.

\section{ACKNOWLEDGEMENTS}

This work was funded in part by a DOE financial assistance agreement (DE-EE0002652), which is duly appreciated. Nico van der Kolk, of Marine Innovation and Technology, contributed his talent and dedication. NREL's advisory role in this project and careful review of this manuscript is greatly appreciated.

\section{REFERENCES}

[1] M. Alves, T. Soulard and A.J.N.A. Sarmento "Force Reacting Principle Applied to a Heave Point Absorber Wave Energy Converter". Proc of the 19th International Offshore and Polar Eng Conf, Osaka, Japan, ISOPE, Vol I, pp 312-318, 2009.

[2] Brito-Melo, A. and Sarmento, A.J.N.A., "Numerical Modelling of OWC wave-power plants of the oscillating water column type", Advances in Boundary Elements, WIT Press, pp 25-34, 2002

[3] Brito-Melo, A., Gato L.M.C. and Sarmento A.J.N.A., "Analysis of Wells Turbine Design Parameters by Numerical Simulaiton of the OWC Performance", Ocean Engineering, Vol. 29, Issue 12, pp.1463-1477, 2002

[4] C.A. Cermelli, D.G. Roddier, "Experimental and numerical investigation of the stabilizing effects of a waterentrapment plate on a deepwater minimal floating platform", Proc. 24th International Conference on offshore Mechanics and Arctic Engineering, Halkidiki, Greece, June 2005

[5] C. Cermelli, D. Roddier, A. Aubault, WindFloat: a Floating Foundation for Offshore Wind Turbines Part II: 
Hydrodynamics, Procs. of OMAE'09, 28th International Conference on Offshore Mechanics and Arctic Engineering, Honolulu, HI, USA May 31, Jun 5, 2009

[6] Curran R., Stewart T.P. and Whittaker T.J.T., "Design Synthesis of Oscillating Water Column Wave Energy Converters: Performance Matching", Proc. Instn Mech Engrs, Vol.211, Part A, pp.489-505

[7] Falcăo, A.F.O. and Rodrigues, R.J.A., "Stochastic Modeling of OWC Wave Power Plant Performance", Applied Ocean Research, Vol.24, 2002

[8] Falnes, J., Ocean Waves and Oscillating Systems, 2002, Norwegian University of Science and Technology, Trondheim

[9] L.M.C. Gato and A.F. de O. Falcăo, "Aerodynamics of the Wells Turbine", Int. J. Mech. Sci., Vol. 30, No.6, pp.383-395. 1988

[10]Martins-rivas, $H$ and Mei, C.C., "Wave Power Extraction from an Oscillating Water Column along a Straight Coast”, Ocean engineering, Vol. 36, pp.426433, 2009

[11]Newman, J.N."Wave Effects on Deformable Bodies", Applied Ocean Research, Vol.16, pp.47-59, 1994

[12]Ragunathan S., "The Wells Air Turbine for Wave Energy Conversion”, Prog. Aerospace Sci., Vol.31, pp. 335-386, 1995

[13]D. Roddier, A. Peiffer, J. Weinstein, A. Aubault, "A Generic 5MW WindFloat for Numerical Tool Validation \& Comparison against a Generic 5MW Spar", OMAE, 2011

[14]D. Roddier, C. Cermelli, A. Aubault, A. Weinstein, "WindFloat: A Floating Foundation for Offshore Wind Turbines", Journal of Renewable and Sustainable Energy, Vol.2, Issue 3, 2010

[15] Wayman, E. N., Sclavounos, P. D., Butterfield, S., Jonkman, J., and Musial, W., "Coupled Dynamic Modeling of Floating Wind Turbine Systems," 2006 Offshore Technology Conference, 1-4 May 2006, Houston, TX

[16] R. W. Yeung, A. Peiffer, N. Tom, T. Matlak, "Design, Analysis and Evaluation of the UC-Berkeley Wave-Energy Extractor", Proc of OMAE 2010, Shanghai, China 
OMAE2011-49015

\title{
DESIGN OF A POINT ABSORBER INSIDE THE WINDFLOAT STRUCTURE
}

\author{
Antoine Peiffer \\ Marine Innovation \& Technology \\ Berkeley, CA \\ Dominique Roddier \\ Principal Power Inc \\ Berkeley, CA \\ Alexia Aubault \\ Marine Innovation \& Technology \\ Berkeley, CA
}

\begin{abstract}
This paper summarizes the modeling and testing that was performed to integrate a point-absorber type Wave-Energy Converter (WEC) within the WindFloat hull. The WindFloat is a floating structure supporting a very large $(>5 \mathrm{MW})$ wind turbine. By adding a wave-energy device to the structure, one can improve the overall economic cost of the project, since both the mooring system and power infrastructure are shared. For the device analyzed here, the modeling is first described and then the Motion Response Amplitude Operators (RAOs) are computed. From these motion responses, the theoretical mechanical power available is calculated. The power values depend on empirical coefficients that need to be confirmed through model testing in the lab. The hydrodynamic forces on each device are often dependent on the interference between the device and the hull, the mooring, and the non-linear effects which are challenging to model. Therefore, these forces are approximated using a Morrison-type formulation in the numerical models. The empirical values for drag coefficients, damping coefficients, and stiffness coefficients in this report are validated against model tests, which are also described.
\end{abstract}

\section{KEYWORDS}

Ocean Renewable Energy, Ocean Wave Energy, Offshore Wind Energy, Hybrid Systems, Floating Foundation, Point Absorbers, Power Take-off System.

\section{INTRODUCTION}

Principle Power (PPI)'s WindFloat is a floating wind turbine of multiple megawatts (MW) capacity. Because the structure is placed in an environment where both wind and wave resources are abundant, it is logical to study the effect of adding waveenergy device(s) to the WindFloat hull. The current study is framed around understanding the economic impact of adding an additional source of energy production, at a lower cost than in its stand-alone form, since mooring, electrical infrastructure and other components are being shared with the existing WindFloat.

The synergy of using an offshore wind turbine with a WEC has been explored by a few companies. Recently, the Danish company Floating Power Plant, installed a demonstrator of their Poseidon device (left, Figure 1). An elegant add-on to existing offshore wind farms is the Wave Treader, by Green Ocean Energy (right, Figure 1). The device can add up to $1 / 2 \mathrm{MW}$ of additional power to any existing monopole foundation.
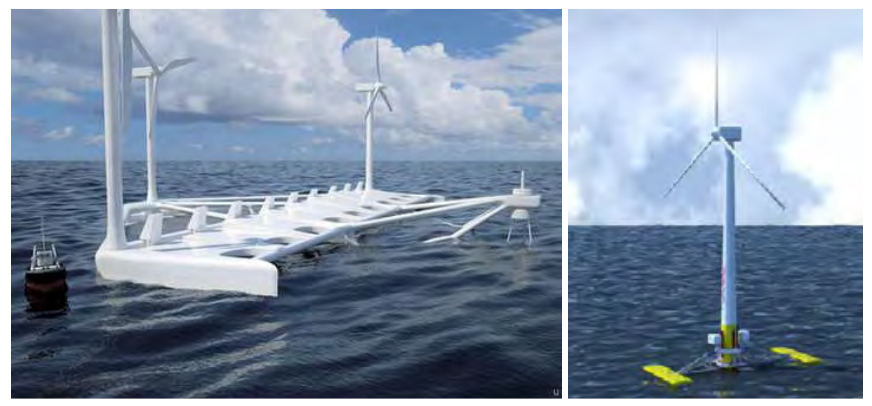

Figure 1: Existing concepts of combining Wind and Wave Energy Converters.

Economic viability of a wave energy converter depends largely on its power take-off system; an example of this can be seen in an analysis of the Pelamis [1]. It was therefore decided to study generic types of wave energy PTOs and focus on the technical integration at this stage of the work. Five types of PTOs are studied with the scope of this project. Each is integrated independently into a base case WindFloat [2], designed to support the generic NREL 5 MW wind turbine [3]. In this paper, the attention is focused on the integration of one wave-energy converter to the WindFloat platform, called the SWEDE (Spherical Wave-Energy DEvice), which is a spherical 
point absorber attached to the WindFloat columns by three springs and damping elements, mimicking the PTO (Figure 2).

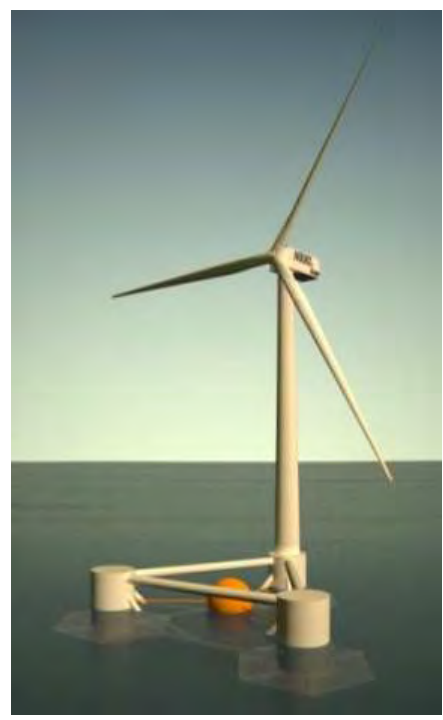

Figure 2: Single point absorber (SWEDE) integrated on the WindFloat platform.

For clarity, the SWEDE is the spherical floater without the WindFloat, and the WindWaveFloat is the set "WindFloat platform + SWEDE". The WindWaveFloat system incorporating the SWEDE is described in the first place, and the numerical modeling involving WAMIT hydrodynamic coefficients and OrcaFlex is detailed and validated based on experimental results reported here as well. In the literature, a few papers deal with the hydrodynamics of a floating sphere and its use for wave-energy applications. The results of a comparison with linear diffraction theory for the case of sphere in regular waves are shown in [4]. A discussion of the hydrodynamic and dynamic performance of one device targeted to harness wave energy by rolling motion, using a spherical floater as one of the possible shapes, is presented in [5].

The integration on the WindFloat platform of another type of wave-energy device, an oscillating water-column (OWC), is presented in [6].

\section{DESCRIPTION OF THE SYSTEM}

The WindFloat technology consists of a column-stabilized offshore platform with water-entrapment plates and an asymmetric mooring system. A wind turbine tower is positioned directly above one of the stabilizing columns. The WindFloat $5 \mathrm{MW}$ base case hull used in this study is the generic WindFloat, developed by PPI [2]. Earlier detail design work on the WindFloat is reported in [7].

The single device, called SWEDE, is a spherical point absorber installed in the center of the WindFloat platform (Figure 2). A point absorber is a floating system that absorbs energy in all directions through its movements at the water surface. A point absorber is usually designed to resonate so that its harnessed power is maximized. A spherical floater was chosen because it responds well in heave, with very little pitch motion. The SWEDE is attached to the WindFloat by three lines. Line 1 is connected to column 1, the column supporting the wind turbine. The frame of reference is presented in Figure 3 . The $z$-axis points vertically upward.

\section{NUMERICAL MODELING}

The WindWaveFloat incorporating the SWEDE is modeled with Orcaflex [8] using WAMIT [9] imported hydrodynamic coefficients. OrcaFlex is one of the leading packages for the dynamic analysis of offshore marine systems. WAMIT is a diffraction-radiation code available for analyzing wave interactions with offshore platforms and other structures or vessels.

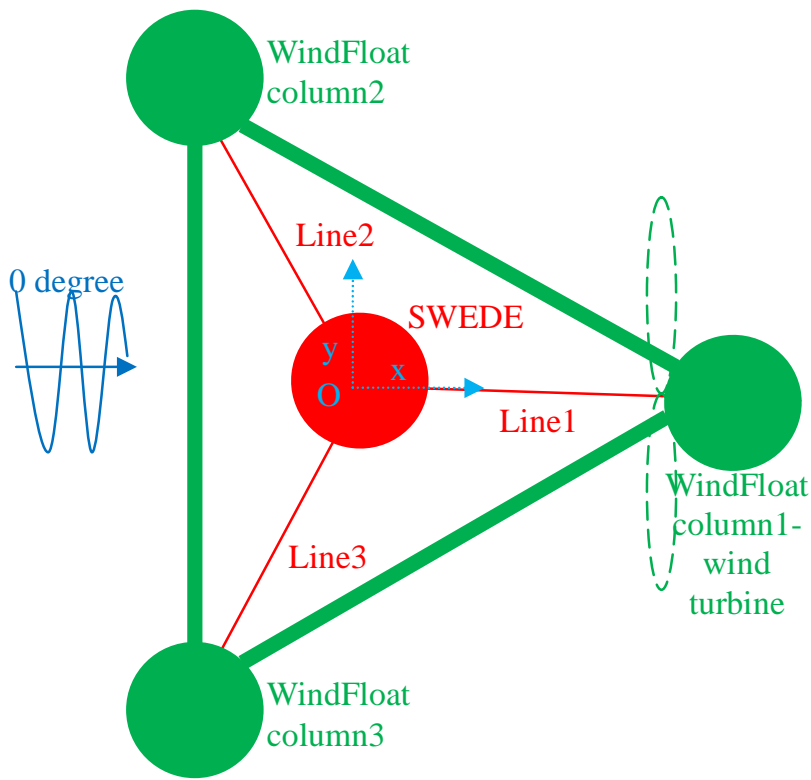

Figure 3: Configuration of the WindWaveFloat - top view

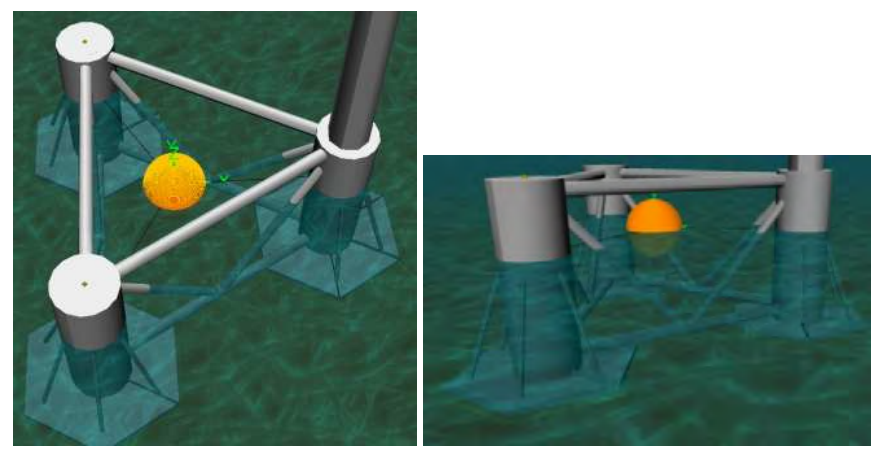

Figure 4 a, b: OrcaFlex WindWaveFloat numerical model incorporating the SWEDE - top and side view. 
The WindFloat OrcaFlex numerical model, presented and validated against experimental results as shown in [2], is used here as a basis, to which the SWEDE is added. The SWEDE is attached to the columns of the WindFloat by using three spring/damper lines representing the power take-off system (unknown at this stage of the project). Figure 4 presents the configuration of the SWEDE model. The sphere is colored in orange, and the three lines are colored in black.

\section{Modeling of the SWEDE}

In Orcaflex, the spherical floater is modeled as a series of one hundred co-axial cylinders mounted end-to-end along the local z-axis, with gradually increasing or diminishing diameters. The characteristics of the spherical floater are given in Table 1. Hydrodynamic loads on the floater are calculated using Morrison's equation. Added-mass and drag forces are applied only to those parts of the floater which are in the water at the time for which the force is calculated. The six degree-offreedom added-mass coefficients, damping coefficients, and wave-exciting loads of the floater are obtained with WAMIT using a single-body analysis. The WAMIT geometry is shown in Figure 5. We note in passing that WAMIT has the built-in assumption that the body is wall-sided above the free-surface. Details on how to perform WAMIT numerical simulations are explained in [9]. The high order method was used, due to its increase in accuracy and faster CPU computations. This recent method (Since WAMIT 6.0 release) no longer uses a quadratic mesh but the hull is modeled using Non-Uniform Rational BSpline, or NURBS. The convergence study is then performed by changing one variable in the .cfg file.

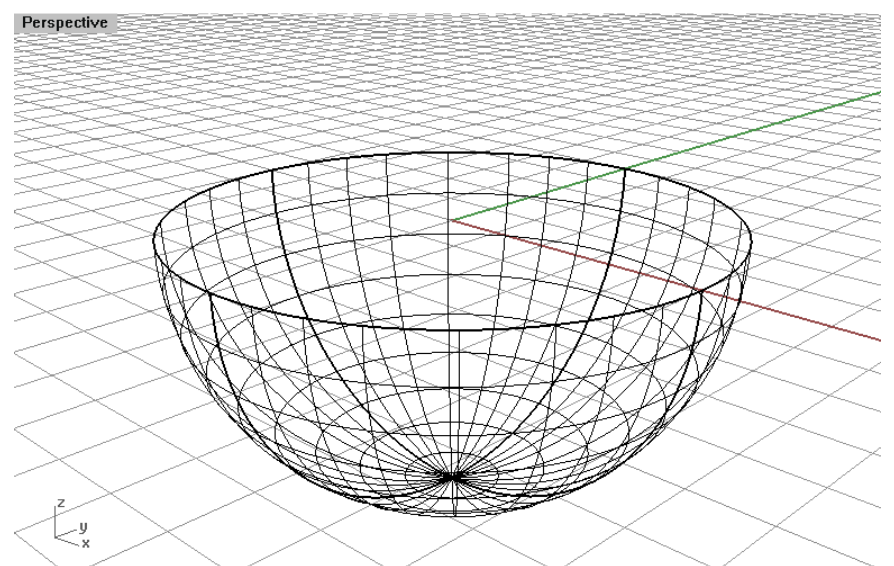

Figure 5: WAMIT geometry of the SWEDE

Table 1: Characteristics of the SWEDE

\begin{tabular}{|l|cl|}
\hline Sphere diameter D & 10.0 & $\mathrm{~m}$ \\
\hline Sphere draft d & 5.0 & $\mathrm{~m}$ \\
\hline Sphere mass/displacement $\mathbf{m}$ & 262 & tonnes \\
\hline Sphere wetted volume & 262 & $\mathrm{~m}^{3}$ \\
\hline Sphere center of mass vertical location & -3.75 & $\mathrm{~m}$ \\
\hline
\end{tabular}

\section{Modeling of the SWEDE lines}

The lines are modeled as combined springs and independent damper units. The spring can take only tension, and has a linear length-force relationship. It is pre-tensioned. The damper velocity-force relationship is also linear.

Table 2: Characteristics of the SWEDE lines (with respect to the frame of reference shown on Figure 3.

\begin{tabular}{|l|cc|}
\hline Line unstretched length & 15 & $\mathrm{~m}$ \\
\hline Line stretched length & 21.56 & $\mathrm{~m}$ \\
\hline Line1 end coordinates on the SWEDE & $(5,0,0)$ & $\mathrm{m}$ \\
\hline Line1 end coordinates on the WindFloat & $(26.56,0,0)$ & $\mathrm{m}$ \\
\hline Line2 end coordinates on the SWEDE & $(-2.5,-4.33,0)$ & $\mathrm{m}$ \\
\hline Line2 end coordinates on the WindFloat & $(-13.28,-23,0)$ & $\mathrm{m}$ \\
\hline Line3 end coordinates on the SWEDE & $(-2.5,4.33,0)$ & $\mathrm{m}$ \\
\hline Line3 end coordinates on the WindFloat & $(-13.28,23,0)$ & $\mathrm{m}$ \\
\hline Line stiffness coefficient & variable & \\
\hline Line damping coefficient & variable & \\
\hline
\end{tabular}

\section{MODEL TESTS}

A model test campaign was conducted at the UC Berkeley $200 \mathrm{ft}$ long $(61 \mathrm{~m})$ and ship model testing facility to test the validity of the numerical analysis tools. This university lab is well suited for such R\&D projects, and many wave-energy devices have been tested there in the past (see for example [10]).

A 1/78.5 scale model of the platform (Figure 6) was fabricated out of acrylic. Lead weights were placed inside the columns to adjust the center of gravity to its target position. The sphere is attached to the WindFloat columns with a system of three lines composed of wires and springs with the desired stiffness coefficient. Guiding pulleys are laid out on the column at two different levels - at the waterline and at the top of column. Two sets of springs were used to model two different SWEDE line stiffness coefficients. The friction in the pulley is supposed to be small so its damping is neglected.

Two sets of load cells: underwater load cells directly connected to the sphere, and load cells installed on top of the columns measure the instantaneous loads at two locations in the line. The difference between the load cell readings can be used to determine the experimental damping in the PTO. The wave height is measured by using a wave probe placed upstream of the model. Motions were tracked using a video tracking software, which analyses high definition mp4 videos. This relatively new software [11] (WinAnalyse) by Mikromak, works directly with the native video file from a regular HD camera, however it was found that the advanced tracking algorithms can be a bit time consuming. Additionally, when the 
motions are large, increasing the sampling rate from the typical $30 \mathrm{~Hz}$ can be beneficial.

At this point in the study, only regular sinusoidal waves of different periods and amplitudes are sent with a heading of zero degree as shown on Figure 3. All parameters used during the experimental tests and presented in this paper have been scaled up, so that the OrcaFlex numerical simulations corresponding to the experimental runs can be compared, thus the OrcaFlex numerical models can be validated at full scale.
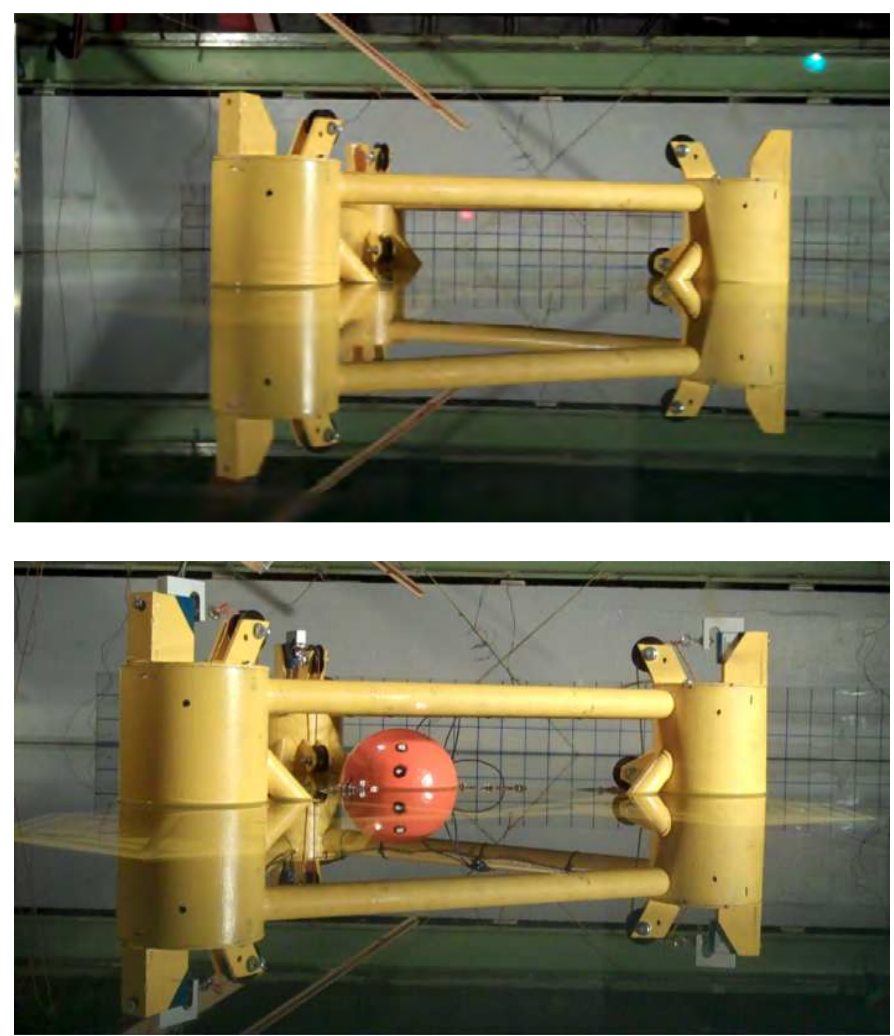

Figure 6 a,b: Pictures of the WindFloat and WindWaveFloat-SWEDE models in the UC Berkeley towing tank

\section{VALIDATION OF THE NUMERICAL MODELS}

The WindFloat platform numerical model validation is described in [2]. Only the SWEDE numerical models and the WindWaveFloat numerical models are discussed in this paper. The SWEDE damping coefficients and drag coefficients in surge, heave, and pitch are adjusted in the OrcaFlex WindWaveFloat numerical model to match the time-series of decay tests of the SWEDE alone, and the RAOs of the WindWaveFloat performed during the tank tests.

The natural periods obtained in the tank, and presented in Table 3, can be validated by theory. For example, in surge, the natural frequency $\sigma_{1}$ is defined as [12]:

$$
\sigma_{1}=\sqrt{\frac{k_{m o o r}}{m+\mu_{1}}}
$$

where $k_{\text {moor }}$ is the stiffness of the SWEDE mooring, and $\mu_{1}$ is the surge added-mass coefficient.

For three lines having a stiffness coefficient $k$ of $27 \mathrm{kN} / \mathrm{m}$ in such a parallel configuration, the global mooring stiffness $\boldsymbol{k}_{\boldsymbol{m} \text { oor }}$ in the zero-degree heading is equal to:

$$
k_{m o o r}=k+k \cdot \cos \left(60^{\circ}\right)+k \cdot \cos \left(60^{\circ}\right)=2 k
$$

Knowing the mass $m$ (262 tonnes) and the surge added-mass $\mu_{1}$ (about 166 tonnes at 17-18s) of the SWEDE, a surge natural period $T_{1}$ of $17.6 \mathrm{~s}$ is recovered. The stiffer are the lines, the shorter is the surge natural period.

In heave, the natural frequency $\sigma_{3}$ is defined as [10]:

$$
\sigma_{3}=\sqrt{\frac{k_{h y d r o}}{m+\mu_{3}}}
$$

where $k_{\text {hydro }}$ is the hydrodynamic stiffness of the SWEDE, and $\mu_{3}$ is the heave added-mass coefficient.

Again, knowing the heave added-mass coefficient at 3-4s (120 tonnes), a heave natural period $T_{3}$ of $3.7 \mathrm{~s}$ is recovered.

Table 3: SWEDE natural periods (full scale) with line stiffness of $27 \mathrm{kN} / \mathrm{m}$ (WindFloat platform fixed) based on experimental decay tests.

\begin{tabular}{|l|cc|}
\hline Surge natural period $\mathbf{T}_{\mathbf{1}}$ & 17.6 & $\mathrm{~s}$ \\
\hline Heave natural period $\mathbf{T}_{\mathbf{3}}$ & 3.7 & $\mathrm{~s}$ \\
\hline
\end{tabular}

The pitch motion is driven by either the surge of the heave motions, and peaks in pitch motion appear around peaks in surge of heave motions. This is due to the spherical shape of the SWEDE and the line configuration of the SWEDE on the WindFloat.

For completeness, the SWEDE natural periods are given in Table 4 for a SWEDE line stiffness coefficient of $140 \mathrm{kN} / \mathrm{m}$, the other tested line stiffness coefficient. The heave natural period does not change because the stiffness of the line barely affects the vertical motion of the sphere, in the small motion approximation.

Table 4: SWEDE natural periods (full scale) with line stiffness of $140 \mathrm{kN} / \mathrm{m}$ (WindFloat platform fixed) based on experimental decay tests

\begin{tabular}{|l|ll|}
\hline Surge natural period $\mathbf{T}_{\mathbf{1}}$ & 7.8 & $\mathrm{~S}$ \\
\hline Heave natural period $\mathbf{T}_{\mathbf{3}}$ & 3.7 & $\mathrm{~S}$ \\
\hline
\end{tabular}


Results including both simulations from the validated numerical models and experimental runs are presented in the next section.

\section{RESULTS}

Three types of results are presented in this section. First, the experimental RAOs of the WindFloat alone are compared with the experimental RAOs of the WindWaveFloat with the SWEDE. Second, the theoretical and experimental RAOs and force RAOs of the SWEDE are included. No damping is added to the lines at this point, because the aim is to validate the numerical models with the experimental tests, and no damping was used in the first series of experimental tests reported here. Third, once the numerical models are validated, some damping is added in the lines holding the SWEDE, in order to simulate the presence of a power take-off system. The average mechanical power generated by the SWEDE and the capture width is thus computed for different damping coefficients at the two tested line stiffness coefficients.

\section{WindFloat RAOs Comparisons: Impact of the SWEDE on the WindFloat platform}

To understand the impact of the SWEDE on the WindFloat motions, the RAOs of the WindFloat alone and the RAOs of the WindWaveFloat with the SWEDE, both measured experimentally, are compared for the same wave slope on Figure 7, for a line stiffness coefficient of $140 \mathrm{kN} / \mathrm{m}$. The wave slope $W s$ is defined as the ratio of the wave amplitude and the wavelength.

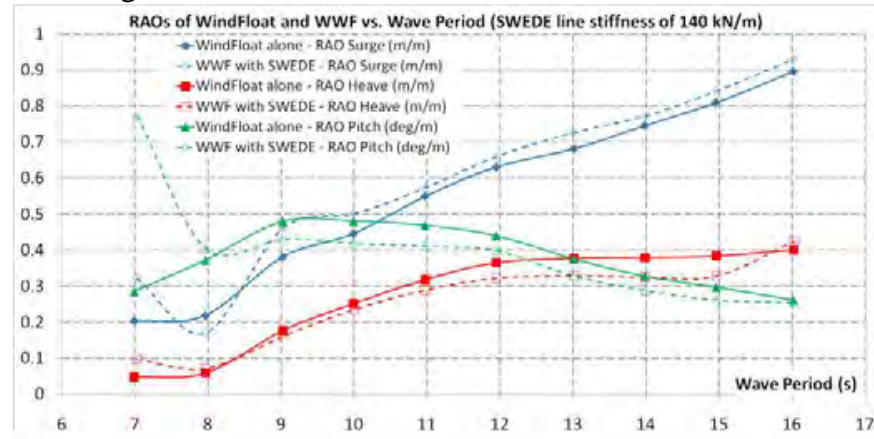

Figure 7: Comparison of the Surge, Heave, and Pitch RAOs of the WWF and the WindFloat alone. The wave slope Ws is $6 \%$.

It can be seen that the SWEDE minimally affects the motions of the WindFloat platform. Besides a slight rise of all motions after the heave period of resonance of the SWEDE (between $4 \mathrm{~s}$ and $8 \mathrm{~s}$ ), only the surge motion of the WindFloat is slightly amplified over the range of tested periods. Past the SWEDE surge period of resonance (about 8s), the WindFloat platform heave and pitch motions are even slightly reduced.

The limitation in this comparison of WindFloat RAOs vs. WindWaveFloat RAOs is that results are presented only for given SWEDE line stiffness coefficients and a zero damping coefficient. Future numerical simulations must be performed to make sure that the same conclusions can be drawn for any SWEDE line stiffness and damping coefficients, especially combinations that convert a significant amount of wave power into mechanical power.

\section{SWEDE Motion RAOs and Force RAOs}

The numerical and experimental SWEDE RAOs are presented in Figure 8 and Figure 9 for two different line stiffness coefficients, $27 \mathrm{kN} / \mathrm{m}$ and $140 \mathrm{kN} / \mathrm{m}$. There is no damping in those simulations to verify the extreme motions.

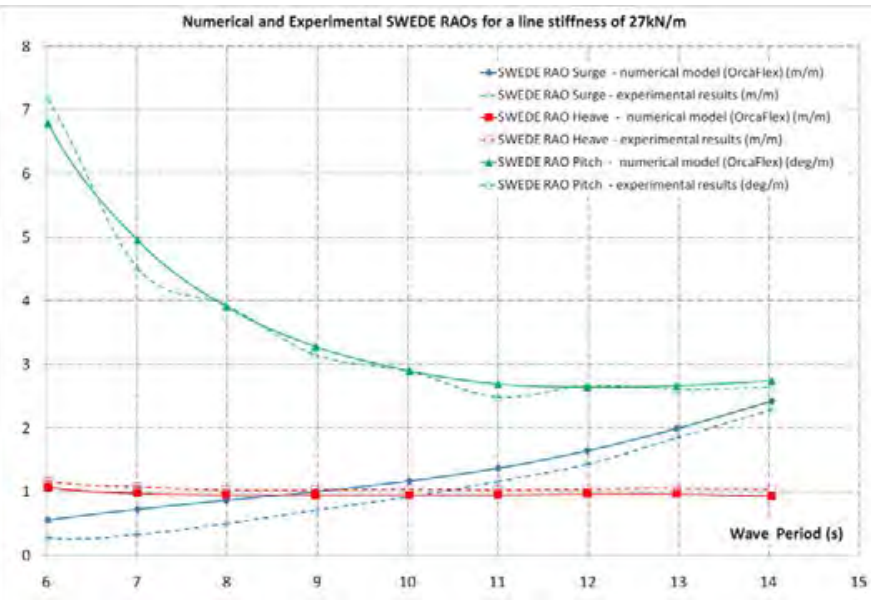

Figure 8: Comparison of numerical vs. experimental SWEDE RAOs on the WindWaveFloat for a line stiffness coefficient of $27 \mathrm{kN} / \mathrm{m}$. The wave slope $W s$ is $6 \%$.

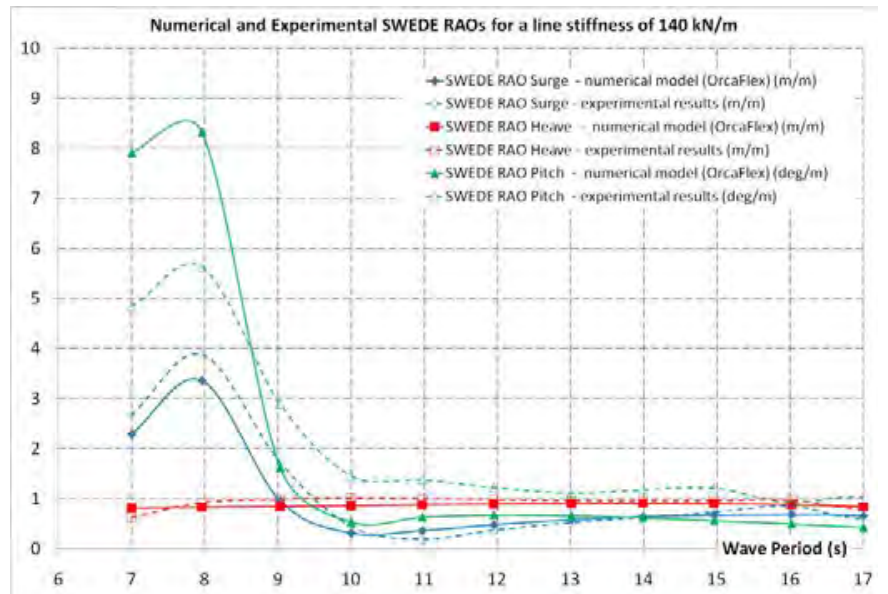

Figure 9: Comparison of numerical vs. experimental SWEDE RAOs on the WindWaveFloat for a line stiffness coefficient of $140 \mathrm{kN} / \mathrm{m}$. The wave slope $W s$ is $6 \%$.

The SWEDE presents different periods of resonance in surge, heave, and pitch for different line stiffness coefficients. Constrained by the period range allowed by the wave-maker, the RAOs presented on figure 6 show only periods out of the resonance areas in surge (17.6s), and heave-pitch (3.7s), even if the heave and pitch motions are the highest around $6 \mathrm{~s}$, and the 
surge motion keeps increasing until $14 \mathrm{~s}$. The match between numerical and experimental results is what is really sought for, and confirmed here.

The period of resonance in surge for a line stiffness coefficient of $140 \mathrm{kN} / \mathrm{m}$ clearly appears at $7.9 \mathrm{~s}$ (Figure 9). Similarly the high pitch motion is induced by the surge resonance, due to the line configuration of the SWEDE. A slight difference in peak amplitude is observed, probably due to a slight miscalculation of hydrodynamic damping. As predicted, the heave motion of the SWEDE is hardly impacted by the line stiffness coefficient, since the lines are attached to the SWEDE horizontally, at the water line level, and the major stiffness coefficient is the hydrodynamic component in this degree-of-freedom.

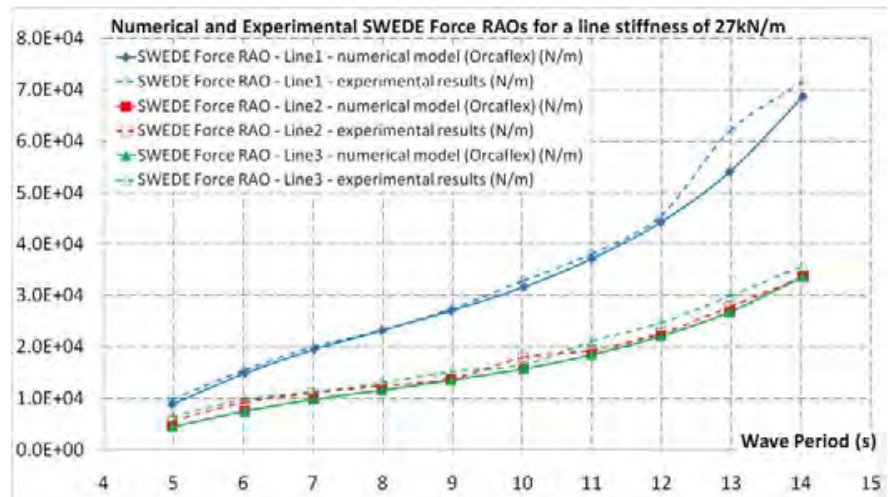

Figure 10: Comparison of theoretical vs. experimental SWEDE Force RAOs for a line stiffness coefficient of $27 \mathrm{kN} / \mathrm{m}$. The wave slope $W s$ is $6 \%$.

Similarly, the SWEDE Force RAOs, defined as the RAO of the three tensions in the line at its end connected to the spherical floater, is presented in Figure 10 for a line stiffness coefficient of $27 \mathrm{kN} / \mathrm{m}$. Figure 10 confirms the match between the numerical simulations and the experimental results, for both Motion RAOs and Force RAOs. This gives confidence in the power prediction that will be obtained numerically as it is calculated from the product of the force by the rate of change of motion.

\section{Impact of the WindFloat platform on the SWEDE RAOs and Force RAOs}

Figure 11 and Figure 12 present examples of the SWEDE Motion RAOs and Force RAOs attached to a fixed WindFloat in comparison with the SWEDE RAOs and Force RAOs in the normal WindWaveFloat configuration (freely floating WindFloat platform).

It is deduced that a freely-moving WindFloat platform slightly reduces the sphere surge and pitch motions, except around the surge resonance period $(8 \mathrm{~s})$, but reduces the significantly the tensions in the lines. A peak in Force RAO for the three lines appears at around $6 \mathrm{~s}$. The WindFloat and the sphere happen to be about 180 degree - out of phase at this period of $6 \mathrm{~s}$, thus the relative motion of the SWEDE with respect to the WindFloat is maximal, yielding the highest tensions in the SWEDE lines.

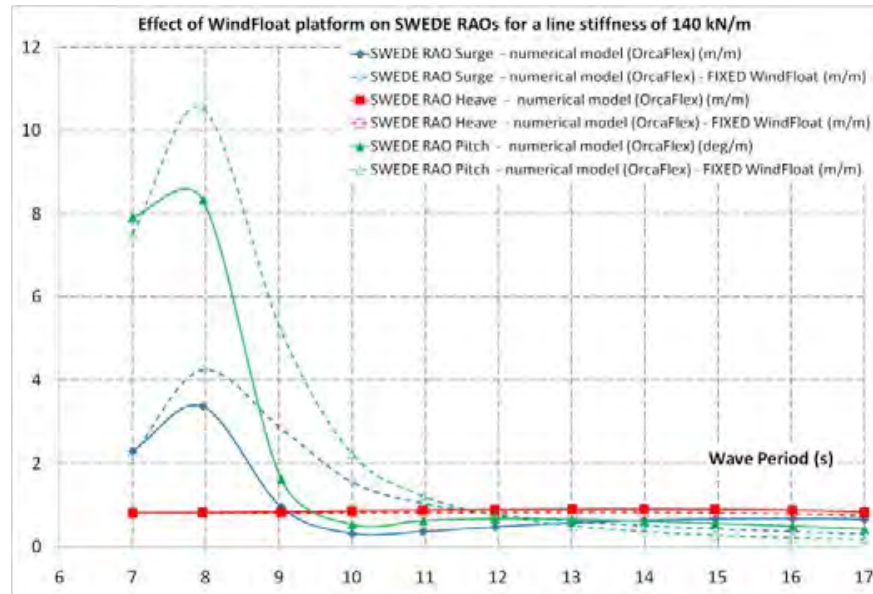

Figure 11: Comparison of numerical SWEDE Motion RAOs on a fixed WindFloat vs. freely floating WindFloat (WindWaveFloat) for a line stiffness coefficient of $140 \mathrm{kN} / \mathrm{m}$. The wave slope $W s$ is $6 \%$.

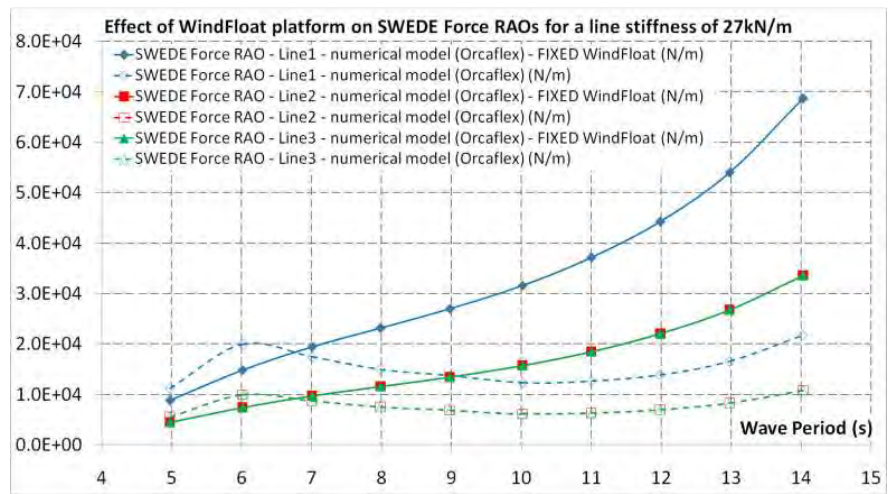

Figure 12: Comparison of numerical SWEDE Force RAOs on a fixed WindFloat vs. freely floating WindFloat (WindWaveFloat) for a line stiffness coefficient of $27 \mathrm{kN} / \mathrm{m}$. The wave slope $W s$ is $6 \%$.

\section{Power Extraction Prediction}

In this section, the mechanical power output is computed by using the numerical model in OrcaFlex validated by experimental testing. The lines holding the SWEDE to the WindFloat are modeled as a power take-off system by adding mechanical damping in the simulation.

The average mechanical energy $\mathrm{P}_{\mathrm{me}}$ received by one line is computed using the following equation:

$$
P_{m e}=\frac{1}{T} \int_{0}^{T} p(t) d t=\frac{1}{T} \int_{0}^{T} F(t) \cdot v(t) d t
$$


Where $\mathrm{p}$ is the instantaneous power, $\mathrm{F}$ is the line tension at the time $\mathrm{t}$ and, and $\mathrm{v}$ is the line velocity at the time $\mathrm{t}$. $\mathrm{F}$ and $\mathrm{v}$ are both output at the three line ends connected to the SWEDE. Figure 13 presents an example of power output times-series for the sum of the three lines.

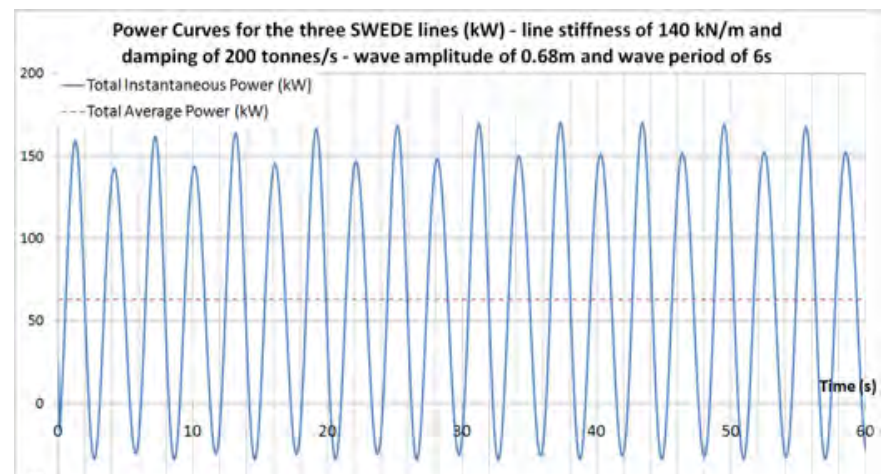

Figure 13: Example of time-series of power output for the three lines. The wave slope $W s$ is $12 \%$.

The normalized capture width $\mathrm{Cw}$ of the SWEDE, representing the ratio of the width of the wave crest that is actually harnessed and the SWEDE diameter, is computed as [10]:

$$
C_{w}=\frac{1}{D} \frac{P_{m e}}{\frac{1}{2} \rho g A^{2} V_{g}}=\frac{1}{D} \frac{P_{m e}}{\frac{1}{8 \pi} \rho g^{2} A^{2} T}
$$

where D is the diameter of the SWEDE, $\rho$ is the density of sea water, $\mathrm{A}$ is the wave amplitude, $\mathrm{T}$ is the wave period, and $V_{g}$ is the wave group velocity.

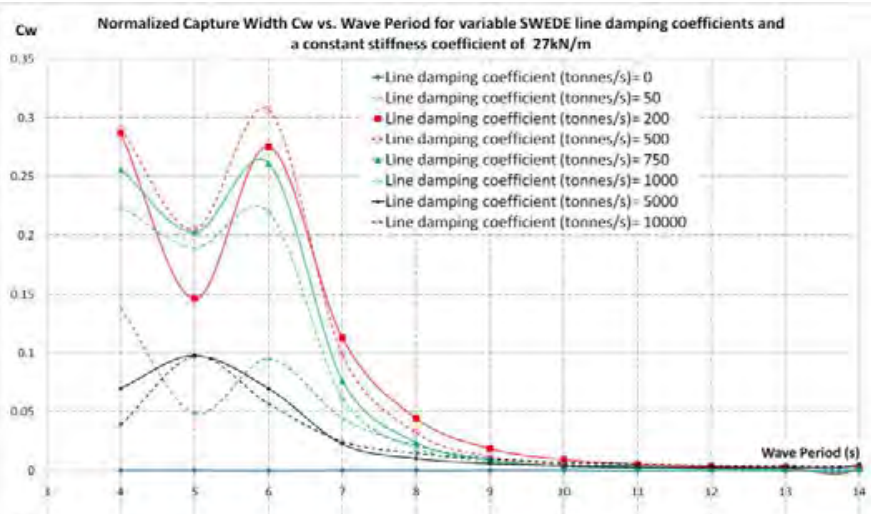

Figure 14: Normalized capture width for different SWEDE line damping coefficients and a stiffness coefficient of $27 \mathrm{kN} / \mathrm{m}$. The wave slope $W s$ is $12 \%$.

Figure 14 presents the normalized capture width of the sphere for a constant line stiffness coefficient of $27 \mathrm{kN} / \mathrm{m}$, and a varying line damping coefficient. A capture width peak of 0.3 times the diameter of the SWEDE appears at 6s for a damping coefficient of 500 tonnes/s. It means that for a regular wave of $6 \mathrm{~s}$, and the wave amplitude corresponding to the given wave slope, the wave power contained in a crest long of $3 \mathrm{~m}(0.3$ times $10 \mathrm{~m}$, the SWEDE diameter) is completely converted into mechanical power. The maximum average mechanical power, maximum combination of force and velocity (the derivative of displacement), occurs at $6 \mathrm{~s}$ for two reasons. First, as shown on Figure 12, the tension in the SWEDE lines reaches a maximum at this period. Second, the surge/pitch motion of the sphere at a period of $6 \mathrm{~s}$ is relatively high, as shown on Figure 11. The second peak in average mechanical power at a period of $4 \mathrm{~s}$ is due to the heave resonance of the SWEDE.

Figure 15 presents the normalized capture width of the sphere for a constant line stiffness coefficient of $140 \mathrm{kN} / \mathrm{m}$, and a varying line damping coefficient. There, a capture width peak of 0.6 times the diameter of the SWEDE appears at 6 s for a damping coefficient of 200 tonnes/s.

Similar values of capture widths have been retrieved for other wave-energy devices. Theoretically, considering an inviscid fluid, point absorbers can achieve capture widths independent on the size of the device, and only related to the incoming wavelength. For example, surging point absorbers, with a theoretical capture width of $\frac{\lambda}{\pi}$, where $\lambda$ is the wavelength, can achieve a capture width twice as large as heaving point absorbers [13]. For comparison, attenuators could theoretically achieve a capture width of $\frac{3 \lambda}{2 \pi}$, and the capture width of terminators would be unrelated to the wavelength, if their length is large enough [14].

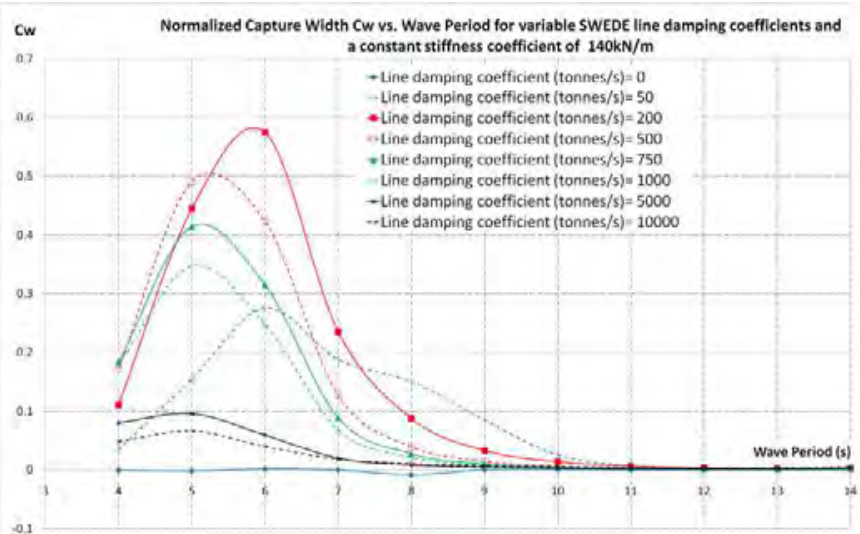

Figure 15: Normalized capture width for different SWEDE line damping coefficients and a stiffness coefficient of $140 \mathrm{kN} / \mathrm{m}$. The wave slope $W s$ is $12 \%$.

In reality, these high values of capture widths, often larger than the size of the device itself (here the diameter of the SWEDE), are rarely recovered, because of limitations due to the draft of the floater and damping issued from fluid viscosity. 
Note that the efficiency of the conversion of this mechanical power to electrical power must be taken into account in a further stage. About $60 \%$ of this mechanical power in the best conditions can be expected to be recovered at most, but that depends on the PTO system that will be used in the future.

\section{CONCLUSIONS}

In this paper, a generic 5MW WindFloat was used as the structure supporting a wave-energy device of spherical shape, the SWEDE. This WindWaveFloat system is described in detail, and numerical and experimental results of the systems are presented in regular wave conditions. The primary objective of this work was to develop the numerical tools to study the performance of this WindWaveFloat concept by validating the numerical models created in OrcaFlex (with imported hydrodynamic coefficients from WAMIT), using experimental results obtained in a wave tank at model scale. After the numerical models were validated, the wave energy extraction process, that is to say the conversion of wave energy into mechanical energy, was evaluated for several stiffness and damping coefficients in the lines of the spherical floater, modeling potential PTO characteristics. Capture widths of $6 \mathrm{~m}$ for a $10 \mathrm{~m}$-diameter spherical floater were computed and occurred at a wave period of $6 \mathrm{~s}$, where the relative motion of the sphere is maximal with respect to the WindFloat motion, leading to maximum tensions in the SWEDE lines. This favorable phenomenon enables a shift in maximum average mechanical power from the SWEDE heave resonance period ( $4 \mathrm{~s}$ here) to a slightly higher period $(6 \mathrm{~s})$, which allows the system to harness more wave power as a whole, since ocean wave periods usually range from $4 \mathrm{~s}$ to $18 \mathrm{~s}$.

More studies need to be carried out to understand the impact of irregular waves vs. regular waves on the system. Finally, the efficiency of the conversion of the harnessed mechanical energy to electrical energy is not taken into account, since the PTO has not been selected yet.

These limitations indicate that future studies need to be carried out for a better assessment of this version of the WindWaveFloat. The first piece of work should focus upon the optimization of the device, which will lead to the discovery of the optimal line stiffness and damping coefficients delivering the most power and efficiency both in regular and irregular waves. Once this optimal operational range is discovered, the selection process of an adapted PTO can start. Further engineering studies also need to be performed. The structural analysis of the system needs to be carried out, the control aspects need to be investigated, and its survivability mechanism in wave/wind storms needs to be designed. These elements will be the scope of the next paper.

\section{ACKNOWLEDGEMENT}

This work was funded in part by a DOE financial assistance agreement (DE-EE0002652), which is duly appreciated. The authors are grateful to Nico Van Der Kolk for his dedication in constructing the WindWaveFloat models and his help running the experiments. NREL's advisory role in this project and careful review of this manuscript is greatly appreciated.

\section{REFERENCES}

[1] Henderson, R., (2006), "Design, Simulation, and Testing of a Novel Hydraulic Power Take-Off System for the Pelamis Wave Energy Converter", Renewable Energy 31 (2006) 271-283

[2] Roddier D., Peiffer A., Aubault A., Weinstein J., "A Generic 5 MW Windfloat For Numerical Tool Validation \& Comparison against a Generic Spar", Proc. of OMAE 2011, Rotterdam, The Netherlands

[3] Jonkman J., Butterfield S., Musial W., and Scott G., "Definition of a 5-MW Reference Wind Turbine for offshore Development", NREL/TP-500-38060, February 2009

[4] Ballast, A., Zandbergen, P.J. ,"Fully Non-Linear Diffraction Calculations of a Floating Sphere in Regular Waves”, IWWWFB, Norway, 2005

[5] Ruiz, M. T. , "Dynamics and hydrodynamics for floating wave energy converters", Dissertação para obtenção do Grau de Mestre em Engenharia e Arquitectura Naval, Lisbon, December 2010

[6] Aubault A., Alves M., Sarmento A., Roddier D., Peiffer A. "Design of an Oscillating Water Column around the Columns of the WindFloat.", Proc. of OMAE 2011, Rotterdam, The Netherlands

[7] Roddier D., Cermelli C., Aubault A., Weinstein A. "WindFloat: A Floating Foundation for Offshore Wind Turbines", Journal of Renewable and Sustainable Energy, Vol.2, Issue 3, 2010

[8] OrcaFlex User Manual, Ulverston, UK. Last accessed in January 2011. www.orcina.com/SoftwareProducts/OrcaFlex/.../OrcaFlex. pdf

[9] WAMIT User Manual version 6.0, WAMIT Inc., MA, USA. Last accessed in January 2011. http://www.wamit.com/manual.htm

[10] Yeung R. W., Peiffer A., Tom N., Matlak T. , "Design, Analysis and Evaluation of the UC-Berkeley Wave-Energy Extractor", Proc of OMAE 2010, Shanghai, China 
[11]Frischholz R., Wittenberg T., "Computer Aided Visual Motion Analysis", Advances in Quantitative Laryngoscopy using Motion-, Image- and Signal Analysis, Erlangen, 1997

[12] Newman, J.N. "Marine Hydrodynamics", The MIT press Cambridge, Massachusetts, USA and London, England

[13]Evans D V "Some analytic results for 2D and 3D wave, energy absorbers" pp. 213-249 in "Power from Sea Waves" Ed. Count, B M, Ac. Press 1980

[14] "Marine Energy Challenge, Marine Energy Glossary", Entec, Carbon Trust Report, 2005 
Appendix D: Permitting for a Hybrid Wind/Wave Energy System in the United States 


\title{
Permitting for a Hybrid Wind/Wave Energy System in the United States
}

\begin{abstract}
This paper examines the regulatory framework for the deployment of a commercial hybrid wind/wave energy system, Principle Power Inc.'s WindWaveFloat. The paper uses as a case project a site off the coast of Oregon, in the Pacific Ocean. The various roles of US federal and state agencies are described and discussed. In addition, the paper describes the wave energy power take-off systems Principle Power, Inc. investigated as part of its study and the potential world and US markets for these products.
\end{abstract}

Principle Power, Inc.

93 So. Jackson Street, Suite 63650

Seattle, WA 98104 
Principle Power, Inc.

Permitting for a Hybrid Wind/Wave Energy System in the United States

\section{Table of Contents}

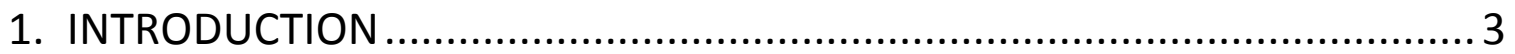

1.1 MARKETS FOR OFFSHORE WIND/WAVE AROUND THE WORLD .............................. 4

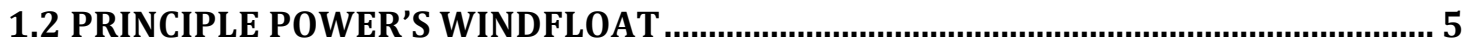

1.3 BASIS FOR WAVE ENERGY INTEGRATION

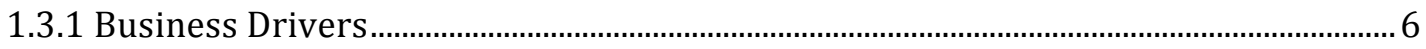

2. THE WINDWAVEFLOAT AND STUDY CONSIDERATIONS ........................ 6

- 2.1.1 Oscillating Water Column ........................................................................................ 7

- 2.1.2 Spherical Point Absorber ......................................................................................... 7

- $\quad 2.1 .3$ Oscillating Wave Surge Converter …………......................................................................... 7

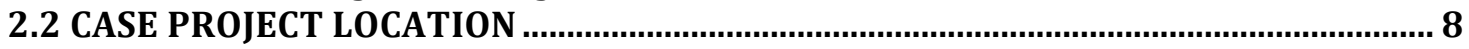

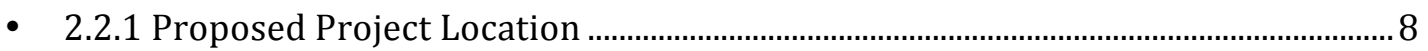

- 2.2.2 Size of Proposed Project Site ........................................................................................ 8

2.3 PRELIMINARY IDENTIFICATION OF POTENTIAL ENVIRONMENTAL ISSUES ......... 8

3. REGULATORY CONSIDERATIONS ..................................................... 9

3.1 REGULATORY STATUS: THE ROLES OF FERC AND BOEMRE.................................10

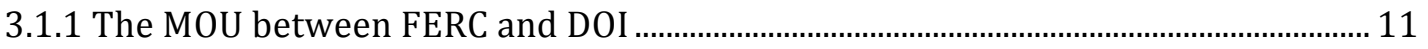

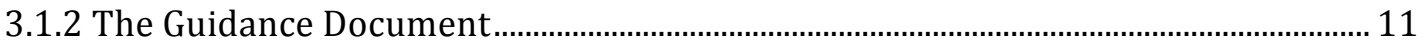

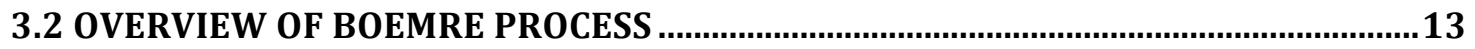

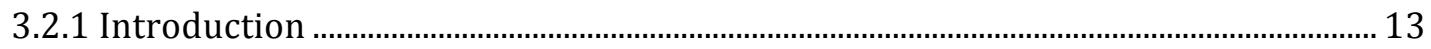

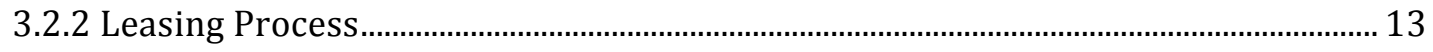

3.3 THE FERC PROCESS

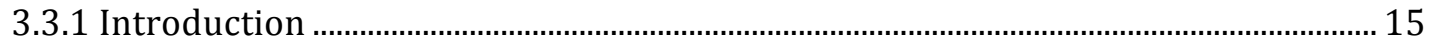

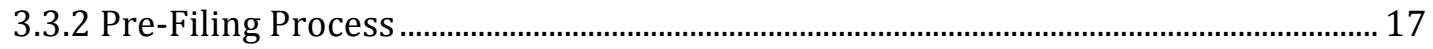

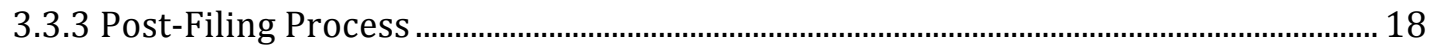

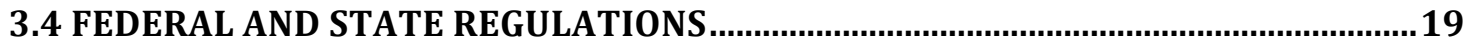

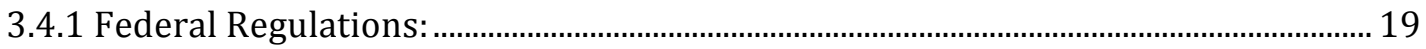

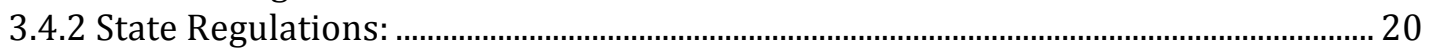

4. PROCEDURAL CONSIDERATIONS ..................................................... 20

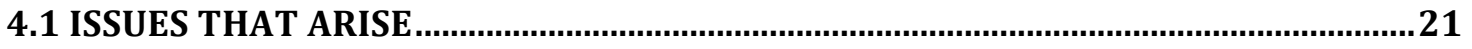

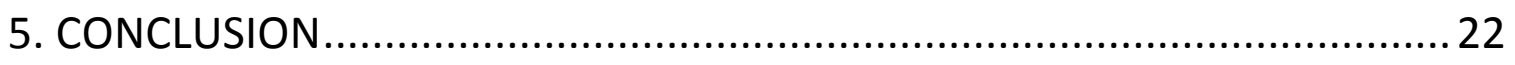

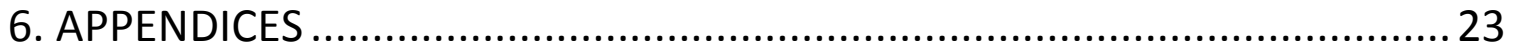

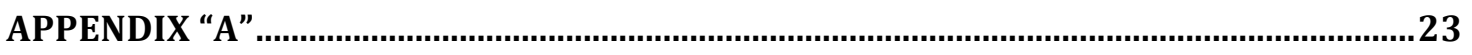

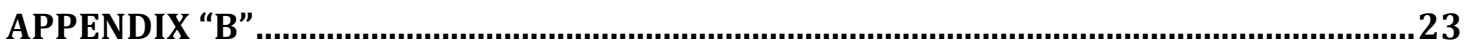

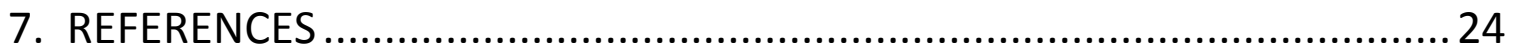


Principle Power, Inc.

\section{Permitting for a Hybrid Wind/Wave Energy System in the United States}

\section{INTRODUCTION}

Principle Power, Inc. (PPI) is the developer of the WindFloat (Figure 1), a fully integrated floating support structure for large offshore wind turbines (3.6 MW and greater). Innovative features of the WindFloat dampen wave and turbine induced motion, enabling economically efficient installation and energy extraction in water depths exceeding $50 \mathrm{~m}$.

WindFloat technology development has advanced to full-scale demonstration stage, with a unit to be deployed off the coast of Portugal in Q3 2011. This deployment is a part of a joint venture that includes the involvement of Energias de Portugal (EDP), Vestas and A. Silva Matos (ASM), a Portuguese manufacturing company, in addition to Portuguese government. The project will be grid connected and feature a Vestas V80 (2MW) offshore wind turbine.

PPI is investigating the technical and economic potential of developing a hybrid ocean energy system in which wave energy power take-off systems are integrated into the WindFloat structure. Because the WindFloat is large, stable and, in an energy project configuration, connected to the grid, this philosophy espouses economies of scale, and efficiency of energy extraction from a given location.

The environmental and ecological impacts of "Green House Gases" (GHG) and global warming are now universally acknowledged, as are their economic implications (e.g. i,ii,iii). The world's population currently consumes 15 TW of power that is predicted to increase to $30 \mathrm{TW}$ by 2050 . There is therefore a pressing need to meet the world's current and future energy demands from renewable and non-GHG emitting sources. Ocean energy resources are vast. Globally, the estimate of deep-water wave energy potential is in the range from $1 \mathrm{TW}$ to $10 \mathrm{TW}$ iv. This, coupled with similar or greater energy potential of deep water offshore wind resources, presents a compelling proposition.

Early development of large-scale offshore renewables will play an important role in achieving global goals to reduce GHG emissions and the use of depleting conventional 
energy sources. Seas and oceans cover over $70 \%$ of the earth's surface, offering huge potential for renewable energy from windv, wavevi, tidal, thermal, and osmotic sources.

After setting the context for a wind/wave hybrid energy system, and briefly examining some of the power take off systems that could be integrated into a WindFloat, this paper will provide an overview of the regulatory process presently required for the commercial deployment of a wind/wave energy device in the United States. At the time of this writing, no specialized process exists for such a project proposal

\subsection{MARKETS FOR OFFSHORE WIND/WAVE AROUND THE WORLD}

The market for offshore wind has enjoyed exponential growth over the past 10 years. This growth has occurred primarily in the North Sea, UK, Netherlands and lower Scandinavia. But to date technological and economic limitations have imposed water depth limitations on offshore wind development. The emergence of floating support structures serves to eliminate these limitations, with significant market implications. Depth and siting flexibility open sites for development that were previously unattainable in the United States, Europe and elsewhere. In addition, the wind resource in deeper water oftentimes is superior to that located on or near shore; tending to be stronger and more consistent, leading to higher capacity factor projects.

Offshore wind energy therefore has the potential to contribute significantly to the US domestic energy supply. Approximately 11,200 TWh/yr of primary energy is required to meet total current US electrical demandvii, and this figure is expected to increase by an estimated $35 \%$ by 2030 viii. In Europe, offshore wind energy has proven to be cost competitive for highly populated coastal energy markets where other energy sources are generally costly or unavailable. The coastline of the US mandates the development of new technological solutions due to a rapid drop in the continental shelf close to shore in some locations, resulting in water depths exceeding 50m: the limit of traditional bottom-fixed offshore wind installations.

There are currently no commercially viable solutions for offshore wind development in these water depths due to economic and technological limitations. As a result, only a limited area of the US (the mid-Atlantic) is suitable for offshore wind development using traditional installation techniques. Therefore, in order for the US to harness the full potential of offshore wind energy, deepwater technologies and installations must be developed and deployed. In almost all of these places, a market for wind/wave devices will exist; after all, wave energy is simply transference of winds over water, over great distances, into waves.

Several floating support structures have been publically announced and are at various stages of development. Statoil's HyWind project, a full-scale demonstration featuring a Siemens 2.3 MW offshore wind turbine has been deployed off the coast of Norway since 2009ix. Principle Power's demonstration WindFloat is planned to be deployed off the coast of Portugal in the third quarter of 2011; this will feature a Vestas 2 MW offshore wind turbine. 


\subsection{PRINCIPLE POWER'S WINDFLOAT}

The WindFloat ${ }^{x}$ is a semi-submersible structure, which uses a combination of static ballast, heave plates and an asymmetric design to achieve excellent dynamic stability performance. The performance is such that commercially available offshore wind turbines can be used following integration work on the part of the wind turbine manufacturer and Principle Power. A catenary mooring is employed to further decrease complexity and economic impact on the system. Locating the large turbine payload atop a load-bearing column maximizes structural efficiency. Mean wind induced thrust forces on the system are mitigated by a secondary closed loop active ballast system. Assembly and qualification of the system is completed quayside in a controlled environment. Offshore operations are all but eliminated as the WindFloat can be towed to and from port for installation or maintenance.

The diameter at the base of the turbine tower is nearly the same as the column diameter in order to maximize continuity of the structure, leading to minimized stress concentration in a critical area of the structure where bending moments are the highest (due to windinduced overturning moment) and large tubulars connect to the other stabilizing columns. Two other stabilizing columns are spread out as to form an equilateral triangle between the three column centers. A boat landing is installed on one or both of these columns to access the structure. The columns are interconnected with a truss structure composed of main beams connecting columns and bracings connecting main beams to columns or other main beams.

A horizontal water-entrapment plate is located at the base of each column. Stiffeners cantilevered from the bottom of the columns with bracing tying these stiffeners back to the columns support the plates. The water-entrapment plates provide additional hydrodynamic inertia to the structure due to the large amount of water displaced as the support structure moves. In addition, vortices generated at the edge of the plates generate large damping forces that further impede the support structure motion.

The general dimensions for a WindFloat featuring the NREL 5 MW reference turbine are shown in the table below. These dimensions are assumed to be equivalent to those for a WindWaveFloat.

WINDWAVEFLOAT GENERAL DIMENSIONS

\begin{tabular}{cc}
\hline \multicolumn{2}{c}{$\begin{array}{c}\text { WindWaveFloat General Dimensions } \\
\text { for the NREL 5 MW turbine }\end{array}$} \\
\hline Column diameter & $10 \mathrm{~m}$ \\
Length of water entrapment plate edge & $15 \mathrm{~m}$ \\
Column center to center & $46 \mathrm{~m}$ \\
Pontoon diameter & $2.1 \mathrm{~m}$ \\
Operating draft & $17 \mathrm{~m}$ \\
Airgap & $10 \mathrm{~m}$ \\
Bracing diameter & $1.5 \mathrm{~m}$ \\
DISPLACEMENT & 4832 \\
\hline
\end{tabular}




\subsection{BASIS FOR WAVE ENERGY INTEGRATION}

The WindWaveFloat represents a technological solution for harvesting energy from offshore renewable resources - wind, wave and tidal. It overcomes conflicting demands for stable and constrained support structure motion for wind capture, and hydrodynamic free motion for ocean energy capture, providing a floating, offshore multi-purpose renewable energy production support structure able to host wind/ocean energy converters.

\subsubsection{Business Drivers}

Ocean energy offers the benefits of significant resources without competition for scarce land, but suffers from projected high operating and installation costs. The WindWaveFloat concept offers the potential ability to increase the energy production from each floating support structure without the need for additional cabling or space. The ultimate result could lead to the reduction of levelized energy costs by as much as $25 \%$, an increase in the overall capacity factor by as much as $10 \%$ (as compared to "wind only" generation), and increased stability of the electrical power delivered to the grid.

The commercial, environmental and policy consequences of successfully integrating the conversion of wave and wind energy resources into a single device are large, but are contingent on several factors. These factors will include constructability at scale, logistics and commodity supply, and issues regarding finance and insurance. In addition, and not the least of these follow-on issues, will be the retention of favourable policies intended to bolster the renewable energy industry and the execution of laws intended to regulate the industry. Each of these factors is serious, but because current regulatory schemes largely do not contemplate hybrid energy systems, this paper will explore regulatory considerations for this type of innovative energy capture system in US waters.

\section{THE WINDWAVEFLOAT AND STUDY CONSIDERATIONS}

Deep-water (more than $50 \mathrm{~m}$ ) wave energy resources are known to be $30 \mathrm{~kW} / \mathrm{m}$ and greater [xi], offering vast potential for power generation. Offshore wave energy conversion mechanisms exploit the powerful wave regimes available in deep water and are much less dependent on the geo-morphological conditions of the coastline or on land.

There are many wave energy conversion systems in development. The main principles of wave energy conversion are based on oscillating bodies, oscillating water columns, overtopping devices, and lift-induced rotation. An important part of most power take-off (PTO) mechanisms is to have a stable fixed reference for the production of useful work. The energy is extracted through the relative forces and motions between the moving body and the fixed base (sea bottom, pile, floating frame, etc.) to which it is connected.

The WindWaveFloat design is based on the PTO solutions that are deployed and directly mounted in and/or onto the WindFloat. Using the WindFloat support structure as the basis for a new, integrated wind/wave energy conversion device for deep ocean offers many advantages as well as challenges. The use of the floating wind support structure as the reference frame for wave PTOs may result in larger motions due to the forces resulting from energy extraction, unless a proper control procedure is introduced. This control may be needed to balance the requirement of floating support structure stability against energy 
production. The final design for the integrated WWF needs to be optimized to enable the most efficient and cost effective power production between the wind turbine and the wave energy PTOs.

The WindWaveFloat is positioned with a catenary mooring, which consists of 4 mooring lines, two on column 1 (which hosts the wind tower and turbine) and one on each other column. Each line is made of 3 sections: 3 -inch chain at the fairlead, 5 -inch polyester, and 3 -inch chain to the anchor at the bottom. A clump weight is placed between the upper chain section and the polyester rope to control the tension.

PPI investigated the integration of three primary wave energy power take-off systems into the WindFloat structure. These were selected as a result of their 'popularity' among wave energy developers and the potential for appropriate integration into the WindFloat.

\section{- 2.1.1 Oscillating Water Column}

In an oscillating water column (OWC) water enters into a chamber that contains air. As waves pass, the amount of water in the chamber rises and falls like a piston, thereby sequentially compressing and decompressing the air. These positive and negatives changes in pressure result in the ability to direct the air through a bi-directional turbine coupled to an electric generator. A Wells turbine is a common choice for this type of application. See Figure 2 below.

\section{- 2.1.2 Spherical Point Absorber}

The SWEDE (Spherical Wave Energy DEvice) is a spherical point absorber situated in the centre of the structure and attached to the WindFloat columns by three springs and dampening elements. A point absorber is a floating system that absorbs energy in all directions through its movements on the waters surface. A point absorber is usually deigned to resonate so that its harnessed power is maximized. A spherical floater was selected because it responds well to heave with very little pitch motion. See Figure 3 below.

\section{- 2.1.3 Oscillating Wave Surge Converter}

In an Oscillating Wave Surge Convertor (OWSC) an arm oscillates like an inverted pendulum in response to the surrounding waves. In this case the plates are mounted on the structure main beams, outside of the water. The Oscillating Plates concept consists of three rectangular flaps hinged on the three top main beams of the WindFloat support structure. The flaps oscillate back and forth. The lower edge of each flap is attached to two lines representing the power take off system (unknown at this stage but either in the hydraulic or electrical motor category) mounted on the WindFloat support structure. See Figure 4 below. 


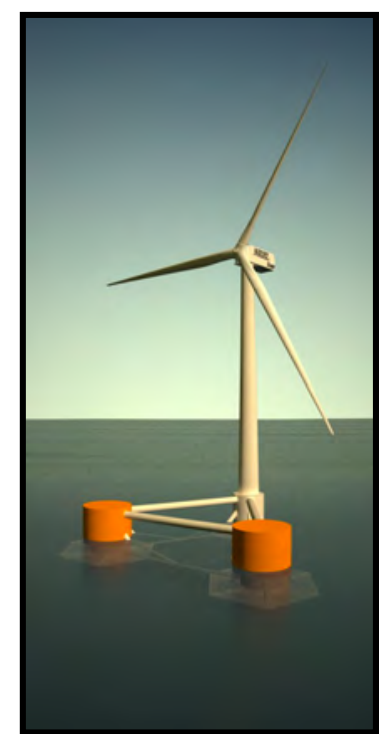

Figure 2: Oscillating Water Column

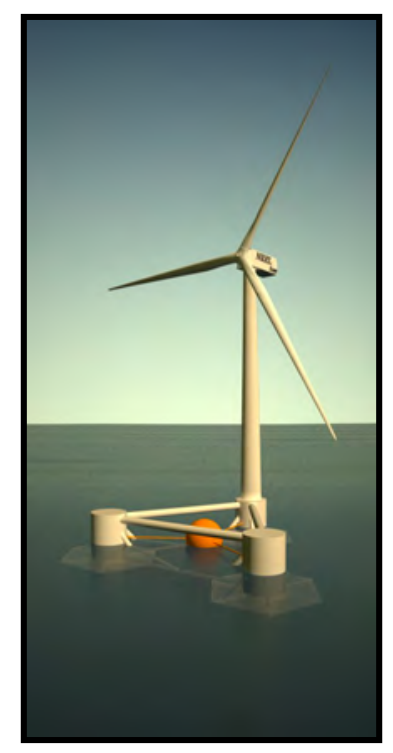

Figure 3: Spherical Point Absorber

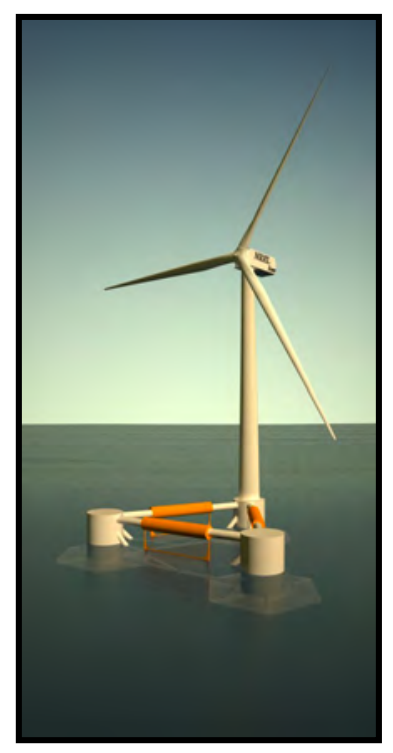

Figure 4: Oscillating Wave Surge Plates

\subsection{CASE PROJECT LOCATION}

\section{- 2.2.1 Proposed Project Location}

For this paper, PPI has proposed to generate electrical power using energy conversion of offshore wind and wave energy utilizing WindWaveFloat platforms, anchored to the ocean floor approximately nine (9) miles offshore of the coast of Netarts, Oregon. There are no ship channels or any significant restrictions to marine activities at the proposed site. The generated power would be brought to shore via submarine cable with landfall in Netarts and tie back directly into the Netarts substation $\left(45^{\circ} 27.4 \mathrm{~N}, 123^{\circ} 50.35^{\prime} \mathrm{W}\right)$.

The available wind resource has been derived from historical data collected by NOAA buoys \# 46050 and \# 46029. The wind resource potential at the location has been calculated with the use of a logarithmic wind profile law, which projects the wind energy resources $100 \mathrm{~m}$ above water. The ocean or wave roughness length used in calculations was 0.001 , typical of rough seas ${ }^{\mathrm{xii}}$.

\section{- 2.2.2 Size of Proposed Project Site}

Each WindWaveFloat installation would be spaced 5 miles apart. The surface area required for each WindFloat is 150 sq. feet. Appendix A, Figure 5 depicts the project area for the full $150 \mathrm{MW}$ installation.

\subsection{PRELIMINARY IDENTIFICATION OF POTENTIAL ENVIRONMENTAL ISSUES}

A sample list of known marine animal and plant life that exists in the area of the proposed Project is provided below. For and final project, micrositing will need to be performed in an effort to minimize any adverse impact on the environment, marine and plant life and other socio-economic considerations. 


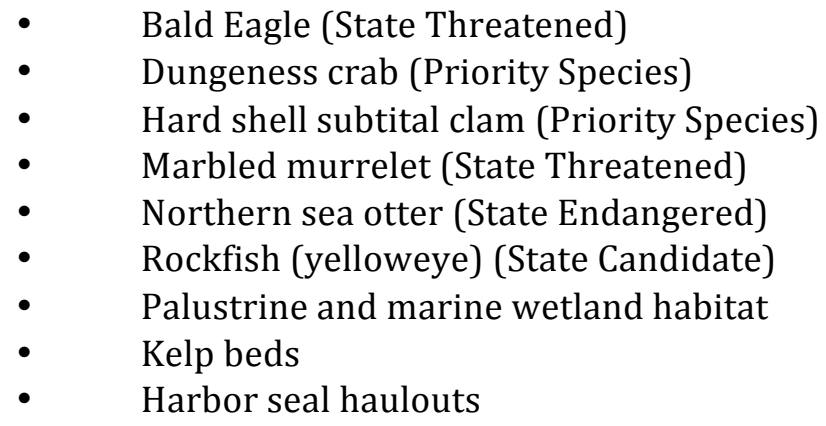

Marine mammals that may potentially be affected by development in the study area include cetaceans (Gray, Humpback, Minke, Orcas, harbor porpoise), pinnipeds (seals, sea lions), and sea otters. The location of the project relative to the migration route of gray whales would be studied, based on the historical data at the study site. Data for the current year would be included in the evaluation. Baseline information regarding the presence in seabirds and fish species in the project area would be summarized.

The environmental assessment studies performed for Cape Wind (US)xii and the Beatrice project (UK) xiv, suggest that the proposed offshore wind/wave installation would have little or no adverse environmental impact to nearby residents.

In addition, the plant would be visually unobtrusive due to its offshore location. Appendix B, Figure 6 (left) shows a view of the platform for a 6-foot tall observer standing on the beach, if he or she was 10 miles away from the target location on a very clear day. Appendix B, Figure 6 (right) shows a view of the platform 5 miles offshore on a clear day.

The proposed Project would be a clean and competitively priced energy solution that offsets the production of carbon dioxide, nuclear waste, land degradation, aviary loss, soil erosion, water pollution, eutrophication, and other environmental hazards associated with other methods for energy generation.

Toxic pollution from conventional power plants is a significant contributor to air pollution and Green House Gases on the planet. The proposed Project will help reduce carbon emissions by offsetting fossil fuel generation. Carbon dioxide (CO2) emissions will be reduced through commercialization of clean energy power plants such as the proposed Project.

\section{REGULATORY CONSIDERATIONS}

Regulatory considerations play an important role in determining the economic viability of hybrid renewable energy systems. In order to make advancements in marine renewable energy technology, devices need to be tested and the effects of their interaction with the environment need to be assessed. Substantive progress in this regard can only be achieved when full scale devices have been placed, commissioned and are operating in the locations where they will be deployed at commercial scale.

The wave energy industry is still a nascent industry and attracting and acquiring capital is a clear issue for it. Easing the path for private capital to identify opportunities for profit in the industry is an important and necessary step for it's growth. 
This necessity would appear to be outside the purview of the regulatory agencies. But the primary barrier to sourcing sufficient private market investment is risk, and uncertainty and delays in development are primary contributors to the perceived risk of investing in Ocean Energy, for both offshore wind and wave energy.

\subsection{REGULATORY STATUS: THE ROLES OF FERC AND BOEMRE}

In this case, Principle Power is proposing to obtain a permit to build and operate the WindWaveFloat system. The proposed location for the multiple devices is off the coast of Oregon on the Outer Continental Shelf (OCS). However, demonstration of a full-scale single device at this site may be the first step. The current jurisdictions give the Federal Energy Regulatory Commission (FERC) authority to grant a license for the wave component of the system. The Department of the Interior's (DOI) Bureau of Ocean Energy Management, Regulation and Enforcement (BOEMRE), has the authority to lease the land on the OCS for the entire project and permit construction and operation of the wind portion of the project. The two federal agencies have different (and time intensive) processes for approving a project.

In sum, the two agencies define their responsibilities in the following ways (in each case, the description is pulled directly from the respective agency's website):

BOEMRE: The Final Renewable Energy Framework, dated April 22 2009, “...establishes a program for BOEMRE to grant leases, easements, and rights-of-way for orderly, safe, and environmentally responsible renewable energy development activities, such as the sitting and construction of offshore wind farms on the OCS as well as other forms of renewable energy such as wave, current, and solar."

FERC: "The Commission's responsibilities include: Issuance of licenses for the construction of a new project; Issuance of licenses for the continuance of an existing project (relicensing); and Oversight of all ongoing project operations, including dam safety inspections and environmental monitoring."

To date, there has been no attempt to license a hybrid project. There have been statements made regarding the matter, however. One very important statement was made in the memorandum of understanding (MOU) between the DOI and FERC dated April 9, 2009: While this statement does not legally bind the agencies, it does indicate their knowledge of the potential conflicts involved with hybrid projects and a willingness to work together to develop a process to address these conflicts.

There have also been statements that acknowledge the need for a change in offshore wind permitting. Concerns were raised at the American Wind Energy Association (AWEA) conference on October 5, 2010, where both the Department of Energy (DOE) and DOI were in attendance. Dr. Henry Kelly, Deputy Assistant Secretary for DOE's Office of Energy Efficiency and Renewable Energy emphasized: "We need innovation in the way we go about permitting and siting and getting these activities up and running." 


\subsubsection{The MOU between FERC and DOI}

In April 2009, Department of Interior (DOI) Secretary Ken Salazar and Federal Energy Regulatory Commission (FERC) Chairman Wellinghoff signed an agreement to spur renewable energy development on the U.S. Outer Continental Shelf (OCS) - i.e., ocean waters up to 200 miles from shore, exclusive of the nearest 3 miles (or in some states, 9 miles) from shore, which are considered state waters.

"[DOI and FERC] agree to work together to the extent practicable to develop policies and regulations with respect to OCS hydrokinetic projects to carry out the purpose of this MOU. This will include, among others, processes to address hybrid (wind/hydrokinetic) projects and projects that straddle the boundaries between state waters and the OCS."xv

At first perusal, this Memorandum of Understanding, (MOU), provides clarity for the respective roles of the agencies on the OCS. Essentially, the Department of Interior's BOEMRE (then called the Minerals Management Service, or MMS) will have jurisdiction over offshore wind projects. FERC will have oversight of projects designed to generate electricity from waves, tides, and ocean currents (hydrokinetic).

But the MOU also reserves for BEOMRE the role of issuing leases, easements, and rights-ofway for hydrokinetic projects, and provides that FERC will not authorize any project that has not first obtained such authorization from MMS. This statement of shared authority while resolving, in large part, the question around jurisdictional authority also cues up additional questions regarding the rules for developing hybrid wind/hydrokinetic energy systems. Some of these questions will be discussed later in this paper.

\subsubsection{The Guidance Document}

Concurrent to the release of the MOU between FERC and MMS, a new guidance document was issued. Its stated purpose was "... to explain and provide more detail about the roles of the MMS and the FERC in authorizing the use of the OCS for hydrokinetic activities." It goes on to suggest that "... For specific guidance, prospective lessees, licensees, and other participants should rely on relevant statutes and regulations, and information and instructions provided by agency contacts, supplemented as necessary with your own source for legal advice." The full document can be found onlinexvi.

Despite its clearly constructive intent, the document's content related to hybrid projects is incomprehensive. It is reproduced in its entirety here:

\section{What is a hybrid project?}

A hybrid project, for the purpose of this guidance, is a project that includes technologies that generate electricity from more than one form of renewable energy, one of which is hydrokinetic (e.g., wind- and wave-generation under the same lease).

\section{How do I pursue a hybrid project (e.g., wind-hydrokinetic)?}

As in a single hydrokinetic lease situation, you would need to acquire a lease from MMS that covers both technologies. MMS will issue a public notice to determine whether competitive interest exists in the potential lease area, 
and may proceed with either the competitive or noncompetitive lease issuance process.

You must submit a COP to MMS for the construction and operation of the non-hydrokinetic component of your project. A FERC license (but not a COP) is required for the hydrokinetic component of your project.

\section{Can I modify my project to create a hybrid by incorporating another renewable energy technology?}

If during your lease term, you or another applicant wishes to pursue activities that are not covered by the existing lease, you or the other applicant would be required to request a separate lease, and MMS would evaluate whether or not it conflicts with existing uses prior to making a decision about whether to offer the area for additional lease(s). If joint use of an area is acceptable to both MMS and FERC, MMS will initiate the leasing process to authorize both activities (hybrid). A FERC license is required for any nonfederal hydrokinetic project on the OCS.

\section{Will MMS allow more than one type of activity on a lease?}

A lease for renewable energy activities may be held for one type of activity (e.g., wind) or for various activities (e.g., wind, wave, ocean current, etc.). MMS will determine the scope of renewable energy activities that may be allowed on a lease and issue a public notice to determine competitive interest. This notice will clearly state the scope of the lease under consideration.

If MMS determines that there is no competitive interest, MMS will follow the non-competitive lease process. If MMS determines that there is competitive interest, MMS will clearly state the scope of the lease offering early in the process and in the subsequent Proposed and Final Sale Notices. If MMS decides to limit competition to one type of activity (e.g., ocean current), MMS will not consider bids for any other type of activity, and the lease will be limited to that activity. If MMS decides to open competition to more than one type of activity or to the full set of hybrid activities, it will consider bids for the individual activities or set of activities identified, and the lease may authorize one or more of those activities. If you submit an unsolicited application, you must define your intended activities because the lease is specific to the type of project.

If you are a nonfederal applicant, you must submit to FERC a license application for the hydrokinetic component of your hybrid project and a COP to MMS for the construction and operation of the non-hydrokinetic component of your project. MMS and FERC will coordinate the interrelated reviews. 


\subsection{OVERVIEW OF BOEMRE PROCESS}

\subsubsection{Introduction}

BOEMRE issues leases and grants for renewable energy projects on the OCS. There are two types of leases and two types of grants: commercial lease, limited lease, Rights-of-Way grant, and Rights-of-Use and Easement grant. BOEMRE is required to issue all of the above competitively unless it is determined there is no competitive interest.

In January 2010, AWEA estimated an approximately 89 month (7 years, 5 months) period required to fulfill the requirements of the (at that time) MMS process ${ }^{x v i i}$. In November of that year, BOEMRE announced its Smart from the Start initiativexviii, which is intended to reduce timeframes for offshore wind development, specifically in areas of the Atlantic Coast, and in areas where specific interest in offshore wind energy development has already been registered. The Smart from the Start initiative says, in part, the following:

Implementing a comprehensive, expedited leasing framework for Atlantic wind by: (i) identifying "wind energy areas" (WEAs) along the OCS that appear particularly well- suited for development; (ii) over the next six months, organizing, financing and implementing the gathering of information from key agencies regarding the environmental and geophysical attributes and other uses of these WEAs; and (iii) assembling that information in a publicly available format that potential investors and applicants can access and BOEMRE can use in evaluating lease sales in the WEAs.

Simplifying the approval process for individual proposed projects and eliminating unnecessary regulatory requirements, including dispensing with a requirement to issue a duplicative second notice where there is no competitive interest in a lease area.

Moving aggressively, on a parallel (but separate) track, to process applications to build offshore transmission line(s). The assessment of WEAs should assist in the siting and environmental reviews associated with potential offshore transmission line(s).

Because in this case PPI would intend to use the lease for commercial power generation and since it appears unlikely there will be competitive interest in the intended project location, the following details apply to a non-competitive commercial lease. The regulations defining the process can be found in the Code of Federal Regulations Title 30 Part 285 on the website: ecfr.gpoaccess.gov.

\subsubsection{Leasing Process}

Principle Power would not be responding to a BOEMRE issued Request for Interest (RFI) but instead submitting an unsolicited request for a lease. This request must include the area of interest on the OCS, a description of the proposed project, a general schedule of activities, any available environment or resource data, a statement that the project agrees with state and local energy planning objectives, a complete set of qualification documents, and an acquisition fee of $\$ 0.25$ per acre. The qualification documents should demonstrate technical 
and financial capability and show that the company/developer is organized under the laws of a state of the United States. Any privileged or confidential information will be withheld from public disclosure.

According to the process BOEMRE will then issue public notice of an RFI in the area that the unsolicited request involves. A determination of competitive interest will be made based on the comments received in response to the RFI. The comment period following the RFI is typically 60 days but can vary. BOEMRE will then issue a notice of non-competitive determination. After this notice the lessee must submit the Site Assessment Plan (SAP) within 60 days. The approval of the SAP and the issuance of the lease will occur at the same time. Once the SAP is approved, the site assessment term of the lease will begin. The site assessment term of a lease is 5 years.

The SAP describes the activities planned to characterize the commercial lease. These activities may include installation of meteorological towers or buoys or technology testing. Physical characterization surveys such as geophysical, geological, and hazard surveys as well as baseline environmental surveys such as biological and archaeological surveys may be conducted in order to prepare the SAP. These studies can be permitted under the U.S. Army Corps of Engineers' (ACOE) Nationwide Permit program and no longer need BOEMRE approval. The data acquired in these surveys must be included in the SAP. The applicant must also prepare a consistency certification to submit with the SAP. See 30 CFR 285.605618 for the regulations governing the SAP.

It is permitted to submit both the SAP and Construction and Operations Plan (COP) at the same time, which can add efficiency to the process. If the COP is submitted early it must contain sufficient information for BOEMRE to complete the technical and environmental reviews. Otherwise, the COP must be submitted at least 6 months before the end of the site assessment term. Construction of the project may not begin until the COP has been approved. If a join SAP/COP is submitted and approved, the operations term begins 5 years after it is approved or when fabrication and installation begin, whichever comes first. If the COP is submitted separately, the operations term begins when the COP is approved. The operations term of the lease is 25 years.

The COP describes construction, operation, and conceptual decommissioning plans for the wind portion of the project. The plans must include those for the project easement for the transmission cable. The plans must cover all proposed activities in the project area including maintenance activities. The COP must include information such as the location and requirements (land, labor, material, energy) of the operation and facilities. See 30 CFR 285.620-638 for the regulations governing the COP.

Both the SAP and COP are required to show that the activities conducted obey all laws and regulations, are safe, do not unreasonably interfere with other uses of the OCS, do not cause undue harm, use the best available and safest technology, use best management practices, and use properly trained personnel. Both plans must comply with the National Environmental Policy Act (NEPA) and the Coastal Zone Management Act (CZMA) in addition to the numerous other regulations described below. The NEPA documents (Environmental Assessment - EA or Environmental Impact Statement - EIS) will be prepared by BOEMRE. Which document is prepared and how long the NEPA analysis takes depends on the complexity of the project. 
An EA is a document used to determine if a proposed activity will significantly affect the environment. If there are no such effects, BOEMRE will issue a finding of no significant impact (FONSI) which may include mitigation measures necessary to avoid such impacts. If an EA is used the NEPA analysis is about a 12 month process.

An EIS is prepared if an EA finds significant environmental consequences. The EIS is a more detailed evaluation than the EA. The public and other federal agencies (besides the preparer) may provide input in the preparation process for the EIS. A draft EIS is issued followed by a comment period before a final document is prepared. If an EIS is used the NEPA analysis is about a 24 month process.

After the final NEPA document is issued for both the SAP and COP (or joint SAP/COP) BOEMRE can approve the lessee's plans and the lease is issued with the terms indicated above.

\subsection{THE FERC PROCESS}

\subsubsection{Introduction}

There are three possible processes for obtaining a hydrokinetic license from the Federal Energy Regulatory Commission. The three possible processes are the Integrated Licensing Process (ILP), Traditional Licensing Process (TLP), and Alternative Licensing Process (ALP). The ILP is the default process used and both the TLP and ALP require pre-approval from FERC. Flow charts representing the FERC processes can be found on the FERC websitexix.

\begin{tabular}{|c|c|c|c|}
\hline & $\begin{array}{l}\text { Integrated Licensing Process } \\
\text { (ILP) }\end{array}$ & $\begin{array}{c}\text { Traditional } \\
\text { Licensing Process } \\
\text { (TLP) }\end{array}$ & $\begin{array}{l}\text { Alternative Licensing } \\
\text { Process (ALP) }\end{array}$ \\
\hline $\begin{array}{l}\text { Consultation } \\
\text { w/ Resource } \\
\text { Agencies and } \\
\text { Indian Tribes }\end{array}$ & - Integrated & - Paper-driven & - Collaborative \\
\hline $\begin{array}{l}\text { FERC Staff } \\
\text { Involvement }\end{array}$ & $\begin{array}{l}\text { - Pre-filing [beginning at filing of } \\
\text { Notice of Intent (NOI)] }\end{array}$ & $\begin{array}{l}\text { - Post filing (after } \\
\text { the application has } \\
\text { been filed) }\end{array}$ & $\begin{array}{l}\text { - Pre-filing (beginning at filing } \\
\text { the NOI) }\end{array}$ \\
\hline & - Early and throughout process & $\begin{array}{l}\text { - Available for } \\
\text { education and } \\
\text { guidance }\end{array}$ & $\begin{array}{l}\text { - Early involvement for } \\
\text { National Environmental Policy } \\
\text { Act (NEPA) scoping as } \\
\text { requested }\end{array}$ \\
\hline Deadlines & $\begin{array}{l}\text { - Defined deadlines for all } \\
\text { participants (including FERC) } \\
\text { throughout the process }\end{array}$ & $\begin{array}{l}\text { - Pre-filing: some } \\
\text { deadlines for } \\
\text { participants }\end{array}$ & $\begin{array}{l}\text { - Pre-filing: deadlines defined } \\
\text { by collaborative group }\end{array}$ \\
\hline & & $\begin{array}{l}\text { - Post-filing: defined } \\
\text { deadlines for } \\
\text { participants }\end{array}$ & $\begin{array}{l}\text { - Post-filing: defined deadlines } \\
\text { for participants }\end{array}$ \\
\hline $\begin{array}{l}\text { Study Plan } \\
\text { Development }\end{array}$ & $\begin{array}{l}\text { - Developed through study plan } \\
\text { meetings with all stakeholders }\end{array}$ & $\begin{array}{l}\text { - Developed by } \\
\text { applicant based on }\end{array}$ & $\begin{array}{l}\text { - Developed by collaborative } \\
\text { group - FERC staff assist as }\end{array}$ \\
\hline
\end{tabular}




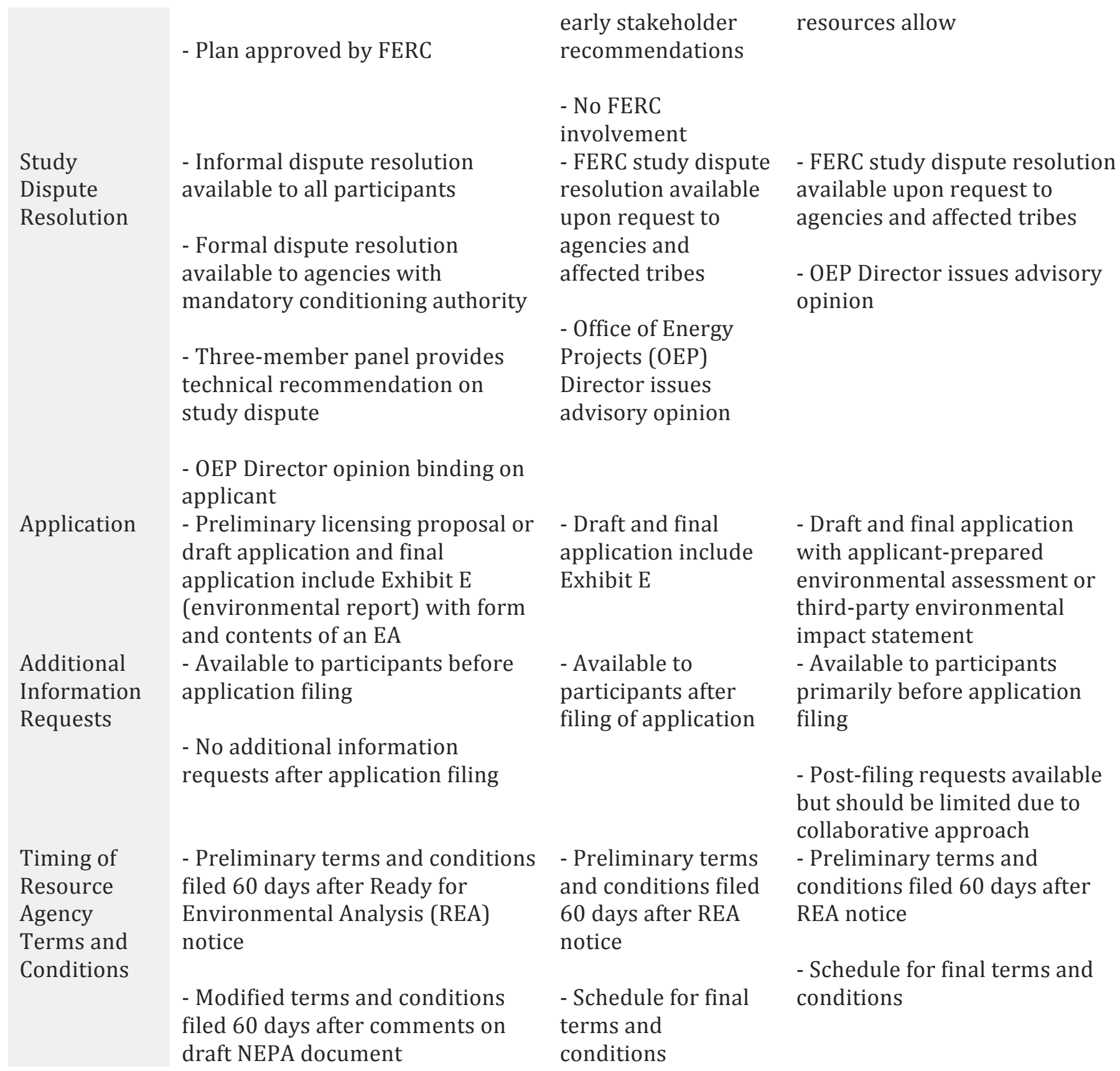

The following detail is for the ILP because it is the default process; permission to use one of the other processes is subject to FERC approval. The ILP has two main parts, the pre-filing process and the post-filing process. The regulations defining the process can be found in the Code of Federal Regulations Title 18 Part 5 on the website: ecfr.gpoaccess.gov. 


\subsubsection{Pre-Filing Process}

The pre-filing process officially begins when the Notice of Intent (NOI) and Pre-Application Document (PAD) are filed. After FERC receives them, it begins a review followed by a public comment period. The comment period allows public stakeholders to file their thoughts on the proposed project. At the conclusion of the comment period, FERC will decide which process will be used and the post-filing process will begin.

In the NOI the licensee should state its intent to file for an original license. It should also include the name and address of the licensee, the type, location, and installed capacity of the project, as well as a list of names and addresses of all local political subdivisions affected by the project (see 18 CFR 5.5 (b)(8)).

The PAD "makes known all existing engineering, economic, and environmental information relevant to licensing the project that is reasonably available, or can reasonably be obtained with due diligence" as well as conveying the applicant's schedule for completing and filing the application. Specific information on what information should be in the PAD can be found in 18 CFR 5.6. For the ILP, the PAD must include a proposed date and location for the scoping meeting as well. The purpose of the meeting is to initiate scoping and to review and discuss conditions, objectives, and information in order to finalize the pre-filing process plan and schedule.

Prior to filing the application, the applicant is required to consult with relevant agencies and stakeholders regarding project design, impact, reasonable alternatives, and required studies. A list of agencies to be contacted may be requested from the Director of the Office of Energy Projects (see 18 CFR 5.1 (d)).

Sixty days after FERC receives the NOI and PAD, it will issue a notice of commencement (proceeding). The notice will include in it the initiation by FERC of informal consultations under the Endangered Species Act (ESA), Magnuson-Stevens Fishery Conservation and Management Act, and the National Historic Preservation Act (NHPA). These are all defined in the section below: "Necessary Federal and State Regulations."

The notice also includes the date and place of the public scoping meeting and project site visit which will be held within 30 days of the notice. FERC will also publish Scoping Document 1 (SD1) at the same time as the notice. The purpose of this document is to identify the scope of issues to be addressed in the National Environmental Policy Act (NEPA) analysis, the level of analysis required, waterway plans that would be considered in the analysis, and a process plan and schedule. This is the beginning of the NEPA scoping process, which occurs concurrently with the pre-filing consultation. In addition to the consultations named above, the scoping process should include the consultations for the laws and regulations listed in the "Necessary Federal and State Regulations" section that follows.

Sixty days after the notice is issued all involved agencies and tribes must have provided FERC with comments on the PAD and SD1, including information and studies needed. If necessary 45 days later Scoping Document 2 (SD2) should be issued addressing the comments to SD1. 
Based on the comments and study requests, the applicant must prepare a proposed study plan to be filed 45 days after the end of the comment period. The study plan should include time for study plan meetings (held within 30 days of the deadline to file the study plan) to discuss and resolve comments and outstanding issues. Stakeholders have 90 days from the time the study plan is filed to comment and work with the applicant to resolve any disputes. After the close of the comment period, the applicant has 30 days to create a revised study plan followed by a shorter 15 day comment period. After this second iteration the Director of the Office of Energy Projects will make a study plan determination.

Agencies and tribes that have authority to provide mandatory conditions based on the Federal Power Act or Clean Water Act can dispute the study plan determination within 20 days. FERC then has 20 days to assemble a panel to resolve the dispute and the applicant has 25 days to file any comments or information regarding the dispute. The panel has 50 days from the notice of study dispute to deliver findings and recommendations to the Director of the Office of Energy Projects, who then has 20 days to issue a written determination.

After a final study plan is approved the applicant should begin with the studies identified. Within one year the applicant must file an initial progress report on the studies conducted. It is possible (but difficult) to obtain approval to modify the study plan based on the results of the initial progress report and study data.

The FERC license application should be filed by the applicant once the studies are nearing completion. The application content depends on the licensing process (TLP, ALP, or ILP) and type of project (whether it has already been built, is yet to be built or is to be modified, and how large of a capacity will be built). In general the application contains general information, an initial statement, and specified exhibits. The specified exhibits required can be found in 18 CFR 5.18, 4.32(a), 4.38(f), 4.41, 4.51,4.61, 16.10, and 16.11.

\subsubsection{Post-Filing Process}

After receiving the application, FERC will issue a public tendering notice that contains a preliminary schedule for processing the application. FERC will then decide to accept or reject the application. If there are deficiencies, the applicant will be notified and given time for correction (no more than 90 days).

Once the application is accepted, FERC will issue the Notice of Ready for Environmental Analysis (REA); then a 60 day period for public comment begins and FERC has 45 days (after the first 60) to reply to the comments.

Sixty days after the notice of REA, the applicant must file for Water Quality Certification with the Oregon Department of Environmental Quality (DEQ) and submit proof of request for certification. DEQ assesses the project to ensure that construction, operation, maintenance, and decommissioning of the project will not violate state or federal water quality standards or become a future source of pollution. The DEQ may grant, conditionally grant, or deny certification. DEQ has one year from the time the application is received to reach a decision. 
FERC has 180 days to issue the NEPA document, the draft Environmental Assessment (EA) or draft Environmental Impact Statement (EIS). Any comments on the draft EA or EIS must be filed in the time specified in the draft EA or draft EIS notice (30 to 60 days). After the comment period ends and any issues are resolved, the Final EA will be prepared. Finally, the license order (including the terms and conditions for project operation) is issued.

\subsection{FEDERAL AND STATE REGULATIONS}

\subsubsection{Federal Regulations:}

The NEPA Analysis ensures that federal agencies evaluate potential environmental impacts of the project. This includes effects on natural resources, the natural and human environment, and human uses. The NEPA document should include all the information required to satisfy all necessary federal and state consultations.

As part of the ESA Consultation the Services (National Marine Fisheries Service (NMFS) and U.S. Fish and Wildlife Services (USFWS)) ensure that the project does not jeopardize or result in destruction or adverse impacts to threatened or endangered species (including species habitat). If both FERC and the Services agree that the project will not likely have adverse impacts, the consultation is concluded. Otherwise, a formal consultation must begin.

The Magnuson-Stevens Act ensures that Essential Fish Habitat is protected. NMFS must be consulted on impacts to essential fish habitat, such as changes in temperature, nutrients or salinity of both the water column and underlying surface, and conservation measures. NMFS will provide Conservation Recommendations at the conclusion of the assessment. In the NHPA $\S 106$ Consultation agencies (including FERC) are required to identify and assess the effects of a project on historic resources as well as give the Advisory Council on Historic Preservation an opportunity to comment on the project. They must also consult with all other effected state and tribal offices. There are three stages of consultation: initiation of consultation, assessment of adverse effects, and resolution of adverse effects. All are expected to take 30-60 days to complete.

Migratory Bird Treaty Act Consultation: USFWS encourages applicants to account for migratory bird impacts including collision avoidance, minimization, enhancement, monitoring, and adaptive management for the protection of migratory birds. There is no formal timeline.

Fish and Wildlife Coordination Act Consultation: All federal agencies (including FERC) must consult with the Services and state agencies regarding fish and wildlife. This ensures that construction, maintenance and operation of the project prevent the loss of or damage to fish and wildlife resources.

Marine Mammal Protection Act: NMFS may authorize the project to "harass" small numbers of marine mammals incidentally but not intentionally provided it will not negatively impact the species (Incidental Harassment Authorization, IHA). An IHA requires monitoring and reporting. If there is potential for serious injury or a "take" a Letter of Authorization is needed and will require much more time (up to 2 years). Take is defined as harassing, hunting, capturing, or killing any marine mammal or attempting to do so. Harassment is 
defined as pursuit, torment or annoyance that has the potential to injure a marine mammal or disrupt its behavior.

River \& Harbors Act $\$ 10$ Review: The Army Corps of Engineers (COE) must authorize any structures or activities obstructing or altering navigable waters. If there are any threatened or endangered species in the area, NMFS and FWS must be consulted by COE before a decision is made. Also, opportunity for public hearings is required.

Clean Water Act $\$ 404$ Review: COE must authorize dredge and fill activities to conserve and restore waterways. An inter-agency consultation with federal and state agencies will take place. If there are any threatened or endangered species in the area, NMFS and FWS must be consulted by COE before a decision is made. Also, opportunity for public hearings is required.

USCG Review: A Private Aids to Navigation Permit needs to be obtained from the USCG. This is authorization for the owner/operator to properly mark the structure per U.S. navigation standards. COE must approve the $\S 404$ and $\S 10$ permits before this process begins.

\subsubsection{State Regulations:}

In this case, the proposed project would be developed in the state of Oregon, so that state is the focus of this section.

Coastal Zone Management Act (CZMA) Consistency Review: The Oregon Department of Land Conservation and Development reviews federal activities for a consistency determination, issuing either a concurrence or an objection. Federal actions must be consistent with the Oregon Coastal Management Plan.

Oregon State Removal-Fill Review: The Department of State Lands (DSL) must issue a permit to remove, alter, or fill materials in state waters (likely needed for anchoring and cables). It authorizes short term use and may specify the conditions of use. It usually takes 3-4 months to process.

Oregon State Ocean Shores Review: The Oregon Parks and Recreation Department must authorize a structure to be made or removed on or under the ocean shore (such as maintenance buildings and cables).

Oregon State Special Uses Lease: A Special Uses Lease from the DSL authorizes a use of state-owned land that is not specifically governed by other DSL rules. This will likely be the case for the transmission cable that passes through Oregon state waters. The application must be submitted 180 days before installation.

\section{PROCEDURAL CONSIDERATIONS}

At the time of this writing, there is no specialized regulatory path that has been defined for the development of hybrid wind/wave energy systems in federal waters. Indeed, while the agencies overseeing the development of these resources are willing partners, and reportedly working together to develop a more specialized process, the current prescribed 
path is confusing, convoluted and intimidating. In essence, the process as it stands is "one atop the other".

Any changes that occur to the current set of regulations will be subject to process that, one way or the other, will allow for the public to contribute to the discussion. In the case of prospective changes to the jurisdictions of the agencies, an unlikely outcome, legislated changes would be required. For changes in the way the agencies' existing authorities are administered a rulemaking would be required; this has public comment periods built into the process. A fuller discussion of the potential for, and process required, to create a specialized regulatory system for hybrid wind/wave energy devices is beyond the scope of this paper.

\subsection{ISSUES THAT ARISE}

While the intentions of the MOU and the guidance document are quite clearly to be helpful, there is still a considerable amount of work required to bring clarity to the hybrid systems regulatory picture. Some discussion is provided below.

Further clarity is needed with respect to the issues and policies for which each agency is/will be responsible. Which issues are considered in BOEMRE's leasing decision-making process? And how does this differ from the review FERC will conduct as part of the licensing process? For example, which of the agencies will be responsible for reviewing the potential effects of a submarine cable that extends from the device to the shore? If each will be reviewing the same information at slightly different times it presents an opportunity to eliminate redundancy.

FERC's apparent deference to the BOEMRE process (i.e. FERC not providing authorization until BOEMRE has provided authorization) has the potential to build years into the overall process. The developer would prefer to complete one data set and submit it to a single agency at one time. And, if they become concurrent, how will they be coordinated?

Moreover, since the Department of Interior and FERC are the only parties to the MOU, it remains to be seen what role will be afforded to the National Oceanic and Atmospheric Administration (NOAA), the nation's premier oceans stewardship agency, which possesses the most relevant knowledge and expertise concerning protection of our marine ecosystems.

Section III of the MOU addresses the handling of hybrid (wind/hydrokinetic) projects and projects that straddle the boundaries between state waters and the OCS very lightly. The agreement states the intent "...to work together to the extent practicable to develop policies and regulations" for such projects. This is encouraging, but does not set forth the specific guidance that industry or environmental advocates seek in furthering their efforts to promote sustainable renewable energy.

Finally, a developer that proposes a project that incorporates both wind and wave energy will be required to fulfil the requirements of BOEMRE's SAP and COP, in addition to those embedded in one of FERC's three licensing processes. This raises the possibility that the developer will be required to work through three separate NEPA processes for the same 
body of water over the course of several years. While it is clear that each step could inform the subsequent ones, this is a timely and expensive proposition.

\section{CONCLUSION}

At the time of this writing, and since the first quarter of 2011, representatives from both FERC and BOEMRE have indicated that work is ongoing with regards to establishing a permitting system appropriate for hybrid wind/wave technologies like the WindWaveFloat. This is encouraging and important work. The present state of play for a developer proposing to develop a project using such a system is confusing and intimidating. Perhaps more importantly, the present system includes enough unknowns to introduce more risk to a project, at a time when technologies are still evolving.

At the same time, no commercial projects have yet been proposed, and the technologies are still relatively immature. Hopefully, in this case, the regulatory environment for these important technologies will be developed in such a way as to encourage commercial activity, so that the promise inherent in them will benefit the citizens of the United States and other places around the world. 


\section{APPENDICES}

\section{APPENDIX " $A$ ”}

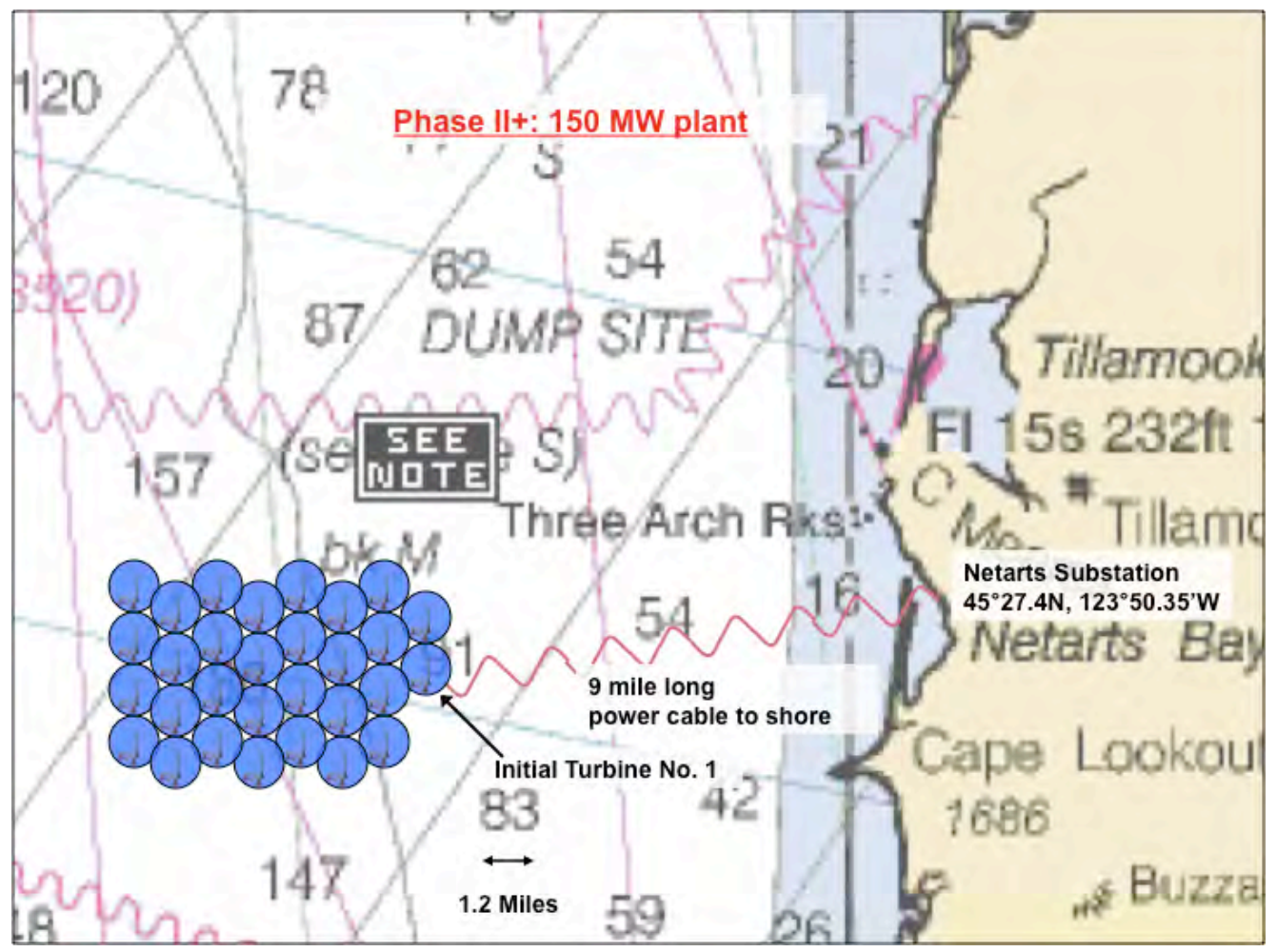

Figure 5: Location /lavout of Case Proiect

\section{APPENDIX “B”}
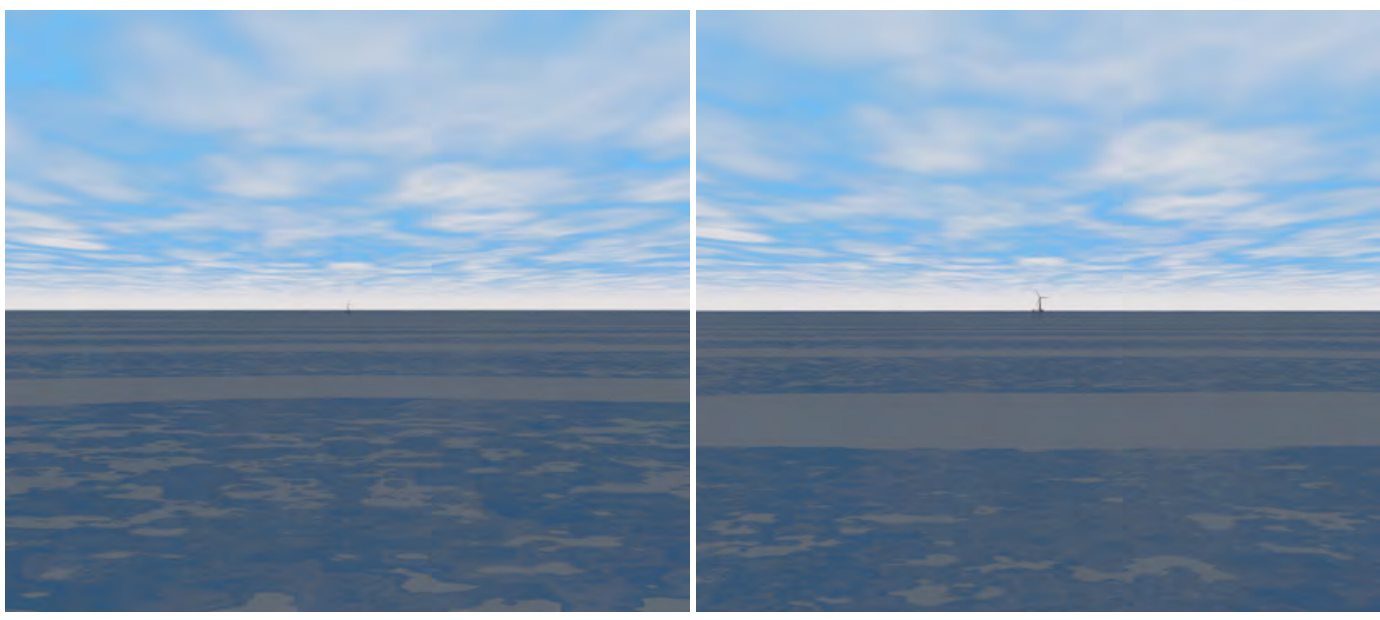

Figure 6: Views from the beach 


\section{REFERENCES}

i. $\quad$ European Strategic Energy Technology Plan (SET-Plan) COM (2007) 723 Final EC communication to European Parliament

ii. (2009) The WWF website. [Online] Available: www.panda.org/about_wwf/what_we_do/climate_change/problems/impacts/i ndex.cfm

iii. N. Stern, "The Stern Review of Economics of Climate Change" - HM Treasury, Oct. 2006.

iv. Isaacs and Seymour, "The Ocean as a Power Resource," International Journal of Environmental Studies vol 4(3), pp201-205, 1973.

v. Delivering offshore wind power in Europe, EWEA, Dec 2007

vi. T.Pontes, "Assessing the European Wave Energy Resource," Journal of Offshore Mechanics \& Artic Engineering, vol. 120, pp223-26. 1998.

vii. vii "Technology White Paper on Wave Energy Potential on the U.S. Outer Continental Shelf." Minerals Management Service, Renewable Energy and Alternate Use Program, U.S. Department of the Interior. May 2006.

viii. viii Energy Information Administration, Forecasts and Analyses.

ix. (2011) The Statoil website. [Online]. Available: http://www.statoil.com/en/TechnologyInnovation/NewEnergy/RenewablePo we rProduction/Onshore/Pages/Karmoy.aspx

x. D. Roddier, C. Cermelli, A. Weinstein "WindFloat: A Floating Foundation For Offshore Wind Turbine. - Part I: Design Basis and Qualification Process " in OMAE 2009, 2009, paper.

xi. Bedard, R., et al., 2005, Final Summary Report, Project Definition Study, Offshore Wave Power Feasibility Demonstration Project, EPRI Global WP 009 - US Rev 1, Jan. 14, 2005.

xii. $\quad$ xii Burton T. et al. "Wind Energy Handbook", Wiley, 2001

xiii. $\quad$ xiii Cape Wind MEPA Certificate on the Final Environmental Impact Report, 2007

xiv. $\quad$ xiv Talisman Energy, "Beatrice Wind Farm Demonstrator Project Scoping Document", 2007

xv. $\quad$ xv (2011) The FERC website. [Online] Available: http://www.ferc.gov/legal/majord-reg/mou/mou-doi.pdf

xvi. $\quad$ xvi (2011) The FERC website. [Online] Available:

http://www.ferc.gov/industries/hydropower/indusact/hydrokinetics/pdf/mms080309.pdf

xvii. $\quad$ xvii Renewable Energy and Alternate Uses on the OCS. MMS-AWEA: Project Timelines. January 2010

xviii. xviii (2011) The DOI website. [Online] Available: http://www.doi.gov/news/pressreleases/Salazar-Launches-Smart-from-theStart-Initiative-to-Speed-Offshore-Wind-Energy-Development-off-the-AtlanticCoast.cfm

xix. $\quad$ xix (2011) The FERC website. [Online] Available: http://www.FERC.gov 
Appendix E: WindWaveFloat: Combining offshore wind turbines and WEC in a single structure 


\title{
WindWaveFloat: Combining offshore wind turbines and WEC in a single structure
}

\author{
Kevin S. Banister ${ }^{1}$, Alla Weinstein ${ }^{2}$, Dominique Roddier ${ }^{3}$ \\ "Principle Power, Inc. \\ Seattle, WA and Berkeley CA, United States of America \\ ${ }^{1}$ kbanister@principlepowerinc.com \\ ${ }^{2}$ aweinstein@principlepowerinc.com \\ ${ }^{3}$ droddier@principlepowerinc.com
}

\begin{abstract}
This paper summarizes the theory behind the modeling that was performed to incorporate three different Wave energy Converters (WEC) into the WindFloat hull. The WindFloat is a 3legged floating structure supporting a very large (>5MW) wind turbine. The three columns form an equilateral; heave plates are fitted at the base of the column, increasing the added mass of the total structure, minimizing pitch and heave motions. The structure is moored to the seabed, and an electrical cable connects the turbine to the shore. By adding a WEC to the structure, one can improve the overall economic s of the project. The cost of the WEC alone needs to be less than the energy it produces. However, by sharing both mooring and power infrastructure, the WEC economics are greatly advantageous over a farm of WEC alone. The first WEC investigated is a cylindrical oscillating water column fitted around the columns of the WindFloat not supporting the wind turbine. The second WEC investigated is a single point energy absorber, fitted inside the WindFloat and connected horizontally to the columns. The third is an oscillatory plate, fitted between columns. The paper also explores the regulatory environment for these systems in the US.
\end{abstract}

Keywords - ocean renewable energy, wave energy conversion, offshore wind, floating support structure, hybrid renewable energy systems

\section{INTRODUCTION}

The WindWaveFloat represents a technological solution for harvesting energy from offshore renewable resources wind, wave and tidal. It overcomes conflicting demands for stable and constrained support structure motion for wind capture, and hydrodynamic free motion for ocean energy capture, providing a floating, offshore multi-purpose renewable energy production support structure able to host wind/ocean energy converters.

The environmental and ecological impacts of "Green House Gases" (GHG) and global warming are now universally acknowledged, as are their economic implications (e.g. [1],[2],[3]). The world's population currently consumes 15 TW of power that is predicted to increase to 30 TW by 2050 . There is therefore a pressing need to meet the world's current and future energy demands from renewable and non-GHG emitting sources. Ocean energy resources are vast. Globally, the estimate of deep-water wave energy potential is in the range from $1 \mathrm{TW}$ to $10 \mathrm{TW}$ [4]. This, coupled with similar energy potential of deep water offshore wind resources, presents a compelling proposition.

Early development of large-scale offshore renewables will play an important role in achieving global goals to reduce GHG emissions and the use of depleting conventional energy sources. Seas and oceans cover over $70 \%$ of the earth's surface, offering huge potential for renewable energy from wind[5], wave[6], tidal, thermal, and osmotic sources.

\section{A. Business Drivers}

WindWaveFloat, along with other innovations in the field of multi-national interlinked offshore-grids, osmotic power projects and offshore energy storage [7] are needed to make a meaningful impact in the energy space. Ocean energy offers the benefits of significant resources without competing for land use, but suffers from projected high operating and installation costs. The WindWaveFloat concept offers a potential ability to increase the energy production from each floating support structure by approximately 30\%. For example, wave energy converters installed in a WindFloat, a floating support structure for large offshore wind turbines, could potentially increase each unit's nameplate capacity from $5 \mathrm{MW}$ to $7.5 \mathrm{MW}$ without a need for additional cabling, space or permits. The ultimate result could lead to the reduction of levelized energy costs by approximately $25 \%$, increase in the overall capacity factor by approximately $10 \%$ as compared to the wind only generation, and increased stability of the electrical power delivered to the grid.

This paper will report on preliminary results achieved through investigations integrating an oscillating water column system, a novel point absorber system and oscillating plates into the WindFloat design. The primary purpose of these studies was to validate models designed to understand overall performance of the integrated system, including numerical tools and scaled model tank testing. In each case, a model test campaign at $1 / 78.5$ scale was conducted at the UC Berkeley ship model testing facility, which features a $200 \mathrm{ft}$ long wave flume. This university lab is well suited for research and development and many other wave energy devices have been tested there in the past. 
The commercial, environmental and policy consequences of successfully integrating the conversion of wave and wind energy resources into a single device are large. But they will also be contingent on several factors that will follow on from the discoveries initiated and discussed in this paper. These factors will include constructability at scale, logistics and commodity supply and issues regarding finance and insurance. In addition, and not the least of these follow-on issues, will be the retention of favourable policies intended to bolster the renewable energy industry and the execution of laws intended to regulate the industry. Each of these factors is serious, but because current regulatory schemes largely do not contemplate hybrid energy systems, this paper will also explore regulatory considerations for this type of innovative energy capture system in US waters.

\section{OFFSHORE WIND AND THE WINDFLOAT}

The market for offshore wind has enjoyed exponential growth over the past 10 years primarily in the North Sea, UK, Netherlands and lower Scandinavia. Technological and economic limitations have imposed water depth limitations on offshore wind development to date. The emergence of floating support structures serves to eliminate the current limitation on water depth for offshore wind development. The market implications are significant as depth insensitivity opens up the sites that were previously unattainable in the United States and Europe for development.

Several floating support structures have been publically announced. All are at various stages of development. Statoil's HyWind project, a full-scale demonstration featuring a Siemens 2.3 MW offshore wind turbine has been deployed off the coast of Norway since 2009[8]. Principle Power's demonstration WindFloat is planned to be deployed off the coast of Portugal in the third quarter of 2011; this will feature a Vestas $2 \mathrm{MW}$ offshore wind turbine. A common theme exists throughout these concepts; a reliance on technology and development methodology from the Oil and Gas industry.

The WindFloat is intended to allow integration with any commercially available, horizontal axis offshore wind turbine; other floating support structures may rely on custom turbine designs.

\section{A. The WindFloat}

WindFloat[9] is a semi-submersible structure, which uses a combination of static ballast, heave plates and asymmetric design to achieve excellent dynamic stability performance. The performance is such that commercially available turbines can be used following integration work on the part of the wind turbine manufacturer and Principle Power. A catenary mooring is employed to further decrease complexity and economic impact on the system. Structural efficiency is maximized by locating the large turbine payload atop a load bearing column. Mean wind induced thrust forces on the system are mitigated by a secondary closed loop active ballast system. Assembly and qualification of the system is completed quayside in a controlled environment. Offshore operations are all but eliminated as the WindFloat can be towed to and from port for installation or maintenance.

The WindFloat (Figure 1) consists of a column-stabilized floating support structure with water-entrapment plates and an asymmetric mooring system. A wind turbine mast is positioned directly above one of the stabilizing columns.

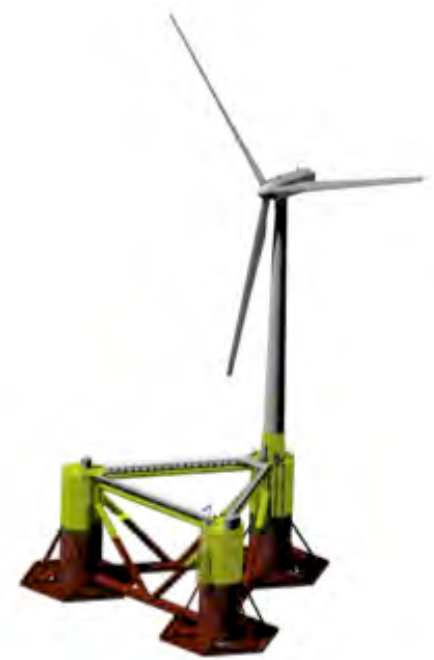

Fig 1 WindFloat hull and Turbine

The diameter at the base of the turbine tower is close to the column diameter in order to maximize continuity of the structure, leading to minimized stress concentration in a critical area of the structure where bending moments are the highest (due to wind-induced overturning moment) and large tubulars connect to the other stabilizing columns. Two other stabilizing columns are spread out as to form an equilateral triangle between the 3 column centers. A boat landing is installed on one or both of these columns to access the structure. The columns are interconnected with a truss structure composed of main beams connecting columns and bracings connecting main beams to columns or other main beams. The general dimensions are shown in Table 1.

TABLE 1

WiNDWAVEFLOAT GENERAL DIMENSIONS

\begin{tabular}{ll}
\hline \multicolumn{1}{c}{$\begin{array}{c}\text { WindWaveFloat General Dimensions } \\
\text { for the NREL 5 MW turbine }\end{array}$} \\
\hline Column diameter & $10 \mathrm{~m}$ \\
Length of water entrapment plate edge & $15 \mathrm{~m}$ \\
Column center to center & $46 \mathrm{~m}$ \\
Pontoon diameter & $2.1 \mathrm{~m}$ \\
Operating draft & $17 \mathrm{~m}$ \\
Airgap & $10 \mathrm{~m}$ \\
Bracing diameter & $1.5 \mathrm{~m}$ \\
DISPLACEMENT & 4832 tonnes \\
\hline
\end{tabular}

A horizontal water-entrapment plate is located at the base of each column. Stiffeners cantilevered from the bottom of the columns with bracing tying these stiffeners back to the columns support the plates. The water-entrapment plates provide additional hydrodynamic inertia to the structure due to 
the large amount of water displaced as the support structure moves. In addition, vortices generated at the edge of the plates generate large damping forces that further impede the support structure motion.

\section{WAVE ENERGY CONVERSION AND INTEGRATION}

Deep-water (more than $50 \mathrm{~m}$ ) wave energy resources are known to be $30 \mathrm{~kW} / \mathrm{m}$ and greater [10], offering vast potential for power generation. Offshore wave energy conversion mechanisms exploit the powerful wave regimes available in deep water and are much less dependent on the geomorphological conditions of the coastline.

There are many wave energy conversion systems in development. The main principles of wave energy conversion are based on oscillating bodies, oscillating water columns, overtopping devices, and lift-induced rotation. An important part of most power take-off (PTO) mechanisms is to have a stable fixed reference for the production of useful work. The energy is extracted through the relative forces and motions between the moving body and the fixed base (sea bottom, pile, floating frame, etc.) to which it is connected.

\section{A. The WindWaveFloat and Study Considerations}

The WindWaveFloat design is based on the PTO solutions that are deployed and directly mounted in and/or onto the WindFloat. Using the WindFloat support structure as the basis for a new, integrated wind/wave energy conversion device for deep ocean offers many advantages as well as challenges. The use of the floating wind support structure as the reference frame for wave PTOs may result in larger motions due to the forces resulting from energy extraction, unless a proper control procedure is introduced. This control may be needed to balance the requirement of floating support structure stability against energy production. The final design for the integrated WWF needs to be optimized to enable the most efficient and cost effective power production between the wind turbine and the wave energy PTOs.

The WindWaveFloat is positioned with a catenary mooring, which consists of 4 mooring lines, two on column 1, which carries the turbine, and one on each other column. Each line is made of 3 sections: 3 -inch chain at the fairlead, 5-inch polyester, and 3 -inch chain to the anchor at the bottom. A clump weight is placed between the upper chain section and the polyester rope to control the tension. The pretension on the mooring lines is $535 \mathrm{kN}$. The displacement of the support structure is 4832 metric tons.

Two numerical models of the base case are developed for use with the different types of wave energy device.

In WAMIT, a frequency domain diffraction-radiation program, the behavior of the support structure in incident waves is determined based on linear theory. A high order representation of the geometry is used. The water entrapment plates are thin plates represented with dipoles. The submerged columns and main beams are modeled up to the waterline. The mass matrix is based on the support structure mass properties as defined by the WindWaveFloat. An equivalent damping and stiffness matrix are used to model respectively the effect of viscous damping on the water entrapment plates and the effect of mooring. These matrices are adjusted based on the OrcaFlex model described below, and have been validated by multiple model tests.

A model of the WindFloat is also generated in OrcaFlex, a time-domain solver for the 6-degree of freedom equations. The response of the system to wave dynamics is obtained from WAMIT. Dynamic coefficients, such as added mass, damping and hydrostatic stiffness are transferred from the WAMIT output files, as well as diffraction forces and quadratic drift coefficients. Non-linear forces are added to the 6 degree of freedom equations as needed. Mooring components are modeled in 3D. The effect of viscosity on the columns and water entrapment plates is represented with Morison's formulas.

1) Oscillating Water Column: In an oscillating water column (OWC) water enters into a chamber that contains air. As waves pass, the amount of water in the chamber rises and falls like a piston, thereby sequentially compressing and decompressing the air. These positive and negatives changes in pressure result in the ability to direct the air through a bidirectional turbine coupled to an electric generator. A Wells turbine is a common choice for this type of application.

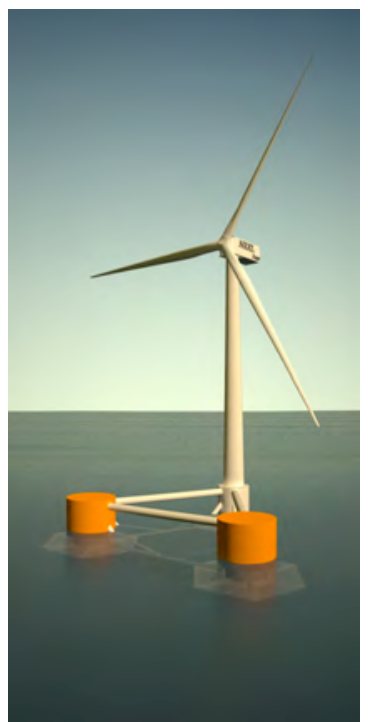

Fig 2 WindWaveFloat featuring Oscillating Water Column Systems.

In this case, preserving the stability performance of the original WindFloat design is deemed to be a priority, so the chambers are built around the columns that are not responsible for supporting the wind tower and turbine, and for design simplicity's sake extend only 240 degrees around the column, avoiding truss-connecting points on the column. The effect of the OWC system on support structure motions needs to be understood for both how it might affect wind turbine performance and how it might affect the performance of the wave energy device. 
The WindWaveFloat floating support structure used in all these analyses is identical to that designed to support a $5 \mathrm{MW}$ NREL wind turbine (figure 2). The Wells turbine modelled is assumed to have a turbine radius of one meter. The optimal design parameters defining the size, and ultimately the precise placement, of the turbine should be the subject of further study.

2) Spherical Point Absorber: In this section, the integration of a single point absorber is investigated. The SWEDE (Spherical Wave Energy DEvice) is a spherical point absorber situated in the centre of the structure and attached to the WindFloat columns by three springs and dampening elements (see figure 3). A point absorber is a floating system that absorbs energy in all directions through its movements on the waters surface. A point absorber is usually deigned to resonate so that its harnessed power is maximized. A spherical floater was selected because it responds well to heave with very little pitch motion.

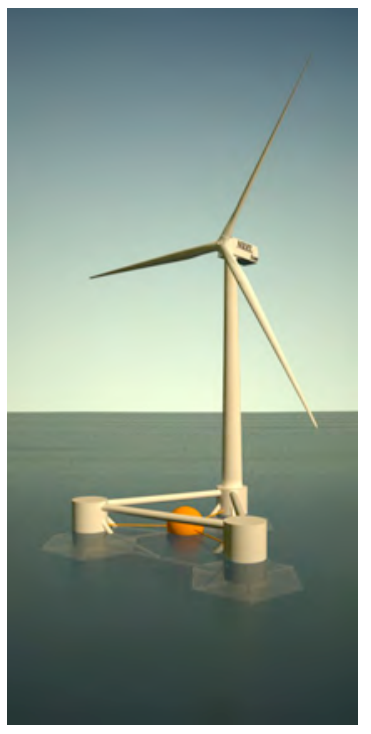

Fig 3 WindWaveFloat featuring SWEDE.

The single device SWEDE is a spherical floater installed in the center of the WindFloat support structure. The floater is attached to the column of the WindFloat by using three lines (see figure 4) representing the power take-off system, the precise nature of which is unknown at this stage.

The lines are modeled as combined spring and independent damper units. The spring can take both compression and tension, and has a linear length-force relationship. The damper velocity-force relationship is also linear. The line characteristics are determined using 100-year storm conditions to assure that the floater never hits the WindFloat columns or beams, and so that the tensions in the lines stay within their design values.

Hydrodynamic loads on the floaters are calculated using Morison's equation. Added mass and drag forces are applied only to those parts of the support structure that are in the water at the time for which the force is calculated. The six degree-of-freedom added-mass coefficients, damping coefficients, and wave exciting loads of the floater are obtained with WAMIT using a single body analysis. Hydrodynamic coupling is neglected.

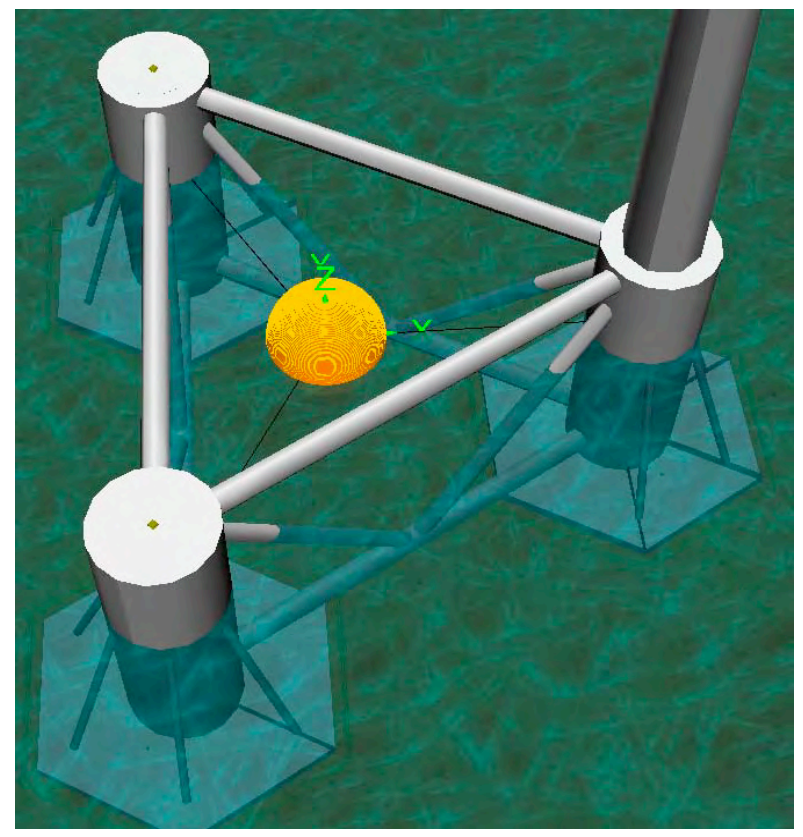

Fig 4 Lines connecting SWEDE to columns represent the power take off system.

3) Oscillating Wave Surge Convertor: The Oscillating Wave Surge Convertor (OWSC) comprises a near-surface collecting system mounted on a pivoting arm installed on the seabed. The arm oscillates like an inverted pendulum in response to the surrounding waves. In this case the plates are mounted on the structure main beams, outside of the water (see figure 5). This has the significant advantage of having the possibility of being completely removed from any wave actions during large storms, as the beams are designed to be dry and the flaps should be able to be locked in a horizontal position.

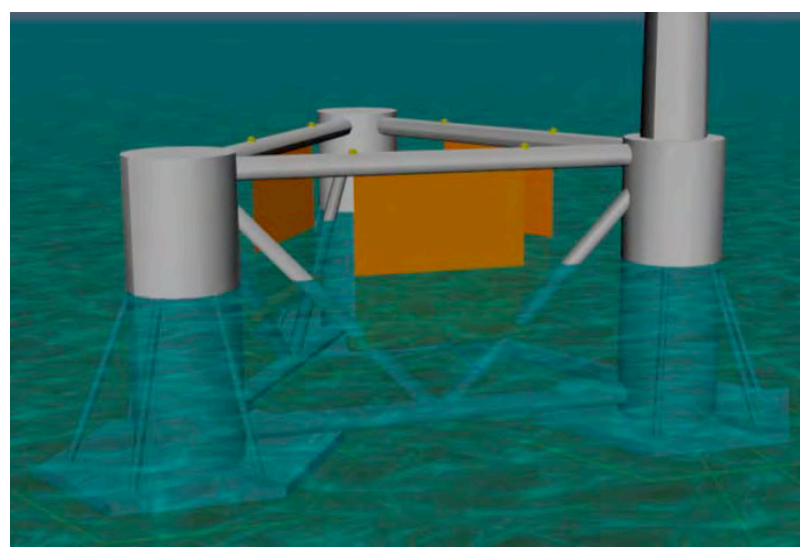

Fig 5 The rigid flaps of the OWSC

The Oscillating Plates concept consists of three rectangular flaps (flat stiffened vertical plates) hinged on the 
three top main beams of the WindFloat support structure (see figure 6). The flaps oscillate back and forth as the waves hit them. The lower edge of each flap is attached to two lines representing the power take-off system (unknown at this stage but either in the hydraulic or electrical motor category) mounted on the WindFloat support structure. The hinge mechanism is modeled by connecting the flaps to line members attached to the WindFloat support structure, with an infinite bending stiffness at the flap-line connection in all directions. Similarly, the six lines are modeled as combined spring and independent damper units. The spring can take both compression and tension, and has a linear length-force relationship. The damper velocity force relationship is also linear. The line characteristics are determined using 100-year storm conditions and therefore assuring that the flap maximum angle will stay within its design range.

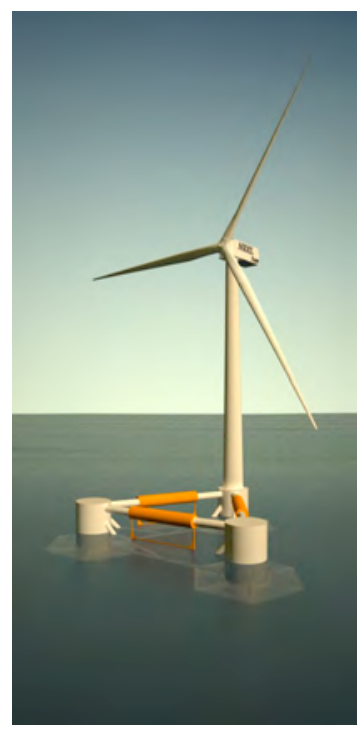

Fig 6 WindWaveFloat featurig the oscillating wave surge converter.

Similarly to the other WWF concepts, hydrodynamic loads on the flaps are calculated using Morison's equation. The six degree-of-freedom added-mass coefficients, damping coefficients, and wave-exciting loads of the flaps are obtained with WAMIT dipole elements. The flaps are modeled in OrcaFlex by using rectangular vessels instead of 6DOF floaters.

\section{REGULATORY CONSIDERATIONS IN THE US}

While this paper is primarily focused on the technical issues associated with the integration of wave energy conversion devices into the WindFloat floating support structure for offshore wind, regulatory considerations play an important role in determining the economic viability of these hybrid renewable energy systems.

In order to make advancements in marine renewable energy technology, devices need to be tested and the effects of their interaction with the environment need to be assessed. Substantive progress in this regard can only be achieved when full scale devices have been placed, commissioned and are operating in the locations where they will be deployed at commercial scale.

\section{A. Regulatory Authority}

To date, there has been no attempt to license (receive public authorizations to construct and operate a project) a hybrid device in the United States. In the US, the current jurisdictions give the Federal Energy Regulatory Commission (FERC) authority to grant a license for the wave energy component of the system. The Department of Interior's Bureau of Ocean Energy Management, Regulation and Enforcement (BOEMRE) has the authority to lease the land on the outer continental shelf, or OCS (i.e. beyond 3 miles from shore), for the entire footprint and to permit construction and operation of the wind portion of the project.

The two agencies have recognized the challenge to developers multiple authorizing bodies represent. This recognition suggests the need to develop a single process, which will achieve greater efficiencies in both time and money. To that end, DOI and FERC entered a memorandum of understanding (MOU) dated April 9, 2009 which states: "[DOI and FERC] agree to work together to the extent practicable to develop policies and regulations with respect to OCS hydrokinetic projects to carry out the purpose of this MOU. This will include, among others, processes to address hybrid (wind/hydrokinetic) projects and projects that straddle the boundaries between state waters and the OCS."[11]

For any proposed changes to the regulatory process to take effect however, will require either legislative change (which is unlikely) or a rulemaking (which will take months to complete). Neither has been initiated at this time. In the meantime, the two federal agencies have different (and time sensitive) processes for approving a project.

1) Bureau of Ocean Energy Management Regulation and Enforcement: BOEMRE issues leases and grants for renewable energy projects in the OCS. There are two types of leases and two types of grants: commercial lease, limited lease, Rights-of-Way grant, and Rights-of-Use and Easement grant. BOEMRE is required to issue all of the above competitively unless it is determined there is no competitive interest.

2) Federal Energy Regulatory Commission: There are three possible processes for obtaining a hydrokinetic license from the Federal Energy Regulatory Commission. The three possible processes are the Integrated Licensing Process (ILP), Traditional Licensing Process (TLP), and Alternative Licensing Process (ALP). The ILP is the default process used and both the TLP and ALP require pre-approval from FERC. The following detail is for the ILP because this is the default process and it is unknown whether the Commission will approve a request from Principle Power to use either the TLP or ALP. The ILP has two main parts, the pre-filing process and the post-filing process. 


\section{B. Federal Requirements}

FERC and BOEMRE are responsible for the overall regulation and leasing of offshore energy projects, but there is a myriad of other agencies that become fundamentally involved in the permitting process. Each of these is responsible for the protection of its own specific jurisdiction. Many of them are guided by the directives of the National Environmental Protection Act, or NEPA.

NEPA ensures that federal agencies evaluate potential environmental impacts of the project. This includes effects on natural resources, the human environment, and human uses. NEPA documentation will need to include all the information required to satisfy all necessary federal and state consultations.

As part of the Endangered Species Act Consultation the Services (National Marine Fisheries Service (NMFS) and U.S. Fish and Wildlife Services (USFWS)) ensure that the project does not jeopardize or result in destruction or adverse impacts to threatened or endangered species (including species habitat). If both FERC and the Services agree that the project will not likely have adverse impacts, the consultation is concluded. Otherwise, a formal consultation must begin.

1) The Magnuson-Stevens Act: The Act ensures that Essential Fish Habitat is protected. NMFS must be consulted on impacts to essential fish habitat, such as changes in temperature, nutrients or salinity of both the water column and underlying surface, and conservation measures. NMFS will provide Conservation Recommendations at the conclusion of the assessment.

2) The National Historic Preservation Act: In the NHPA $\S 106$ Consultation agencies (including FERC) are required to identify and assess the effects of a project on historic resources as well as give the Advisory Council on Historic Preservation an opportunity to comment on the project. They must also consult with all other effected state and tribal offices. There are three stages of consultation: initiation of consultation, assessment of adverse effects, and resolution of adverse effects. All are expected to take 30-60 days to complete.

3) Migratory Bird Treaty Act Consultation: USFWS encourages applicants to account for migratory bird impacts including collision avoidance, minimization, enhancement, monitoring, and adaptive management for the protection of migratory birds. There is no formal timeline.

4) Fish and Wildlife Coordination Act Consultation: All federal agencies (including FERC) must consult with the Services and state agencies regarding fish and wildlife. This ensures that construction, maintenance and operation of the project prevent the loss of or damage to fish and wildlife resources.

5) Marine Mammal Protection Act: The National Marine Fisheries Service may authorize the project to "harass" small numbers of marine mammals incidentally but not intentionally provided it will not negatively impact the species (Incidental Harassment Authorization, IHA). An IHA requires monitoring and reporting. If there is potential for serious injury or a "take" a Letter of Authorization is needed and will require much more time (up to 2 years). Take is defined as harassing, hunting, capturing, or killing any marine mammal or attempting to do so. Harassment is defined as pursuit, torment or annoyance that has the potential to injure a marine mammal or disrupt its behavior.

6) River \& Harbors Act $\$ 10$ Review: The Army Corps of Engineers (COE) must authorize any structures or activities obstructing or altering navigable waters. If there are any threatened or endangered species in the area, NMFS and FWS must be consulted by $\mathrm{COE}$ before a decision is made. Also, opportunity for public hearings is required.

7) Clean Water Act $\$ 404$ Review: COE must authorize dredge and fill activities to conserve and restore waterways. An inter-agency consultation with federal and state agencies will take place. If there are any threatened or endangered species in the area, NMFS and FWS must be consulted by COE before a decision is made. Also, opportunity for public hearings is required.

8) USCG Review: A Private Aids to Navigation Permit needs to be obtained from the USCG. This is authorization for the owner/operator to properly mark the structure per U.S. navigation standards. COE must approve the $\S 404$ and $\S 10$ permits before this process begins.

\section{State Requirements}

Each coastal state will have its own set of regulatory requirements dependent on the location and action proposed. For offshore activities, the state requirements will be more or less relevant depending on whether the proposed action is within state-controlled waters (generally within 3 miles in the US) or not. The following are representative requirements.

1) Coastal Zone Management Act (CZMA) Consistency Review: The State Department of Land Conservation and Development reviews federal activities for a consistency determination, issuing either a concurrence or an objection. Federal actions must be consistent with the Oregon Coastal Management Plan.

2) State Removal-Fill Review: The Department of State Lands (DSL) must issue a permit to remove, alter, or fill materials in state waters (likely needed for anchoring and cables). It authorizes short-term use and may specify the conditions of use. It usually takes 3-4 months to process.

3) State Ocean Shores Review: The State Parks and Recreation Department must authorize a structure to be made or removed on or under the ocean shore (such as maintenance buildings and cables)

4) State Special Uses Lease: A Special Uses Lease from the DSL authorizes a use of state-owned land that is not specifically governed by other DSL rules. This will likely be 
the case for the transmission cable that passes through state waters.

\section{STUDY CONCLUSIONS}

This paper provides a broad look at issues generated by the intent to develop a hybrid wind-wave energy device: the WindWaveFloat. It explains many of design steps taken to advance the technological concepts that will be required to integrate innovative power take off systems into the WindFloat concept.

At the same time, the paper recognizes that there are many other issues that will effect the delivery of renewable energy from promising hybrid systems like those begun to be described here. A focus was given to the regulatory environment in the US; subsequent work could focus on explicit recommendations to improve and accelerate that process.

The following sections provide conclusions on the technical elements of the study.

\section{A. Oscillating Water Column}

A model was developed to incorporate the specificities of the WindFloat support structure in the calculation of the power converted by the turbine. The effect of global support structure motion on the performance of the OWC is limited in the range of operational wave periods according to these preliminary results. The current numerical model neglects non-linear loads on the support structure, as well as turbine damping on the internal water free surface. Losses in the turbine rotor and generator are also neglected. Model tests and further analysis are needed to assess the importance of such losses.

The pneumatic efficiency of the turbine however was approximated with a modeled function of flow ratio which is meant to represent the Wells turbine. This model shows that turbine characteristics are essential in the smoothing and optimization of the power output. Different turbine parameters should be tested to optimize the design.

\section{B. Point Absorbers and Flaps}

The different modeled designs hardly affect the WindFloat support structure motion. Oscillating plates have the most significant impact by increasing the surge motion amplitude of the WindFloat support structure. No showstopper regarding the motion of the WindFloat support structure has been discovered during these numerical simulations. Structurally, the effect of the loading of the wave device on the WindFloat hull has yet to be investigated.

Initial predictions show that a maximum average power per wave amplitude squared of $150 \mathrm{~kW} / \mathrm{m} 2$ would be potentially harnessed. These performances occur with regular sinusoidal incoming waves at a zero-degree heading, and might decrease substantially with irregular incoming waves. These performances are most likely lower in reality, since shielding effects, radiation effects, and hydrodynamic interferences are not modeled here. Moreover, losses in the PTO systems are not taken into account. These values have not been validated by model tests and some empirical coefficients will need to be adjusted, hence the uncertainty in the absolute value is significant. However, the conclusions based on relative observations can be used to optimize the WEC devices.

It is premature to compare the devices at this stage, since the numerical models need to be validated by part-scale model tests. However, the spherical floater and the three oscillating plates seem to yield similar power performances.

\section{ACKNOWLEDGEMENT}

This work was funded in part by a DOE financial assistance agreement (DE-EE0002652).

\section{REFERENCES}

[1] European Strategic Energy Technology Plan (SET-Plan) COM (2007) 723 Final - EC communication to European Parliament

[2] (2009) The WWF website. [Online] Available: www.panda.org/about_wwf/what_we_do/climate_change/problems/imp acts/index.cfm

[3] N. Stern, "The Stern Review of Economics of Climate Change" - HM Treasury, Oct. 2006.

[4] Isaacs and Seymour, "The Ocean as a Power Resource," International Journal of Environmental Studies vol 4(3), pp201-205, 1973.

[5] Delivering offshore wind power in Europe, EWEA, Dec 2007

[6] T.Pontes, "Assessing the European Wave Energy Resource," Journal of Offshore Mechanics \& Artic Engineering, vol. 120, pp223-26. 1998.

[7] (2009) The Poseiden Energy website. [Online] Available: www.poseidonenergy.com

[8] (2011) The Statoil website. [Online]. Available: http://www.statoil.com/en/TechnologyInnovation/NewEnergy/Renewab lePowerProduction/Onshore/Pages/Karmoy.aspx

[9] D. Roddier, C. Cermelli, A. Weinstein "WindFloat: A Floating Foundation For Offshore Wind Turbine. - Part I: Design Basis and Qualification Process “ in OMAE 2009, 2009, paper.

[10] Bedard, R., et al., 2005, Final Summary Report, Project Definition Study, Offshore Wave Power Feasibility Demonstration Project, EPRI Global WP 009 - US Rev 1, Jan. 14, 2005.

[11] (2009) The FERC website. [Online] Availale: http://www.ferc.gov/legal/maj-ord-reg/mou/mou-doi.pdf 